University of Tennessee Health Science Center UTHSC Digital Commons

\title{
Enzyme Architecture and Flexibility Affect DNA Topoisomerase I Function
}

Mariè van der Merwe

University of Tennessee Health Science Center

Follow this and additional works at: https://dc.uthsc.edu/dissertations

Part of the Enzymes and Coenzymes Commons

\section{Recommended Citation}

van der Merwe, Mariè , "Enzyme Architecture and Flexibility Affect DNA Topoisomerase I Function" (2007). Theses and Dissertations (ETD). Paper 289. http://dx.doi.org/10.21007/etd.cghs.2007.0333.

This Dissertation is brought to you for free and open access by the College of Graduate Health Sciences at UTHSC Digital Commons. It has been accepted for inclusion in Theses and Dissertations (ETD) by an authorized administrator of UTHSC Digital Commons. For more information, please contact jwelch30@uthsc.edu. 


\title{
Enzyme Architecture and Flexibility Affect DNA Topoisomerase I Function
}

\begin{abstract}
DNA topoisomerase I (Top1) is a highly conserved enzyme composed of four domains: a positively charged N-terminus; a DNA binding/core domain that circumscribes duplex DNA; a non-conserved linker domain; and a C-terminal/catalytic domain. Top1 catalyzes changes in DNA topology by transient cleavage of a single DNA strand and the concomitant formation of a phosphotyrosyl linkage between the enzyme and the 3' DNA end. This covalent Top1-DNA complex is the binding site for camptothecin (CPT), which selectively inhibits religation of the cleaved DNA strand. CPT binding stabilizes the covalent complex, while the collision of replication forks with CPT-Top1-DNA adducts produces DNA lesions that induce cell death. Mutation of conserved residues in close proximity to the active site tyrosine, Tyr727 in yeast Top1 (yTop1) and Tyr 723 in human Top1 (hTop1), alters DNA cleavage-religation equilibrium, inducing drug independent lethality. yTop1T722 A mimics the action of CPT and decreases the rate of religation, while yTop1 $\mathrm{N} 726 \mathrm{H}$ increases the rate of cleavage. Even though both mutants increase the stabilization of the Top1-DNA covalent complexes, it is evident that distinct cytotoxic lesions are formed by the different responses induced by low level expression of these self-poisoning enzymes in isogenic yeast strains defective for the Rad9 DNA damage checkpoint, processive DNA replication, or ubiquitinmediated proteolysis. The core and C-terminal domains are connected by extended $\alpha$-helices (linker domain), which position the active site Tyr within the catalytic pocket. The physical connection of the linker domain with the Top1 clamp as well as linker flexibility $v$ affect enzyme sensitivity to CPT. Crystallographic data reveal a conserved Gly residue (located at the juncture between the flexible linker and C-terminal domains) that lies at one end of a short $\alpha$-helix, which extends to the active site Tyr covalently linked to the DNA. However, in the presence of drug, the linker is rigid and this $\alpha$-helix extends to include Gly and the preceding Leu, suggesting a dynamic interplay between active site $\alpha$ - helical structure and linker flexibility. In vitro and in vivo analyses of Gly 721 mutations in yTop1 demonstrate that charge and $\alpha$-helical propensity of amino acid side chains at position 721 impact active site architecture within the enzyme-DNA complex. In combination with the crystallographic data, these results implicate the conserved Gly 721 as a flexible hinge within the active site of Top 1 that enables linker flexibility and the structural alterations that accompany drug binding of the covalent enzyme-DNA complex. In vitro studies of the corresponding human mutant, hTop1G717 D, showed that this mechanism of increased sensitivity is conserved. However, by expressing hTop1G717 D in yeast cells the increased CPT sensitivity was not recapitulated. To determine the contribution of the divergent $\mathrm{N}$-terminal and linker domains to enzyme activity and in vivo CPT sensitivity, a series of chimeric enzymes, involving reciprocal swaps of the yeast and human $\mathrm{N}$-termini and linkers, were generated. The results demonstrate that the conserved core and C-terminal domains dictate the intrinsic enzyme sensitivity to CPT, while it is the functional interactions of the $\mathrm{N}$-terminal and linker domains that regulate enzyme activity in vivo.
\end{abstract}

\section{Document Type}

Dissertation

Degree Name

Doctor of Philosophy (PhD)

Program

Interdisciplinary Program

Research Advisor

Mary-Ann Bjornsti, Ph.D. 


\section{Keywords}

DNA topoisomerase I, camptothecin, enzyme architecture, domain flexibility, drug sensitivity, structural alteration

\section{Subject Categories}

Chemicals and Drugs | Enzymes and Coenzymes | Medicine and Health Sciences 


\title{
ENZYME ARCHITECTURE AND FLEXIBILITY AFFECT DNA TOPOISOMERASE I FUNCTION
}

\author{
A Dissertation \\ Presented for \\ The Graduate Studies Council \\ The University of Tennessee \\ Health Science Center
}

\begin{abstract}
In Partial Fulfillment Of the Requirements for the Degree

Doctor of Philosophy

From The University of Tennessee
\end{abstract}

By

Mariè van der Merwe

December 2007 


\section{DEDICATION}

I would like to dedicate this dissertation to my parents, Andries van der Merwe and Marita Esterhuyse for giving me roots so that I can stand strong and to my husband and best friend, Jan Kirsten, for giving me wings so that I can follow my dreams. 


\section{ACKNOWLEDGMENTS}

I would like to express my full gratitute to those who contributed to my dissertation research over the past years. First and foremost I thank my research advisor, Mary-Ann Bjornsti, for her willingness to train me, her guidance, encouragement and mostly her trust. She has been a great teacher in many aspects of my development as a scientist. I would also like to thank all the members of Mary-Ann's lab, past and present, for their friendship and support, morally and scientifically. A special thanks to Robert van Waardenburg for his willingness to help me use the Pymol sofware.

I also extent my gratitue towards my graduate comittee, Terry Cooper, Katsumi Kitagawa, Martine Roussel and Brenda Schulman for their guidance in overseeing my research and making appropriate suggestions. I believe our meetings had a crucial role in directing my research and I appreciate the time that it took from their busy shedules.

Kiran Kodali and Clive Slaughter in the Hartwell center played a critical role in

deciphering some of my data and I would like to thank them for sharing their expertise so patiently.

Last, but definitely not least, I would like to thank my family. Without their support none of this would have been possible. 


\begin{abstract}
DNA topoisomerase I (Top1) is a highly conserved enzyme composed of four domains: a positively charged N-terminus; a DNA binding/core domain that circumscribes duplex DNA; a non-conserved linker domain; and a C-terminal/catalytic domain. Top1 catalyzes changes in DNA topology by transient cleavage of a single DNA strand and the concomitant formation of a phosphotyrosyl linkage between the enzyme and the 3' DNA end. This covalent Top1-DNA complex is the binding site for camptothecin (CPT), which selectively inhibits religation of the cleaved DNA strand. CPT binding stabilizes the covalent complex, while the collision of replication forks with CPT-Top1-DNA adducts produces DNA lesions that induce cell death. Mutation of conserved residues in close proximity to the active site tyrosine, $\mathrm{Tyr}^{727}$ in yeast Top1 (yTop1) and $\mathrm{Tyr}^{723}$ in human Top1 (hTop1), alters DNA cleavage-religation equilibrium, inducing drug independent lethality. $\mathrm{yTop} 1 \mathrm{~T}^{722} \mathrm{~A}$ mimics the action of CPT and decreases the rate of religation, while yTop $1 \mathrm{~N}^{726} \mathrm{H}$ increases the rate of cleavage. Even though both mutants increase the stabilization of the Top1-DNA covalent complexes, it is evident that distinct cytotoxic lesions are formed by the different responses induced by low level expression of these self-poisoning enzymes in isogenic yeast strains defective for the Rad9 DNA damage checkpoint, processive DNA replication, or ubiquitin-mediated proteolysis. The core and C-terminal domains are connected by extended $\alpha$-helices (linker domain), which position the active site Tyr within the catalytic pocket. The physical connection of the linker domain with the Top1 clamp as well as linker flexibility
\end{abstract}


affect enzyme sensitivity to CPT. Crystallographic data reveal a conserved Gly residue (located at the juncture between the flexible linker and C-terminal domains) that lies at one end of a short $\alpha$-helix, which extends to the active site Tyr covalently linked to the DNA. However, in the presence of drug, the linker is rigid and this $\alpha$-helix extends to include Gly and the preceding Leu, suggesting a dynamic interplay between active site $\alpha$ helical structure and linker flexibility. In vitro and in vivo analyses of $\mathrm{Gly}^{721}$ mutations in yTop1 demonstrate that charge and $\alpha$-helical propensity of amino acid side chains at position 721 impact active site architecture within the enzyme-DNA complex. In combination with the crystallographic data, these results implicate the conserved Gly ${ }^{721}$ as a flexible hinge within the active site of Top1 that enables linker flexibility and the structural alterations that accompany drug binding of the covalent enzyme-DNA complex. In vitro studies of the corresponding human mutant, $\mathrm{hTop} 1 \mathrm{G}^{717} \mathrm{D}$, showed that this mechanism of increased sensitivity is conserved. However, by expressing hTop $1 \mathrm{G}^{717} \mathrm{D}$ in yeast cells the increased CPT sensitivity was not recapitulated. To determine the contribution of the divergent $\mathrm{N}$-terminal and linker domains to enzyme activity and in vivo CPT sensitivity, a series of chimeric enzymes, involving reciprocal swaps of the yeast and human N-termini and linkers, were generated. The results demonstrate that the conserved core and C-terminal domains dictate the intrinsic enzyme sensitivity to CPT, while it is the functional interactions of the N-terminal and linker domains that regulate enzyme activity in vivo. 


\section{TABLE OF CONTENTS}

CHAPTER 1. INTRODUCTION …..................................................................... 1

1.1 DNA topology and topoisomerases ................................................................. 1

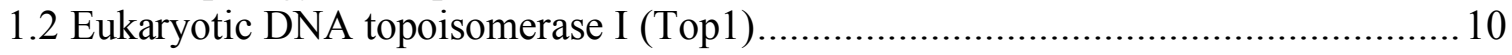

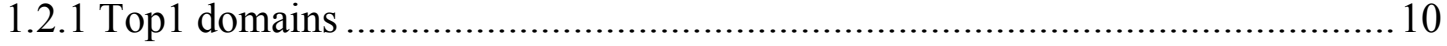

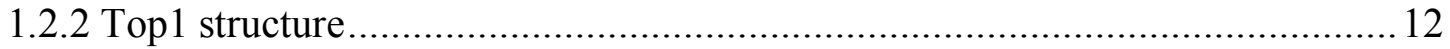

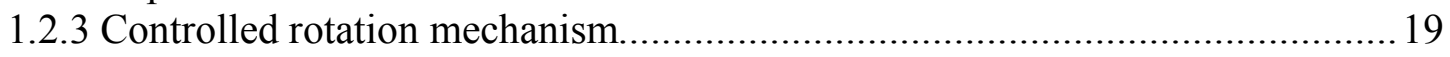

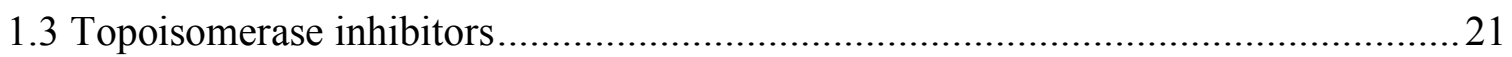

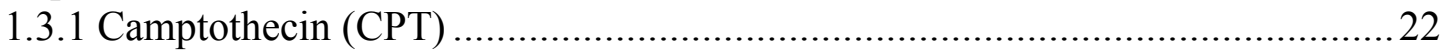

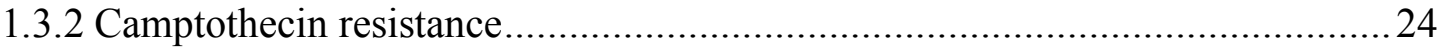

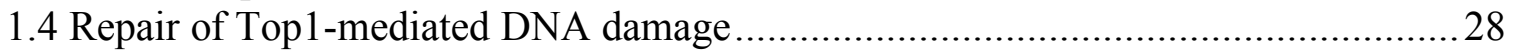

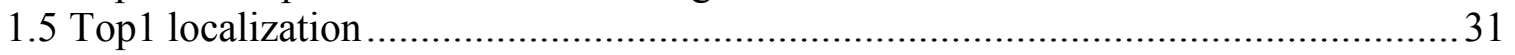

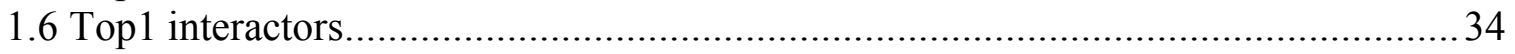

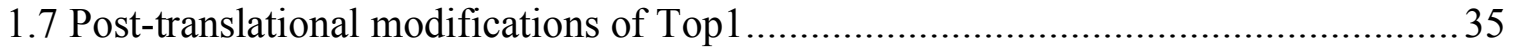

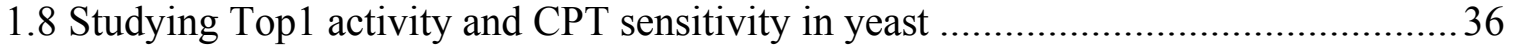

CHAPTER 2. SUBSTITUTION OF CONSERVED RESIDUES WITHIN THE ACTIVE SITE ALTER THE CLEAVAGE RELIGATION EQUILIBRIUM OF

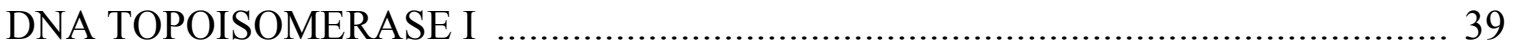

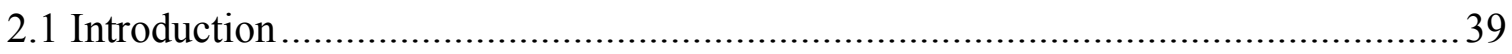

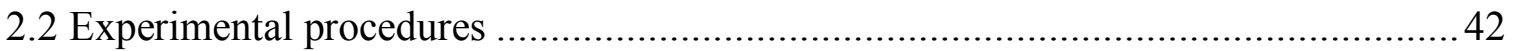

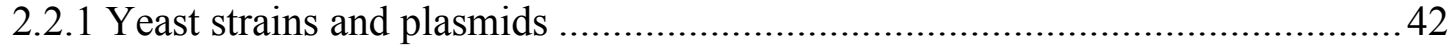

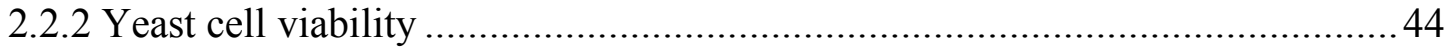

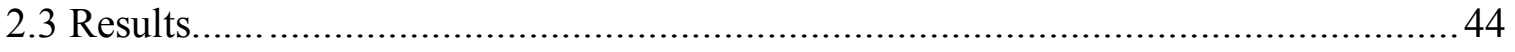

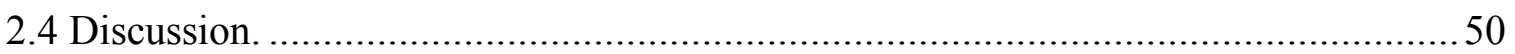

\section{CHAPTER 3. SUBSTITUTIONS OF GLY ${ }^{721}$ ALTER YEAST DNA} TOPOISOMERASE I ACTIVE SITE ARCHITECTURE AND ENZYME SENSITIVITY TO CAMPTOTHECIN .............................................................. 53

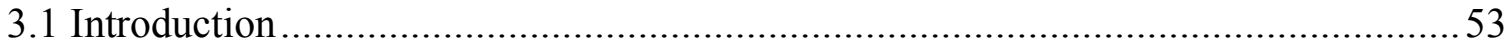

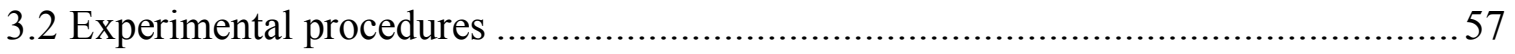

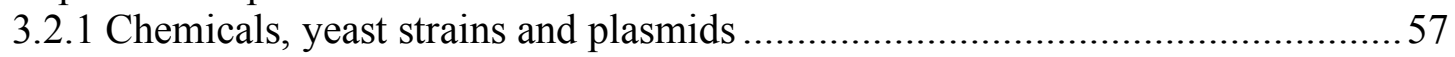

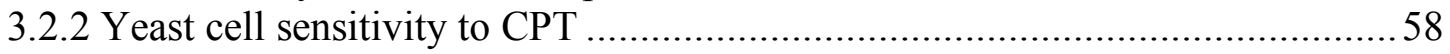

3.2.3 DNA topoisomerase I expression, purification and activity assays..................59

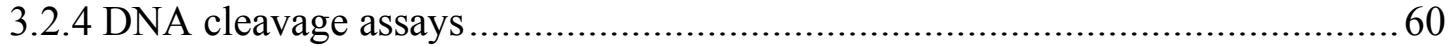

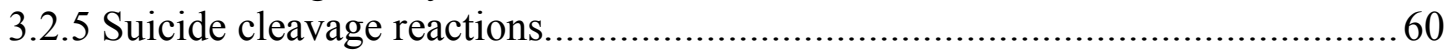




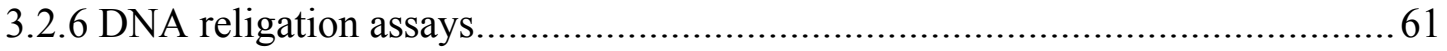

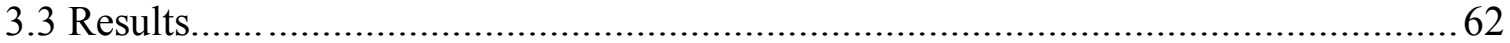

3.3.1 Substitution of Gly ${ }^{721}$ and Leu ${ }^{720}$ alter Top1 sensitivity to CPT .........................62 62

3.3.2 $\mathrm{Gly}^{721}$ mutations increase Top1 sensitivity to CPT in vitro ...............................69

3.3.3 Replacing Gly ${ }^{721}$ with acidic residues affect Top1 cleavage of DNA and enzyme architecture in the region preceding the active site Tyr .................71

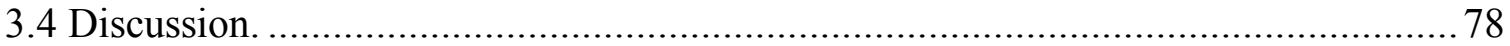

CHAPTER 4. DISTINCT STRUCTURAL DOMAINS REGULATE DNA TOPOISOMERASE I ACTIVITY AND SENSITIVITY TO CAMTOTHECIN..............84

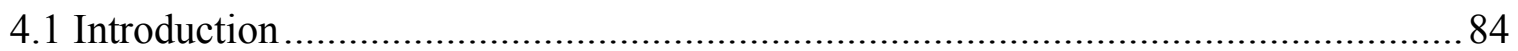

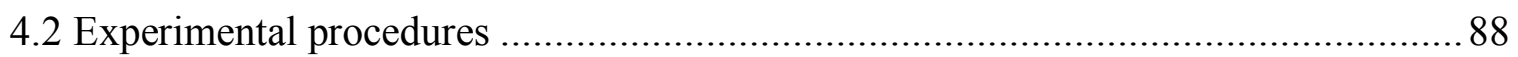

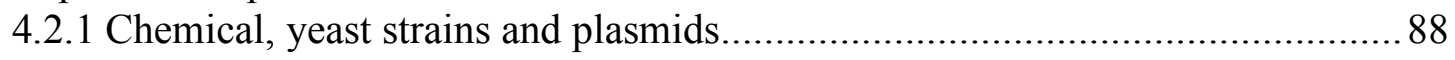

4.2.2 Yeast cell sensitivity to CPT ……………………....................................... 91

4.2.3 DNA topoisomerase I expression, purification and activity assays....................91

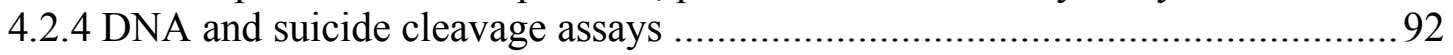

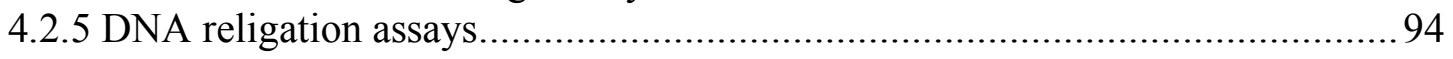

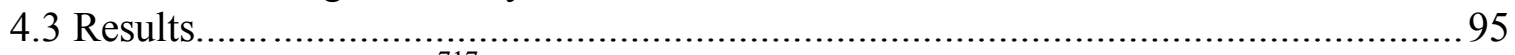

4.3.1 Mutation of $\mathrm{Gly}^{717}$ in human Top1 does not alter CPT-induced toxicity in yeast cells, yet enhances sensitivity to CPT in vitro .......................95

4.3.2 Mutation-induced changes in the DNA cleavage-religation equilibrium

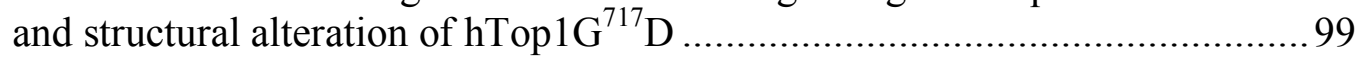

4.3.3 N-terminal and linker human/yeast Top1 chimeras ........................................ 109

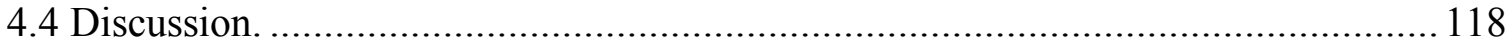

CHAPTER 5. IDENTIFICATION OF PROTEINS INTERACTING WITH TOP1 LINKER AND C-TERMINAL DOMAIN .............................................. 124

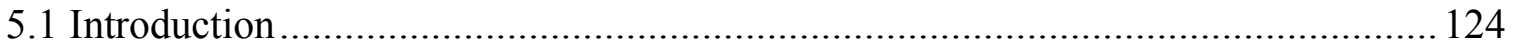

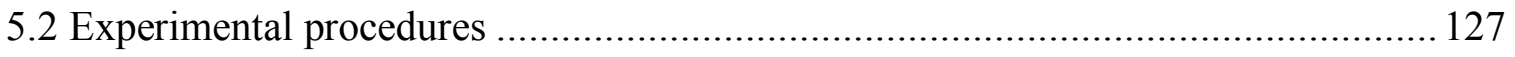

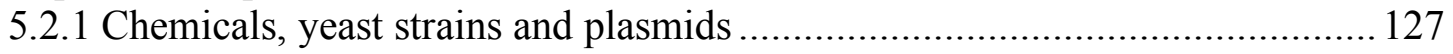

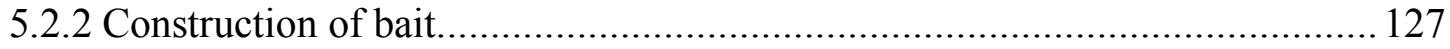

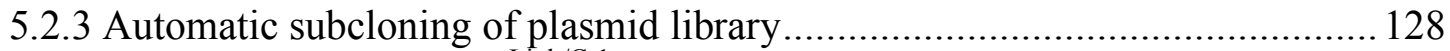

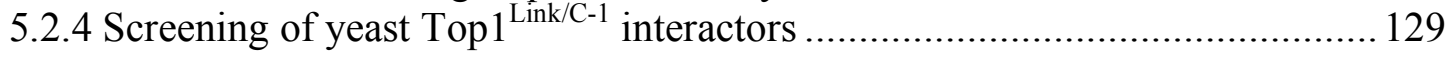

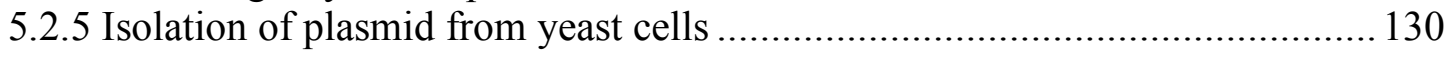

5.2.6 Confirmation of interaction, elimination of false positives and testing interaction with human Top1 linker and C-terminal domain................ 131

5.2.7 Detection of physical interaction between yTop $1^{\text {Link/C-1 }}$ and protein identified intwo hybrid screen using immunoprecipitations ................ 132

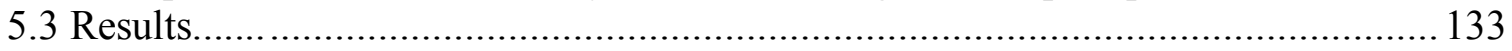

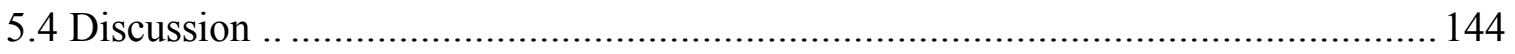


6.1. Mutations in the active site region of Top1 have been shown to induce distinct self poisoning activities.

6.2. Gly located in the transition region between the linker and the active site Tyr acts as a flexible hinge to alter active site geometry and linker flexibility and thereby influence CPT sensitivy.

6.3. The distinct linker domains play a role in regulating CPT sensitivity and enzyme catalysis in vivo.

LIST OF REFERENCES 166

VITA. 175 


\section{LIST OF FIGURES}

Figure 1.1. Amino terminal $67 \mathrm{kD}$ domain of E.coli DNA topoisomerase I. ..............4

Figure 1.2. $\quad 70 \mathrm{kD}$ C-terminal portion of human DNA topoisomerase I in non-covalent complex with a 22 base pair DNA duplex.

Figure 1.3. The dimerization of the $43 \mathrm{kD} \mathrm{N}$-terminal fragment of E.coli DNA Gyrase B subunit containing an ATPase domain and the DNA capture domain.

Figure 1.4. Human DNA Topoisomerase I (70kD) in non-covalent complex with a 22 base pair DNA duplex.

Figure 1.5. $\quad 70 \mathrm{kD}$ C-terminal portion of human DNA topoisomerase I (Topo70) in covalent complex with a 22 base pair DNA duplex

Figure 2.1. Distinct cellular responses to lesions induced by self-poisoning Top1 enzymes are suggested in cells deleted for the Rad9 DNA damage checkpoint.

Figure 3.1. Topotecan binding to the covalent Top1-DNA complex affects enzyme architecture and alters the geometry of the active site.

Figure 3.2. Mutation of $\mathrm{Leu}^{720}$ or Gly ${ }^{721}$ in yeast Top1 induces CPT resistance in vivo.

Figure 3.3. Substitution of $\mathrm{Gly}^{721}$ with residues containing $\gamma$-carbon branched side chains enhance cell sensitivity to $\mathrm{CPT}$.

Figure 3.4. Expression of Top $1 \mathrm{G}^{721} \mathrm{D}$ and $\mathrm{Top} 1 \mathrm{G}^{721} \mathrm{E}$ increases the cytotoxicity of CPT.

Figure 3.5. Top $1 \mathrm{G}^{721} \mathrm{D}$, Top $1 \mathrm{G}^{721} \mathrm{E}$ and $\mathrm{Top} 1 \mathrm{G}^{721} \mathrm{~N}$ exhibit increased levels of covalent complexes in the presence of CPT.

Figure 3.6. Truncated suicide substrates reduce the rate of DNA cleavage by Top $1 \mathrm{G}^{721} \mathrm{D}$.

Figure 3.7. Distinct patterns of Top1-DNA tryptic digests obtained in religation reactions suggest $G^{721}$ D-induced alterations in active site structure. 
Figure 3.8. Duplex DNA, 3' to the site of DNA scission, impacts

Top $1 \mathrm{G}^{721} \mathrm{D}$ active site architecture and the rate of

enzyme-catalyzed DNA religation.

Figure 3.9. A propensity for $\alpha$-helical structure at residue 721 enhances

Top1 sensitivity to CPT

Figure 4.1. The structure of a C-terminal $70 \mathrm{kD}$ fragment of human DNA

topoisomerase I (Topo70) in a noncovalent complex with a 22

base pair DNA duplex

Figure 4.2. Mutation of $\mathrm{Gly}^{717}$ to $\operatorname{Asp}\left(G^{717} D\right)$ or Gln $\left(G^{717} Q\right)$

enhanced hTop1 sensitivity to CPT in vitro, but not in yeast.

Figure 4.3. $\mathrm{hTop} 1 \mathrm{G}^{717} \mathrm{D}$ exhibits increased rates of DNA cleavage.

Figure 4.4. Substitution of Gly $^{717}$ with Asp increases the rate of

Top1-catalyzed DNA cleavage.

Figure 4.5. Tryptic digests of $\mathrm{hTop} 1 \mathrm{G}^{717} \mathrm{D}-\mathrm{DNA}$ complexes during DNA

religation suggest mutant-induced alterations in

active site structure.

Figure 4.6. The presence of duplex DNA downstream from the site of

DNA scission reduces the rate of hTop $1 \mathrm{G}^{717} \mathrm{D}$-catalyzed

DNA religation.

Figure 4.7. Co-crystal structure of TPT bound to the covalent

Topo70-DNA complex.

Figure 4.8. Reciprocal swaps of the N-terminal domains of yeast and human Top1 do not alter enzyme activity or yeast cell sensitivity to CPT.

Figure 4.9. The introduction of the yeast N-terminal domain suppresses the lethal phenotype of a human Top1-yeast linker domain chimera.

Figure 4.10. CPT sensitivity and sequence specificity are dictated by the core and C-terminal domains of Top1....

Figure 4.11. Catalytically inactive hTop1 containing a yeast linker is toxic to yeast cells.

Figure 4.12. Yeast Top $1 \mathrm{G}^{721} \mathrm{D}$ containing the human linker domain exhibits increased CPT sensitivity. 
Figure 5.1. Plasmids used for the construction of the bait and the prey.................. 134

Figure 5.2. Schematic representation of the two-hybrid system. ........................ 135

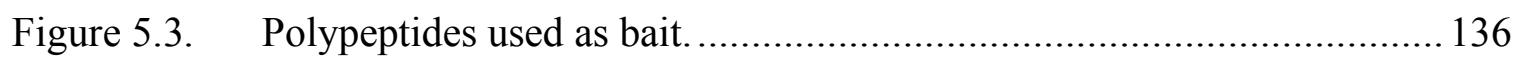

Figure 5.4. Ubc9 interacts with yTop $1^{\mathrm{Link} / \mathrm{C}-1}$ and hTop $1^{\mathrm{Link} / \mathrm{C}}$ equally well............. 142

Figure 5.5. Mrm1 and Pol30 physically interact with yTop1 $1^{\text {Link/C-1 }} \ldots \ldots \ldots \ldots \ldots \ldots \ldots \ldots . . . . . . . . .145$ 


\section{LIST OF TABLES}

Table 2.1. Transformation efficiency of isogenic top 1 yeast strains......................47

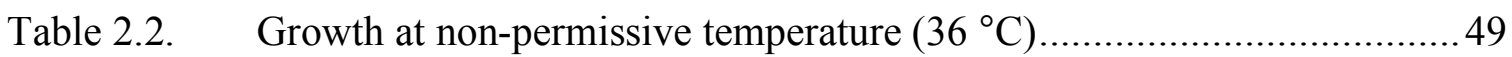

Table 4.1. Composition of yeast/human Top1 chimeras ................................. 90

Table 5.1. Proteins identified in the two-hybrid screen .................................... 139

Table 5.2. Distinct interactions with yTop $1^{\mathrm{Link} / \mathrm{C}-1}$ and hTop $1^{\mathrm{Link} / \mathrm{C}} \ldots \ldots \ldots \ldots \ldots \ldots \ldots . . . . . . . . . .144$ 


\section{LIST OF ABBREVIATIONS}

$\begin{array}{ll}\text { AD } & \text { Activation Domain } \\ \text { Ala } & \text { Alanine } \\ \text { Arg } & \text { Arginine } \\ \text { Asn } & \text { Asparagine } \\ \text { Asp } & \text { Aspartic acid } \\ \text { ATP } & \text { Adenosine triphosphate } \\ \text { CPT } & \text { Camptothecin } \\ \text { CPT-11 } & \text { Irinotecan } \\ \text { DBD } & \text { DNA Binding Domain } \\ \text { Dex } & \text { Dextrose } \\ \text { DMSO } & \text { Dimethyl sulfoxide } \\ \text { FRAP } & \text { Fluorenscence Recovery after Photobleaching } \\ \text { Gal } & \text { Galactose } \\ \text { Gln } & \text { Glutamine } \\ \text { Glu } & \text { Glutamic acid } \\ \text { Gly } & \text { Glycine } \\ \text { GFP } & \text { Green Fluorescent protein } \\ \text { His } & \text { Histidine } \\ \text { kD } & \text { kiloDalton } \\ \text { Leu } & \text { Leucine } \\ \text { Phe } & \text { Phenyalanine } \\ \text { rDNA } & \text { ribosomal DNA } \\ \text { SC } & \text { Synthetic complete } \\ \text { Tdp1 } & \text { Tyrosyl-DNA phosphodiesterase } \\ \text { Thr } & \text { Threonine } \\ \text { Top1 } & \text { DNA topoisomerase I } \\ \text { Tpt } & \text { Topotecan } \\ \text { Trp } & \text { Tryptophan } \\ \text { Tyr } & \text { Tyrosine } \\ \text { Ura } & \text { Uracil } \\ \text { Val } & \text { Valine } \\ \text { 3-AT } & 3 \text {-Amino-1,2,4-triazole } \\ & \end{array}$

xiii 


\section{CHAPTER 1: INTRODUCTION}

\subsection{DNA TOPOLOGY AND TOPOISOMERASES}

"Since the two chains in our model are intertwined, it is essential for them to untwist if they are to separate. Although it is difficult at the moment to see how these processes occur without everything getting tangled, we do not feel that this objection would be insuperable" (1).

In 1953 the scientific duo, Watson and Crick solved the structure of duplex DNA. They found that DNA exists as a double helix and that the bases on each strand were complementary to each other. This helical and complementary character of the DNA double helix represented an elegant solution to the complicated task of housing genetic information. It was further envisioned that to gain access to the information in this molecule, the two strands of the double helix must separate from each other and that the separation will have topological consequences (1). This problem leads to the fundamental need in all cells for a class of enzymes that is able to alter DNA topology, hence DNA topoisomerases. These enzymes are ubiquitous and found in all organisms, including viruses, bacteria, archaebacteria and eukaryotes (2).

All DNA topoisomerases alter DNA topology through a cleavage/religation mechanism that employs the chemistry of transesterification. Cleavage is initiated by the nucleophilic attack of the O-4 oxygen of the active site tyrosine on a phosphorous in the DNA backbone, resulting in a covalent phosphotyrosine linkage. Once DNA topology is 
altered, the cleaved strand is religated through a second transesterification reaction. The oxygen of the DNA hydroxyl group generated during the cleavage reaction now attacks the phosphorous of the phosphotyrosine linkage, breaking the covalent bond between the protein and the DNA and restoring the DNA backbone. These cleavage-religation cycles create transient enzyme-linked nicks in the DNA, such that another DNA strand or double helix can pass through $(3,4)$.

DNA topoisomerases are divided into two groups, type I and type II. Division of these families was based on the number of DNA strands cleaved during each reaction cycle and with which end of the broken strand the covalent linkage was formed in the enzyme-DNA intermediate. Type I enzymes are ATP-independent and transiently cleave only one strand of a DNA duplex. They are typically monomeric, with the exception of Top1 from Leishmania donovani promastigotes. This enzyme is reconstituted by two proteins encoded by separate genes ( $L d T O P 1 A$ and $L d T O P 1 B)$ located on separate chromosomes. Even though this enzyme is active as a dimer, it forms only one active site pocket containing the catalytic Tyr required for cleaving one strand of the DNA duplex (5). Type I enzymes are further divided into type IA and IB subfamilies. Type IA, such as bacterial DNA topoisomerase I and III, eukaryotic DNA topoisomerase III and reverse gyrase, are covalently attached to the 5'phosphate of the nicked strand, while type IB enzymes attach to the 3' phosphate of the cleaved DNA backbone $(2,6)$. Type IB includes nuclear topoisomerase I found in all eukaryotic cells, vaccinia virus DNA topoisomerase I and DNA toposiomerase V (2). In contrast to the nicking of a single strand by the type I enzymes, both strands of the DNA duplex are transiently broken by the type II enzymes. These enzymes, like type IA, are covalently linked to the 5' end of 
the cleaved DNA. Type II enzymes are also subdivided into type IIA and IIB. Type IIA includes the eukaryotic enzymes such as mammalian topoisomerase II $\alpha$, II $\beta$ and yeast DNA topoisomerase II. They function as homodimers forming two catalytic pockets. Type IIB enzymes such as archaeal DNA topoisomerase VI, have a distinct global architecture from the type IIA enzymes in that they are active as a heterotetramer consisting of two subunits of $\mathrm{A}$ and $\mathrm{B}$ each $-\mathrm{A}_{2} \mathrm{~B}_{2}$ - which are encoded by two separate genes. The amino and carboxy-terminal regions of type IIA enzymes are homologous to the A and B subunits respectively, such that the type IIB holo-enzymes also contain only two catalytic pockets. Despite the difference in composition of type IIB, its catalytic mechanism is similar to type IIA enzymes $(3,4,7,8)$.

Type IA enzymes catalyze DNA strand passage by an "enzyme bridging" mechanism, whereby the enzyme cleaves a single DNA strand and holding onto both DNA ends at the break, bridges the opening through which an intact strand is passed. It does not require an external energy source such as ATP, but $\mathrm{Mg}(\mathrm{II})$ is necessary for its relaxation activity.

Type IA topoisomerases first bind to a short stretch of single stranded DNA and therefore preferentially relaxes negatively supercoiled or underwound DNA. After binding to the single stranded DNA, the nucleophilic O-4 oxygen of the catalytic Tyr ( $\mathrm{Tyr}^{319}$ in E.coli Top1, indicated in pink in Fig. 1.1) attacks the scissile phosphate forming a covalent linkage with the 5'-end of the DNA, while non-covalently holding onto the 3'end. After cleavage, the domain covalently attached to the 5'end of the DNA (Domain III in Fig. 1.1) moves away from the base of the enzyme. (The base is indicated by Domain I.) This conformational change creates an opening (black arrow in Fig. 1.1) 


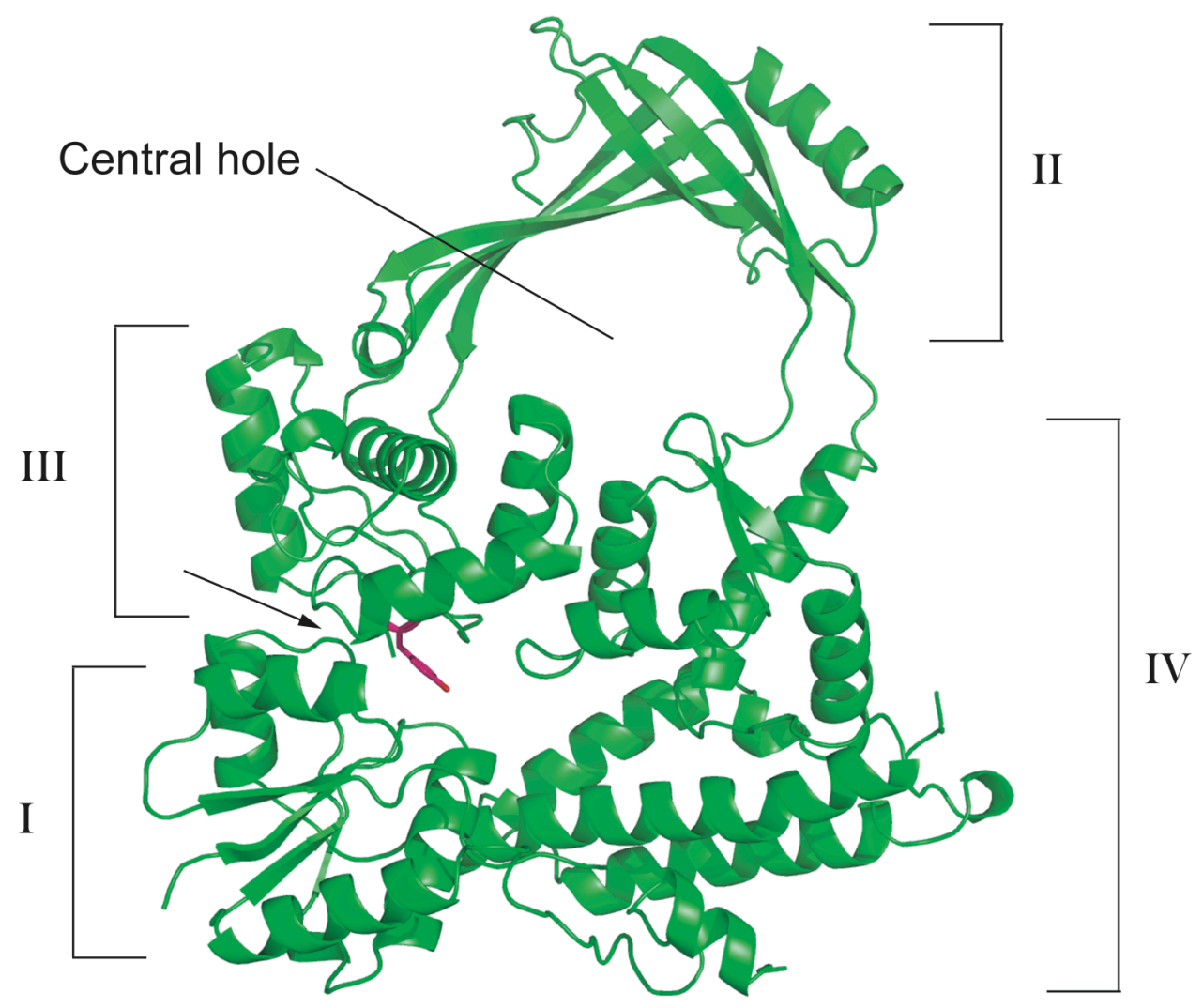

FIG. 1.1. Amino terminal 67kD domain of E.coli DNA topoisomerase I. [aa 32-590, pdb entry 1ECL (9)] The active site $\operatorname{Tyr}^{319}$ is indicated in pink. 
through which a single DNA strand can pass. Once the non-scissile strand has moved into the central hole of the enzyme, the clamp will close and the nicked strand is religated. For release of the non-scissile strand, the protein opens and closes a second time to conclude one catalytic cycle. This mechanism of relaxation elucidate the reason type IA enzymes change the linking number, the number of times the two strands of the duplex are wrapped around each other, in discreet steps of one $(3,7,10)$.

Single molecule experiments have confirmed this model of relaxation by type IA enzymes and further demonstrated that this enzyme is able to increase the linking number of positively supercoiled DNA providing that there is a short stretch of unpaired DNA (11). This demonstrates that the enzyme dictates directionality to the relaxation process.

Type IB topoisomerase is distinct from type IA in its mechanism of action as it relaxes DNA by strand rotation. This enzyme binds to duplex DNA and can relax positive or negative supercoils in the absence of energy cofactors or divalent cations. As it relaxes both positive and negative supercoils, the directionality of relaxation is determined by the torsional strain in the DNA backbone and not by the enzyme.

The protein first forms a non-covalent complex with duplex DNA by forming a tight clamp around it (Fig. 1.2). Cleavage occurs through a transesterification reaction in which the scissile phophodiester is attacked by the catalytic tyrosine of the enzyme, forming a DNA- (3'-phosphotyrosyl)-enzyme intermediate and releases the 5'-OH DNA end allowing it to rotate around a single phosphate bond in the non-scissile strand, relieving any torsional strain. After rotation the DNA 5'-OH group acts as a nucleophile attacking the phosphotyrosyl bond in the covalent intermediate, thereby restoring the DNA phosphodiester backbone $(4,7)$. As the religation rate is much faster 


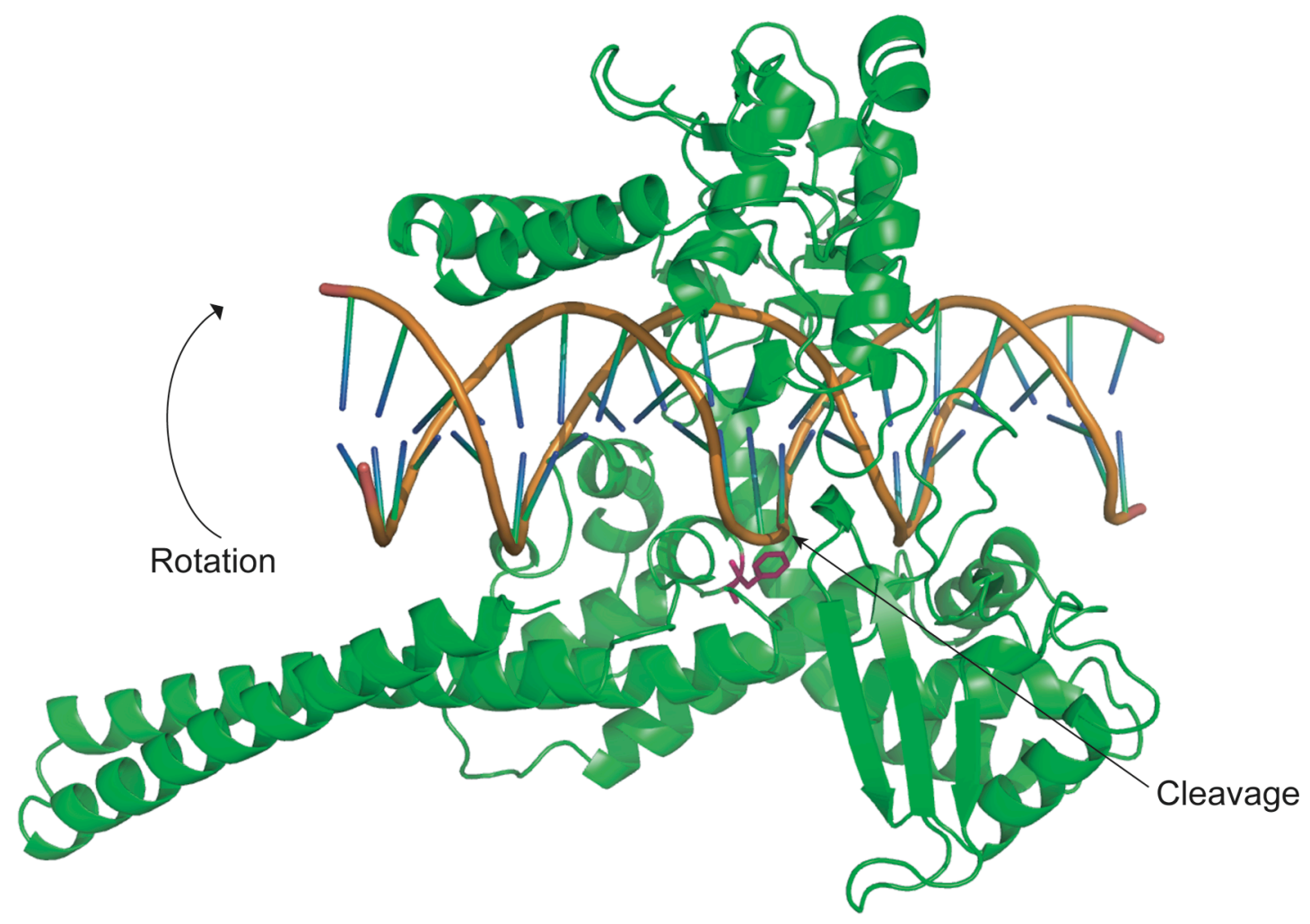

FIG. 1.2. $70 \mathrm{kD}$ C-terminal portion of human DNA topoisomerase I in non-covalent complex with a 22 base pair DNA duplex. [aa 175-765, pdb entry $1 \mathrm{~A} 36$ (12)] The enzyme is shown in green and the duplex DNA in orange. The active site $\operatorname{Tyr}^{723}$ was mutated to the Phe (indicated in pink). 
than the rate of cleavage, it ensures low steady-state levels of the covalent Top1-DNA complexes. (3). Single molecule experiments demonstrated that the rotation of the DNA inside the clamp is not free, but impeded by friction generated by clashes of the rotating strand with amino-acid side chains lining the protein-DNA interface. These experiments also demonstrated that the timing of religation depend on the proximity of 5'-OH end to the DNA-3'-phophotyrosine adduct and therefore multiple supercoils can be removed per cleavage-religation cycle (13). The tyrosine responsible for cleavage was mapped to the C-terminal domain (14) and by peptide sequencing and site-directed mutagenesis found to be located at position 727 in $S$. cerevisiae (15).

The type 1B enzymes are structurally and mechanistically distinct from all other known topoisomerases, but similar to a class of enzymes called site specific/Tyrosine recombinases. This family includes the bacteriophage HP1, $\lambda$ integrases, XerC/D, Cre, and Flp recombinases. They catalyze reciprocal DNA stand transfer reactions during the formation and resolution of four-strand Holliday junctions (3). The Tyr recombinases are mechanistically related to the type I topoisomerases as they also cleave a single strand of a DNA duplex through a nucleophilic attack of the active site Tyr, yielding a covalent(3'-phosphotyrosyl)-enzyme intermediate and a free 5'-OH strand. Despite a lack of sequence conservation, the $\mathrm{C}$-terminal catalytic domain of type IB topoisomerases and Tyrosine recombinases adopt a common fold composed of eight $\alpha$-helices and a threestrand anti-parallel $\beta$-sheet with the catalytic pentad occupying similar positions in the tertiary structures. Based on this conservation in structure and mechanism, it is proposed that these two classes of enzyme evolved from common ancestral DNA strand transferase. (16-19). Even though type IB enzymes can function as a strand transferase, as 
seen from in vitro religation assays, they religate the 5'-OH end that was created during the nucleophilic attack of the enzyme due to its proximity to the phosphotyrosyl bond.

Type II topoisomerases catalyze the ATP-dependent transport of an intact DNA double helix through another double helix. After enzyme binding, both strands of the DNA duplex are cleaved with a four base stagger, through coupled transesterification reactions between a pair of tyrosyl residues, indicated in pink in Fig. 1.3, located on each subunit of the dimer or the homologous $\mathrm{AB}$ complex. The tyrosine forms a phosphotyrosyl linkage with the 5 'end of the transiently broken DNA, leaving a pair of hydroxyl groups on the 3 ' recessed ends. A conformational change, driven by ATP binding, separates the ends of the cleaved duplex creating a gate in the DNA helix such that a second duplex can pass through the dimer interface. After passage, a second conformational change is initiated by the hydrolysis of ATP and ATPase domain reopens. The double strand break is resealed by a second transesterification reaction between the 3'-OH ends and the phosphotyrosyl bond to restore the continuity of the DNA. Due to this mechanism the type II enzymes change the net linking number in steps of two $(7,8)$.

Six topoisomerases exist in humans, two members of each subfamily: type IA, IB and IIA (IIB is only found in archea). Each of these enzymes has a unique function in altering DNA topology. Top IB acts as a swivel during replication and transcription to relieve helical tension resulting from the movement of the replication fork or transcription machinery, while Top II specifically decatenates DNA during mitosis. There are also overlapping functions between these enzymes as Top II is able to complement the function of Top 1B in yeast cells devoid of Top 1B (20). 


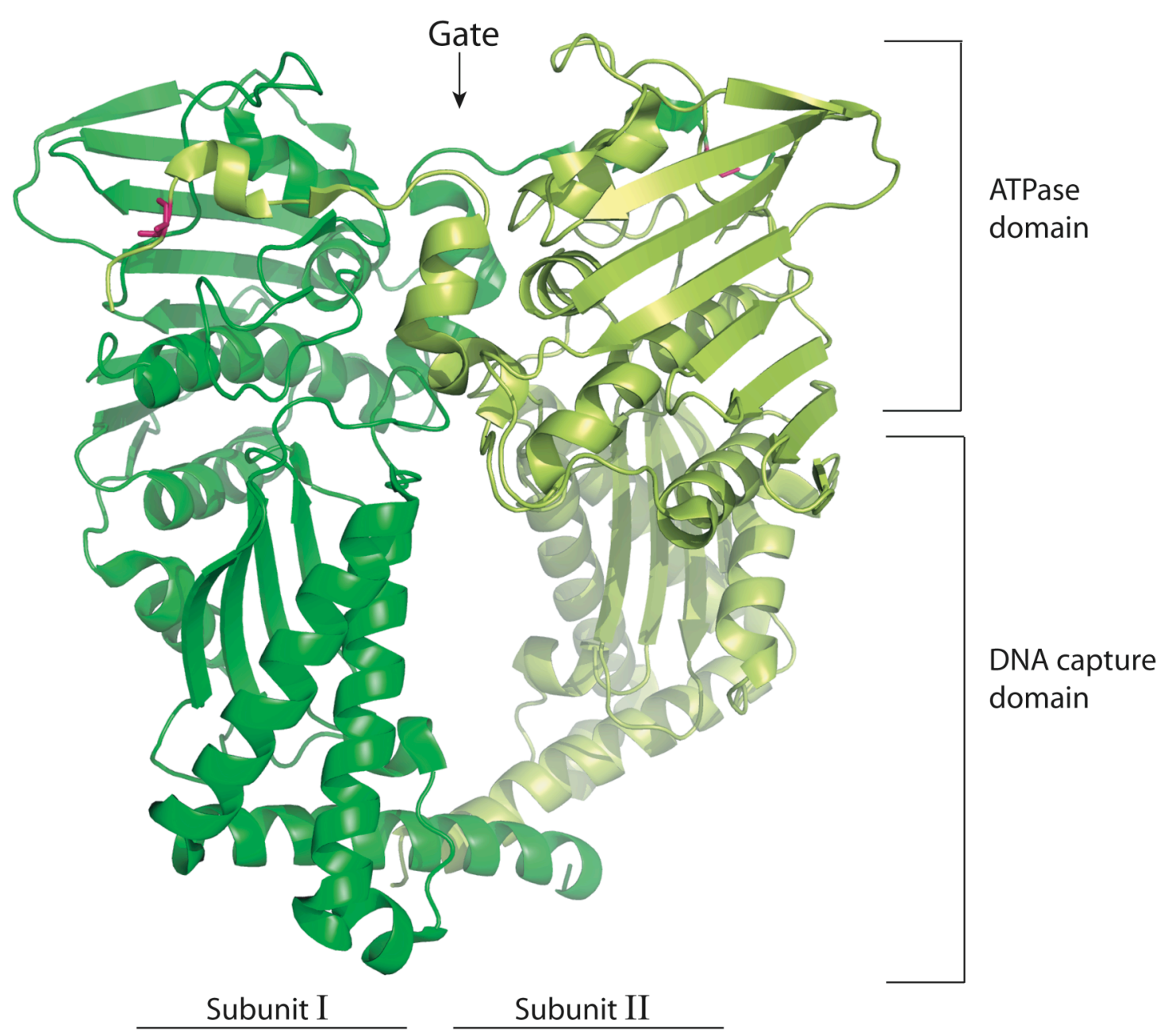

FIG. 1.3. The dimerization of the $43 \mathrm{kD}$ N-terminal fragment of E.coli DNA Gyrase B subunit containing an ATPase domain (aa 1-220) and the DNA capture domain (aa 221-392). [pdb entry 1EI1 (21)] The active site Tyr ${ }^{5}$ are mutated to Ser indicated in pink. 
For this study we focused on eukaryotic DNA topoisomerase I (Top1), which is a Type IB enzyme.

\subsection{EUKARYOTIC DNA TOPOISOMERASE I (Top1)}

In higher eukaryotes, Top1 is an essential enzyme as it is required for viability in mice during early embryogenesis. TOP 1 knockout mice are unable to survive past the 416 cell stage suggesting that this enzyme is required early after fertilization (22). Studies with Drosophila melanogaster showed similar results, demonstrating that Top1 is required for embryonic cell proliferation (23). Developmental expression profiles also suggested that Top1 is necessary during several stages of fly development, but especially 6-12 $\mathrm{h}$ post fertilization, when rapid DNA synthesis takes place (24). Although Top1 is essential for multicellular organisms, yeast cells lacking Top1 are viable, but show a slight slow growth defect and increased recombination at the tandemly repeated ribosomal DNA (rDNA) locus $(25,26)$.

\subsubsection{Top1 domains}

Top1 is a multi domain protein that can be subdivided into four distinct regions based on sequence conservation, sensitivity to limited proteolysis and fragment reconstitution experiments $(27,28)$. The $\mathrm{N}$-terminal domain is hydrophilic, unstructured and highly protease-sensitive. This domain is not well conserved in terms of primary sequence and size, but all nuclear Top1's have an N-terminal domain rich in charged residues. For example, the $24 \mathrm{kD}$ human Top1 N-terminal domain consists of $72 \%$ 
charged amino acids. This domain is dispensable for in vitro relaxation activity (29-31), but is required for in vivo Top1 nuclear localization as it contains the nuclear localization signals (NLS). The N-terminus is also important for protein-protein interactions (32-36). An intriguing feature of this domain is that while in the human enzyme, all the nuclear localization signals are located in the N-terminus, in yeast Top 1 there is also a canonical NLS in the linker domain. The N-terminus of human Top1 is much larger than that of yeast Top1, while the linker domain of yeast Top1 is much larger than that of human Top1, suggesting that there might be compensatory changes between the two domains and that they act as a functional unit.

None of the Top1 crystal structures solved to date contains an N-terminal domain. Therefore, the structure and also the spatial location of this domain relative to the core, linker and C-terminus are not known. It has been suggested by experiments using the $\mathrm{N}$ terminal truncated form of human Top1 that there is interaction of the N-terminus with DNA downstream of the cleavage site (37).

The N-terminal domain is followed by the highly conserved core domain containing all the catalytic residues; $\mathrm{Arg}^{488}, \mathrm{Lys}^{532}, \mathrm{Arg}^{590}$ and $\mathrm{His}^{632}$ (human Top1), except for the active site tyrosine required for DNA cleavage. The catalytic tetrad located in the core acts to position the scissile phosphate during the nucleophilic attack and stabilizes the transition state of the reaction through interactions with the non-bridging oxygen atom of the 3'-phosphate of the cleaved strand (38).

A protease sensitive linker connects the core to the highly conserved C-terminal domain which contains the nucleophilic tyrosine (15). The linker domain is poorly conserved in sequence and length, yet it is thought that the linker of all monomeric Top1 
enzymes forms a coiled-coil structure. Individually purified fragments consisting of the core and C-terminal domain (missing the linker region) tightly associate in a 1:1 ratio to reconstitute an active enzyme that exhibits plasmid relaxation activity and sequence specificity similar to full length Top1 (39). This experiment shows that the linker domain is dispensable for relaxation by the reconstituted enzyme, although its removal affects enzyme processivity $(12,30,39)$. It also demonstrates that the core and the catalytic $\mathrm{Tyr} /$ C-terminal domains represent the minimal requirement for DNA relaxation in vitro.

\subsubsection{Top1 structure}

The first crystal structures of a C-terminal portion of human Top1 were solved 1998 by the Champoux group. Stewart et al. solved the structure of an N-terminally truncated, catalytically inactive $70 \mathrm{kD}$ form of human Top1 composed of residues Lys ${ }^{175}$ to $\mathrm{Ph}^{765}$ (Topo70) non-covalently bound to DNA (Fig. 1.4), while Redinbo et al. solved the structure of a reconstituted enzyme consisting of the purified $58 \mathrm{kD}$ core domain (residues 175-659) and the $6.3 \mathrm{kD} \mathrm{C}$-terminal domain (residues 713 to 765 ), covalently and non-covalently bound to DNA. In all cases the protein was crystallized in the presence of 22 basepair duplex DNA that contains the high affinity Top1 binding site from Tetrahymena thermophilus (40). No crystals formed in the absence of this DNA.

The crystallographic results revealed a bi-lobed structure that clamps tightly around the DNA duplex forming a central DNA-binding pore, 15-20§ in diameter. The core and C-terminal domains associate closely with each other to completely circumscribe duplex DNA, confirming biochemical data that demonstrated a tight 1:1 binding between the core and C-terminal domain (39). 


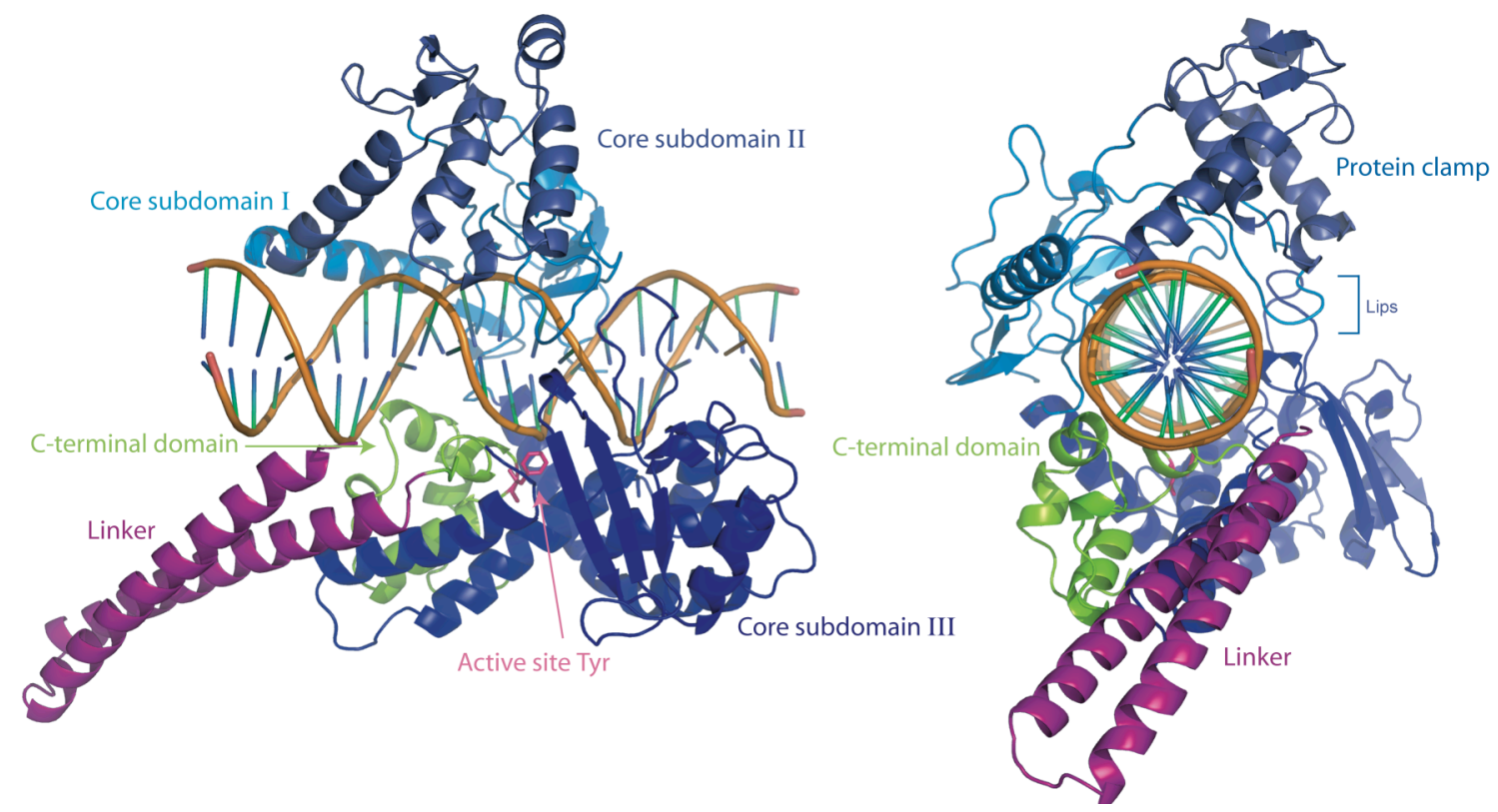

FIG. 1.4. Human DNA Topoisomerase I (70kD) in non-covalent complex with a 22 base pair DNA duplex. [Pdb entry 1A36 (12)] Viewed perpendicular to the pore, Core subdomain I, II and III are shown in blue, linker in purple and $\mathrm{COOH}$-terminal domain in green (left). On the right is a view of the enzyme down the pore, rotated $90^{\circ}$ about the vertical axis relative to the structure on the left. 
Core subdomains I and II fold together to form the top lobe of the enzyme, also referred to as the "cap". The cap is structurally characterized by two long "nose cone" helices that protrude away from the body of the enzyme. Core sub domain III and the C-terminal domain form the bottom lobe of the enzyme and are linked to the cap by a long alpha helix on the one side of the DNA. On the opposite side of the duplex, opposable "lip" domains (one loop from subdomain I and two loops from subdomain III) interact through a salt bridge to connect the cap to the bottom lobe. The majority of the interactions between the protein and DNA involve the amino acid side chains or primary amines with the DNA phosphates over a ten base pair stretch. The limited base specific contacts are consistent with the lack of Top1-DNA sequence specificity. For enzyme binding and release, the clamp is required to open and close which involves breaking the interaction between the two lip domains and lifting the cap away from the base of the enzyme.

As there is no crystallographic data available for Top1 in the absence of DNA, it is not known if Top1 forms a closed clamp when it is not bound to DNA and moreover, as the DNA is packed so tightly inside the central pore of the enzyme, the crystal structures fail to elucidate how rotation is achieved in the context of the covalent complex. To address these questions a mutant form of human Top1 was generated that forms reversible disulfide bonds between opposable lips such that the clamp can be locked in a "closed" conformation. Based on structure-modeling studies mutations $\mathrm{G}^{365} \mathrm{C}$ and $\mathrm{S}^{534} \mathrm{C}$ were introduced into full length human Top1. This mutant demonstrated that Top1 can form a clamp in the absence of DNA and furthermore, when the clamp is tightly locked around the DNA duplex there is no strand rotation, suggesting that protein flexibility facilitate DNA rotation (41). 
Two extended $\alpha$ helices constitute the linker domain. They form an anti-parallel coiled-coil projecting away from the globular body of the enzyme as seen in the crystal structures of Topo70. This domain contacts the rest of the protein only between the last helix of the core domain and the C-terminal portion of second linker helix. As there is no crystallographic data available for the N-terminal domain, it is not known if there is any interaction of this domain with the linker.

The linker helices are connected to each other through three interhelix salt bridges and nine hydrophobic pair-wise interactions. The fact that there are so few contacts between the linker and body of the protein, gives this domain a high degree of flexibility as revealed by multiple non-isomorphous crystal structures (42), but again, as Topo70 does not contain the N-terminal domain, it is not known what effect this domain has on linker flexibility. The region of the linker facing the DNA (proximal face) has a large net positive charge, with only two residues $-\mathrm{Lys}^{650}$ and $\mathrm{Arg}^{708}$ - hydrogen bonding with phosphates in the DNA backbone downstream of the cleavage site. The two interacting residues are located in the coiled-coil structure in close proximity to the core, and are conserved as either arginine or lysine in all type IB topoisomerases.

An anti-parallel, positively charged, coiled-coil structure, similar to the linker, is repeated in the "nose-cone" of the cap. It also projects away for the body of the protein, facing, but not interacting with, the DNA duplex. Due to their location and charge, it has been postulated that the "nose cone" and the linker play a role in the rotation of the DNA downstream from the cleavage site $(12,27)$. More recent data suggest that there is a conformational change in the protein such that the clamp "breaths" during DNA rotation and therefore the role of the "nose cone" and linker during rotation is not well understood 
(41). Also, single molecule experiments demonstrated friction between the protein and the rotating DNA, with human Top1 rotating slower than vaccinia Top1 (13). But, because vaccinia Top1 does not completely circumscribes duplex DNA and does not contain a linker domain, it is not understood how much of the friction is generated by the linker.

Cleavage occurs after the enzyme binds to duplex DNA, interacting with regions both upstream $(-7$ to -1$)$ and downstream $(+6$ to +11$)$ of the cleavage site. The cleavage site is between DNA residues -1 and +1 on the scissile strand $(43,44)$. The highest concentration of protein-DNA phosphate contacts is clustered around the 5 base pairs upstream (-5 to -1$)$ of the cleavage site. There is only one base-specific interaction between the enzyme and its DNA substrate, namely catalytic residue Lys ${ }^{532}$ contacting -1 thymidine $(\mathrm{T})$ nucleotide through the O-2 carbonyl oxygen of the base. Mutation of $\mathrm{T}$ at the -1 position has been shown to reduce the ability of the enzyme to bind to the DNA (45). Top1 has a sequence preference for nucleotides from positions -4 to -1 as follows: $5^{\prime}-(\mathrm{A} / \mathrm{T})(\mathrm{G} / \mathrm{C})(\mathrm{A} / \mathrm{T})(\mathrm{T})-3^{\prime}$ ' with covalent linkage at the -1 thymine residue (46-48). Even though Top1 is highly promiscuous with respect to sequence specificity, a strong cleavage site for all eukaryotic Top1's was found in the rDNA repeats of Tetrahymena thermophilus (40). This sequence contains a $\mathrm{T}$ at the -1 position and formed the basis for the 22 base pair oligo used in the crystallographic analyis $(12,27,38,40)$.

Many studies tried to map the putative binding sites for Top1 on double stranded DNA and found that sequence specificity is not the main determinant of Top1 localization on the DNA, but that structural features such as local helical twist play a more prominent role (49). This is underscored by the fact that the high affinity cleavage 
site for Top1 is flanked by two oligo (dA)-(dT) tracts which are associated with a stable curvature in the DNA (50). Moreover, Top1 preferentially binds to superhelical over relaxed DNA (51), implying that Top1 has a higher affinity for bent DNA and therefore senses writhe rather than twist. The linking number of a DNA molecule is represented by both twist and writhe, twist being the number of helical turns in the DNA and writhe is the number of times the double helix crosses over itself. As writhe is independent of the sign of supercoiling, it is consistent with the observation that Top1 binds equally well to negative and positive supercoiled DNA (52). It was also shown by electron microscopy that Top1 is associated with nodes where two duplexes cross each other, again suggesting that a structural feature of curved DNA is important for Top1 binding (52-54).

Eukaryotic DNA topoisomerase I is the sole target of the anticancer drug camptothecin (CPT) $(6,55-57)$. This drug has been difficult to study as it is an uncompetitive inhibitor and only transiently binds to the protein-DNA covalent complex, where it prevents religation (58). The crystal structure of Top1 covalently linked to DNA in the presence or absence of topotecan, an analog of CPT, was solved by Staker et al. using a suicide DNA substrate containing a 5'-bridging phosphorothiolate (Fig 1.5). Top1-catalyzed cleavage of this substrate generates a 5'-sulfhydryl, instead of a 5'hydroxyl, which acts as a weaker nucleophile in the religation reaction. These structures demonstrated that topotecan intercalates at the site of DNA cleavage, mimicking a DNA basepair. It is stabilized by base-stacking interactions with the -1 and +1 base pairs and one direct hydrogen bond between Top1 residue $\mathrm{Asp}^{533}$ and the 20(S) hydroxyl of the drug. The reduction in religation is elucidated by the fact that binding/intercalation of the drug displaces the reactive $5^{\prime} \mathrm{OH}$ end of the cleaved strand $3.6 \AA$ away from the catalytic 


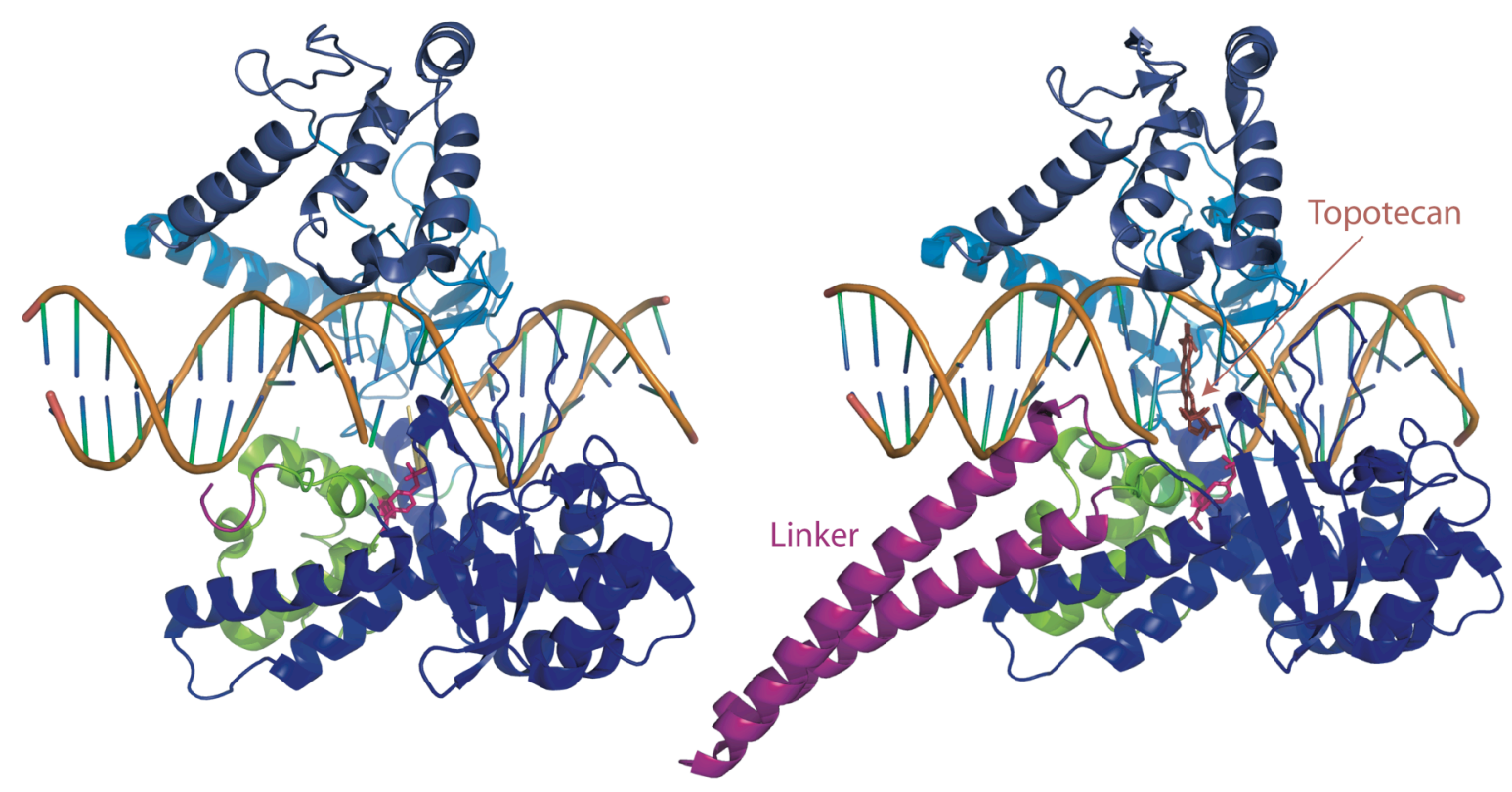

FIG. 1.5. $70 \mathrm{kD}$ C-terminal portion of human DNA topoisomerase I (Topo70) in covalent complex with a 22 base pair DNA duplex (left). [Pdb entry $1 \mathrm{~K} 4 \mathrm{~T}$ (59)] The figure on the right is Topo70 in complex with the CPT derivative, Topotecan [Pdb entry $1 \mathrm{~K} 4 \mathrm{~S}(59)]$. 
tyrosine, thereby impeding the religation step (59).

The most obvious difference between the crystal structures of the Top1-DNATPT ternary complex and the covalent Top1-DNA complex resides in the visibility of the linker, as seen in Fig. 1.5. In the binary structure, the linker domain residues $\left(\mathrm{Gln}^{633}-\right.$

$\mathrm{Gln}^{697}$ ) are not visible in the electron density, indicating movement of this coiled-coil structure. This is in accordance with studies by Redinbo et al., demonstrating that the linker domain showed the greatest degree of flexibility as revealed by the nonisomorphous crystal structures (60). In contrast to the nondrug-bound protein, the crystal structure of the ternary complex exposes the linker as an anti-parallel coiled-coil, similar to the linker structure in the non-covalently bound Top1-DNA complex. This suggests the posibility that binding of the drug alters enzyme conformation such that there is a decrease in linker flexibility. However, the effect of crystal packing on linker visibility is not known.

\subsubsection{Controlled rotation mechanism}

Based on structural and biochemical data, it was proposed that Top1 relaxes supercoils by the swiveling of the scissile strand downstream of the cleavage site about a phosphate bond. The co-crystal structures demonstrated that there is inadequate space for the DNA to rotate unhindered around the non-scissile strand without contacting the cap or the linker domain, and therefore a 'controlled rotation' model was suggested. This model proposes that after cleavage, the torsional stress in the supercoiled DNA drives rotation of DNA downstream of the cleavage site relative to the region of the duplex held by the protein clamp and that the rotation of the DNA is controlled by steric clashes of 
the DNA within the tight Top1 clamp $(12,39)$. Even though the "controlled rotation" model was supported by the crystallographic data, it failed to elucidate how rotation is achieved in the context of the covalent complex. Single molecule experiments measuring in real-time the velocity of DNA extension during supercoil removal demonstrated that vaccinia Top1 has a slower DNA rotation rate compared to the unhindered rotation of a nicking enzyme, substantiating the idea that steric clashes between the DNA and the amino acids of the protein-DNA interface affects rotation. Moreover, mutating $\mathrm{Tyr}^{70}$ of vaccinia Top1, such that the amino acid side chain extending into the DNA-protein interface, is smaller, increased the rate of DNA rotation (13). Woo et al., using a mutant Top1 protein that locks around duplex DNA, demonstrated that Top1 can still cleave DNA in the context of the locked clamp, but that locking the clamp around the DNA prevents DNA unwinding. Thus the protein clamp has to open for rotation to occur, thereby reducing steric clashes (41).

The linker appears to play a role in the relaxation process as a functional linker prolongs the lifetime of the covalent intermediate (61). Both the N-terminus and the linker are extremely sensitive to proteolysis, but binding of the enzyme to duplex DNA results in proteolytic protection of only the linker, indicating that the linker is able to interact with the DNA substrate (30). This is further substantiated by studies using fragment complementation of the core and C-terminal domain containing no linker. The reconstituted enzyme was comparable to wild-type in relaxation activity and sequence specificity, but differed in its affinity for DNA as the topoisomers is more distributive in a plasmid relaxation assay, again suggesting interactions between the linker and DNA (39). 
The structure and function of the linker was further characterized by fragment complementation, now using the core and a C-terminal fragment that extends to include the linker domain. By disconnecting the linker from the core, the folding and/or orientation of the linker was altered as assayed by gel filtration. Proteolytic studies demonstrated that the linker is protected from proteolysis when Top1 is non-covalently or covalently bound to DNA. Contrary to the wild type human Top1, proteolysis of the reconstituted enzyme containing a disconnected linker showed that this domain is not resistant to proteolysis, either when the enzyme is non-covalently or covalently linked to duplex DNA. Moreover, the reconstituted enzymes have reduced CPT sensitivity in cleavage assays, with no difference between the reconstituted enzyme containing a linker or not, implying that detaching the linker from the core rendered the linker nonfunctional. These results demonstrate that a detached linker differs in conformation/orientation and is unable to bind DNA downstream from the cleavage site and furthermore, that a functional linker is necessary for optimal CPT sensitivity (61). This was further underscored by a mutation in the linker domain $-\mathrm{A}^{653} \mathrm{P}$ - that is predicted by molecular modeling to increase linker flexibility. This mutation induced CPT resistance, again demonstrating that a functional linker is required for $\mathrm{CPT}$ sensitivity (62).

\subsection{TOPOISOMERASE INHIBITORS}

There exists significant medical interest in topoisomerases as they constitute the cellular targets of naturally occurring antimicrobial and anticancer drugs. Among the 
antimicrobial agents are the fluoroquinolones, such as ciprofloxacin, which target bacterial DNA gyrase and is the most active broad spectrum spectrum oral antibiotic in clinical use (63). Top 2 isozymes are targeted by anthracyclines (doxorubicin), Genistein, Amsacrine (m-AMSA), Mitoxantrone and epipodophylotoxins (Etoposide) (64). Etoposide is one of the most commonly prescribed anticancer drugs and is front line therapy small-cell lung cancer and a variety of leukemias, lympomas, and germ-cell malignancies (65).

\subsubsection{Camptothecin (CPT)}

Top1 is the sole target of the anticancer drug Camptothecin (CPT) $(6,55,57,66$, 67). This molecule is a naturally occurring alkaloid found in the Chinese plant, Camptotheca accuminata. It was first identified in 1960s in a screen of plant extracts for antineoplactic drugs. Even though CPT showed promising anticancer activity, its toxicity in phase II clinical trails precluded further use (68). There was renewed interest in CPT when Top1 was found to be the cellular target of this compound. Biochemical studies with mammalian Top1 and the finding that the Top1 purified from a CPT resistant human lymphoblastic leukemia cell line was resistant to CPT, implied that Top1 is the target of the alkaloid $(67,69-71)$. Moreover, studies using yeast cells (S. cerevisiae and S. pombe) demonstrated that removing Top1 rendered the cells viable, but CPT resistant. CPT sensitivity can be augmented by increasing the Top 1 protein levels $(55,57,66)$. It was also shown that CPT specifically binds to Top1 in the presence of DNA (58) and that the stabilization of the Top1-DNA intermediate caused single strand DNA breaks (6). 
Water-soluble derivatives of Top1 - Topotecan (TPT) and Irinotecan (CPT-11) have been approved for clinical use by the FDA and are among the most effective compounds tested for treating human cancer (72). These CPT analogs are pentacyclic structures with a lactone (closed ring) moiety in the E-ring. The lactone ring undergoes reversible, $\mathrm{pH}-$ dependant hydroysis to the hydroxy acid (open ring) and although the hydroxy acid form predominates at physiological $\mathrm{pH}$, the lactone is the pharmacologically active form (73). TPT is a 10-hydroxyl modification of CPT and is approved for treatment of metastatic ovarian and small cell lung cancer, as well as myeloid malignancies. CPT-11 is a prodrug, which is converted to the active compound SN-38 by plasma and cellular carboxylesterases. It is approved for use in treatment of metastatic colon and rectal carcinoma (74).

Camptothecin and its derivatives reversibly bind to the transient covalent Top1DNA intermediate, intercalating between the two bases of the cleaved strand and blocking the religation step. Although they are generally referred to as Top1 inhibitors, these compounds convert Top1 into a cellular poison by preventing religation of the cleaved strand and thereby inhibiting the dissociation of the protein from DNA. These Top1-DNA linked complexes are ubiquitous throughout the genome due to the enzymes' normal activity, but because religation is faster than cleavage these cleavage complexes are transient and difficult to detect. Therefore, by preventing religation, CPT increases the lifetime of this covalent intermediate $(57,67,75)$. Other DNA modifications such as single nucleotide gaps, base mismatches, guanine methylation or oxidative damage also lead to persistent Top1- DNA covalent complexes (76-78) These protein-linked DNA 
lesions themselves are insufficient for cell killing, however, in the presence of advancing replication forks, they are converted to irreversible cytotoxic DNA lesions (2). This is in agreement with the observation that, even though CPT can stabilize the ternary complex

in all stages of the cell cylce, its cytotoxic effect requires ongoing DNA replication (79). Cytotoxicity is suppressed when replication is inhibited with aphidicholin in both mammalian and yeast cells (80-82). Moreover, in vitro replication studies suggest that the replication machinery has a key role in the conversion of the Top1-DNA lesion to irreversible cytotoxic DNA damage (83). Analysis of the abnormal replication intermediates in the SV40 cell-free replication system suggested that that collision between the replication fork and the stabilized complex generates biochemically distinguishable events. Namely, formation of a double strand break, irreversible arrest of fork movement and formation of irreversible Top1-DNA covalent adducts. The structure of these intermediates depends on the relative orientation of covalently-linked protein and the advancing replication fork. For these events to occur, Top1 has to be covalently linked to the strand that is used as the template for leading strand DNA synthesis. This was supported by in vivo studies analyzing replication intermediates from tissue culture cells treated with CPT (84).

\subsubsection{Camptothecin resistance}

Resistance to topoisomerase I inhibitors results form various mechanisms including decrease in total cellular Top1 activity, structural mutation of Top1 and altered cellular accumulation of the drug (73). Despite the fact that submicromolar concentrations are necessary to destroy proliferating neoplastic cells, the clinical the 
response rate in adults is only $30 \%$, with effects being transient and resistance occurring frequently (85-87). More recent data from pediatric clinical trials, based on a protracted schedule that was developed in xenografts, showed a response rate of up to $60 \%$. As CPT is an S-phase poison, the pediatric studies emphasized the importance of scheduling of dosage and systemic exposure for the effectiveness of CPT and its analogs (88).

Both active and passive transport have been implicated in the intestinal uptake and efflux of CPT (89). A human ovarian cancer cell line IGROV1 was treated with topotecan and selected for resistance to the drug. It was found that the resistance was caused by an increased efflux of topotecan through the overexpression of the $\mathrm{ABC}$ transporter family member, ABCG2 (BCRP) (90). Moreover, mice lacking a functional ABCG2 have an increased oral bioavailability of topotecan and also patients treated with topotecan and GF120918, a known inhibitor of ABCG2, demonstrated an increase in drug bioavailability from $40 \%$ to $97 \%(91)$. In yeast, the transcription factor, PDR 1 , regulates the expression of various gene products including the ATP-binding cassette transmembrane transport proteins PDR5, YOR1 and SNQ2. A dominant mutation in PDR 1 was found in a screen for mutants that resulted in CPT resistance. While the PDR1 $\mathrm{T}^{879} \mathrm{M}$ mutation seems to increase the transcription of its downstream effectors, deletion of PDR 1 or its downstream target $S N Q 2$, increase CPT sensitivity, emphasizing the role of the pleiotropic drug resistance network in CPT toxicity. (92).

Cellular metabolism has a significant effect on the conversion of the prodrug Irinotecan (CPT-11) to its active form SN-38. Carboxylesterases are responsible for this conversion and an increase in the protein levels of these enzymes correlate with increased cellular sensitivity to CPT-11 (93). 
Top1 point mutations impacting the efficacy of CPT have been identified in both CPT-resistant tumor cell lines and yeast cells. As of yet, there has only been one report of a clinical sample that contained a mutation in TOP1. A patient with non-small cell lung cancer was preoperatively treated with Irinotecan (CPT-11) and cisplatin, but did not respond to the chemotherapy. It was found that there was a missense mutation in TOPI that resulted in a stop codon at position 736 and in case of a read through also had a glycine to serine substitution at position 737 (94).

Mutations in the highly conserved region preceding the active site Tyr cause CPT resistance through different mechanisms (95). For the top vac mutant enzyme, residues $\mathrm{Ile}^{725}$ and $\mathrm{Asn}^{726}$ in yeast Top1 were mutated to Arg and Ala, respectively, to match the corresponding residues of the vaccinia virus Top1. Even though the specific activity was comparable to wild-type Top1, the mutants were resistant to CPT in vivo, indicating that resistance is caused by either a decrease in drug binding or the formation of the ternary complex. Similar results were found by mutating the corresponding residues in human Top1. Another resistance mechanism was uncovered with the yeast $\operatorname{top}_{1} 1 \mathrm{~N}^{726} \mathrm{~L}$ mutant. It resulted in a decrease in catalytic activity (95). A screen that identified intragenic suppressors of the toxicity caused by the Top $1 \mathrm{~T}^{722} \mathrm{~A}$ mutant, a CPT mimetic, also found that all suppressors identified abolished catalytic activity. Biochemical analyses of some of these mutants suggest that this was mediated by a reduction in affinity for DNA (96). This mechanism of resistance is further supported by fact that several mammalian CPTresistant cell lines have been reported to have a reduction in Top1 catalytic activity (69, 97, 98).

Six mutations generating a CPT resistant phenotype $\left(\mathrm{Phe}^{361}, \mathrm{Gly}^{363}, \mathrm{Arg}^{364}\right.$, 
$\mathrm{Glu}^{418}, \mathrm{Gly}^{503}$ and $\mathrm{Asp}^{533}$ ) have been mapped to the "lips" region of human Top1. The lips are two loops that connect the cap with bottom lobe of the protein. Of these six mutations, only one has a direct protein-drug interaction namely, $\mathrm{Asp}^{533}$ in human Top1 (85). The crystal structure of the Top1-DNA-TPT ternary complex does not reveal the mechanism of resistance for the other mutations in this region, but it has been proposed that some of these residues might assist in forming a pocket for drug binding. This structure also shows that the Asn, adjacent to the catalytic Tyr, forms a water-mediated hydrogen bond with the drug (85), elucidating the mechanism underlying the resistance of the yeast top $1 N^{726} S$ and the related human top $1 N^{722} S$ mutant (99).

Reconstituted Top1 enzymes, either with or without the linker domain, are resistant to $\mathrm{CPT}$ in plasmid relaxation assays, suggesting that a functional linker is necessary for optimal CPT sensitivity (61). This was also demonstrated by a mutant Top1 enzyme that contained an $\mathrm{Ala}^{653}$ to Pro substitution located in the region of the linker domain that extends away from the core (62). Molecular dynamic analysis of this mutant protein displayed an alteration in the flexibility of this domain and in vivo studies showed that the cells were resistant to CPT (62). Both the reconstituted enzymes and linker domain mutant had an increased rate of religation, presumably decreasing the period for drug binding.

Interaction of Top1 with its protein partners may also dictate cellular sensitivity to CPT. The Top1-binding protein, nucleolin, recruits Top1 to the nucleolus (33) and deletion of the yeast nucleolin orthologue, NSR1, is associated with relocalization of Top1 from the nucleolus to a more diffuse nuclear distribution. These cells are also resistant to CPT. Top1 is known to move rapidly from the nucleolus to the nucleus after 
cellular exposure to CPT. Therefore, the loss of nucleolar localization may result in a decrease in the overall amount of Top1 associated with DNA and thus reduces the effect of CPT (100).

\subsection{REPAIR OF TOP1-MEDIATED DNA DAMAGE}

The normally transient Top1 covalent complexes can be converted into irreversible damage through various mechanisms. For example, when Top1 cleaves at or near a variety of DNA lesions, such as uracil mismatches, nicks, gaps or base damage caused by oxidative stress $(101,102)$, or the cleavage/religation equilibrium is skewed towards cleavage, there is an increase in stabilization of covalent complexes. Alteration in the equilibrium can be accomplished by either mutations in Top1 that increase the rate

of cleavage $\left(\right.$ Top $\left.1 \mathrm{~N}^{726} \mathrm{H}\right)$ or decrease in the rate of religation $\left(\mathrm{Top} 1 \mathrm{~T}^{722} \mathrm{~A}\right)(103)$ or by blocking religation by intercalation of drugs such as CPT. These lesions are toxic to actively replicating cells by a mechanism that involves collision of replication forks with the covalent complexes or as more recent data suggest, the accumulation of positive supercoils in front of the replication fork. Single molecule experiments demonstrated that CPT preferentially prevents the unwinding of positive supercoils which leads to the positive supercoil accumulation (104).

When the replication fork collides with the stabilized covalent complex, the extension of the leading strand is blocked at the 5'-end of the cleaved DNA template resulting in stalling of the replication fork and generation of single and double strand DNA breaks. These collisions trigger S-phase-specific cell death and cell cycle arrest in 
the G2-phase $(84,105)$. Studies in yeast and mammalian cells have implicated various biological pathways in the repair of these Top1-DNA lesions.

Treatment of human colon carcinoma cells (HCT116) with CPT induces phosphorylation of H2AX (phosphorylated form is called $\gamma \mathrm{H} 2 \mathrm{AX}$ ), an early event in the repair cascade of DNA double strand breaks. The phosphorylation is Top1 dependent as Top1-deleted mouse leukemia cells failed to induce $\gamma \mathrm{H} 2 \mathrm{AX}$. H2AX knockout cells are also hypersensitive to $\mathrm{CPT}$ indicating the phosphorylation of $\mathrm{H} 2 \mathrm{AX}$ is important for repair of Top1-mediated damage (106). A role for DNA double strand break repair pathway is also supported by the observation that yeast cells deleted for proteins involved in homologous recombination (main pathway for repair of DNA double strand breaks in yeast), such as $\operatorname{Rad50,} \operatorname{Rad} 51, \operatorname{Rad} 52$ and Sgs1 are hypersensitive to CPT $(55,66,107-$ 109).

Little is known about the processes that regulate the cellular responses to Top1induced DNA lesions. In yeast the Mec1 and Rad53 checkpoints modulate cell sensitivity to $\mathrm{CPT}$, consistent with studies in mammalian cells demonstrating the activation of the Sphase checkpoints, ATR/CHK1, in response to CPT treatment (110-112). Checkpoint activation prevents cells from entering mitosis with damaged DNA and provides additional time for DNA repair. Yeast cells deleted for Rad9, a DNA damage checkpoint protein that induces $\mathrm{G}_{2}$ arrest, also show enhanced CPT sensitivity (113).

For repair of Top1-DNA lesions, the protein must be removed, DNA breaks repaired and the replication fork restarted. The highly conserved protein Tyrosyl DNA phosphodiesterase (Tdp1) has been implicated in the repair of these covalent complexes as it has the ability to hydrolyze the 3'-phosphodiester bond between the catalytic 
tyrosine of Top1 and the 3'-end of the DNA. The removal of Top1 generates a 3'phosphate that is further processed by a 3'-phosphatase (114). Yeast cells devoid of Tdp1 are not hypersensitive to CPT, but when this deletion is combined with a deletion of $R A D 9$ (DNA damage checkpoint protein) there is a 10-fold increase in the cytotoxicity of CPT, indicating that Tdp1 has a role in the repair of Top1-induced damage, but also that there are Tdp1-independent repair pathways for these lesions (114). A mutation in the human $T D P 1$ gene, substitution of $\mathrm{His}^{493}$ for Arg located in the catalytic pocket, results in the hereditary neurological disease, spinocerebellar ataxia with axonal neuropathy (SCAN1). Cells derived from SCAN1 patients have reduced Tdp1 activity and are hypersensitive to CPT. Because hypersensitivity of these cells to CPT is not affected by the polymerase inhibitor, aphidicholin, it is postulated that Tdp1 is involved in the repair of Top1-induced lesions associated with transcription rather than replication (115).

Top1 can also be removed by the 3'-flap endonuclease complexes: XPF/ERCC1, Mre11/Rad50/Nbs1 and Mus81/Eme1.Their yeast orthologs are Rad1/Rad10, Mre11/Rad50/Xrs2 and Mus81/Mms4, respectively. Rad1/Rad10 and Tdp1 function in redundant pathways, while Mus81/Mms4 act in parallel $(116,117)$. The importance of Mus81 in repair of Top1-induced damage is highlighted by the fact that Mus81-deficient yeast cells are dramatically more sensitive to CPT (118). This protein has been shown to be involved in the processing of stalled and collapsed replication forks (119).

To determine events downstream of the ternary Top1-DNA-CPT complex, a genetic screen was performed to identify essential yeast genes that normally function to suppress cell sensitivity to CPT. As the pleiotropic drug resistance network affects yeast cell sensitivity to CPT, a Top1 mutant (Top $\left.1 \mathrm{~T}^{722} \mathrm{~A}\right)$ was used as the DNA damaging 
agent. This mutant mimics the action of CPT by decreasing the rate of enzyme-catalyzed religation and therefore overexpression of Top $1 \mathrm{~T}^{722} \mathrm{~A}$ induces cell death. The screen identified several mutants with defects in replication, amongst them Cdc45 and Dpb11. Dpb11 suppresses mutations in Pol $\varepsilon$ and possibly associates with the Pol $\varepsilon$ complex. This protein and its human homolog, TopBP, have also been shown to be components of the S-phase checkpoint. Cdc45 acts as a processivity factor for the replication helicase, is essential for the initiation of replication and is recruited to replication origins in an Sphase cyclin-Cdk-dependent manner (120). Both, Cdc45 and Dpb11 induce transient accumulation of Okazaki-sized fragments and enhance sensitivity to Top1 induced DNA damage, linking these processive DNA replication with cellular resistance (112).

\subsection{TOP1 LOCALIZATION}

Top1 is constitutively expressed throughout the cell cycle (121) and it is localized to the nucleus, being enriched in the nucleolus (122). Human Top1 has five nuclear localization signals (NLS) in its N-terminal domain, two of which seem to be mainly responsible for localization $(28,29,123)$. The $\mathrm{N}$-terminal domain anchors Top1 at distinct locations within nucleolus, concentrated in substructures identified as fibrillar centers. These nucleolar structures contain rDNA and a cluster of proteins termed the RNA Polymerse I holo-enzyme $(124,125)$. It is also the location of rDNA transcription (126). Top1 has been shown to co-localize (127) and co-purify with RNA Polymerase I (128) suggesting that it is part of this holo-enzyme. It was further demonstrated in $S$. cerevisiae that Top1 mutants exhibit an increase in rDNA recombination supporting a 
role for Top1 in maintaining integrity of the rDNA locus (129). The Top1 N-terminus mediates its interaction with the RNA Polymerase 1 holoenzyme at the fibrillar centers (20), as deletion of a portion of the N-terminus leads to the distribution of Top1 in the nucleolus in an amorphous and unstructured manner (127). Furthermore, studies from Drosophila melanogaster demonstrated that the N-terminus of Top1 targets it to transcriptionally active chromatin domains by interacting with transcription protein complexes $(33,130)$.

Rapid and reversible relocalization of Top1 to the non-nucleolar portion of the nucleus occurs after inhibition of RNA synthesis. This relocalization does not affect Top1 content or activity, and after the RNA synthesis inhibitor is removed, there is reversal of Top1 relocalization. This reversal occurs even in the presence of protein synthesis inhibitors, such as cyclohexamide (131). Top1 mobility is further revealed by FRAP (Fluorescence Recovery After Photobleaching) kinetics of GFP-tagged Top1. Even though the addition of a large polypeptide such as GFP can alter protein mobility, these experiments demonstrated that Top1 is fully mobile and continuously fluxes between nucleoli and the nucleoplasm, but with a slower mobility in the nucleoli. The catalytically inactive mutant, Top $1 \mathrm{Y}^{723} \mathrm{~F}$ has the same mobility in both compartments and is therefore evenly distributed throughout the nucleus (127).

Top1 is also lost from the fibrillar centers in response to treatment of cells with CPT. In reponse to this drug, Top1 rapidly relocalizes from the nucleolus to the nucleoplasm $(93,131)$. FRAP kinetics demonstrated that in response to CPT, the enzyme moves more slowly in the nucleoplasm than in the nucleoli. Therefore, it accumulates in 
the nucleoplasm and delocalizes from the nucleoli, although it is still freely exchanging between the two compartments (132).

Even though both CPT and Actinomycin D (RNA synthesis inhibitor) cause relocalization of Top1 away from the fibrillar centers, two separarate mechanisms appear to be involved. The localization response of Top1 to RNA synthesis inhibitors depends on the co-localization of the enzyme with the RNA Polymerase I complex, while the response to CPT requires catalytic activity (122).

Another nucleolar localized protein - nucleolin - interacts directly with the Nterminus of human Top1 and has been implicated in recruitment of Top1 to the nucleolus. Region 166-210 of human Top1 is sufficient for this binding (33). Loss of the NSR1 (nucleolin homolog in S.cerevisiae) caused a more diffused distribution of Top1 throughout the nucleus, underscoring the possible role for Nsr1 in concentrating Top1 in the nucleolar compartment (100). Contradictory results were found with imaging live HEK293 (human embryonic kidney) cells. Nucleolin did not co-localize with Top1 at the subnucleolar level. Moreover, a mutant lacking most of the N-terminal domain, but retaining the nucleolin binding site, does not show the distinct accumulation at the fibrillar centers as that was characteristic of the native protein (127). It was further found that the nucleolin binding region is neither sufficient nor necessary for nuclear and nucleolar localization (133).

CPT treatment also stimulates rapid modification of Top1 with small ubiquitinlike modifiers (SUMO) in response to high concentration of CPT and this modification correlates with nucleolar delocalization (134). In HELA cells, mutations in SUMO sites: $\mathrm{K}^{103} \mathrm{R}, \mathrm{K}^{117} \mathrm{R}$ and $\mathrm{K}^{153} \mathrm{R}$, enhances nucleolar accumulation of Top1 and abolishes 
nucleolar clearance in response to CPT (135). Although this suggested that the transport between nucleoli and nucleoplasm is triggered by attachment of SUMO, the results were challenged by more recent data obtained through life cell imaging of human embryonic kidney cells (HEK293) showing that silencing of the major SUMO sites does not affect relocalization after CPT treatment (122). Moreover, cells treated with self-poisoning Top1 mutant- Top $1 \mathrm{~T}^{722} \mathrm{~A}$ demonstrated no alteration in Top1 sumoylation relative to the wild-type enzyme (136). Due to contradictory results, it is not well understood what the role of sumoylation in Top1 localization is.

\subsection{TOP1 INTERACTORS}

Human Top1 has been shown to interact with the transcription initiation and elongation machinery, TATA Binding Protein (TBP), p53, nucleolin, SV40 large T antigen, RING finger/RS rich protein named topors, which function as a SUMO/Ub dual E3 ligase, and the spliceosomal protein, SF2/ASF. All of these interactions occur through the N-terminal Top1 domain, and only the binding motifs for nucleolin and SF2/ASF have been defined $(34,100,127,133,137)$.

Top1 is detected along transcriptonally active genes in Drosophila (138), colocalizes with the RNA Polymerase I (RPI) holoenzyme in immunohistochemical assays and co-purifies with RPI during biochemical purification of this enzyme $(128,139)$ indicating that Top1 has a role in rDNA transcription. These studies support earlier work in both budding and fission yeast demonstrating that rDNA transcription (highly transcribed region of the genome) is decreased in a top $1 \Delta$ strain (140). 


\subsection{POST-TRANSLATIONAL MODIFICATIONS OF TOP1}

Serine phosphorylation of Top1 from various organisms has been shown to increase the relaxation activity of this protein. Novikoff hepatoma cells are rapidly growing tumorigenic cells and Top1 isolated from these cells was phosphorylated (141). When treating extracts of Novikoff hepatoma cells with alkaline phosphatase, Top1 relaxation activity was reduced, while phosphorylation of the dephosphorylated protein with Type II Casein Kinase increased enzymatic activity $(142,143)$. Similar results were found with purified Top1 from Xenopus laevis (144), Chinese hamster (DC3F/9-OHE) and mouse leukemia cells (L1210) (145), where alkaline phosphatase completely abolished activity, but activity was regained after phosphorylation with Casein kinase II. Phosphoamino acid analysis showed that only serine residues are phosphorylated (144). In vitro phosphorylation generated three phopho-peptides, with one of these corresponding to the in vivo phosphorylation site found in Novikoff cells (141). In contrast to the increase in Top1 activity caused by serine activity phosphorylation, in vitro tyrosine phosphorylation of Top1 isolated from calf thymus decreased relaxation activity (146), suggesting that alteration in post-translational modifications (such a phosphorylation) is a possible mechanism for regulating Top1 activity.

Purified Top1 from calf thymus is poly (Adenosine-DiPhosphoribosylated) and enzymatic activity is concomitantly reduced $(147,148)$. Moreover, in vitro poly (ADPribosylation) also decreased the relaxation activity of Top1 (149). The possible role for this modification in repair of Top1-induced DNA damage is demonstrated by the fact that cell lines that are deficient in poly (ADP-ribosylation) are hypersensitive to CPT. This response is not solely for CPT induced damage, as the Top1-p53 molecular complex are 
poly (ADP-ribosylated) in response to irradiation (150), suggesting that this modification might play a more general role in the DNA damage response.

SUMO (Small ubiquitin-related modifiers) peptides are covalently attached to lysine residues located in a SUMO consensus sequence $\psi \mathrm{KXE}$, where $\psi$ is a large hydrophobic residue and $\mathrm{X}$ is any amino acid. Human Top1 has been shown to be sumoylated in reponse to high concentrations of CPT and it was suggested that this modification regulate Top1 localization between the nucleus and nucleolus (151). However, more recent evidence demonstrated that sumoylation of Top1 does not affect CPT-induced relocalization of the protein (122). Moreover, two independent yeast genetic screens to identify conditional hypersensitive mutants identified a mutation in the gene encoding the SUMO E2 conjugating protein, Ubc9 (136). When Pro ${ }^{123}$ was substituted for Leu ( $u b c 9-10)$, it caused a global decrease in sumoylation and enhanced cell sensitivity to CPT at the non-permissive temperature. In contrast, when Top1 SUMO consensus sites were altered, it did not recapitulate the sensitivity seen with the Ubc9 mutant, indicating that sumoylation of Top1 is not essential in protecting cells against CPT (136).

\subsection{STUDYING CPT SENSITIVITY IN YEAST}

The remarkable conservation of basic cell cycle machinery in combination with the multitude of genetic tools available, make the budding yeast, Saccharomyces cerevisiae, invaluable for studies of molecular pharmacology and cancer therapeutics (152). This unicellular organism was the first eukaryote for which the complete genome 
sequence was reported. The sequencing data revealed that the 12,068 kilobases of its haploid genome, packaged into 16 well-characterized chromosomes, defines 5885 potential protein coding genes $(152,153)$.

What makes this organism such a great model organism is its tractable genetics. Modifications such as gene disruption, gene marking, mutation or gene dosage effects, all of which are difficult to generate in higher eukaryotes, are relatively easy to create in yeast (152) . Therefore, with its well-defined genome and its facile genetics, budding yeast has become the organism of choice for genomic technology. This includes genome wide screens to elucidate cellular responses to various DNA damaging agents, such as CPT $(120,152)$ and ionizing radiation (154), determining protein-protein interaction through two-hybrid technology and high throughput screening to identify chemical inhibitors of heterologous proteins expressed in yeast (155).

Budding yeast has been invaluable in determining the catalytic mechanism and in vivo function of DNA topoisomerases (152). More specifically, using this system, Top1 was shown to be the sole target of the anticancer drugs camptothecin and indolocarbazole $(57,156)$ and it was further utilized to reveal cellular factors that modulate cell sensitivity to CPT and define mutations in Top1 that alters catalytic activity and confers drug resistance (26).

There are many advantages in using this single-celled organism for the study of DNA topoisomerase I. Unlike mammalian Top1, yeast Top1 is not essential and can be deleted without significant consequences. Therefore, top 1 alleles can be introduced and drug sensitivity and viability assayed without the presence of the wild-type enzyme. As homologous recombination is the major repair pathway in yeast, this characteristic can be 
utilized for the integration of DNA sequences into the genome. A single gene product can be manipulated in the context of an isogenic cell system and construction of such isogenic yeast strains are vital to establish the consequences of specific genetic alterations $(26,152)$.

Making use of this extraordinary model organism, we identified the first single residue in Top1 (Gly ${ }^{721}$ in yeast and $\mathrm{Gly}^{717}$ in human) that, when sunstituted for amino acids with a higher propensity to form $\alpha$-helices, increases CPT sensitivity. These substitutions include Asp, Asn, Glu, Gln, Leu and Ala. Using biochemical assays to uncouple the various steps in catalysis, we determined the mechanism for the increase in CPT sensitivity. As the altered residue is in a unique position to affect flexibility of the linker domain, and this domain of yeast and human Top1 differ in both length and amino acid sequence, but is believed to be structurally conserved, we determined what role the Gly to Asp substitution might have on the on the distinct linkers by generating chimeric enzymes. The results from the chimeric proteins and also differences in CPT sensitivities of the yeast and the human Gly mutants in vivo directed us to investigate potential binding partners of the linker domain and differences in the binding of these proteins to the yeast and human linker domains. 


\section{CHAPTER 2: SUBSTITUTION OF CONSERVED RESIDUES WITHIN THE ACTIVE SITE ALTER THE CLEAVAGE RELIGATION EQUILIBRIUM OF DNA TOPOISOMERASE I*}

\subsection{INTRODUCTION}

DNA topoisomerases catalyze changes in the linkage of DNA strands, playing critical roles in DNA replication, transcription, and recombination, as well as chromosome condensation and segregation $(3,4,7)$. Alterations in DNA topology are catalyzed in reactions characterized by the formation of a covalent enzyme-DNA intermediate. Eukaryotic DNA topoisomerase I (Top1) is a type IB enzyme that transiently cleaves a single strand of duplex DNA, while forming a tyrosyl linkage with the 3'-phosphoryl end of the cleaved DNA $(3,4,7)$. Rotation of the non-covalently held DNA around the nonscissile DNA strand allows for the relaxation of positive or negative supercoils. In a second transesterification reaction, the 5'-OH nucleophile attacks the phosphotyrosyl linkage to religate the DNA.

In eukaryotes, the nuclear type IB enzyme, encoded by the TOP I gene, is highly conserved in terms of amino acid sequence and catalytic mechanism $(3,4,7,26,157)$. The nuclear enzyme is also the cellular target of several antitumor agents, such as camptothecin (CPT) and indolocarbazole analogs $(84,105,158)$.

*William C.Colley, Mariè van der Merwe, John R. Vance, Alex Burgin, Jr., and MaryAnn Bjornsti Journal of Biological Chemistry 279 (52): 54069-54078, 2004 (103). Adapted by permission of publisher. 
The CPT analogs, Topotecan and SN-38 (the active metabolite of CPT-11), have shown remarkable antitumor activity against a broad range of pediatric and adult malignancies (73). These drugs target Top1 by reversibly stabilizing the covalent enzyme-DNA intermediate. During S-phase of the cell cycle, the collision of advancing replication forks with the ternary drug.Toplp·DNA complexes produces irreversible DNA lesions that trigger S-phase checkpoint activation, cell cycle arrest, and cell death $(3,84,105,152,157)$. Consistent with this cytotoxic mechanism, cells treated with aphidicolin to inhibit DNA replication are resistant to CPT (80). In the yeast Saccharomyces cerevisiae, strains deleted for specific DNA damage and replication checkpoint genes, such as RAD53, MEC1, TEL1, RAD9, RAD17, RAD24, and TOF1, exhibit enhanced sensitivity to CPT and other Top1 poisons $(62,113,154,159-161)$. Moreover, cells defective in processive DNA replication (because of alterations in Cdc45 or Dpb11) (120) or in maintaining replication fork stability (for example in cells deleted for $S G S 1)$ (118) are also hypersensitive to Top1-induced DNA lesions.

Structural determinations of human Topo-70, a 70-kDa C-terminal fragment of human DNA topoisomerase I, in covalent and noncovalent complexes with duplex DNA, revealed a circumferential binding of DNA by the Top1 protein clamp $(7,12,27)$. Using molecular modeling to design a reversible disulfide bond across the opposable lip domains that complete the clamp, we recently demonstrated that DNA rotation is inhibited within the locked Top1·clamp·DNA complex (41). Thus, some flexibility of Top1 domains is required for enzyme binding of DNA and the rotation of DNA strands necessary to effect changes in DNA topology. These studies further demonstrated that expression of the catalytically inactive Top $1 \mathrm{Y}^{723} \mathrm{~F}$-clamp in yeast cells with elevated 
levels of oxidized glutathione, also sufficed to induce cell lethality independent of covalent complex formation (41).

More subtle alterations in the DNA cleavage religation equilibrium catalyzed by Top1 have also been shown to be cytotoxic. For example, the introduction of an abasic site or a mismatch at the +1 position in the scissile DNA strand enhances the stabilization of the covalent complex (162). The structural distortions induced by incorporation of Ara-C at the +1 position of the complementary nonscissile strand also stabilize the covalent enzyme-DNA intermediate by reducing DNA religation (163). Mutation of conserved residues $\mathrm{N}$-terminal to the active site tyrosine in the yeast and human enzymes also induces Top1 poisoning $(99,113,156,164)$. Substitution of Ala for $\mathrm{Thr}^{722}$ in yeast Top1 (or Thr ${ }^{718}$ in human Top1) appears to mimic the action of CPT in stabilizing the covalent complex $(113,164)$. Based on the lack of detectable alterations in DNA binding or cleavage, a reduced rate of DNA religation by Top $1 \mathrm{~T}^{722} \mathrm{~A}$ was inferred (96), similar to that induced by CPT $(59,75)$. Recent structures of the binary Topo-70 DNA and ternary topotecan-Topo-70.DNA complexes indicate the formation of a hydrogen bond between the hydroxyl of $\mathrm{Thr}^{718}$ and the nonbridging oxygen of the +1 phosphodiester of the DNA (59). Thus, substitution of Ala at this position may alter the orientation of the 5'-OH nucleophile and impede DNA religation. A distinct self-poisoning mechanism was evidenced when $\mathrm{Asn}^{726}$ in yeast Top1 (or Asn ${ }^{722}$ in human Top1) was mutated to His. In this case, enhanced stabilization of the covalent enzyme-DNA complex was attributed to increased rates of DNA cleavage.

Other authors of this study demonstrated that the $\mathrm{T}^{722} \mathrm{~A}$ mutation directly inhibits Top1-catalyzed DNA religation, while Asn ${ }^{726}$ substitutions have diverse effects on 
enzyme sensitivity to CPT, Top1 binding to DNA, and enzyme-catalyzed DNA cleavage. Mutating $\mathrm{Asn}^{726}$ to His, Tyr, or Phe indicates that a phenolic or imidazole side chain enhanced the kinetics of DNA cleavage by Top1, whereas the CPT resistance of Top $1 \mathrm{~N}^{726} \mathrm{Q}$ suggests the orientation of the amide side chain is critical for effective drug binding. Further, the combination of the $\mathrm{T}^{722} \mathrm{~A}$ and $\mathrm{N}^{726} \mathrm{H}$ mutations in a single enzyme significantly potentiated the cytotoxic activity of the double mutant beyond that induced by co-expression of the individual mutant enzymes. Biochemical studies indicate that the independent alterations in DNA cleavage and religation, evident in the double mutant, each contribute to the lethal phenotype. My contribution to this work was the genetic studies that support the notion that the self-poisoning enzymes induce distinct cytotoxic lesions in vivo. These findings suggest that more effective Top1 poisoning may be achieved with a combination of therapeutics inducing distinct alterations in Top1 catalysis.

\subsection{EXPERIMENTAL PROCEDURES}

\subsubsection{Yeast strains and plasmids}

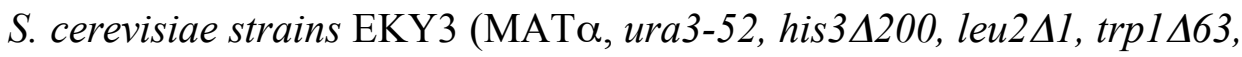
top1 $1:: T R P 1)$, MBY3 (EKY3, rad524::HIS3), MMY3 (EKY3, rad94::hisG), RRY71 (EKY3, dpb11-10), RRY72 (EKY3, cdc45-10) and RRY92 (EKY3, doa4-10) have been described $(96,112,113,120,165)$.

In plasmid YCpScTOP1, TOP1 is expressed from the constitutive TOP1 
promoter, (57). Plasmids YCpGAL1-top1 ${ }^{726} \mathrm{H}$, YCpGAL1-top1 $\mathrm{N}^{726} \mathrm{D}$, YCpSctop1T $\mathrm{T}^{722} \mathrm{~A}$, YCpGAL1-eTOP1·L were described $(96,99,113) . \mathrm{Asn}^{726}$ was mutated to Phe (top $1 \mathrm{~N}^{726} \mathrm{~F}$ ) with a QuikChange mutagenesis kit (Stratagene, La Jolla, CA) and oligonucleotide pairs: NFf (5'-

CACTGGGCACTTCCAAAATCTTTTATATAGACCCTAGAC-3') and NFr (5'GTCTAGGGTCTAtAtAAAAGAtTTtGGAAGTGCCCAGTG-3'). Mutant top1 sequences, excised with Bsu36I and XbaI, were ligated into YCpScTOP1 and YCpScTOP $1 \cdot \mathrm{L}$ vectors to yield $\mathrm{YCpSctop} 1 \mathrm{~N}^{726} \mathrm{H}, \mathrm{YCpSc} 1 \mathrm{~N}^{726} \mathrm{D}, \mathrm{YCpSctop} 1 \mathrm{~N}^{726} \mathrm{~F}$, and a $L E U 2$ marked $\mathrm{YcpSctop} 1 \cdot \mathrm{L}$ vector series.

Yeast cells were transformed by treatment with lithium acetate (166). URA3 and LEU2 plasmid transformants were maintained on synthetic complete media lacking uracil (SC-uracil) and leucine (SC-leucine), respectively, supplemented with 2\% dextrose or 2\% galactose.

In transformation assays, isogenic yeast strains EKY3 (top1 $\Delta$ ), MBY3 (top1 $\Delta$, rad524) and MMY3 (top14, rad94), RRY71 (top14, dpb11-10), RRY72 (top14, cdc4510) and RRY92 (top 14, doa4-10) were transformed with URA3 marked vectors (YCpScTop1, YCpSctop1 ${ }^{726} \mathrm{D}$, YCpSctop1 $\mathrm{N}^{726} \mathrm{H}$ or YCpSctop1T ${ }^{722} \mathrm{~A}$ ) or LEU2 marked vectors (YcpScTOP1·L, YCpSctop1N ${ }^{726} \mathrm{D} \cdot \mathrm{L}, \mathrm{YCpSctop} 1 \mathrm{~N}^{726} \mathrm{~F} \cdot \mathrm{L}$, YCpSctop $1 \mathrm{~N}^{726} \mathrm{H} \cdot \mathrm{L}$, or YCpSctop $1 \mathrm{~T}^{722} \mathrm{~A} \cdot \mathrm{L}$ ) and plated onto SC-uracil or SC-leucine, dextrose plates, respectively. Colony number and size were assessed after 3 days incubation at $30^{\circ} \mathrm{C}$. 


\subsubsection{Yeast cell viability}

To determine the viability of top $1 \triangle$ cells with the TOP 1 plasmids in temperature sensitive strains, exponential cultures of individual transformants were adjusted to an $\mathrm{OD}_{595}$ of 0.3 , serially 10 -fold diluted and 5 - $\mu 1$ aliquots were spotted onto selective plates. Cell viability was scored following incubation at 26 and $36{ }^{\circ} \mathrm{C}$.

\subsection{RESULTS}

Top1 Asn ${ }^{726}$ Mutants Induce Different Cellular Responses. The distinct mechanisms of stabilizing Top1 DNA complexes (increased DNA cleavage $\left(\mathrm{N}^{726} \mathrm{H}\right)$, decreased DNA religation $\left(\mathrm{T}^{722} \mathrm{~A}\right)$ and defects in DNA binding $\left.\left(\mathrm{N}^{726} \mathrm{D}\right)\right)$ raise the possibility that such alterations in enzyme catalysis may induce different DNA lesions and cellular responses. Toward this end, we determined if low constitutive expression of the mutant enzymes was tolerated in repair-proficient, DNA damage checkpoint competent cells. In contrast to wild-type Top1, high levels of Top $1 \mathrm{~N}^{726} \mathrm{H}$, Top $1 \mathrm{~N}^{726} \mathrm{D}$, and Top $1 \mathrm{~T}^{722} \mathrm{~A}$ expression achieved by the GAL1 promoter is sufficient to induce top $1 \triangle$ cell death in the absence of CPT $(96,99,113)$. However, low constitutive levels of Top $1 \mathrm{~T}^{722} \mathrm{~A}$, expressed from the yeast TOP 1 promoter are tolerated in top $1 \Delta$ cells, which are otherwise wild type for DNA repair and checkpoint pathways $(112,120)$. On the other hand, isogenic top $1 \Delta$ strains defective in homologous recombination (because of deletion of $R A D 52$ ) or the Rad9 DNA damage checkpoint (deletion of $R A D 9$ ) were unable to tolerate low levels of DNA damage induced by this topl mutant $(112,120)$ (Fig. 2.1). 


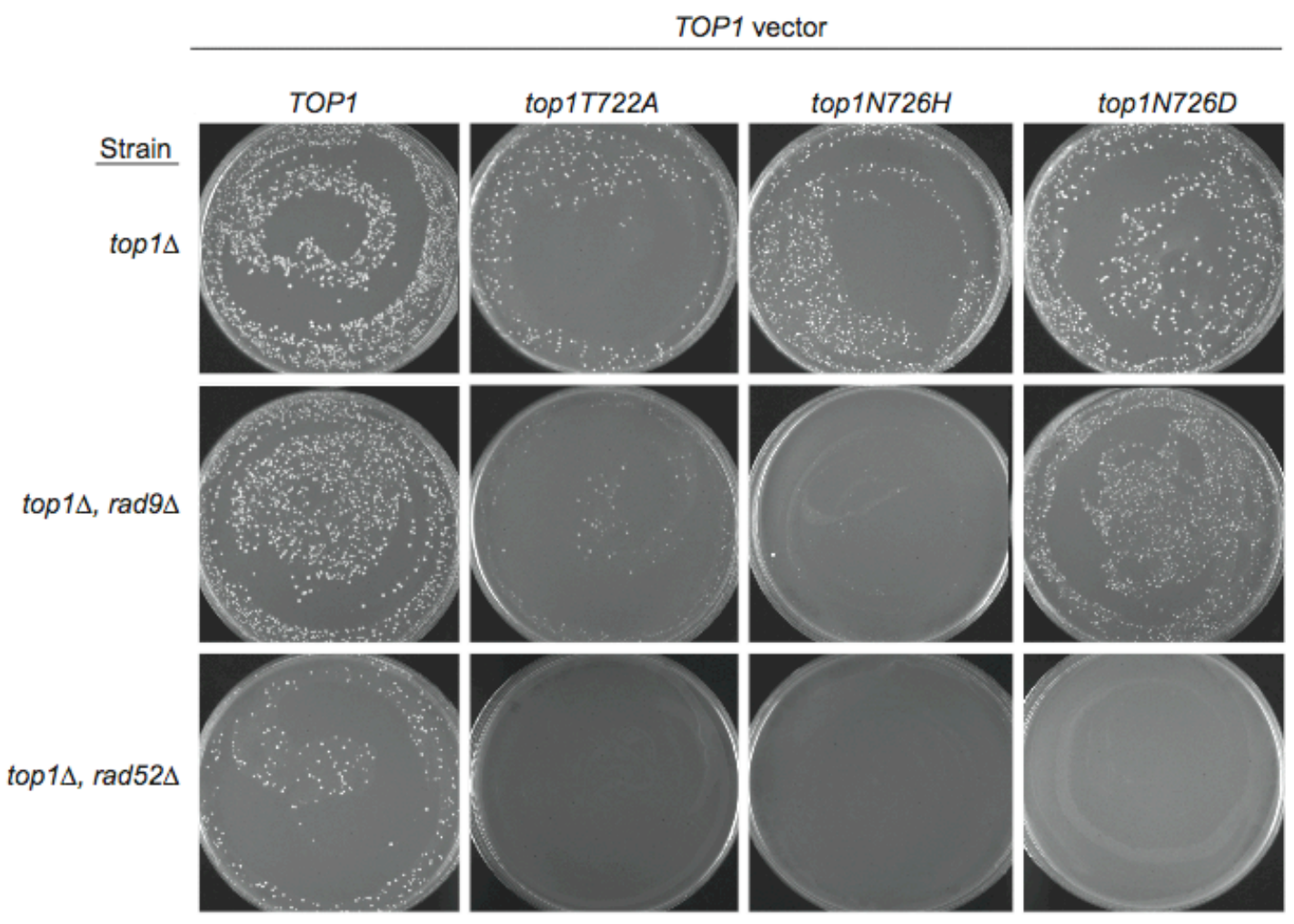

FIG. 2.1. Distinct cellular responses to lesions induced by self-poisoning Top1 enzymes are suggested in cells deleted for the Rad9 DNA damage checkpoint.

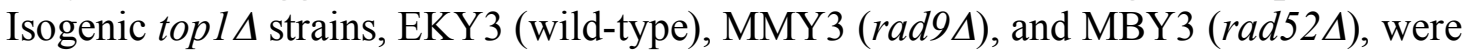
transformed with YcpScTOP1 vectors, where wild-type Top1 or the self-poisoning mutants top $1 N^{726} D\left(N^{726} D\right)$, top $1 N^{726} H\left(N^{726} H\right)$, and top $1 T^{722} A\left(T^{722} A\right)$, were constitutively expressed at low levels from the TOP 1 promoter. Following plating on SCuracil, dextrose media, the number and size of colonies formed by the plasmid transformed cells were determined after 3 days at $30{ }^{\circ} \mathrm{C}$. 
In plasmid transformation assays, cells defective in homologous recombination $(\operatorname{rad} 52 \Delta$ in Fig. 2.1 ; or $\operatorname{rad} 50 \Delta$, data not shown) were unable to tolerate low levels of Top $1 \mathrm{~T}^{722} \mathrm{~A}$, Top $1 \mathrm{~N}^{726} \mathrm{D}$, or Top $1 \mathrm{~N}^{726} \mathrm{H}$. However, cells deleted for the Rad9 DNA damage checkpoint exhibited variable sensitivity to the distinct self-poisoning enzymes. Isogenic $\mathrm{rad} 9 \Delta$ cells were inviable in the presence of $T o p 1 \mathrm{~N}^{726} \mathrm{H}$, exhibited a slow growth phenotype in the presence of Top $1 \mathrm{~T}^{722} \mathrm{~A}$ and were unaffected by expression of Top $1 \mathrm{~N}^{726} \mathrm{D}$. As similar levels of Top1 proteins were expressed in these isogenic top $1 \Delta$ strains, the distinct phenotypes were unlikely caused by differences in mutant enzyme levels. Rather, these results suggest two possibilities: the formation of distinct DNA lesions that trigger different checkpoint responses or a gradient of Top1·DNA damage in rank order Top $1 \mathrm{~N}^{726} \mathrm{H}>\operatorname{Top}_{1} \mathrm{~T}^{722} \mathrm{~A}>\mathrm{Top}_{1} \mathrm{~N}^{726} \mathrm{D}$.

To further investigate potential mechanism of Top1 poisoning, an additional substitution of $\mathrm{Asn}^{726}$ was engineered, and this mutant examined for its effect on cell viability. Based on the co-crystal structures of human Topo-70 $(59,85)$, the introduction of His at position $\mathrm{Asn}^{726}$ might enhance the formation of the phosphotyrosyl linkage by acting as a general base to accept the proton from the attacking hydroxyl of the active site tyrosine. Indeed, Top $1 \mathrm{~N}^{726} \mathrm{H}$ catalytic activity was unaltered over a wide range of $\mathrm{pH}$ values (data not shown), suggesting that His at this position is not positively charged. $\mathrm{Asn}^{726}$ was substituted with Phe $\left(\mathrm{N}^{726} \mathrm{~F}\right)$ to address issue of potential for stacking interactions, given the imidazole ring of His.

As depicted in Table 2.1, self-poisoning top $1 T^{722} A$, top $1 N^{726} D$, top $1 N^{726} H$, and top $1 N^{726} F$ mutants, constitutively expressed at low levels from the TOP 1 promoter on an $A R S / C E N$ vector, were used in a transformation assay similar to that described in Fig. 2.1. 
TABLE 2.1. Transformation efficiency of isogenic top1 yeast strains.

\begin{tabular}{llccc}
\hline & Wild-type & $c d c 45-10$ & dpb11-10 & doa4-10 \\
\hline TOP & +++ & +++ & +++ & +++ \\
top $1 \mathrm{~N}^{726} \mathrm{D}$ & +++ & - & - & ++ \\
top $1 \mathrm{~N}^{726} \mathrm{~F}$ & +++ & ++ & - & ++ \\
top $1 \mathrm{~N}^{726} \mathrm{H}$ & +++ & ++ & + & ++ \\
top1T $122 \mathrm{~A}$ & +++ & +++ & + & +++ \\
\hline
\end{tabular}

Isogenic top1 yeast strains (wild-type or tah mutants $c d c 45-10, d p b 11-10$ or doa4-10) were individually transformed with equal amounts of the indicated YCpScTOP1 vector that constitutively expresses low levels of Top1, Top $1 \mathrm{~N}^{726} \mathrm{D}$, Top $1 \mathrm{~N}^{726} \mathrm{~F}$, Top $1 \mathrm{~N}^{726} \mathrm{H}$, or Top $1 \mathrm{~T}^{722} \mathrm{~A}$. Following incubation at $26^{\circ} \mathrm{C}$, the number of transformed colonies was determined. In replicate experiments, +++ indicates 200-500 large colonies, ++ indicates 100-200 small colonies, + indicates 50-100 small colonies, and (-) refers to less than 10 transformants. 
However, the vectors were transformed into isogenic top $1 \Delta$ strains harboring different temperature sensitive tah strains (cdc45-10, dpb11-10, or doa4-10). The tah mutants were originally isolated in a yeast screen for enhanced sensitivity to the CPT mimetic, Top $1 \mathrm{~T}^{722} \mathrm{~A}$, at the nonpermissive temperature of $36^{\circ} \mathrm{C}(112,120)$. In comparison to wild-type cells, $c d c 45-10$ and $d p b 11-10$ strains exhibit defects in processive DNA replication, while doa4-10 cells are defective in ubiquitin-mediated proteolysis. As previously reported $(112,120,13,18)$, wild-type and tah mutant strains were readily transformed with the $T O P 1$-expressing vector and the resultant transformants were viable at 26 and $36^{\circ} \mathrm{C}$. However, the tah mutants (cdc45-10, $d p b 11$ 10, and doa4-10) were inviable at $36^{\circ} \mathrm{C}$ in the presence of low levels of $\mathrm{Top} 1 \mathrm{~T}^{722} \mathrm{~A}$ (Table 2.2 and Refs. $(112,120)$ ). This contrasts with the pattern of tah mutant sensitivity to the $\mathrm{Asn}^{726}$ mutants (Tables. 2.1 and 2.2). $d p b 11-10$ cells were unable to tolerate Top $1 \mathrm{~N}^{726} \mathrm{D}$ or Top $1 \mathrm{~N}^{726} \mathrm{~F}$ at any temperature, as no transformants were obtained (Fig. 2.2). With $c d c 45-10$ cells, $\operatorname{top} 1 N^{726} F$ transformants were viable at $26^{\circ} \mathrm{C}$; however, subsequent plating at $36{ }^{\circ} \mathrm{C}$ demonstrated a conditional lethal phenotype (Fig. 2.3). Since the $c d c 45-10$ and $d p b 11-10$ mutants exhibit a synthetic lethal interaction at $36^{\circ} \mathrm{C}$ in the absence of Top1 poisons $(112,120)$, a similar pattern of enhanced sensitivity to the selfpoisoning $\mathrm{N}^{726} \mathrm{D}, \mathrm{N}^{726} \mathrm{~F}$, and $\mathrm{N}^{726} \mathrm{H}$ mutants was not surprising. However, the temperature-sensitive lethality of $d o a 4-10$ cells expressing $T_{0 p} 1 \mathrm{~N}^{726} \mathrm{D}$ or $T o p 1 \mathrm{~T}^{722} \mathrm{~A}$ contrasts with the relative resistance to TopN ${ }^{726} \mathrm{~F}$ and $\mathrm{Top} 1 \mathrm{~N}^{726} \mathrm{H}$. When considered with the transformation assay of Fig. 2.1, these data support a model of distinct DNA lesions induced by top $1 N^{726} \mathrm{D}$ and $t o p N^{726} \mathrm{H}$ mutants and further indicate that different genetic backgrounds may be used to distinguish the cytotoxic action of distinct Top1 poisons. 
TABLE. 2.2. Growth at non-permissive temperature $\left(36^{\circ} \mathrm{C}\right)$.

\begin{tabular}{llllc}
\hline & Wild-type & $c d c 45-10$ & dpb11-10 & doa4-10 \\
\hline TOP & +++ & +++ & ++ & ++ \\
top1N726D & +++ & & & - \\
top1N726F & +++ & - & & ++ \\
top1N726H & +++ & - & - & ++ \\
top1T722A & +++ & - & - & - \\
\hline
\end{tabular}

Exponentially growing liquid cultures of individual transformants at $26^{\circ} \mathrm{C}(\mathrm{Fig} 2.1)$, were adjusted to an $\mathrm{OD}_{595}$ of 0.3 , serially 10 -fold diluted and spotted onto $\mathrm{SC}$-leucine media. Following incubation of duplicate plates at $26^{\circ} \mathrm{C}$ and $36^{\circ} \mathrm{C}$, cell growth was assessed. In shaded boxes, +++ indicates equivalent growth at both temperatures, while ++ indicates 10 -fold fewer colonies at $36^{\circ} \mathrm{C}$, relative to the same transformants at $26^{\circ} \mathrm{C}$. Although viable transformants were obtained at $26^{\circ} \mathrm{C}$, the cultured cells indicated by (-) failed to form colonies when plated at $36^{\circ} \mathrm{C}$. 


\subsection{DISCUSSION}

The self-poisoning activity of the Top1 mutant enzymes, Top $1 \mathrm{~N}^{726} \mathrm{H}$ and Top $1 \mathrm{~T}^{722} \mathrm{~A}(99,113,156)$, results from distinct alterations in enzyme-catalyzed DNA cleavage and religation. Based on the results of suicide substrate assays, the enhanced stabilization of Top $1 \mathrm{~T}^{722} \mathrm{~A} \cdot \mathrm{DNA}$ covalent complexes could not be attributed to alterations in DNA binding or rates of DNA cleavage (99). Rather, the data inferred a defect in DNA religation, similar to that induced by CPT. This model was supported by crystallographic data indicating the interaction of $\mathrm{Thr}^{718}$ (at the corresponding position in human Top1) with the nonbridging oxygen of the +1 phosphodiester of the DNA in a covalent complex formed by a 70-kDa C-terminal fragment of human Top1 and duplex DNA $(59,167)$. Substitution of this residue with alanine might alter the optimal alignment of the 5'-OH nucleophile in the transesterification reaction that restores the integrity of the phosphodiester backbone.

This work demonstrates that cell sensitivity to low, constitutive levels of distinct self-poisoning Top1 mutant enzymes varied dramatically in different genetic backgrounds. For example, cells deleted for the Rad9 DNA damage checkpoint were unable to tolerate low levels of Top $1 \mathrm{~N}^{726} \mathrm{H}$, exhibited a slow growth phenotype in the presence of Top $1 \mathrm{~T}^{722} \mathrm{~A}$ and were unaffected by expression of Top $1 \mathrm{~N}^{726} \mathrm{D}$ (Fig. 2.1). This contrasts with isogenic wild-type cells that easily tolerated expression of all three mutant enzymes. Based on steady state levels of covalent Top1·DNA complexes detected in DNA cleavage assays $(99,113)$, these phenotypes may reflect a gradient of increasing DNA damage induced be these enzymes, with $\mathrm{TopN}^{726} \mathrm{H}$ being the most potent selfpoisoning enzyme. However, several lines of evidence support an alternative model, 
whereby an increase in Top1-catalyzed DNA cleavage induces DNA lesions, some of which are intrinsically distinct from those induced by a decrease in Top1-catalyzed DNA religation.

First, the rate of cell killing induced by Top $1 \mathrm{~T}^{722} \mathrm{~A}$ or Top $1 \mathrm{~N}^{726} \mathrm{H}$ expression in top $1 \Delta$ cells was almost identical. (Data not shown) If Top $1 \mathrm{~N}^{726} \mathrm{H}$ were a more potent poison than Top $1 \mathrm{~T}^{722} \mathrm{~A}$, the kinetics of cell death would be faster. Second, as shown in Figs. 2.2 and 2.3, variable patterns of mutant Top $1 \mathrm{p}$ cytotoxicity were observed in a panel of isogenic tah mutant strains, originally isolated in a genetic screen for enhanced sensitivity to low levels of Top $1 \mathrm{~T}^{722} \mathrm{~A}$ at high temperature $(112,120)$. For example, cdc45-10 cells, which harbor a temperature sensitive hypomorphic allele of CDC45 (18), readily tolerate low levels of Top $1 \mathrm{~T}^{722} \mathrm{~A}$ expression at $26^{\circ} \mathrm{C}$ yet had decreased transformation efficiency with the same vector expressing Top $1 \mathrm{~N}^{726} \mathrm{H} . c d c 45-10$ cells exhibits a transient accumulation of Okazaki fragments upon shift to the nonpermissive temperature and are defective in processive DNA replication (120). In contrast, isogenic doa4-10 cells, which are wild-type for CDC45 but contains a nonsense mutation in DOA4 (112), are relatively resistant to Top $1 \mathrm{~N}^{726} \mathrm{H}$ and Top $1 \mathrm{~N}^{726} \mathrm{~F}$ expression even at $36^{\circ} \mathrm{C}$. Doa4 is a ubiquitin C-terminal hydrolase whose function is required to maintain free ubiquitin pools (112). Dosage suppression studies suggest that the enhanced sensitivity of doa4-10 cells to Top $1 \mathrm{~T}^{722} \mathrm{~A}$ is a consequence of specific alterations in the DNA damage checkpoint (112). Taken together, these data indicate that select genetic alterations in otherwise isogenic genetic backgrounds profoundly alter cell sensitivity to the cytotoxic action of different self-poisoning Top1 mutants. These findings further suggest that distinct mechanisms of Top1 poisoning, such as the enhanced rate of DNA cleavage by 
Top $1 \mathrm{~N}^{726} \mathrm{H}$ or Top $1 \mathrm{~N}^{726} \mathrm{~F}$ versus the defect in DNA religation evident with Top $1 \mathrm{~T}^{722} \mathrm{~A}$ or CPT induce distinct patterns of DNA lesions. In other words, how covalent complexes form is also an important determinant of cell viability. Whether this relates to the effective sensing and/or repair of such lesions in different genetic backgrounds has yet to be determined. 


\section{CHAPTER 3: SUBSTITUTIONS OF GLY ${ }^{721}$ ALTER YEAST DNA TOPOISOMERASE I ACTIVE SITE ARCHITECTURE AND ENZYME SENSITIVITY TO CAMPTOTHECIN}

\subsection{INTRODUCTION}

Eukaryotic DNA topoisomerase 1 (Top1) alters DNA topology by introducing a transient break in a single strand of the DNA duplex, thereby allowing strand rotation at the site of DNA scission to relieve superhelical tension $(3,4,7)$. The enzyme plays critical roles in processes such as replication, transcription, recombination and chromosomal condensation, with a reaction mechanism highly conserved from yeast to human. The active site tyrosine ( $\mathrm{Tyr}^{727}$ in yeast) acts as a nucleophile to cleave a single DNA strand, forming a covalent phosphotyrosyl linkage between the enzyme and the 3' end of the DNA. The free 5' $\mathrm{OH}$ end of the scissile strand then rotates about the uncleaved DNA strand in a manner dictated by torsional strain in the DNA. In a second transesterification reaction, the nucleophillic attack by the $5^{\prime} \mathrm{OH}$ resolves the covalent Top1-DNA intermediate, and the enzyme is liberated from the religated DNA.

DNA cleavage by Top1 is, at least in part, rate limiting, thus ensuring low steady state-levels of the covalent enzyme-DNA complex (168). However, increased concentrations of covalent complexes, either as a consequence of drug action, Top1 mutation, or the formation of DNA adducts, converts Top1 into a cellular poison $(84,99$, 101-103, 113, 152, 162, 164, 169-171). The camptothecin (CPT) class of chemotherapeutics reversibly stabilizes the covalent intermediate by intercalating into the 
protein-linked DNA nick and displacing the 5' $\mathrm{OH}$ end of the DNA to prevent religation (84, 152, 170). Moreover, recent studies demonstrate that CPT binding preferentially impedes Top1-catalyzed uncoiling of positively supercoiled DNA (172). Thus, during Sphase, either the ternary CPT-Top1-DNA complex itself, or the positive supercoils that accumulate between the replication machinery and the ternary complex, present obstacles to advancing replication forks and trigger the irreversible DNA lesions that induce cell death. Mutation of conserved residues within the enzyme active site may also alter the DNA cleavage/religation equilibrium catalyzed by Top1, leading to increased covalent complex formation and cell death $(99,113,164)$. For example, as with CPT treatment of wild-type Top1, substitution of Ala for $\mathrm{Thr}^{722}$ reduces the rate of enzyme catalyzed religation, while mutation of $\mathrm{Asn}^{726}$ to His enhances the rate of DNA cleavage (103). The same substitutions of the corresponding residues in human Top1 also produce "selfpoisoning" enzymes with similar effects on enzyme catalysis $(156,164)$.

Co-crystal structures of a $70 \mathrm{kDa}$ C-terminal fragment of human Top1 (Topo70) in noncovalent or covalent complexes with DNA revealed the unusual architecture of the monomeric enzyme $(12,27,59)$. Top1 forms a protein clamp around duplex DNA, where interaction of opposable "Lip" domains completes the circumscription of the tightly packed DNA by the protein core. We used molecular modeling to design a reversible disulfide bond between the lip domains, and demonstrated that locking the clamp prevents DNA rotation within the covalent Top1-DNA complex (41). Moreover, expression of a catalytically inactive Top $1 \mathrm{Y}^{723} \mathrm{~F}$-clamp proved toxic in the absence of DNA cleavage. The Top1 clamp consists of a conserved central core that includes all of the active site residues necessary for enzyme catalysis, except the active site $\operatorname{Tyr}^{723}$ (12, 
27, 28). A flexible linker physically connects the protein core with the conserved Cterminal active site tyrosine domain. The linker consists of a pair of $\alpha$-helices, arranged in an anti-parallel orientation, which extends out from the Top1 clamp and positions the C-terminal domain for enzyme catalysis. The poorly conserved N-terminal domain is missing in the Topo70 structures. Although these sequences have been implicated in DNA binding and in mediating protein-protein interactions, the N-terminus is dispensable for catalytic activity $(3,31,37,39)$.

Mutation of conserved residues in catalytically active yeast and human Top1 mutants has been reported to confer resistance to CPT [reviewed in $(26,85,170)]$. The majority of the mutated residues clusters along the Top1p-DNA interface or serve to alter the conformation of the protein clamp. Quantitative assays of drug binding are currently lacking; nevertheless, co-crystal structures of the CPT analog topotecan (TPT) bound to wild-type and mutant Topo70-DNA complexes suggest mutation-induced alterations in drug binding $(59,85)$.

In the covalent Topo70-DNA structures, the linker domain extends $\sim 56 \AA$ from the enzyme active site at an oblique angle to the DNA when TPT is bound. In the case of yeast Top1, residues corresponding to the linker domain are predicted to form a similar structure that would extend beyond $110 \AA$. In the absence of drug, the structure of these $\alpha$-helices in the covalent human Topo70-DNA complex was not resolved. Although present in Topo70, biochemical, genetic and molecular dynamic simulation data suggest that the flexibility of the linker in the absence of TPT would preclude structural determination $(59,61,62,173)$. Indeed, the physical connection and/or flexibility of the linker contribute to the CPT sensitivity of Top1. For instance, when Topo70 is 
reconstituted from separate polypeptides comprising the Top1 core and the linker/Cterminal domains, the enzyme is catalytically active yet exhibits reduced sensitivity to CPT (39). Within the N-terminal $\alpha$-helix of the linker, molecular dynamic simulations suggest mutation of $\mathrm{Ala}^{653}$ to $\operatorname{Pro}\left(\mathrm{A}^{653} \mathrm{P}\right)$ increases the flexibility of this coiled coil structure, to enhance the rate of DNA religation and Top1 resistance to CPT (62). More direct evidence for physical interactions between the Top1 linker and active site come from recent studies where the combination of the $\mathrm{A}^{653} \mathrm{P}$ mutation with the self-poisoning $\mathrm{T}^{722} \mathrm{~A}$ mutation suppressed the lethal phenotype of Top $1 \mathrm{~T}^{722} \mathrm{~A}$ (174). Taken together, these studies suggest alterations in the kinetics of Top1 catalyzed DNA cleavage/religation may be induced by changes in linker flexibility or orientation.

This model raises several questions: How are alterations in the physical linkage of the coiled coil transmitted to the active site to modulate enzyme catalyzed DNA cleavage/religation? How does this impact CPT binding to the Top1-DNA covalent complex? Implicit in these considerations is that a dynamic organization of the active site is either directly or indirectly affected by the orientation or flexibility of the linker domain. To begin to address these questions, we mutated conserved residues that bridge the C-terminus of the linker domain and the active site tyrosine domain, and asked what effect these substitutions had on yeast Top1 catalysis and CPT sensitivity. Our studies suggest that Gly ${ }^{721}$ in yeast Top1 constitutes a flexible hinge between the linker domain and a short $\alpha$-helix (delineated at the $\mathrm{C}$-terminus by the active site $\mathrm{Tyr}$ ) that modulates the geometry of the active site during enzyme catalysis, which directly impacts Top1 sensitivity to CPT. 


\subsection{EXPERIMENTAL PROCEDURES}

\subsubsection{Chemicals, yeast strains and plasmids}

Camptothecin purchased from Sigma was dissolved in DMSO and stored at -20

${ }^{\circ} \mathrm{C} .\left[\alpha-{ }^{32} \mathrm{P}\right]$ Cordycepin 5'-triphosphate was purchased from PerkinElmer Life Sciences (Boston, MA).

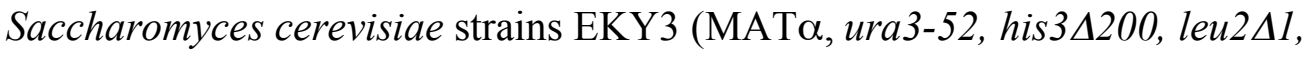
trp1 163 , top 1 $:: T R P 1)$ and MMY3 (EKY3, rad9 $::$ hisG) were described previously $(113,165)$. In plasmid YCpGAL1-eTOP1, wild-type Top1 containing an N-terminal Flag tag was expressed from the galactose-inducible $G A L 1$ promoter on an $A R S / C E N, U R A 3$ vector (113). Mutants top $1 G^{721} D$, top $1 G^{721} F$ or top $1 G^{721} V$, where residue $\mathrm{Gly}^{721}$ was mutated to Asp, Phe or Val, respectively, and $\operatorname{top} 1 L^{720} Q$, where $\mathrm{Leu}^{720}$ was mutated to Gln, were isolated from a pool of top 1 mutants generated by degenerate oligonucleotidedirected mutagenesis of residues flanking the active site $\mathrm{Tyr}^{727}$, as described in (113). Individual mutant sequences, excised in a Bsu36I-XbaI DNA fragment from YCpGAL1top1 vectors, were ligated into YCpGAL1-eTOP1 to generate YCpGAL1-etop $1 \mathrm{G}^{721} \mathrm{D}$, YCpGAL1-etop $1 \mathrm{G}^{721} \mathrm{~F}$, YCpGAL1-etop $1 \mathrm{G}^{721} \mathrm{~V}$ and YCpGAL1-etop $1 \mathrm{~L}^{720} \mathrm{Q}$. In plasmid pTP1-4, Gly ${ }^{721}$ was mutated to Leu, Asn, Glu, Gln or Ala $\left(\operatorname{top}_{1} G^{721} L\right.$, top $1 G^{721} N$, top $1 G^{721} E$, top $1 G^{721} Q$ or top $1 G^{721} A$ respectively) or Leu $^{720}$ to Glu (top $1 L^{720} E$ ) using a Quickchange mutagenesis kit (Stratagene, La Jolla, CA) and pairs of complementary oligonucleotides based on the following sequences: GL (5'CTCCCAGGTTTCACTGCTCACTTCCAAAATCAATT-3’); GN (5’'CAGGTTTCACTGAACACTTCCAAAATC-3'); GE (5'- 
CAGGTTTCACTGGAAACTTCCAAAATC-3'); GQ (5’-

GAAAACTCCCAGGTTTCACTGCAAACTTCCAAAATCAATTATAT-3'); GA (5' CTCCCAGGTTTCACTGGCCACTTCCAAAATCAATT-3') and LE (5'-

AAACTCCCAGGTTTCAGAGGGCACTTCCAAAATC-3'). Mutant sequences were excised with Bsu36I and Xba1 and ligated into YCpGAL1-eTOP1 to yield YCpGAL1etop $1 \mathrm{G}^{721} \mathrm{~L}$, YCpGAL1-etop $1 \mathrm{G}^{721} \mathrm{~N}$, YCpGAL1-etop $1 \mathrm{G}^{721} \mathrm{E}$, YCpGAL1-etop $1 \mathrm{G}^{721} \mathrm{Q}$, YCpGAL1-etop $1 \mathrm{G}^{721} \mathrm{~A}$ and YCpGAL1-etop1L ${ }^{720} \mathrm{E}$. In all cases, mutant sequences were confirmed by DNA sequencing. For ease of presentation, the e prefix, denoting the Nterminal Flag epitope in all of the constructs used in these studies, is dropped from the following discussions. Lithium acetate treated yeast cells were transformed with URA3marked plasmids (166) and individual transformants were maintained on synthetic complete media lacking uracil (SC-uracil) supplemented with $2 \%$ dextrose.

\subsubsection{Yeast cell sensitivity to CPT}

To examine cell sensitivity to CPT, exponential cultures of top $1 \Delta$ yeast cells transformed with the indicated YCpGAL1-top1 vector, were adjusted to an $A_{595}=0.3$, serially 10 -fold diluted and aliquots were spotted onto SC-uracil agar plates containing 25 mM HEPES ( $\mathrm{pH} 7.2$ ), $2 \%$ dextrose or galactose and the indicated concentration of CPT in a final $0.125 \%$ DMSO. Cell viability was scored following incubation at $30^{\circ} \mathrm{C}$. In more quantitative assays of cell viability, transformants grown in SC-uracil containing 25 mM HEPES, pH 7.2, (SC-uracil-HEPES) and dextrose, were diluted into SC-uracilHEPES supplemented with $2 \%$ raffinose. At an $A_{595}=0.3$, galactose ( $2 \%$ final) was added to induce Top1 expression, along with $50 \mu \mathrm{M}$ CPT in a final $0.43 \%$ DMSO or 
DMSO alone. At the times indicated, aliquots were serially diluted and plated onto SCuracil, dextrose plates and the number of viable cells forming colonies was determined following incubation at $30^{\circ} \mathrm{C}$.

\subsubsection{DNA topoisomerase I expression, purification and activity assays}

Extracts of galactose-induced cultures of EKY3 cells expressing plasmid encoded wild-type or mutant Top1 proteins were prepared essentially as described (95) except the glass beads were pre-chilled to $-20{ }^{\circ} \mathrm{C}$. Purified Top1 preparations were obtained by successive ammonium sulfate fractionations followed by phosphocellulose chromatography, as described (95). Top1 protein fractions were adjusted to a final concentration of $30 \%$ glycerol and stored at $-20{ }^{\circ} \mathrm{C}$.

To assess Top1 integrity and relative concentration, crude extracts, corrected for total protein concentration using a BioRad protein assay, or purified proteins were resolved in 4-12\% BisTris gels (Invitrogen), transferred onto activated polyvinylidene difluoride membranes (PerkinElmer Life Sciences), immunostained with the M2-Flag antibody (Sigma) and visualized by chemiluminescence. In the case of crude extracts, immunostaining with tubulin antibodies served as a loading control.

DNA topoisomerase I activity was assayed in plasmid DNA relaxation reaction as described $(99,103)$. Briefly, serial 10-fold dilutions of Top1 proteins, corrected for concentration, were incubated at $30{ }^{\circ} \mathrm{C}$ for 30 minutes with $0.3 \mu \mathrm{g}$ of negatively supercoiled plasmid pHC624 DNA in $20 \mathrm{mM}$ Tris (pH 7.5), $10 \mathrm{mM} \mathrm{MgCl}, 0.1 \mathrm{mM}$ EDTA, $50 \mu \mathrm{g} / \mathrm{ml}$ gelatin and $\mathrm{KCl}$ concentrations ranging from 50 to $200 \mathrm{mM}$. Reactions products were resolved in agarose gels and visualized by ethidium bromide staining. 


\subsubsection{DNA cleavage assays}

Wild-type and mutant enzyme sensitivity to CPT was determined in DNA cleavage assays as described $(99,103)$. A single $3{ }^{32}$ P-end-labeled DNA substrate was incubated with Top 1 proteins in $50 \mu 1$ reaction volumes containing Cleavage buffer [20 $\mathrm{mM}$ Tris (pH 7.5), $10 \mathrm{mM} \mathrm{MgCl}_{2}, 0.1 \mathrm{mM}$ EDTA, $50 \mathrm{mM} \mathrm{KCl}$, and $50 \mu \mathrm{g} / \mathrm{ml}$ gelatin] and the indicated concentration of CPT in a final 4\% (v/v) DMSO. After $10 \mathrm{~min}$ at $30{ }^{\circ} \mathrm{C}$, reactions were terminated with $1 \% \operatorname{SDS}$ at $75^{\circ} \mathrm{C}$, treated with proteinase $\mathrm{K}$, ethanolprecipitated and resolved in $8 \%$ polyacrylamide/7 $\mathrm{M}$ urea gels. Cleavage products were visualized with a PhosphorImager.

\subsubsection{Suicide cleavage reactions}

Oligonucleotide-based assays were used to uncouple Top1 catalyzed DNA cleavage and religation reactions. To assess the relative rates of DNA cleavage catalyzed by wild-type and mutant proteins, two substrates were used. First, as described in (103), a suicide substrate containing a truncated scissile strand with a high affinity cleavage site (5'-GATCTAAAAGACTT $\downarrow$ GG-3'), where $\downarrow$ indicates the phosphodiester bond cleaved

by Top1, was 5 '- end-labeled with $\left[\gamma_{-}{ }^{32} \mathrm{P}\right] \mathrm{ATP}$ and T4 polynucleotide kinase. Equimolar amounts of the labeled oligonucleotides were annealed to the non-scissile strand (5'GATCTTTTTTAAAAATTTTTCCGTCTTTTAGATC-3') in annealing buffer [10 mM Tris (pH 7.8), $100 \mathrm{mM} \mathrm{NaCl}, 1 \mathrm{mM}$ EDTA] by heating to $90{ }^{\circ} \mathrm{C}$ followed by cooling to room temperature over $3 \mathrm{~h}$. The DNA substrate ( $3 \mathrm{pmol} /$ reaction) was incubated with equal concentrations of Top 1 proteins in Cleavage buffer at room temperature. At the times indicated, $10 \mu \mathrm{l}$ aliquots were quenched with $0.5 \% \mathrm{SDS}$ at $75{ }^{\circ} \mathrm{C}$ to terminate the 
reaction. Following ethanol precipitation, the covalently linked protein was hydrolyzed with trypsin in the presence of $2 \mathrm{M}$ Urea and the labeled substrate and peptide-linked oligonucleotides were resolved in $16 \%$ polyacrylamide/7M Urea gels and visualized using a Phosphoimager. A second strategy was to monitor the liberation of a 6-mer downstream of the Top1 cleavage site, as described by Fertala et al. (99). Here, a slightly longer oligonucleotide containing the Top1 high affinity cleavage site (5'-

GATCTAAAAGACTT $\downarrow$ GGAAA-3') was 3'-end-labeled with [ $\left.{ }^{32} \mathrm{P}\right]$ cordycepin using terminal deoxynucleotide transferase. The labeled strand was annealed to equimolar amounts of same complementary strand described above. The DNA substrate (20 fmoles/reaction) was incubated with equal amounts of protein in Cleavage buffer at room temperature. At the times indicated, $10 \mu l$ aliquots were treated with $0.5 \%$ SDS at $75{ }^{\circ} \mathrm{C}$ and the reaction products were resolved in $20 \%$ polyacrylamide/7M Urea gels and visualized with a Phosphoimager.

\subsubsection{DNA religation assays}

Relative rates of Top1-catalyzed DNA religation were assessed using two oligonucleotide-based religation assays adapted from Colley et al., (103). In the postannealed religation substrate reactions, the same 5'-end labeled DNA suicide substrate described above, which contains the DNA scissile strand (5'-

GATCTAAAAGACTT $\downarrow$ GG-3'), was incubated with equal concentrations of Top1 proteins in Cleavage buffer at room temperature for 15 minutes to generate Top1-DNA cleavage complexes. Religation was then initiated $($ time $=0)$ by the addition of a 10 -fold excess of the religation oligonucleotide (5'-GGAAAAATTTTTAAAAAAGATC-3'). 
Alternatively, in reactions containing the pre-annealed religation substrate, the same DNA substrate was incubated with a 10 -fold excess of the religation strand for $15 \mathrm{~min}$ prior to the addition of Top1 proteins. In both assays, aliquots were processed as described above for the 5 'end labeled suicide cleavage reactions, except that when indicated, urea was omitted from the trypsin reaction to probe for alterations in active site architecture.

\subsection{RESULTS}

\subsubsection{Substitution of $\mathrm{Gly}^{721}$ and Leu ${ }^{720}$ alter Top1 sensitivity to CPT}

Within the active site pocket of yeast and human Top1, residues immediately Nterminal to the active site tyrosine are highly conserved (Fig. 3.1.E). Residues spanning $\mathrm{Thr}^{722}$ to the active site $\mathrm{Tyr}^{727}$ correspond to $\mathrm{Thr}^{718}$ to $\mathrm{Tyr}^{723}$ in human Top1, which form a short $\alpha$-helical structure highlighted in yellow in the co-crystal structures of human Topo70 covalently linked to DNA, either in the presence or absence of Topotecan (Fig. 3.1.A-D) $(59,175)$. Upon drug binding, however, this helical structure is extended to include conserved residues $\mathrm{Gly}^{717}$ and Leu ${ }^{716}\left(\mathrm{Gly}^{721}\right.$ and Leu ${ }^{720}$ in yeast Top1). This rather subtle transition in active site structure coincides with a more dramatic restriction in linker domain flexibility, such that the linker is now oriented at an oblique angle to the DNA. Additional studies suggest that linker domain flexibility as well as the physical linkage of the linker to both the active site $\mathrm{C}$-terminal domain and the protein clamp to which the C-terminus is abutted are critical determinants of enzyme sensitivity to CPT 

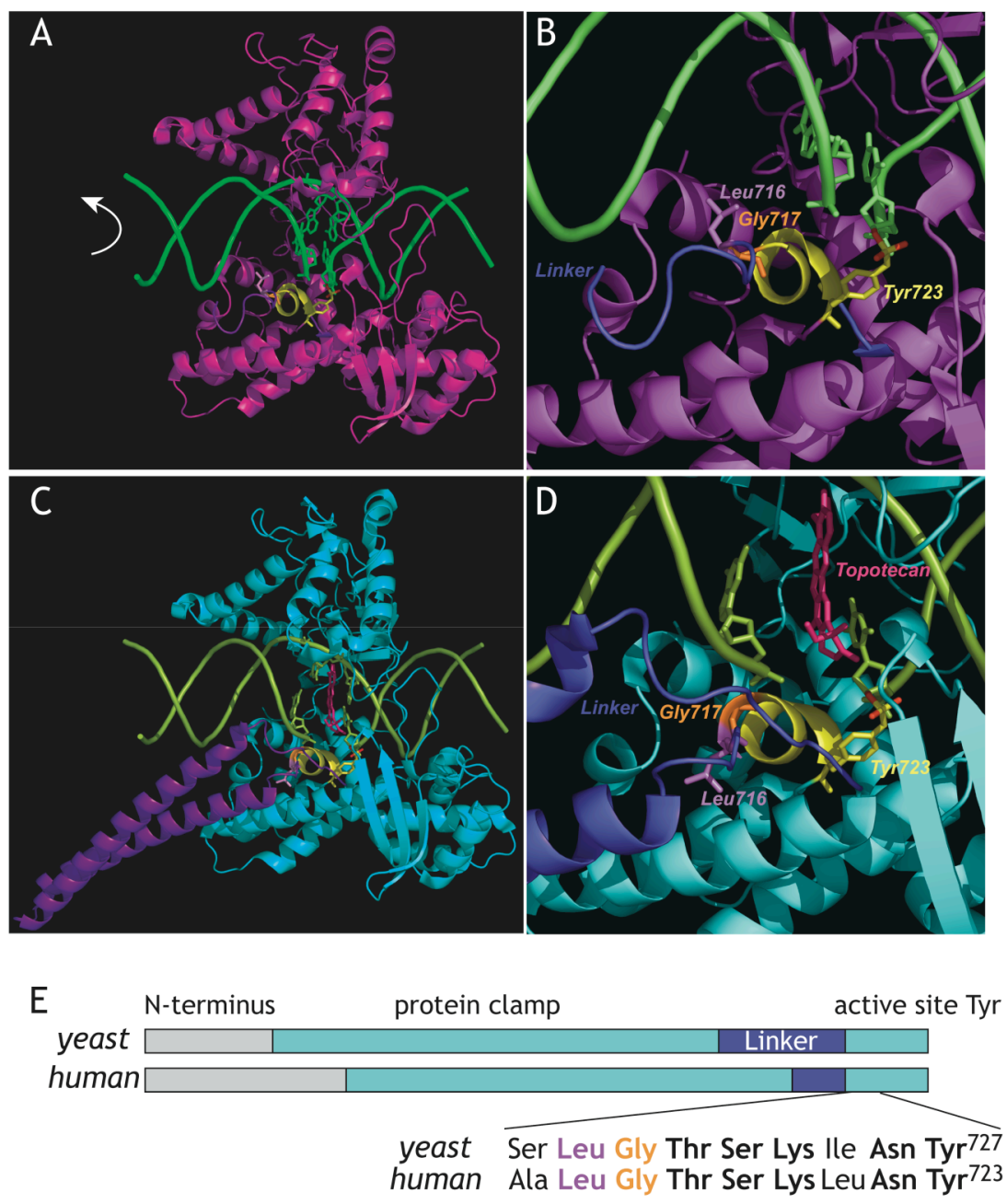

FIG. 3.1. Topotecan binding to the covalent Top1-DNA complex affects enzyme architecture and alters the geometry of the active site. Structures of the C-terminal 70 $\mathrm{kDa}$ of human Top1 (Topo70) covalently linked to duplex DNA in the absence $(A)$ or presence $(C)$ of topotecan (TPT) [PDB files $1 \mathrm{~K} 4 \mathrm{~S}$ and $1 \mathrm{~K} 4 \mathrm{~T}$ (59)]. $B$ and $D$, close up views of active site of Topo70-DNA and Topo70-DNA-TPT, respectively. A ribbon diagram of the polypeptide backbone spanning residues $\mathrm{Thr}^{718}$ to the active site $\mathrm{Tyr}^{723}$ is colored yellow. The covalent linkage between the 3' phosphoryl end of the DNA (green) and $\mathrm{Tyr}^{723}$ is shown in yellow and red solid bond representation. The base pairs spanning the Topo70-linked DNA nick are also shown and the conjugated ring structure of TPT is in magenta. $\mathrm{Gly}^{717}$ (corresponds to Gly ${ }^{721}$ in yeast Top1p) is in orange and $\mathrm{Leu}^{716}$ in lavender. $A, B$, residues spanning the $\mathrm{N}$ - and $\mathrm{C}$-terminal ends of the linker are highlighted in dark purple. $C, D$, the entire linker domain is shown in dark blue. All figures were made with PyMol (DeLano Scientific, San Carlos CA). E, Schematic representation of the domain structures of yeast and human Top1, with residues preceding the active site tyrosine. Color coding of the protein clamp, linker and C-terminal domains and conserved Leu and Gly residues correspond to that in $C$. 
$(61,62)$. Given the flexibility of Gly residues within a polypeptide chain, and the structural transitions apparent in the co-crystal structures with drug bound, we propose that $\mathrm{Gly}^{717}\left(\mathrm{Gly}^{721}\right.$ in yeast) constitutes a flexible hinge and that alterations in active site architecture at this junction would affect the orientation and flexibility of the linker domain. To address this hypothesis, we examined the effects of single amino acid substitutions of yeast $\mathrm{Gly}^{721}$ and the adjacent residue Leu ${ }^{720}$. These studies were initiated in yeast for several reasons: 1) the phenotypes of specific top 1 mutants could be assessed in the absence of wild-type Top1 (in yeast top $1 \Delta$ strains) (152); 2) any confounding effects of protein-protein interactions due to the expression of human Top1 in yeast could be avoided and 3) mutant enzyme activity could be assayed in different genetic backgrounds in otherwise isogenic strains.

Individual Gly ${ }^{721}$ and Leu ${ }^{720}$ substitutions were isolated from a pool of yeast Top1 active site mutants generated by degenerate oligonucleotide mutagenesis. Two hydrophobic substituents of $\mathrm{Gly}^{721}$, (Val in $\mathrm{G}^{721} \mathrm{~V}$ or Phe in $\mathrm{G}^{721} \mathrm{~F}$ ) led us to engineer a Leu mutation $\left(\mathrm{G}^{721} \mathrm{~L}\right)$ to expand the analysis of amino acid shape at this position. In contrast, the isolation of a $\mathrm{Leu}^{720}$ to $\mathrm{Gln}\left(\mathrm{L}^{720} \mathrm{Q}\right)$ mutation, prompted considerations of charge, so an acidic residue Glu was also engineered at this position $\left(\mathrm{L}^{720} \mathrm{E}\right)$. The effect of these single amino acid substitutions on cell sensitivity to CPT was assessed in top $1 \Delta$ cells induced to express the indicated mutant allele from the GAL1 promoter on an $A R S / C E N$ vector. As seen in Fig 3.2.A, substituting a basic or acidic residue for $\mathrm{Leu}^{720}$ abolished the CPT sensitivity of cells expressing Top $1 \mathrm{~L}^{720} \mathrm{Q}$ or Top $1 \mathrm{~L}^{720} \mathrm{E}$, respectively. These proteins were expressed at slightly higher levels that wild-type Top1; nevertheless, the specific activity of the two mutant enzymes was reduced by more than 100-fold (Fig. 
A
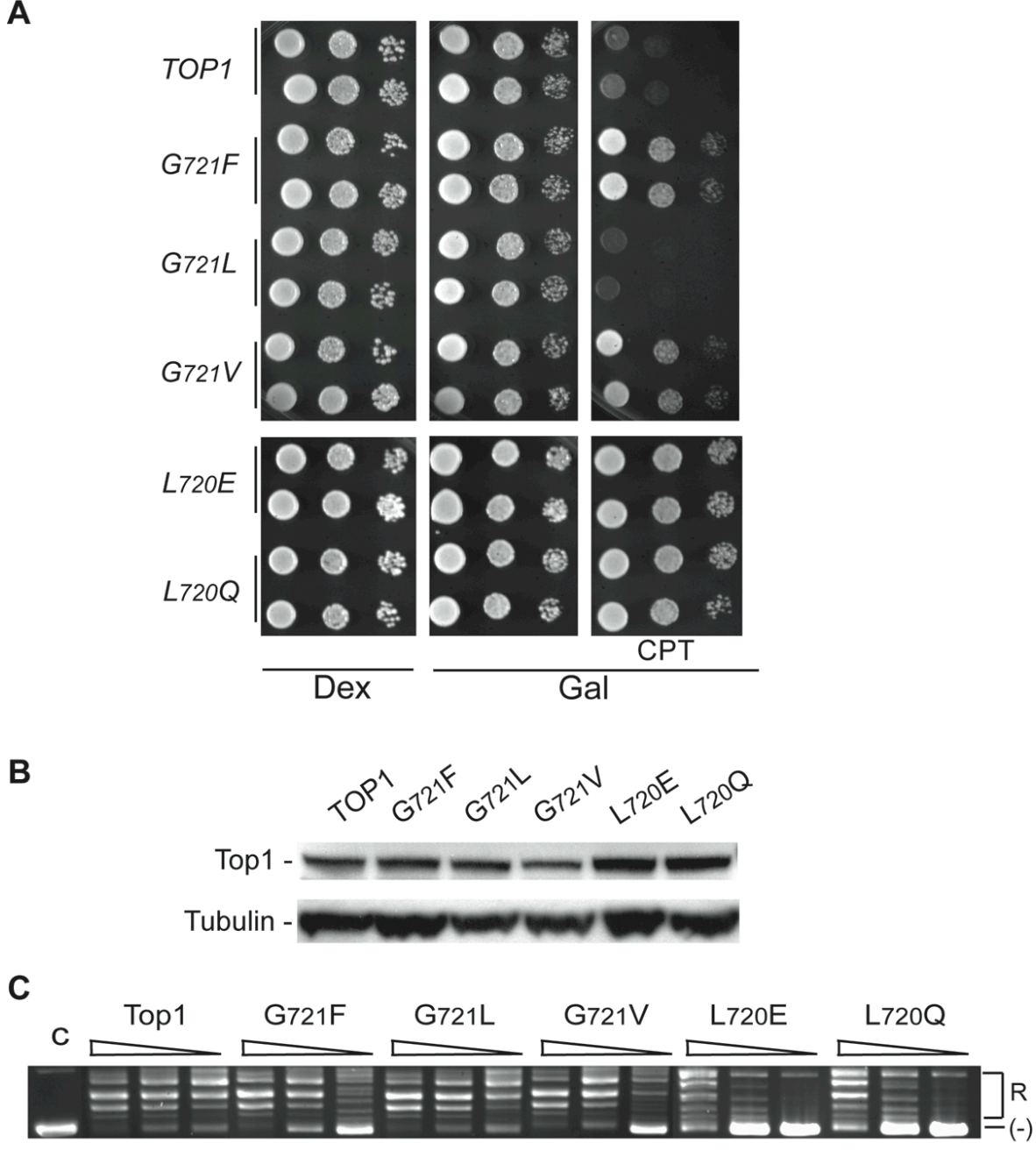

FIG. 3.2. Mutation of $\mathrm{Leu}^{720}$ or $\mathrm{Gly}^{721}$ in yeast Top1 induces CPT resistance in vivo. $A$, exponentially growing cultures of top $1 \Delta$ cells, transformed with the indicated YCpGAL1-top1 constructs, were adjusted to an $A_{595}=0.3$, serially 10 -fold diluted, and aliquots were spotted onto SC-uracil plates containing $25 \mathrm{mM}$ Hepes ( $\mathrm{pH} 7.2), 0.1 \%$ DMSO and either dextrose (Dex), galactose (Gal) or galactose plus $5 \mu \mathrm{g} / \mathrm{ml} \mathrm{CPT}$. The viability of two individual transformants was assessed following incubation at $30{ }^{\circ} \mathrm{C}$. B, levels of galactose-induced wild-type and mutant Top1 in crude cell extracts were determined in immunoblots with the M2-Flag antibody. Immunostaining with tubulin antibodies served as a loading control. $C$, the specific activity of wild-type and mutant Top1 enzymes was determined by incubating serial 10-fold dilutions of crude cell extracts (corrected for protein concentration) in plasmid DNA relaxation assays as described in Experimental Procedures. After $30 \mathrm{~min}$ at $30{ }^{\circ} \mathrm{C}$, the reaction products were resolved in a $1.2 \%$ agarose gel and visualized after ethidium bromide staining. The relative positions of relaxed (R) and supercoiled (-) DNA topoisomers are indicated. $\mathrm{C}$ is plasmid DNA control. 
3.2.B and C). These data suggest the CPT resistant phenotype of cells expressing the $\mathrm{L}^{720} \mathrm{Q}$ or $\mathrm{L}^{720} \mathrm{E}$ mutant results from a decrease in Top1 activity, rather than specific alterations in enzyme sensitivity to CPT. Indeed, homologous recombination defective rad52 2 , top $1 \Delta$ cells expressing Top $1 \mathrm{~L}^{720} \mathrm{E}$ were sensitive to 10 fold higher $\mathrm{CPT}$ concentrations than cells expressing wild-type Top1 (data not shown), indicating the formation of lower levels of CPT-induced Top $1 \mathrm{~L}^{720} \mathrm{E}$-DNA damage.

By contrast, substitution of hydrophobic residues for $\mathrm{Gly}^{721}$ had distinct effects on enzyme activity and cell sensitivity to $\mathrm{CPT}$. The $\mathrm{G}^{721} \mathrm{~L}$ mutation has no obvious affect on CPT-induced toxicity, while cells expressing Top $1 \mathrm{G}^{721} \mathrm{Vp}$ or Top $1 \mathrm{G}^{721} \mathrm{Fp}$ were CPT resistant (Fig. 3.2.A). All three mutant enzymes were expressed at levels comparable to wild-type Top1 (Fig. 3.2.B). The specific activity of Top $1 \mathrm{G}^{721} \mathrm{~L}$ was comparable to wildtype Top1, and the $\mathrm{G}^{721} \mathrm{~V}$ and $\mathrm{G}^{721} \mathrm{~F}$ mutants were slightly ( $\sim$-fold) less active in plasmid DNA relaxation assays (Fig. 3.2.C). However, expression of these mutants in a Rad9 DNA damage checkpoint defective ( $\operatorname{rad} 9 \Delta$ ) strain revealed that the $\mathrm{G}^{721} \mathrm{~L}$ mutant actually enhanced cell sensitivity to CPT, relative to cells expressing wild-type Top1 or the $\mathrm{G}^{721} \mathrm{~V}$ and $\mathrm{G}^{721} \mathrm{~F}$ mutants (Fig. 3.3.A).

The side chain of Val is branched at the $\beta$ carbon, while the $\gamma$ carbon branch of Leu is more distal to the polypeptide backbone. We then made additional substitutions of Gly $^{721}$, including Asp and Asn $\left(G^{721} \mathrm{D}\right.$ and $\mathrm{G}^{721} \mathrm{~N}$, respectively), to probe differences in charge independent of side chain geometry, and Glu and $\mathrm{Gln}\left(\mathrm{G}^{721} \mathrm{E}\right.$ and $\mathrm{G}^{721} \mathrm{Q}$, respectively), wherein the acidic and basic side chains are extended by a $\gamma-\delta$ carbon bond. As seen in Figs. 3.3.A and 3.4, substituting the acidic residues for $\mathrm{Gly}^{721}$ increased cell sensitivity to CPT by more than a factor of ten. However, as with the $\mathrm{G}^{721} \mathrm{~L}$ mutant, a 

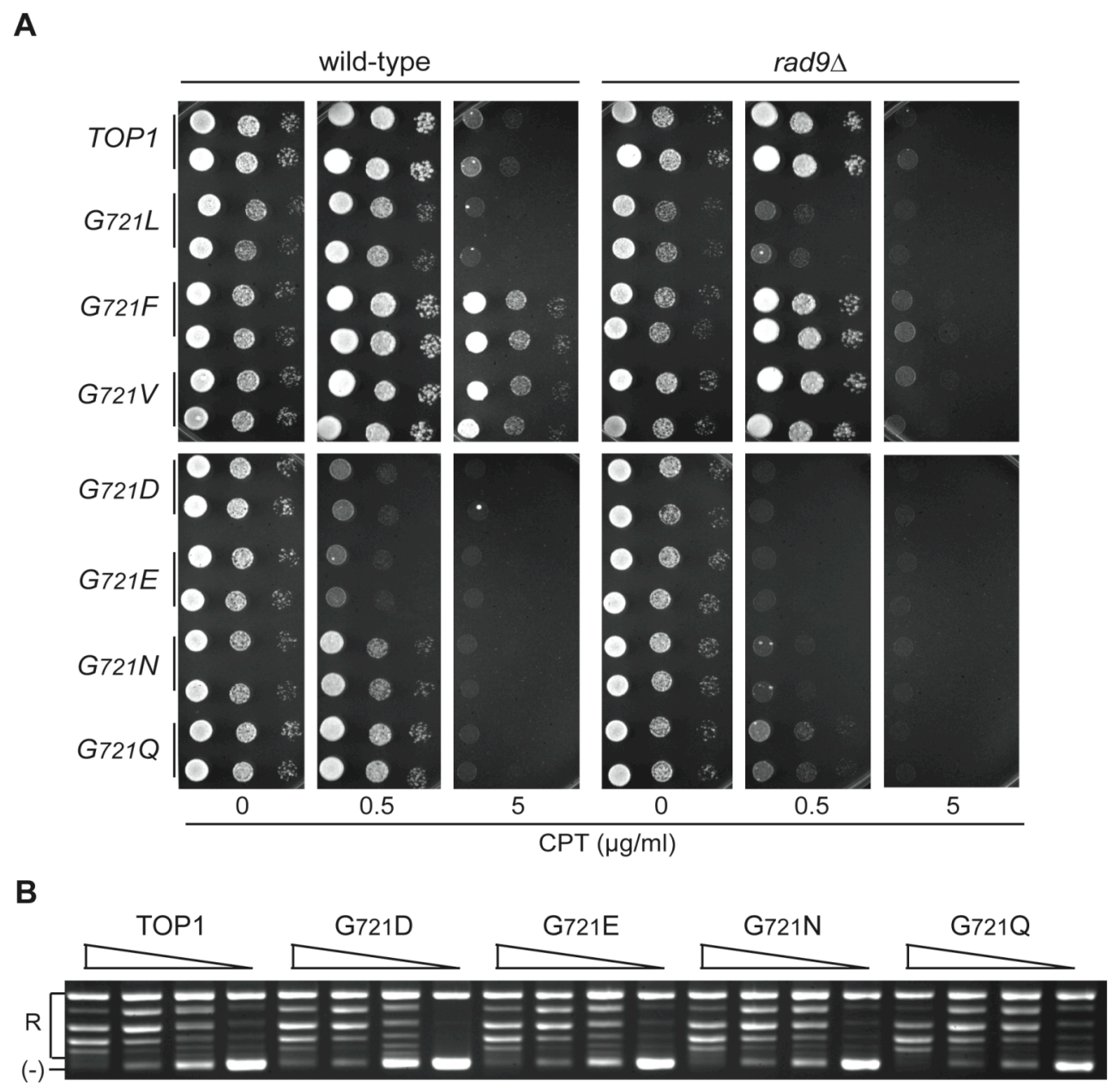

FIG. 3.3. Substitution of $\mathrm{Gly}^{721}$ with residues containing $\gamma$-carbon branched side chains enhance cell sensitivity to CPT. $A$, as in Fig. 3.2, exponential cultures of isogenic top $1 \Delta$ strains (wild-type for $R A D 9$ or rad9 4 ), transformed with the indicated YCpGAL1-top1 constructs, serially diluted and spotted onto SC-uracil plates containing $25 \mathrm{mM}$ Hepes ( $\mathrm{pH} 7.2$ ), 0.1\% DMSO, galactose and the indicated concentration of CPT. The viability of independent transformants was assessed following incubation. $B$, the specific activity of equal concentrations of purified Top1 and Gly ${ }^{721}$ mutant enzymes was assessed in plasmid DNA relaxation assays as described in Experimental procedures and Fig. 3.2 legend. The relative positions of relaxed (R) and supercoiled (-) DNA topoisomers are indicated. 


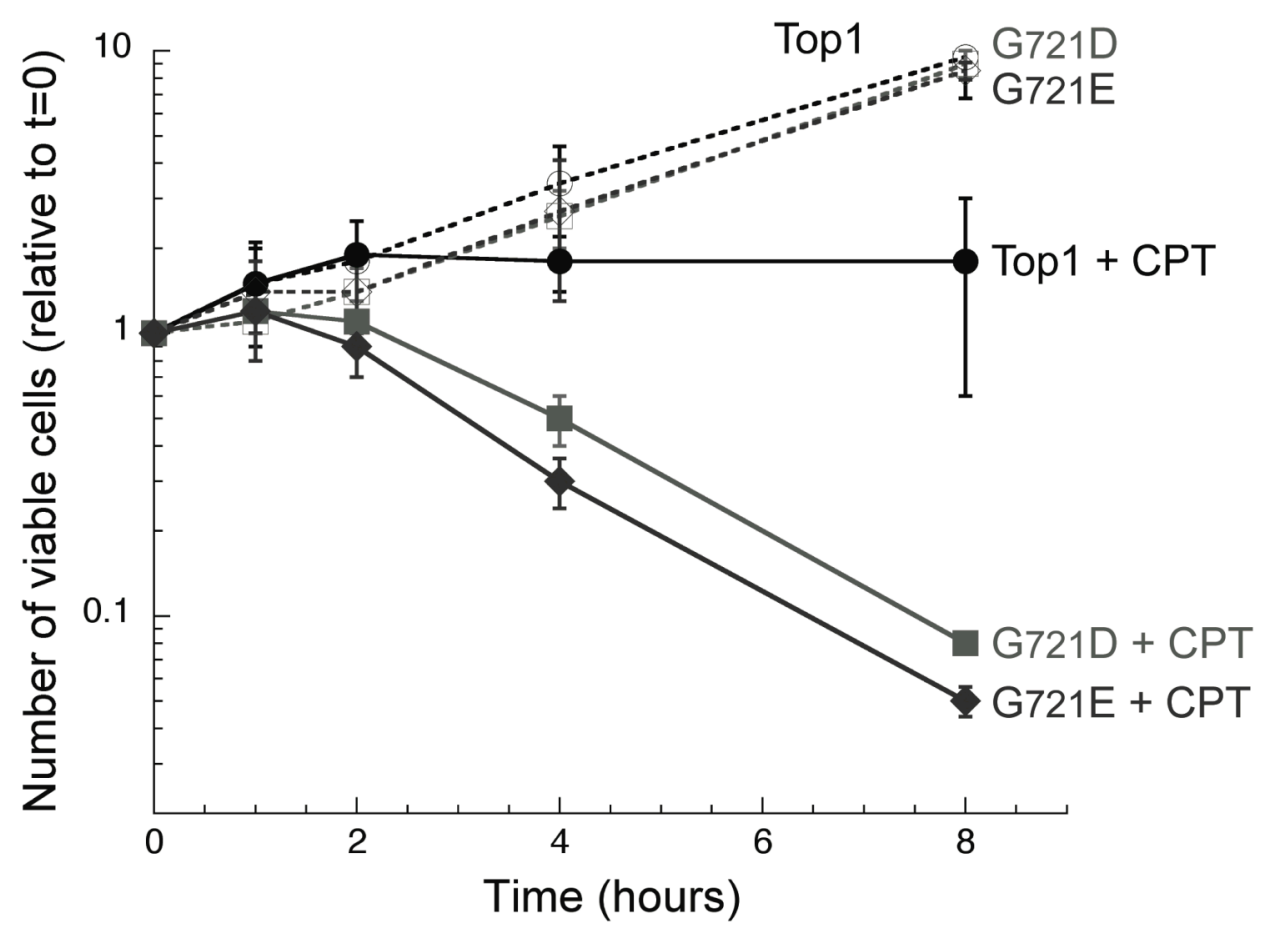

FIG. 3.4. Expression of Top $1 G^{721} D$ and $T o p 1 G^{721} E$ increases the cytotoxicity of CPT. At $t=0$, exponential cultures were treated with galactose, to induce expression of plasmid-encoded Top1, Top $1 \mathrm{G}^{721} \mathrm{D}$ or Top $1 \mathrm{G}^{721} \mathrm{E}, 0.43 \% \mathrm{DMSO}$ and, as indicated, 50 $\mu \mathrm{M}$ CPT. At the times indicated, aliquots were serially diluted and plated onto SC-uracil media containing dextrose. The number of viable cells forming colonies was assessed after $30^{\circ} \mathrm{C}$ for 3 days and plotted relative to the number obtained at $\mathrm{t}=0$. Results are an average of three independent experiments. 
significant increase in cell sensitivity to $\mathrm{CPT}$ as a result of $\mathrm{G}^{721} \mathrm{~N}$ or $\mathrm{G}^{721} \mathrm{Q}$ mutant

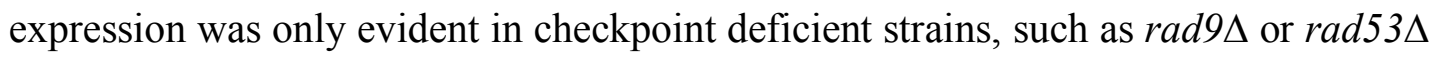
cells (Fig. 3.3.A and data not shown).

We previously described active site mutations, such as $\mathrm{T}^{722} \mathrm{~A}$ and $\mathrm{N}^{726} \mathrm{H}$, which enhance Top1 sensitivity to CPT in vitro $(99,113)$. However, these mutant enzymes induced cell lethality in the absence of CPT and exhibited other alterations in enzyme catalysis. In contrast, the Gly ${ }^{721}$ mutants that enhanced cell sensitivity to CPT, failed to induce growth defects in the absence of drug. In addition, as shown for Top $1 \mathrm{G}^{721} \mathrm{~L}$ (Fig. 3.2.B and $\mathrm{C}$ ), there were no detectable differences in the levels of wild-type and mutant Top1 proteins in galactose-induced cells and the specific activities of the purified $\mathrm{G}^{721} \mathrm{D}$, $\mathrm{G}^{721} \mathrm{E}, \mathrm{G}^{721} \mathrm{~N}$ and $\mathrm{G}^{721} \mathrm{Q}$ mutant proteins were indistinguishable from that of wild-type Top1 (Fig. 3.3.B).

\subsection{2. $\mathrm{Gly}^{721}$ mutations increase Top1 sensitivity to CPT in vitro}

We next asked if the increased CPT sensitivity of cells expressing the $\mathrm{G}^{721} \mathrm{D}$, $\mathrm{G}^{721} \mathrm{E}$ or $\mathrm{G}^{721} \mathrm{~N}$ mutants was a direct effect of increased CPT-Top1-DNA covalent complexes. First, the specific activity of these mutant enzymes exhibited the same salt optimum as wild-type Top1; similar patterns of activity and DNA topoisomer

distributions were observed for $\mathrm{G}^{721} \mathrm{D}, \mathrm{G}^{721} \mathrm{E}$ and $\mathrm{G}^{721} \mathrm{~N}$ mutant enzymes (Fig. 3.5. A and data not shown). Equal concentrations of the proteins were then incubated with a ${ }^{32} \mathrm{P}-$ single end-labeled DNA and increasing concentrations of CPT, to assess the intrinsic CPT sensitivity of each mutant enzyme relative to wild-type Top1. In such DNA cleavage assays (Fig. 3.5.B), all three mutants exhibited higher levels of drug-stabilized 
A
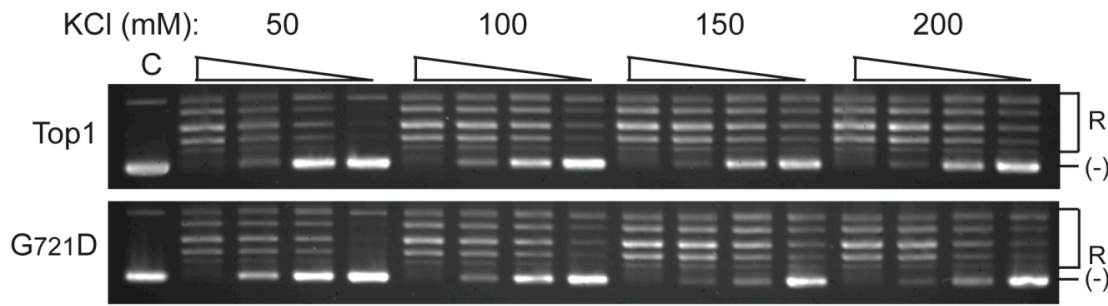

B

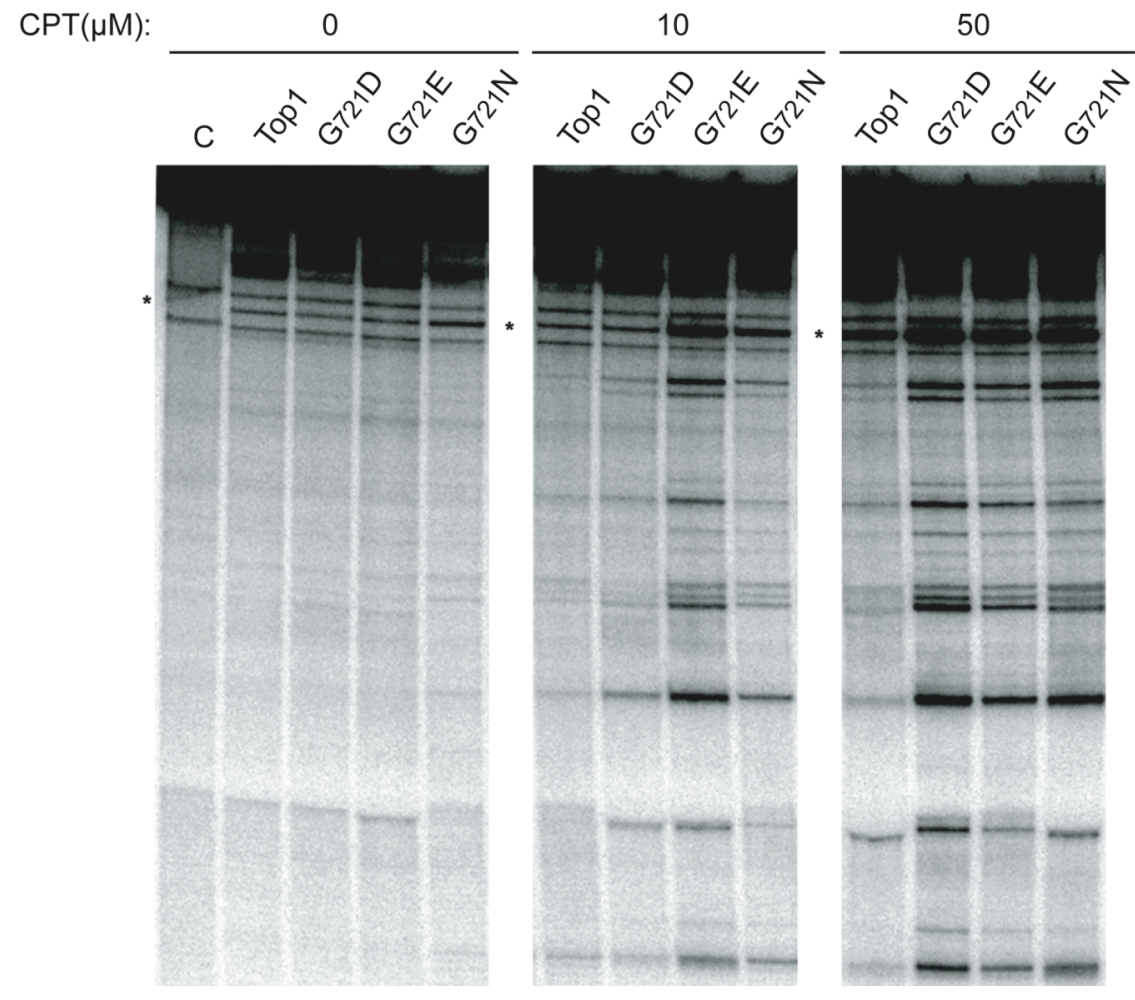

FIG. 3.5. Top $1 G^{721} D$, Top $1 G^{721} E$ and $T o p 1 G^{721} N$ exhibit increased levels of covalent complexes in the presence of CPT. $A$, equal concentrations of Top 1 and Top $1 \mathrm{G}^{721} \mathrm{D}$ were serially 10 -fold diluted and incubated in a plasmid DNA relaxation assay containing the indicated concentration of $\mathrm{KCl}$. After $30 \mathrm{~min}$ at $30{ }^{\circ} \mathrm{C}$, the reaction products resolved by agarose gel electrophoresis and visualized with ethidium bromide. The relative positions of relaxed (R) and supercoiled (-) DNA topoisomers are indicated. $B$, equal concentrations of Top 1 and mutant proteins were incubated with a single 3 , ${ }^{32} \mathrm{P}$-end labeled DNA substrate in a DNA cleavage reaction containing the indicated amount of CPT. After incubation for $10 \mathrm{~min}$ at $30{ }^{\circ} \mathrm{C}$, covalent complexes were trapped with SDS at $75{ }^{\circ} \mathrm{C}$ and treated with proteinase $\mathrm{K}$. The reaction products were resolved in $8 \%$ polyacrylamide/7M urea gels and visualized using a PhosphoImager. $\mathrm{C}$ is DNA alone and the asterisk $(*)$ indicates a high affinity Top1p cleavage site. 
Top1-DNA covalent complexes than that observed with wild-type Top1: the acidic substituent $\mathrm{Glu}\left(\mathrm{G}^{721} \mathrm{E}\right)$ conferred high levels of DNA cleavage across a wide range of CPT concentrations, while mutation to Asp $\left(G^{721} D\right)$ enhanced DNA cleavage to the greatest extent at higher drug concentrations. Indeed, these findings are consistent with the enhanced CPT sensitivity of cells expressing the $\mathrm{G}^{721} \mathrm{D}$ and $\mathrm{G}^{721} \mathrm{E}$ mutants (Figs. 3.3 and 3.4). Increased levels of covalent complexes were also evident with the $\mathrm{G}^{721} \mathrm{~N}$ mutant, but not at lower drug concentrations (as with $\mathrm{G}^{721} \mathrm{E}$ ) or to the same extent as $\mathrm{G}^{721} \mathrm{D}$ at higher concentrations. Together, these data suggest that the increased CPT sensitivity of cells expressing these mutants can be attributed to mutation-induced increases in drug poisoning of Top1.

\subsubsection{Replacing Gly ${ }^{721}$ with acidic residues affect Top1 cleavage of DNA and enzyme architecture in the region preceding the active site Tyr}

Top $1 \mathrm{G}^{721} \mathrm{D}$ and Top $1 \mathrm{G}^{721} \mathrm{E}$ did not exhibit altered steady state levels of Top1DNA covalent complexes in the absence of CPT, or specific enzyme activity in plasmid DNA relaxation assays, yet exhibited increased CPT sensitivity in vivo and in vitro (Figs. 3.4 and 3.5). We considered that changes in active site geometry induced by these substituents might enhance CPT binding and coincide with changes in linker domain flexibility. To address such dynamic interactions, we first used oligonucleotide-based DNA substrates to uncouple Top1 catalyzed DNA cleavage from religation.

As depicted in Fig. 3.6.A, Top1 cleavage of a high affinity site within a suicide DNA substrate liberates a 3' end labeled 6-mer and traps the covalent Top1-DNA 

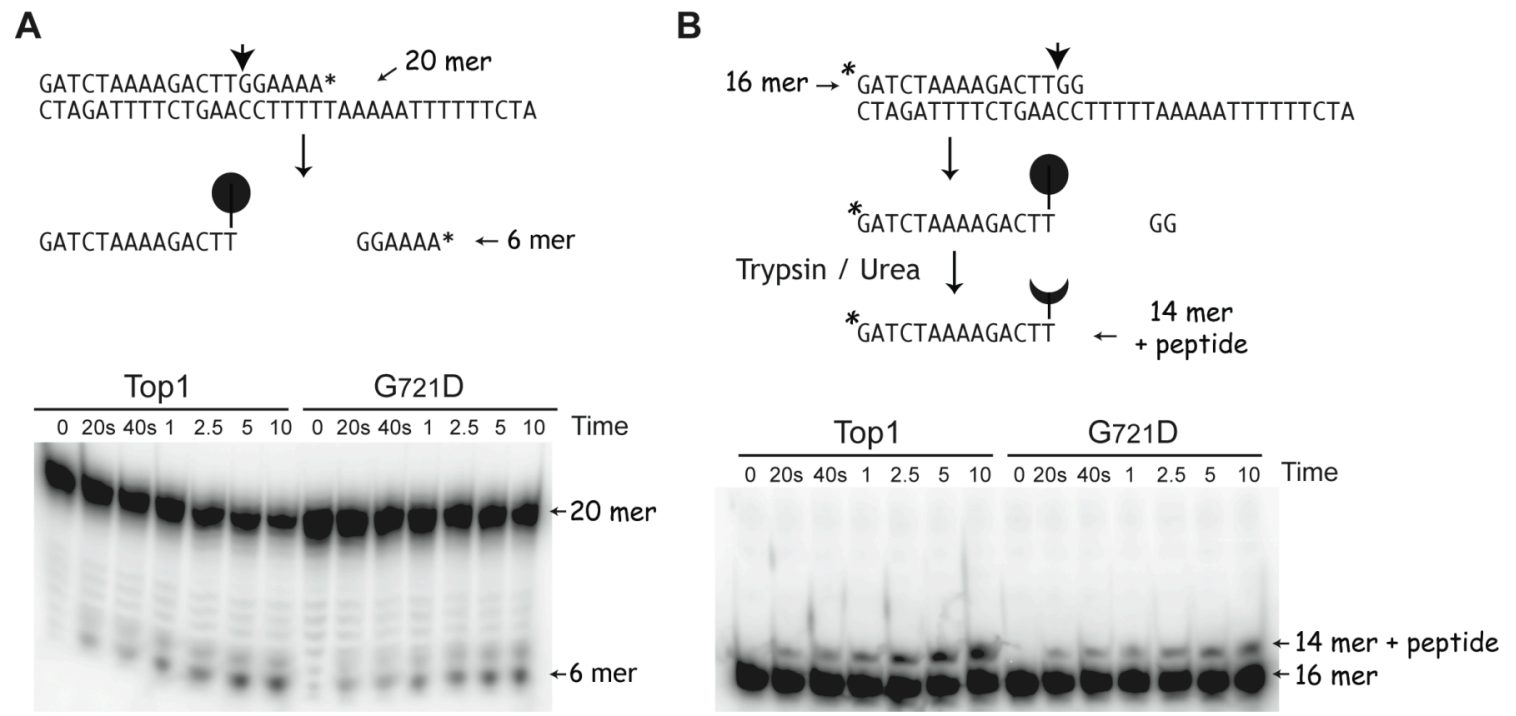

FIG. 3.6. Truncated suicide substrates reduce the rate of DNA cleavage by

Top $1 \mathbf{G}^{721} \mathbf{D}$. $A$, as diagrammed, Top 1 cleavage of a suicide substrate containing a 20 mer 3 'end labeled scissile strand (indicated by $\downarrow$ ), liberates a ${ }^{32} \mathrm{P}$-labeled 6 mer. In this assay, equal concentrations of Top 1 and $T o p 1 \mathrm{G}^{721} \mathrm{D}$ were incubated the labeled 20 mer at room temperature. At the times indicated, reaction aliquots were terminated with $0.5 \%$ SDS at $75{ }^{\circ} \mathrm{C}$, the cleavage products were resolved in a $20 \%$ polyacrylamide/7M Urea gel and visualized by PhosphorImaging. $B$, as diagrammed, Top1 cleavage (indicated by $\downarrow$ ) of a suicide substrate containing a 5'-radiolabeled oligonucleotide ( 16 mer) liberates a GGdinucleotide and traps the covalent $\left[\gamma-{ }^{32} \mathrm{P}\right] \mathrm{DNA}-\mathrm{Top} 1 \mathrm{p}$ complex (intact Top1 is indicated by ). Trypsin digestion of the covalent complex in the presence of $2 \mathrm{M}$ Urea generates a 7 amino acid peptide covalently linked to a 14-mer. In this assay, equal concentrations of Top1 and Top $1 \mathrm{G}^{721} \mathrm{D}$ were incubated with the suicide substrate and at the times indicated, reaction aliquots were terminated with $0.5 \% \operatorname{SDS}$ at $75{ }^{\circ} \mathrm{C}$, ethanol precipitated, and the trypsinized products resolved in a denaturing gel and visualized by PhosphorImaging. 
complexes $(103,156,176)$. Thus, the rate of DNA cleavage by Top1 can be approximated by the kinetics of 6-mer accumulation. With this scissile DNA strand, the observed rates of DNA cleavage were comparable for wild-type Top1 and the $\mathrm{G}^{721} \mathrm{D}$, $\mathrm{G}^{721} \mathrm{E}$ and $\mathrm{G}^{721} \mathrm{~N}$ mutants (Fig. 3.6.A and data not shown). When monitoring DNA religation by Top1, a shorter scissile DNA strand is typically used (see schema in Fig. 3.7.A) $(103,156,176)$. Here, the dissociation of a dinucleotide facilitates the subsequent annealing of a complementary oligonucleotide, which provides the $5^{\prime} \mathrm{OH}$ necessary to resolve the trapped covalent complex. In this assay, the suicide oligonucleotide is 5'end labeled so both the extent of DNA cleavage and religation can be monitored. However, to use this assay, we first needed to determine if the removal of four nucleotides 3 ' to the site of DNA scission altered the rate of enzyme catalyzed DNA cleavage. Using the strategy depicted in Fig. 3.6.B, Top1-DNA covalent complex formation is monitored by the accumulation of a peptide-linked 14-mer, produced by trypsin digestion of the reaction products in the presence of urea (177). Contrary to results obtained with the longer DNA substrate, the truncated suicide substrate revealed a decreased rate of DNA cleavage by the $\mathrm{G}^{721} \mathrm{D}$ mutant, relative to that obtained with wild-type Top1 (Fig. 3.6.B). These data suggest that the rate of DNA cleavage by the $\mathrm{G}^{721} \mathrm{D}$ mutant is affected by the extent of duplex DNA structure 3' to site of DNA cleavage.

We next considered the effects of Gly ${ }^{721}$ mutations on the rate of Top1-catalyzed DNA religation, using the strategy depicted in Fig. 3.7.A. Following the trapping of covalent Top1-DNA complexes with the 16-mer suicide substrate, a complementary religation oligonucleotide is added (time $=0$ ). At the times indicated, the reactions were 
A

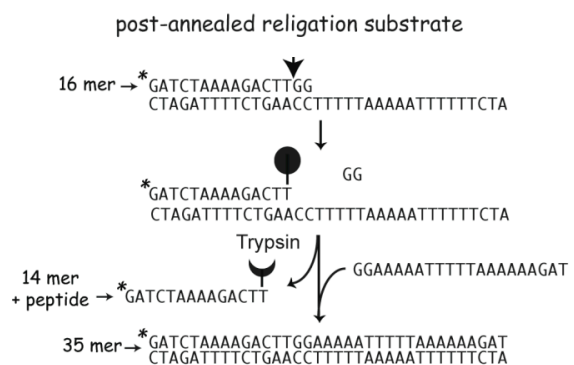

B

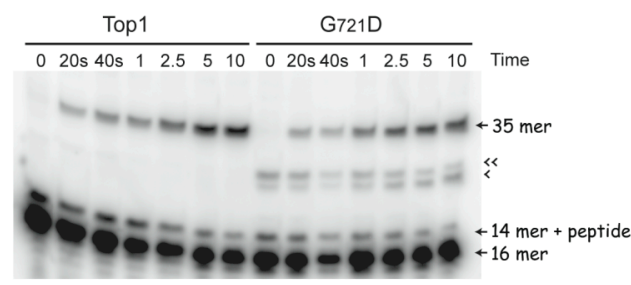

C

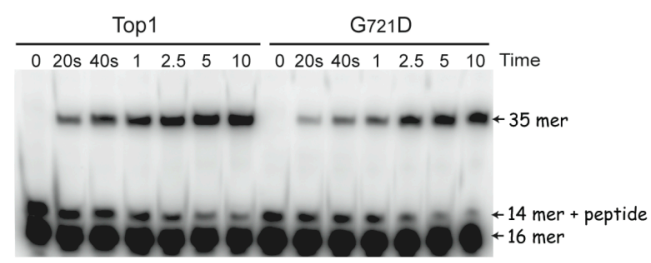

D

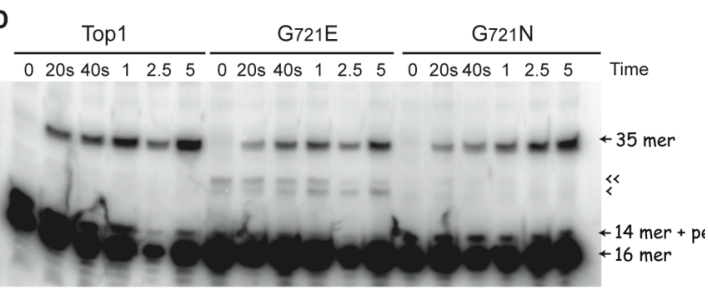

FIG. 3.7. Distinct patterns of Top1-DNA tryptic digests obtained in religation reactions suggest $\mathbf{G}^{721} \mathbf{D}$-induced alterations in active site structure. $A$, in the postannealed religation assay, the same suicide substrate diagrammed in Fig. 4.6.B, is used to generate covalent $\left[\gamma_{-}{ }^{32} \mathrm{P}\right] \mathrm{DNA}-\mathrm{Top} 1$ complexes. At $\mathrm{t}=0$, a 21 mer religation oligonucleotide (complementary to the 5 'end of the nonscissile strand) is added. As shown in $B$ and $C$, the resolution of the covalent Top1-DNA complex to generate a ${ }^{32} \mathrm{P}$ labeled 35-mer can be followed by the conversion of the tryptic 14 mer + peptide to the 35 mer. $B, C$, equal concentrations of Top 1 , Top $1 \mathrm{G}^{721} \mathrm{D}$, Top $1 \mathrm{G}^{721} \mathrm{E}$ and $T o p 1 \mathrm{G}^{721} \mathrm{~N}$ were incubated with the suicide substrate for 15 minutes at room temperature. At $t=0$, the 21 mer religation oligonucleotide was added. At the times indicated, reaction aliquots were terminated with SDS at $75^{\circ} \mathrm{C}$, treated with trypsin (in the absence of urea), resolved in $16 \%$ polyacrylamide/7 M Urea gels visualized using a PhosphoImager. $D$, as for the reactions shown in $B$, equal concentrations of Top 1 and $T o p 1 G^{721} \mathrm{D}$ were incubated in the post-annealed religation assay. However, the reaction products were digested with trypsin in the presence of $2 \mathrm{M}$ Urea prior to 16\% polyacrylamide/7 M Urea gel electrophoresis. 
terminated with SDS at $75^{\circ} \mathrm{C}$, and the relative levels of uncleaved 16-mer, religated 35mer and covalent Top1-DNA intermediate were assessed in denaturing gels following trypsin digestion. These reactions were initially carried out in the absence of urea, sincetrypsin digestion of wild-type Top1 (Fig. 3.7.B) efficiently liberated a 7 amino peptide (INY ${ }^{727}$ IDPR) covalently linked to the radiolabeled 14 -mer, which migrates slower than the 16-mer suicide oligonucleotide. However, a distinct pattern of bands was obtained in tryptic digests of covalent complexes formed by Top $1 \mathrm{G}^{721} \mathrm{D}$. As seen in Fig. 3.7.B, two additional bands are observed at 0 time, with the slower migrating band $($ labeled $<<$ ) being more prominent. Following addition of the religation oligonucleotide, the accumulation of the faster migrating band (labeled $<$ ) paralleled that of the religated 35-mer. Similar results were obtained in assays performed at $150 \mathrm{mM} \mathrm{KCl}$ (data not shown). These extra bands do not result from illegitimate religation events. Rather, they represent incomplete tryptic digests of the covalent $T o p 1 \mathrm{G}^{721} \mathrm{D}$-DNA complexes, as trypsin digestion of these complexes in the presence of urea produced a single 7 residue peptide linked to the radiolabeled 14-mer (Fig. 3.7.C). Moreover, digestion with proteinase $\mathrm{K}$, which cleaves at sites distinct from trypsin, failed to yield multiple bands (data not shown). Nevertheless, if one corrects for differences in the level of covalent complexes formed at $\mathrm{t}=0$, then there was no difference in the rate of DNA religation (accumulation of 35-mer following the addition of the religation oligonucleotide) catalyzed by Top1 versus Top $1 \mathrm{G}^{721} \mathrm{D}$ (Fig. 3.7.C).

To determine if altered tryptic digests coincided with increased enzyme sensitivity to CPT, similar analyses of Top $1 \mathrm{G}^{721} \mathrm{E}$ and $\mathrm{Top} 1 \mathrm{G}^{721} \mathrm{~N}$ were performed. All three mutants produced lower levels of covalent enzyme-DNA intermediates at $t=0$, with no 
obvious defect in DNA religation (Fig. 3.7.B and C). However, only in the case of the more CPT sensitive Top $1 \mathrm{G}^{721} \mathrm{E}$ mutant enzyme were the longer peptide-linked oligonucleotides obtained; these bands were not evident with the less CPT sensitive Top $1 \mathrm{G}^{721} \mathrm{~N}$ (Fig. 3.7.D). Thus, substituting acidic residues for $\mathrm{Gly}^{721}$ appears to enhance CPT poisoning of Top 1 and alter the geometry of the active site in the covalent complex. We next asked if the presence of extended duplex DNA 3' to the site of DNA cleavage, which affects the rate of DNA cleavage by Top $1 \mathrm{G}^{721} \mathrm{D}$ (Fig. 3.6), also impacts enzyme active site architecture and/or rates of DNA religation. To address these questions, the religation oligonucleotide was annealed to the suicide DNA substrate prior to the addition of Top1, as diagrammed for the "pre-annealed religation substrate in Fig. 3.8.A. Although this substrate contains a short DNA flap, the same suicide oligonucleotide used in the "post-annealed religation substrate" (Fig. 3.7.A) was used to restrict the overlap to 2 nucleotides. In addition to 14 mer + peptide, trypsin digestion of the reaction products produced a longer Top $1 \mathrm{G}^{721} \mathrm{D}$-derived peptide covalently attached to the cleaved DNA (< in Fig. 3.8.B), corresponding to the lower band obtained with the post-annealed religation substrate in Fig. 3.7. Thus, the presence of DNA annealed to the non-scissile strand induced distinct alterations in mutant enzyme active site architecture when probed by trypsin digestion. Moreover, the accumulation of religated DNA products was also diminished in $T o p 1 \mathrm{G}^{721} \mathrm{D}$ reactions containing the pre-annealed substrates, relative to that observed with wild-type Top1 (Fig. 3.8.B) or Top $1 \mathrm{G}^{721} \mathrm{D}$ incubated with post-annealed substrates. However, because the pre-annealed assay does not uncouple cleavage from religation, it is difficult to determine if the presence of the dinucleotide flap and/or duplex DNA also affects the rate of DNA cleavage. 
A

pre-annealed religation substrate

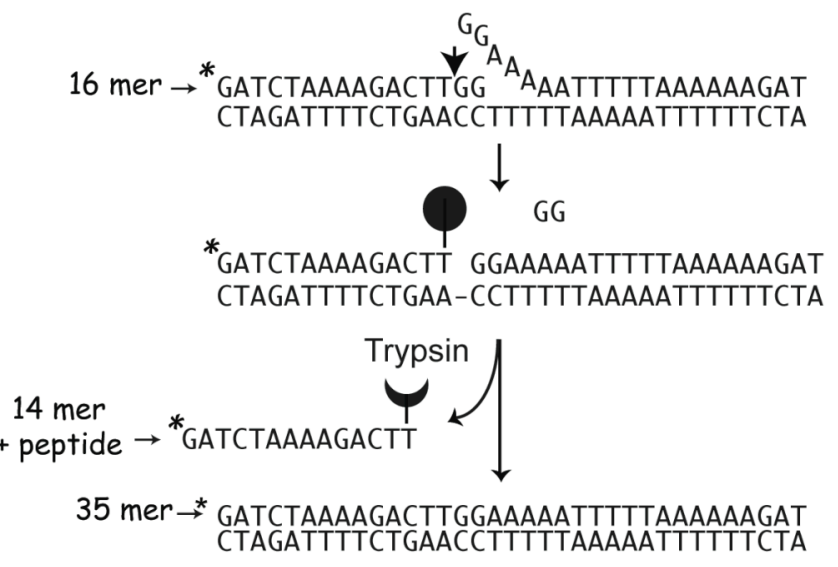

B

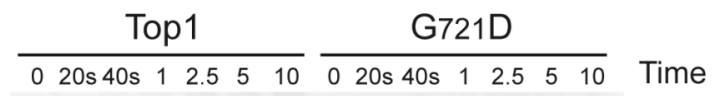

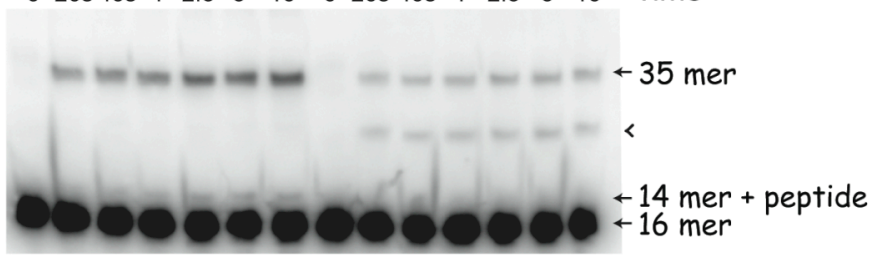

C

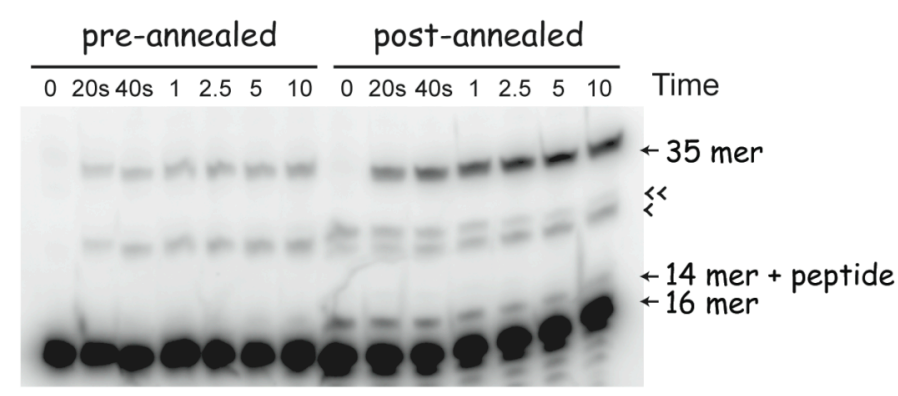

FIG. 3.8. Duplex DNA, 3 ' to the site of DNA scission, impacts Top $1 G^{721} D$ enzyme architecture and the rate of enzyme-catalyzed DNA religation. $A$, in the pre-annealed religation assay, the 21 mer religation oligonucleotide diagrammed in Fig.7A is annealed prior to the addition of enzyme. DNA cleavage by Top1 liberates a GG dinucleotide to allow formation of the 35 mer. $B$, equal concentration of Top 1 and $T o p 1 G^{721} \mathrm{D}$ were added to pre-annealed religation assays at $\mathrm{t}=0$. At the times indicated, reaction aliquots were quenched with SDS at $75^{\circ} \mathrm{C}$. Covalently linked Top1 proteins were hydrolyzed with trypsin (in the absence of Urea) and the reaction products were resolved in $16 \%$ polyacrylamide/7M Urea gels. $C$, tryptic digests of $\mathrm{Top} 1 \mathrm{G}^{721} \mathrm{D}$, incubated in pre-annealed or post-annealed religation assays, were resolved by $16 \%$ polyacrylamide/7M Urea gel electrophoresis. 
Nevertheless, the alterations in tryptic digests of the covalent enzyme-DNA intermediates induced by the various DNA substrates coincided with increased mutant enzyme sensitivity to CPT.

Based on these findings, we hypothesize that the increased CPT sensitivity of the $\mathrm{Gly}^{721}$ mutant enzymes results from conformational changes within the Top1 active site that limits linker domain flexibility and enhances CPT binding to the covalent enzyme complex. In particular, mutation of the highly flexible Gly might extend the $\alpha$-helical structure of residues N-terminal to the active site Tyr, as seen in the structures shown in Fig.3.1. One prediction of this model is that, independent of side chain charge, an increased tendency for $\alpha$-helical structure at position 721 would augment CPT poisoning of Top1. Estimates of the intrinsic helix-forming tendencies of various amino acid residues indicate that while Gly has a low propensity to form $\alpha$-helices, Ala has the highest (178). Indeed, substituting Ala for Gly ${ }^{721}$ (in Top $1 \mathrm{G}^{721} \mathrm{~A}$ ) induced a 5-fold increase in cell sensitivity to CPT, relative to cells expressing wild-type Top1, which was only slightly less than the 10 -fold increase induced by the $\mathrm{G}^{721} \mathrm{D}$ mutation (Fig. 3.9).

\subsection{DISCUSSION}

The architecture of Top1 includes a flexible linker domain, comprised of an extended coiled coil that positions the C-terminal active site Tyr domain within the catalytic pocket formed in concert with the central protein clamp. Biochemical, X-ray crystallographic and molecular dynamic simulation data suggest that the integrity and 


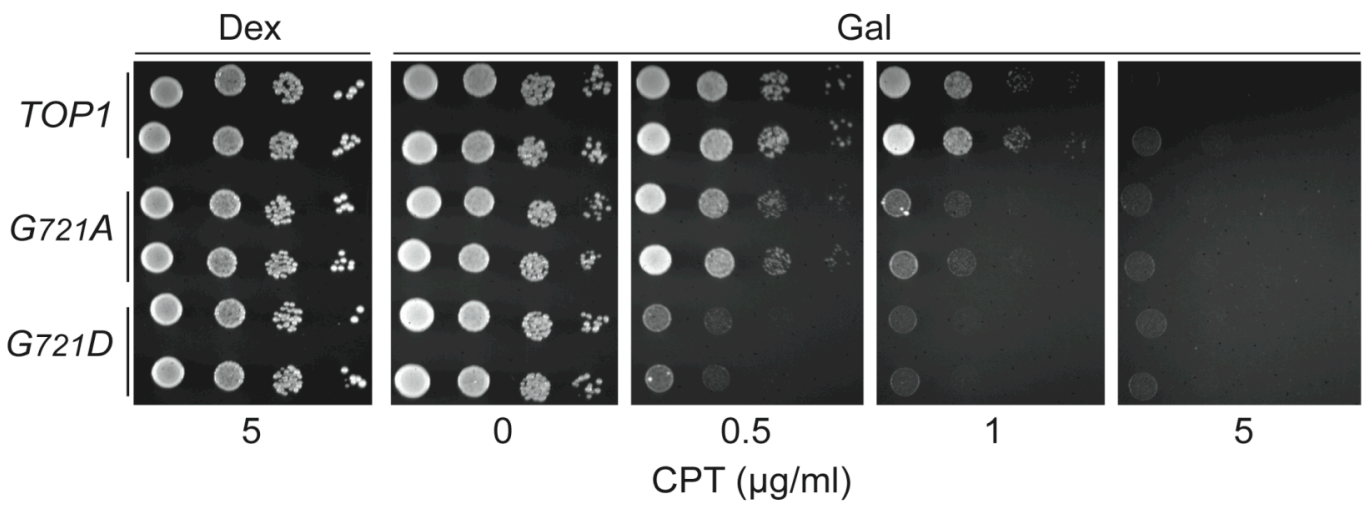

FIG. 3.9. A propensity for a-helical structure at residue 721 enhances Top1 sensitivity to CPT. Exponential cultures of top $1 \Delta$ cells, transformed with YCpGAL1TOP1, YCpGAL1-top $1 \mathrm{G}^{721} \mathrm{~A}$ or YCpGAL1- $\mathrm{G}^{721} \mathrm{D}$, were serially 10 -fold diluted and spotted onto SC-uracil plates containing $25 \mathrm{mM}$ Hepes (pH 7.2), 0.1\% DMSO, dextrose (Dex) or galactose (Gal), and the indicated concentrations of CPT. Cell viability assessed following incubation at $30^{\circ} \mathrm{C}$. 
flexibility of the linker domain within the Top1 clamp are critical determinants of enzyme sensitivity to the CPT class of chemotherapeutics $(59,61,62,173,175)$. Increasing the flexibility of the human Top1 linker domain, either by mutation $\left(\mathrm{A}^{653} \mathrm{P}\right)$ (62) or by physically uncoupling the linker domain from the Top1 protein clamp (in reconstituted Topo70 preparations) (61), reduces enzyme sensitivity to CPT. In co-crystal structures of human Topo70 and DNA, the presence of CPT analogues appears to restrict the movement of the linker domain thereby enabling the structural determination of this coiled-coil. This contrasts with the mobility of the linker domain within the covalent Topo70-DNA complex in the absence of drug. Close inspection of active site architecture within these structures revealed an extension of a short $\alpha$-helical structure, spanning Ser $^{719}$ through the active site Tyr (human $\operatorname{Tyr}^{723}$ ) in the absence of drug, to include Leu $^{716}, \mathrm{Gly}^{717}$ and $\mathrm{Thr}^{718}$ in the presence of drug (see Fig. 3.1). These data suggest a dynamic interplay between active site $\alpha$-helical structure and linker flexibility attends drug binding to the covalent Topo70-DNA complex. Such long range molecular interactions between the Top1 linker and active site in mediating enzyme sensitivity to CPT are also supported by recent studies where the cytotoxicity induced by a defect in human Top1p-catalyzed DNA religation (due to the active site mutation $\mathrm{T}^{722} \mathrm{~A}$ ) was suppressed by the increased linker flexibility of the $\mathrm{A}^{653} \mathrm{P}$ mutation (174). Based on these considerations, we hypothesized that the presence of this conserved Gly residue within the active site of the enzyme (Gly ${ }^{717}$ in human Top1; Gly ${ }^{721}$ in yeast Top1) functions as a flexible hinge to facilitate the alterations in active site geometry and linker domain flexibility that impact CPT poisoning of Top1. Indeed, our analyses of $\mathrm{Gly}^{721}$ mutations in yeast Top1, in vitro and in vivo, support this model and suggest that the charge and 
geometry of amino acid side chains at this position directly impact the helical structure of the region preceding the active site Tyr within the covalent enzyme-DNA complex and concommitantly the intrinsic sensitivity of Top1 to CPT.

First, our findings indicate that the introduction of a bulky aromatic residue (Phe) or $\beta$ branched aliphatic side chain (Val) decrease the specific activity and CPT sensitivity of Top1. By contrast, the introduction of an acidic side chain, with carboxyl groups at the $\beta$ or $\gamma$ carbon in $\mathrm{G}^{721} \mathrm{D}$ and $\mathrm{G}^{721} \mathrm{E}$, respectively, dramatically enhanced Top1 sensitivity to CPT, without any obvious alterations in specific activity in plasmid DNA relaxation assays. In this case, side chain charge was important, as the increased CPT sensitivity of cells expressing Top1 mutants engineered with the amide forms of these residues $\left(G^{721} \mathrm{~N}\right.$ and $\left.\mathrm{G}^{721} \mathrm{Q}\right)$ or a $\gamma$ branched aliphatic side chain $\left(\mathrm{G}^{721} \mathrm{~L}\right)$ was only evident in the absence of the DNA damage checkpoint. However, the tendency of residues at this position to form $\alpha$-helices also appears to be a critical determinant of Top1 sensitivity to CPT, as mutating Gly ${ }^{721}$ to Ala [estimated to have the highest propensity for helix formation (178)] also increased cell sensitivity to CPT. Together, our data support the following rank order of mutant-induced increased in Top1p sensitivity to CPT: $\left(G^{721} E, G^{721} D\right)>$ $\mathrm{G}^{721} \mathrm{~A}>\left(\mathrm{G}^{721} \mathrm{~N}, \mathrm{G}^{721} \mathrm{Q}, \mathrm{G}^{721} \mathrm{~L}\right)>$ wild-type $>\mathrm{G}^{721} \mathrm{~V}>\mathrm{G}^{721} \mathrm{~F}$.

Second, our studies also suggest mutation-induced alterations in enzyme active site architecture coincide with increased $C P T$ sensitivity. The $\mathrm{G}^{721} \mathrm{D}, \mathrm{G}^{721} \mathrm{E}$ mutants exhibited the greatest CPT sensitivity in vivo and in vitro, as well as specific alterations in tryptic digests of covalent enzyme-DNA complexes formed in DNA religation assays. A comparison of potential trypsin cleavage sites in yeast and human Top1 with the pattern of DNA-bound tryptic fragments obtained with yeast and human Top1 mutants (Fig. 3.7, 
3.8 and data not shown) allows for an accurate determination of the alterations in trypsin digestion. In the residues spanning the active site tyrosine of yeast Top1, $\underline{\underline{K}}^{712}$ EENSQYSLG ${ }^{721} \mathrm{TS}^{724} \underline{I N Y}^{727} \mathrm{IDP} \underline{\underline{R}}$, trypsin digestion of SDS-denatured wild-type Top1-DNA covalent complexes typically yields a 7 residue peptide (INY ${ }^{727}$ IDPR) covalently attached to a 5' end labeled 14 mer oligonucleotide via a 3'phosphotyrosyl linkage. Mutation of $\mathrm{Gly}^{721}$ to Asp or Glu $\left(\mathrm{G}^{721} \mathrm{D}\right.$ or $\mathrm{G}^{721} \mathrm{E}$, respectively) limits trypsin digestion at $\mathrm{K}^{724}$ to yield longer peptides linked to the DNA. One possible explanation is that the $\mathrm{G}^{721} \mathrm{D}$ and $\mathrm{G}^{721} \mathrm{E}$ mutations alter the $\alpha$-helical structure of the active site so as to restrict trypsin digestion of the partially denatured proteins. The presence of an acidic side chain may also preclude efficient trypsin digestion at $\mathrm{K}^{724}$. Nevertheless, the changes in enzyme active site geometry suggested by the altered tryptic digests coincided with increased Top1 sensitivity to CPT- alterations in trypsin digestion were only observed with the more CPT sensitive $\mathrm{G}^{721} \mathrm{D}$ or $\mathrm{G}^{721} \mathrm{E}$ mutants and not with the less CPT sensitive $\mathrm{G}^{721} \mathrm{~N}$ mutant.

The presence of duplex DNA 3' to the site of DNA scission also altered the rates of Top $1 \mathrm{G}^{721} \mathrm{D}$ catalyzed DNA cleavage and religation, as well as the pattern of tryptic digests, suggesting that downstream protein-DNA contacts impact dynamic interactions between the active site and the linker. Indeed, the linker of human Top 1 is 10 -fold more resistant to limited proteolysis when the enzyme is noncovalently bound to duplex DNA, consistent with linker-DNA contacts 3' to the site of DNA scission (28). While mutationinduced alterations in Top1 structure await X-ray structure determination, our studies implicate the conserved $\mathrm{Gly}^{721}$ residue as a flexible hinge within the active site of Top1 
that enable linker domain flexibility and the structural alterations that accompany drug binding of the covalent enzyme-DNA complex. 


\section{CHAPTER 4: DISTINCT STRUCTURAL DOMAINS REGULATE DNA TOPOISOMERASE I ACTIVITY AND SENSITIVITY TO CAMPTOTHECIN}

\subsection{INTRODUCTION}

DNA topoisomerases catalyze changes in DNA topology by a concerted mechanism of DNA strand cleavage and religation $(3,7,179)$. Eukaryotic DNA topoisomerase I (Top1) relaxes positive and negative supercoils generated during processes such as replication, transcription, recombination and chromosome condensation. The enzyme transiently cleaves a single strand of duplex DNA to form a covalent DNA-(3'-phosphotyrosyl)-enzyme intermediate, which allows rotation of the non-covalently held DNA end around the non-scissile strand to relieve torsional and/or flexural strain in the DNA. The 5'-OH end of the cleaved DNA strand acts as a nucleophile in a second transesterification reaction to resolve the covalent Top1-DNA intermediate and religate the DNA phosphodiester backbone.

Top1 is the sole cellular target of the camptothecin (CPT) class of chemotherapeutics $(6,55,57)$. Several CPT analogs, including topotecan (TPT) and the pro-drug CPT-11, have demonstrated remarkable antitumor activity against adult and pediatric tumors, with additional analogs in clinical development $(73,170)$. CPTs target the enzyme by reversibly stabilizing a covalent Top1-DNA intermediate. In co-crystal structures of human Topo70 (a C-terminal fragment of human Top1 that lacks the Nterminus) covalently linked to DNA, intercalation of CPT (or TPT) into the protein- 
linked DNA nick is stabilized by base-stacking interactions between the planar drug with -1 (upstream) and +1 (downstream) DNA bases (59). Drug binding also induces an $\sim 3.6 \AA$ shift of the downstream base pairs, which displaces the reactive 5'-OH of the cleaved strand and prevents DNA religation. Recent studies further demonstrate that drug binding to the covalent enzyme-DNA complex preferentially slows Top1 uncoiling of positively supercoiled DNA (180). In S-phase, the ternary CPT-Top1-DNA complex itself or the positive supercoils that accumulate in advance of the replication machinery as a consequence of CPT poisoning of Top1 poise an obstacle to replication fork progression and trigger the formation of the irreversible DNA lesions that induce cell death (180, 181).

Human cells encode two closely related Top1 proteins, where distinct N-terminal sequences direct the nuclear or mitochondrial localization of the monomeric enzyme (182). In yeast and human cells, the nuclear Top1 is highly conserved in terms of reaction mechanism and sensitivity to CPT $(3,152,179)$. Indeed, sequence similarities, limited proteolytic digestion, enzyme reconstitution experiments with Top1 fragments and crystallographic data describe a unique enzyme architecture, comprising four distinct domains $(12,15,27,28,42,59)$. A divergent $\mathrm{N}$-terminal domain is dispensable for in vitro activity (31), but is a determinant of intracellular localization (127) and Top1 interactions with other proteins and DNA $(32,33,36)$. The conserved core domain constitutes a clamp that circumscribes duplex DNA and contains all of the catalytic residues except the active site Tyr, which is located in the conserved C-terminal domain. In co-crystal structures of human Topo70 with duplex DNA, a salt bridge between opposing protein loops completes the tight packing of the DNA within the Top1 core. 
However, some flexibility or breathing of the protein clamp is necessary for enzyme catalysis since crosslinking of the active-site proximal loops ("Lips"), which locks the Top1 clamp closed, inhibits DNA rotation within the covalent complex (41). Top1 also contains a linker domain consisting of a pair of extended $\alpha$-helices that connect the Cterminal domain with protein core to appropriately position the active site Tyr within the catalytic pocket of the enzyme. The linker is dispensable for DNA relaxation activity in vitro as an active enzyme can be reconstituted with individually purified core and Cterminal domain polypeptides (39).

The N-terminal and linker domains vary considerably in size and amino acid sequence. Yet, despite this apparent lack of conservation, nuclear Top1 enzymes typically contain an $\mathrm{N}$-terminus rich in charged residues $(3,7)$. Crystallographic studies reveal the human Top1 linker forms as an extended coiled coil with positively charged residues on the surface facing the bound duplex DNA $(27,59)$. The longer linker domain of yeast Top1 (yTop1) is also predicted to form $\alpha$-helices that would extend twice the distance from the protein clamp (110 $\AA$ ), with a similar distribution of positive charges. In Topo70 structures, there are few contacts between the linker and the core clamp/C-terminus of the enzyme, giving the linker domain a high degree of flexibility (60). The unique architectural features of nuclear Top1 enzymes - the conserved protein clamp with a flexible linker positioning the active site tyrosine domain within the active site of the protein core - and the paucity of structural information on the poorly conserved Nterminus, raise a number of questions concerning the effect of linker flexibility on enzyme activity and/or CPT sensitivity and whether the N-terminus impacts these aspects of Top1 function. 
Several lines of evidence suggest the integrity and/or flexibility of the linker domain affects enzyme sensitivity to CPT. For instance, in X-ray structures of human Topo70-DNA covalent complexes, the linker domain is apparent only when the drug is bound and extends out from the active site at an oblique angle to the DNA (59). A similar architecture is seen when Topo70 is noncovalently bound to DNA (27). However, in covalent Topo70-DNA complexes formed in the absence of drug, these flexible coiled coil structures were not resolved (59). When Topo70 was reconstituted from separate polypeptides comprising the core domain and the linker/C-terminal domain, the reconstituted enzyme was resistant to CPT (61). In this case, the absence of a physical connection between the linker and core domains induced a shift in the cleavage-religation equilibrium towards religation. Mutation of linker domain residue $\mathrm{Ala}^{653}$ to Pro (in Top $1 \mathrm{~A}^{653} \mathrm{P}$ ) induced a similar increase in DNA religation to render Top1 resistant to CPT (62). Molecular dynamic simulations suggested these alterations in enzyme catalysis were due to increased linker flexibility. Moreover, the ability of the $\mathrm{A}^{653} \mathrm{P}$ mutation to suppress the lethal phenotype induced by the self-poisoning $\mathrm{T}^{722} \mathrm{~A}$ mutation provides direct evidence for the physical interaction between the Top1 linker and active site of the enzyme (183).

To address how linker orientation impacts active site geometry to alter enzyme catalysis, we began by mutating conserved residues in yeast Top1 (hereafter referred to as yTop1) that lie at the junction between the linker and C-terminal domains. We previously reported that substitution of $\mathrm{Gly}^{721}$ with residues having a higher propensity to form $\alpha$ helices, such as Asp, increase yTop1 sensitivity to CPT (184). Our findings supported a model in which this conserved Gly residue provides a flexible hinge within the active site 
to promote alterations in linker structure that accompany CPT binding of the covalent Top1-DNA complex. Here we report that substitutions of the corresponding Gly ${ }^{717}$ residue in human Top1 (hTop1) also increased enzyme sensitivity to CPT in vitro, with similar alterations in active site architecture. Surprisingly, however, these mutations failed to induce CPT hypersensitivity in yeast cells. This apparent disconnect between intrinsic enzyme sensitivity to CPT and drug-induced cytotoxicity in vivo, led us to investigate the contributions of the divergent $\mathrm{N}$-terminal and linker domains to enzyme activity and CPT sensitivity in vivo. Using a series of chimeric Top1 enzymes, involving reciprocal swaps of the yeast and human N-termini and linkers, we determined that intrinsic enzyme sensitivity to CPT is dictated by the composition of the conserved core and C-terminal domains. However, it is the functional interaction of the N-terminus and linker domains that regulate enzyme activity in vivo. Taken together, our findings provide important new insights into how alterations in enzyme architecture impact the catalytic activity and drug sensitivity of Top1 in cells.

\subsection{EXPERIMENTAL PROCEDURES}

\subsubsection{Chemicals, yeast strains and plasmids}

Camptothecin was purchased from Sigma, and stock solutions of $4 \mathrm{mg} / \mathrm{ml}$ in DMSO were stored at $-20{ }^{\circ} \mathrm{C} .\left[\alpha-{ }^{32} \mathrm{P}\right]$ Cordycepin 5 '-triphosphate was obtained from PerkinElmer Life Sciences (Boston, MA). Anti FLAG $\mathrm{M}_{2}$ affinity gel, FLAG peptide and $\mathrm{M}_{2}$ monoclonal antibodies were purchased from Sigma-Aldrich. 


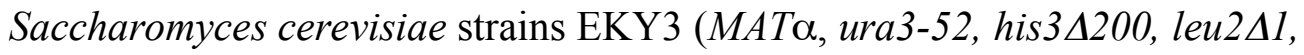
$\operatorname{trp} 1 \Delta 63$, top $1 \Delta:: T R P 1)$ and MMY3 (EKY3, rad9A::hisG) have been described (113). In plasmids YCpGAL1-yTOP1 and YCpGAL1-hTOP1, wild-type yeast TOP1 and human $T O P 1$ cDNA were expressed from the galactose-inducible GAL1 promoter in an $A R S / C E N, U R A 3$ vector $(41,113)$, while hTop1 was constitutively expressed from the $S$. cerevisiae TOP1 promoter in YCpSc-hTOP1 (180). All of the constructs used in these studies contained an N-terminal Flag epitope (DYKDDD) recognized by the M2 monoclonal antibody. The "h" and "y" designations distinguish human and yeast TOPl, respectively. To generate the corresponding YCpSc-yTOP1 vector, yTOP1 sequences were excised with BamHI and $\mathrm{XbaI}$ and ligated under the yeast TOP1 promoter in the pRS416-based vector YCpSc $\bullet U$ (185). To generate htop $1 G^{717} D$, htop $1 G^{717} Q$, htop $1 L^{716} E$ and $\mathrm{h}$ top $1 L^{716} Q$ mutants, where residue $\mathrm{Gly}^{717}$ was mutated to Asp and Gln, and Leu ${ }^{716}$ to Glu and Gln, respectively, a SacII-ClaI hTOPI DNA, ligated into pRS416, was mutagenized using a Quickchange mutagenesis kit (Stratagene, La Jolla, CA) and pairs of complementary oligonucleotides based on the following sequences: GD (5'AAACAGATTGCCCTGGACACCTCCAAACTCAAT-3'); GQ (5'AAACAGATTGCCCTGCAAACCTCCAAACTCAAT-3'); LE (5'AATAAACAGATTGCCGAGGGAACCTCCAAACTC-3') and LQ (5'AATAAACAGATTGCCCAGGGAACCTCCAAACTC-3'). Mutant sequences were excised with SacII and ClaI and ligated into YCpGAL1-hTOP1 to yield YCpGAL1htop $1 \mathrm{G}^{717} \mathrm{D}$, YCpGAL1-htop $1 \mathrm{G}^{717} \mathrm{Q}$, YCpGAL1-htop1L ${ }^{716} \mathrm{E}$ and YCpGAL1htop $1 \mathrm{~L}^{716} \mathrm{Q}$. Yeast-human chimeras were generated using homologous recombination of PCR-generated chimeric junctions as previously described (185) (see Table 4.1 for 
TABLE. 4.1. Composition of yeast/human Top1 chimeras ${ }^{\mathrm{a}}$.

\begin{tabular}{|c|c|c|}
\hline Chimera $^{b}$ & Composition $^{\mathrm{c}}$ & Junction sequence $^{\mathrm{d}}$ \\
\hline$(\mathrm{hN})$-yTop1 & $\begin{array}{l}\text { hTop1(1-191)- } \\
\text { yTop1(120-769) }\end{array}$ & DKKVPEPDNK/EDKKAKEE \\
\hline$\left(\mathrm{hN} N^{209}\right)$-yTop1 & $\begin{array}{l}\text { hTop1(1-209)- } \\
\text { yTop1(138-769) }\end{array}$ & EQKWKWWEEE/NEDDTIKW \\
\hline (yN)-hTop1 & $\begin{array}{l}\text { yTop1(1-119)- } \\
\text { hTop1(192-765) }\end{array}$ & EKKKREEEEEE/KKKPKKEEEQKW \\
\hline$\left(\mathrm{yN}^{120}\right)$-hTop1 & $\begin{array}{l}\text { yTop1(1-120)- } \\
\text { hTop1(201-765) }\end{array}$ & EKKKREEEEEEE/QKWKWWEEERY \\
\hline (hL,hC)-yTop1 & $\begin{array}{l}\text { yTop1(1-560)- } \\
\text { hTop1(635-765) }\end{array}$ & VAILCNHQR/APPKTFEKSMM \\
\hline (hL)-yTop1 & $\begin{array}{l}\text { yTop1(1-560)- } \\
\text { hTop1(635-713)- } \\
\text { yTop1(717-769) }\end{array}$ & QATDREENK/QVSLGTSKI \\
\hline (yL,yC)-hTop1 & $\begin{array}{l}\text { hTop1(1-634)- } \\
\text { yTop1(561-769) }\end{array}$ & VAILCNHQR/TVTKGHAQTVEK \\
\hline (yL)-hTop1 & $\begin{array}{l}\text { hTop1(1-634)- } \\
\text { yTop1(561-716)- } \\
\text { hTop1(713-765) }\end{array}$ & QLKDKEENS/QVSLGTSKI \\
\hline
\end{tabular}

${ }^{a}$ Chimeric Top1 enzymes were generated by homologous recombination of PCR products. Chimera junctions were engineered in the PCR primers. Primer sequences are available upon request.

${ }^{\mathrm{b}}$ The heterologous Top1 protein domain (in parenthesis) is shaded blue for yeast residues and pink for human residues, core and the corresponding C-terminal sequences are in black and are designated $\mathrm{h}$ for human or $\mathrm{y}$ for yeast. $\mathrm{N}, \mathrm{L}$ and $\mathrm{C}$ refer to $\mathrm{N}$-terminal, linker and $\mathrm{C}$-terminal domains, respectively. For example, $(\mathrm{hN})$-yTop1 contains the human $\mathrm{N}$-terminus and the yeast core, linker and C-terminal domains. (yL,yC)-hTop1 contains the human $\mathrm{N}$-terminus and core domains, with the yeast linker and $\mathrm{C}$-terminus.

${ }^{\mathrm{c}}$ The numbers indicate the yeast or human amino acid residues contained in the chimeras, with 1 refering to the first Met residue. The pink and blue shading is as described above. The (hL,hC)-yTop1 chimera was used to generate (hL)-yTop1, while the (yL,yC)-hTop1 chimera was used to generate (yL)-hTop1.

${ }^{\mathrm{d}}$ Residues spanning the chimera junctions (indicated by a /) are indicated using the color coding described above. 
primer design and junctions). To generate the Gly ${ }^{721}$ to Asp substitution in the yeast top 1

chimera containing the human linker $\left[(h L)-y t o p 1 G^{72 I} D\right]$, a pair of complementary primers was used based on the following sequence: 5'-

AAACAGGTTTCACTGGACACTTCCAAAATCAAT. In all cases, mutant sequences were confirmed by DNA sequencing. Yeast cells were transformed and maintained using standard methods.

\subsubsection{Yeast cell sensitivity to CPT}

To assess cell sensitivity to CPT, exponential cultures of EKY3 (top1 1 ) and MMY3 (top14, rad94) yeast cells transformed with the indicated YCpGAL1-TOP1 or YCpSc-TOP1 vector, were adjusted to an $A_{595}=0.3$, serially 10 -fold diluted and aliquots were spotted onto SC-uracil agar plates containing $25 \mathrm{mM}$ Hepes (pH 7.2), $2 \%$ dextrose or galactose and the indicated concentration of CPT in a final $0.125 \%$ DMSO. Colony formation was assessed following incubation at $30^{\circ} \mathrm{C}$.

\subsubsection{DNA topoisomerase I expression, purification and activity assays}

Wild-type, mutant and chimeric enzymes were purified from galatose-induced cultures of top $1 \Delta$ yeast cells expressing plasmid encoded Top 1 proteins as described $(156,184)$. Briefly, partially purified proteins were obtained by successive ammonium sulfate fractionations followed by phosphocellulose chromatography, as described (95). Top1 protein fractions were adjusted to a final $30 \%$ glycerol and stored at $-20{ }^{\circ} \mathrm{C}$. Human Top1 and $\mathrm{hTop} 1 \mathrm{G}^{717} \mathrm{D}$ were further purified to homogeneity by applying the partially purified fractions to an anti flag M2 affinity gel (Sigma Chemical Co., St. Louis 
MO) and eluting the Top1 proteins with excess Flag peptide in TBS (50 mM Tris, $\mathrm{pH}$ 7.4, $150 \mathrm{mM} \mathrm{KCl})$. Peptide was removed by adding the purified fractions to a phosphocellulose column and eluting the protein in TEEG buffer (50 mM Tris, pH7.4, 1 mM EDTA, 1mM EGTA, 10\% glycerol, $1 \mathrm{M} \mathrm{KCl)}$ and protease inhibitors (180). These fractions were diluted with $50 \%$ glycerol and stored at $-20{ }^{\circ} \mathrm{C}$. Protein integrity was assayed in immunoblots as described $(103,184)$.

Top1 activity was assessed in plasmid DNA relaxation reaction as described (99, 103). Briefly, serial 10-fold dilutions of purified Top1 proteins, corrected for concentration, were incubated in $20 \mu \mathrm{l}$ reaction volumes with $0.3 \mu \mathrm{g}$ of negatively supercoiled plasmid pHC624 DNA in $20 \mathrm{mM}$ Tris (pH 7.5), $10 \mathrm{mM} \mathrm{MgCl} 2,0.1 \mathrm{mM}$ EDTA, $50 \mu \mathrm{g} / \mathrm{ml}$ gelatin and the indicated concentration of $\mathrm{KCl}$ at $30{ }^{\circ} \mathrm{C}$ for 30 minutes. The reactions products were resolved in a $1.2 \%$ agarose gels and visualized following staining with ethidium bromide.

\subsubsection{DNA and suicide cleavage assays}

Intrinsic enzyme sensitivity to CPT was assessed in DNA cleavage assays as previously described (156). Briefly, a DNA fragment containing a high affinity Top1

cleavage site was uniquely $3{ }^{32} \mathrm{P}$-end-labeled, incubated with equal concentrations of Top1 proteins in cleavage buffer [20 mM Tris ( $\mathrm{pH} 7.5), 10 \mathrm{mM} \mathrm{MgCl} 2,0.1 \mathrm{mM}$ EDTA, $50 \mathrm{mM} \mathrm{KCl}$, and $50 \mu \mathrm{g} / \mathrm{ml}$ gelatin] and increasing concentrations of CPT in a final $4 \%$ (v/v) DMSO for $10 \mathrm{~min}$ at $30{ }^{\circ} \mathrm{C}$. The reactions were terminated with SDS at $75^{\circ} \mathrm{C}$, digested with proteinase $\mathrm{K}$ and resolved in $8 \%$ polyacrylamide/7 $\mathrm{M}$ urea gels. The cleavage products were visualized using a PhosphorImager. 
Oligonucleotide-based assays were used to assess relative rates of DNA cleavage catalyzed by wild-type and mutant proteins. As previously described, several approaches were used $(103,184)$. First, substrates contained either a truncated scissile strand 5'GATCTAAAAGACTT $\downarrow$ GG-3' (where $\downarrow$ denotes the Top1 cleavage site) or a longer scissile strand containing a bridging phosphorothiolate linkage at the site of cleavage (inducated by “s” in 5' GATCTAAAAGACTTsGGAAAATTTTTAAAAAAGATC-3'). The phosphorothiolates were synthesizes as described (186). These suicide oligonucleotides were 5 ' - end-labeled with $\left[\gamma^{-}{ }^{32} \mathrm{P}\right] \mathrm{ATP}$ and T4 polynucleotide kinase, while the complementary oligonucleotide 5'GATCTTTTTTAAAAATTTTTCCGTCTTTTAGATC-3' was phosphorylated with cold ATP. Equimolar concentrations of the oligonucleotides were annealed in $10 \mathrm{mM}$ Tris $(\mathrm{pH}$ 7.8), $100 \mathrm{mM} \mathrm{NaCl}, 1 \mathrm{mM}$ EDTA by heating to $90{ }^{\circ} \mathrm{C}$ followed by slow cooling to room temperature. The suicide substrate ( $3 \mathrm{pmol} /$ reaction) was incubated with equal concentrations of Top1 proteins in Cleavage buffer at room temperature and, at the times indicated, aliquots were treated with $0.5 \% \mathrm{SDS}$ at $75{ }^{\circ} \mathrm{C}$ to terminate the reactions. Following an ethanol precipitation, the covalently linked protein was digested with trypsin, in the presence or absence of $2 \mathrm{M}$ Urea, and the remaining substrate and peptidelinked oligonucleotides were resolved in $16 \%$ polyacrylamide/7M Urea gels and visualized by PhosphoImage analysis. A third substrate was used to monitor the release of a 6 mer downstream of the Top1 cleavage site (99). For these assays, a slightly longer scissile strand 5'-GATCTAAAAGACTT $\downarrow$ GGAAA-3', 3'-end-labeled with $\left[{ }^{32} \mathrm{P}\right]$ cordycepin using terminal deoxynucleotide transferase, was annealed to equimolar amounts of the same complementary strand as described above. Equal concentrations of 
Top1 proteins were incubated with the suicide substrate (20 fmoles/reaction) in Cleavage buffer at room temperature. Aliquots were quenched with SDS at $75^{\circ} \mathrm{C}$ at the times indicated and the reaction products were resolved in $20 \%$ polyacrylamide/7M Urea gels and visualized with a Phosphoimager.

\subsubsection{DNA religation assays}

To assess the relative rates of Top1-catalyzed DNA religation, the 5'-end labeled DNA suicide substrate containing 5'-GATCTAAAAGACTT $\downarrow$ GG-3' was used in two oligonucleotide-based religation assays as described (184). In the post-annealed religation assay, the suicide substrate was incubated for 15 minutes at room temperature with equal concentrations of Top1 proteins in Cleavage buffer to generate covalent Top1-DNA complexes. Religation was initiated at time $=0$ by the addition of a 10 -fold excess of a religation oligonucleotide (5'-GGAAAAATTTTTAAAAAAGATC-3'), which is complementary to the non-scissile strand downstream of the site of DNA scission. In the pre-annealed religation assay, the same suicide substrate was incubated with a 10 -fold excess of religation oligonucleotide for 15 min prior to the addition of Top1 proteins. In both assays, aliquots were taken at various times, processed as described above for the 5'end labeled suicide cleavage reactions. Where indicated, the reaction products were hydrolyzed with trypsin in the absence of urea prior to probe for changes in enzyme active site architecture. 


\subsection{RESULTS}

\subsubsection{Mutation of $\mathrm{Gly}^{717}$ in human Top1 does not alter CPT-induced toxicity in yeast cells, yet enhances enzyme sensitivity to CPT in vitro}

Crystallographic and biochemical data suggest that the physical connection of the Top1 linker to the C-terminal domain / protein clamp as well as the flexibility of the linker domain are critical determinants of enzyme sensitivity to CPT $(59,61,62,184)$. In the co-crystal structure of the covalent human Topo70-DNA complex, Gly ${ }^{717}$ lies at one end of a short $\alpha$-helix that extends to the active site $\mathrm{Tyr}^{723}$, which is covalently linked to the DNA (59). In the ternary Topo70-DNA-TPT structure, this $\alpha$-helix extends to include $\mathrm{Gly}^{717}$, as well as the preceding Leu ${ }^{716}$. This seemingly subtle structural transition in active site architecture coincides with a more dramatic restriction in linker flexibility. The coiled coil linker, which was not resolved in the absence of drug, is now oriented at an oblique angle to the DNA in the presence of TPT. A similar orientation of the linker is also seen when Topo70 is noncovalently bound to DNA (Fig. 4.1) and in covalent Topo70-DNA complexes bound with other CPT analogs $(27,85,175)$.

We recently reported that mutating the corresponding yeast $\mathrm{Gly}^{721}$ residue to amino acids with a higher propensity to form $\alpha$-helices, for example Ala, Asp or Glu, enhanced yTop1 CPT sensitivity, while the $\beta$-carbon branched Val, or the aromatic Phe at this position induced CPT resistance (184). With the Asp and Glu substituents, CPT hypersensitivity corresponded to detectable alterations in active site architecture, as probed by limited tryptic digests. Based on such considerations, we hypothesized that this 

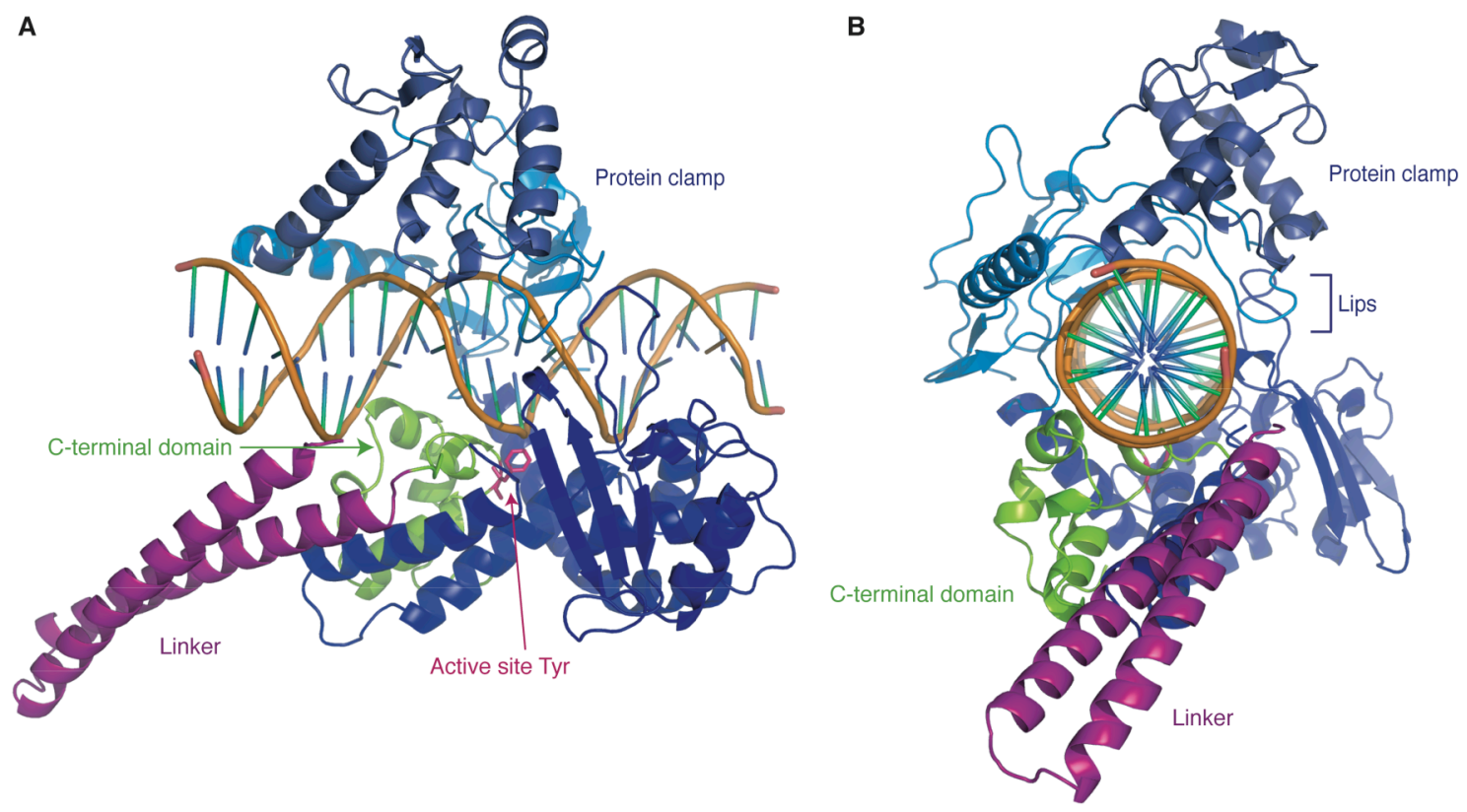

FIG. 4.1. The structure of a C-terminal $70 \mathrm{kD}$ fragment of human DNA topoisomerase I (Topo70) in a noncovalent complex with a 22 base pair DNA duplex. $A$. Ribbon diagram of PDB file 1E36, viewed perpendicular to helical axis of the DNA duplex (backbone is in orange), the core domain of Topo70 forms a protein clamp (shades of blue). The linker domain (purple) extends from the core at an oblique angle to the DNA and the C-terminal domain is in green. The active site Tyr is mutated to Phe (indicated in magenta). $B$. The same structure rotated $90^{\circ}$ around the vertical axis and viewed down the DNA helix. Figures were made with PyMol. 
residue constitutes a flexible hinge, where changes in active site architecture at this junction impact the orientation and flexibility of the linker domain (184). Our initial focus on yeast Top1 avoided potential complications of heterologous human Top1 expression in yeast top $1 \Delta$ strains; nevertheless, the underlying assumption was that the high degree of sequence conservation within the enzyme active site was predictive of structure. Yet, as all structural determinations of Top1 active site architecture are limited to human Topo $70(27,59,85,175)$, we asked if the same amino acid substitutions in the human enzyme elicited similar dynamic transitions in active geometry and alterations in CPT sensitivity.

We first engineered mutations of the conserved $\mathrm{Leu}^{716}$ in hTop1, analogous to those known to render yTop1 resistant to CPT (184). As shown in Fig. 4.2.A, mutation of $\mathrm{Leu}^{716}$ to $\mathrm{Gln}\left(\right.$ in $\mathrm{L}^{716} \mathrm{Q}$ ) or Glu (in $\mathrm{L}^{716} \mathrm{E}$ ) induced a CPT resistant phenotype when these mutant htop 1 alleles were expressed from the galactose-inducible GAL1 promoter in yeast top $1 \Delta$ cells. Cells expressing either hTop 1 mutant enzyme were resistant to 10 -fold higher CPT concentrations than cells expressing comparable levels of wild-type hTop1. In plasmid DNA relaxation and DNA cleavage assays performed with purified proteins, the specific activity of the $\mathrm{L}^{716} \mathrm{E}$ mutant was reduced $\sim 10$-fold relative to that of wildtype hTop1, which corresponded to a proportional decrease in enzyme sensitivity to CPT in vitro (shown in Fig. 4.2.B and C). Similar results were obtained with the corresponding mutation in yTop1, $\mathrm{L}^{720} \mathrm{E}$, albeit with more severe effects on enzyme activity and CPT sensitivity in vitro (184). This apparent conservation of hTop1 $\mathrm{Leu}^{716}$ mutant function, in vivo and in vitro, contrasted with the phenotypic consequences of mutating the conserved $\mathrm{Gly}^{717}$ in $\mathrm{hTop} 1$ to Asp $\left(\mathrm{G}^{717} \mathrm{D}\right)$ or $\mathrm{Gln}\left(\mathrm{G}^{717} \mathrm{Q}\right)$. Expression of the corresponding yeast 

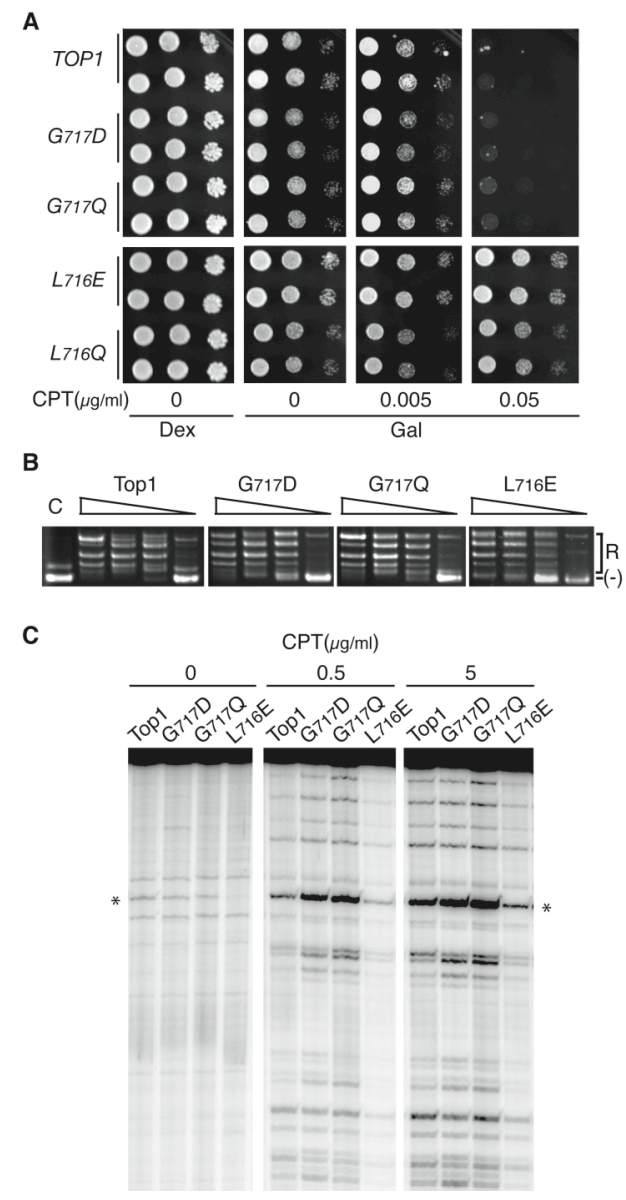

FIG. 4.2. Mutation of $\operatorname{Gly}^{717}$ to $\operatorname{Asp}\left(G^{717} D\right)$ or Gln $\left(G^{717} Q\right)$ enhanced hTop1 sensitivity to CPT in vitro, but not in yeast. $A$. Exponentially growing cultures of top $1 \Delta$ cells, transformed with the indicated YCpGAL1-htop1 constructs, were adjusted to an $A_{595}=0.3$, serially 10 -fold diluted, and aliquots were spotted onto SC -uracil agar plated supplemented with $25 \mathrm{mM}$ HEPES (pH 7.2), 0.1\% DMSO and either dextrose (Dex), galactose $(\mathrm{Gal})$ or galactose plus the indicated concentration of CPT. Cell viability was assessed following incubation at $30^{\circ} \mathrm{C}$. B. Equal concentrations of purified hTop1 proteins were serially 10 -fold diluted and incubated with $0.3 \mu \mathrm{g}$ of negatively supercoiled pHC624 DNA in a plasmid DNA relaxation assay as detailed in Materials and Methods. The reaction products were resolved by agarose gel electrophoresis and visualized after ethidium bromide staining. The relative positions of relaxed (R) and supercoiled (-) DNA topoisomers are indicated. $\mathrm{C}$ is plasmid DNA control. $C$. Equal concentrations of hTop1 and mutant proteins were incubated with a single $3{ }^{32} \mathrm{P}$-end labeled DNA fragment in a DNA cleavage reaction containing a final $4 \%(\mathrm{v} / \mathrm{v})$ DMSO and indicated concentration of CPT. After a $10 \mathrm{~min}$ incubation at $30^{\circ} \mathrm{C}$, the covalent complexes were trapped with SDS at $75{ }^{\circ} \mathrm{C}$ and treated with proteinase $\mathrm{K}$. The reaction products were resolved in an $8 \%$ polyacrylamide/7M urea gel and visualized using a PhosphoImager. The asterisk (*) indicates a high affinity Top1 cleavage site. 
mutants, $\mathrm{G}^{721} \mathrm{D}$ in a top $1 \Delta$ strain or $\mathrm{G}^{721} \mathrm{Q}$ in an isogenic strain deleted for the Rad9 DNA damage checkpoint (top 1 $\Delta, \operatorname{rad} 9 \Delta)$, enhanced cell sensitivity to CPT by a factor of 10 . However, galactose-induced yeast top $1 \Delta$ cells expressing wild-type hTop $1, \mathrm{hTop} 1 \mathrm{G}^{717} \mathrm{D}$, or $\mathrm{hTop} 1 \mathrm{G}^{717} \mathrm{Q}$ exhibited similar sensitivity to $\mathrm{CPT}$, over a range of drug concentrations (Fig. 4.2.A). Similar results were also obtained in other genetic backgrounds, such as top $1 \Delta$,rad9 $\Delta$ cells (data not shown). The specific activity of the purified human $\mathrm{G}^{717} \mathrm{D}$ and $\mathrm{G}^{717} \mathrm{Q}$ mutant enzymes was similar to that of wild-type hTop1 (Fig. 4.2.B), yet exhibited an $\sim 10$-fold increase in CPT-induced covalent Top1-DNA complex formation (Fig. 4.2.C). Surprisingly, the increased CPT sensitivity of the human $\mathrm{G}^{717} \mathrm{D}$ and $\mathrm{G}^{717} \mathrm{Q}$ mutant enzymes evident in vitro mirrored that of the corresponding yeast mutants, yet failed to alter yeast cellular responses to CPT. This apparent disconnect between mutant enzyme activity in vitro and in vivo prompted us to consider whether the mechanism of enhanced mutant enzyme sensitivity to CPT was conserved from yeast to human or if the different phenotypes resulted from distinct functional interactions of the yeast and human enzymes in vivo.

\subsubsection{Mutation-induced changes in the DNA cleavage-religation equilibrium and structural alteration of $\mathrm{hTop} 1 \mathrm{G}^{717} \mathrm{D}$}

To assess the mechanism of enhanced CPT poisoning of human $T o p 1 \mathrm{G}^{717} \mathrm{D}$ in vitro, a series of oligonucleotide-based DNA substrates were used to uncouple enzyme catalyzed DNA cleavage from DNA religation. Biochemical and structural studies indicate that CPT reversibly stabilizes the covalent Top1-DNA intermediate by reducing the rate of DNA religation $(3,59,170)$. Indeed, recent single molecule studies suggest 
that dissociation of the drug occurs prior to the resolution of the Top1-DNA complex (180). Thus, an increase in CPT sensitivity could result from increased covalent complex formation, due to mutation-induced alterations in DNA cleavage or religation, or an increase in the affinity of the drug for the mutant Top1-DNA intermediate. For example, mutation of either the conserved Asn immediately N-terminal to the active site Tyr in yTop $1 \mathrm{~N}^{726} \mathrm{H}$ or $\mathrm{Thr}^{722}$ in yTop $1 \mathrm{~T}^{722} \mathrm{~A}$, increased the steady state levels of the covalent intermediates such that these "self-poisoning" enzymes were toxic to yeast and hypersensitive to CPT in vitro $(99,103,113)$. Using suicide substrates to uncouple Top1catalyzed DNA cleavage from religation, we established that yTop $1 \mathrm{~N}^{726} \mathrm{H}$ exhibited an increased rate of DNA cleavage without a corresponding increase in DNA religation, while the cytotoxicity of the $\mathrm{T}^{722} \mathrm{~A}$ mutant derived from a decrease in DNA religation, much like that induced by CPT (103). Similar alterations in enzyme catalysis were observed with the corresponding hTop1 mutants (103). However, in the DNA cleavage assays shown Fig. 4.2.C, there were no obvious alterations in steady state levels of $\mathrm{G}^{717} \mathrm{D}$ or $\mathrm{G}^{717} \mathrm{Q}$ covalent complexes relative to that observed with wild-type hTop1, in the absence of CPT.

To determine the basis for the increase in intrinsic CPT sensitivity of $\mathrm{hTop} 1 \mathrm{G}^{717} \mathrm{D}$, we first addressed the relative rates of DNA cleavage. As diagrammed in Fig. 4.3, two truncated oligonucleotide-based suicide substrates were used. While both contained a Top1 high affinity cleavage site, they differed in the number of bases present downstream from the site of scission. In our studies of $y T o p 1 \mathrm{G}^{721} \mathrm{D}$, the relative rates of mutant enzyme catalyzed DNA cleavage increased with the length of the scissile DNA strand (184). However, as shown in Fig. 4.3.A and B, the human $\mathrm{G}^{717} \mathrm{D}$ mutant enzyme 

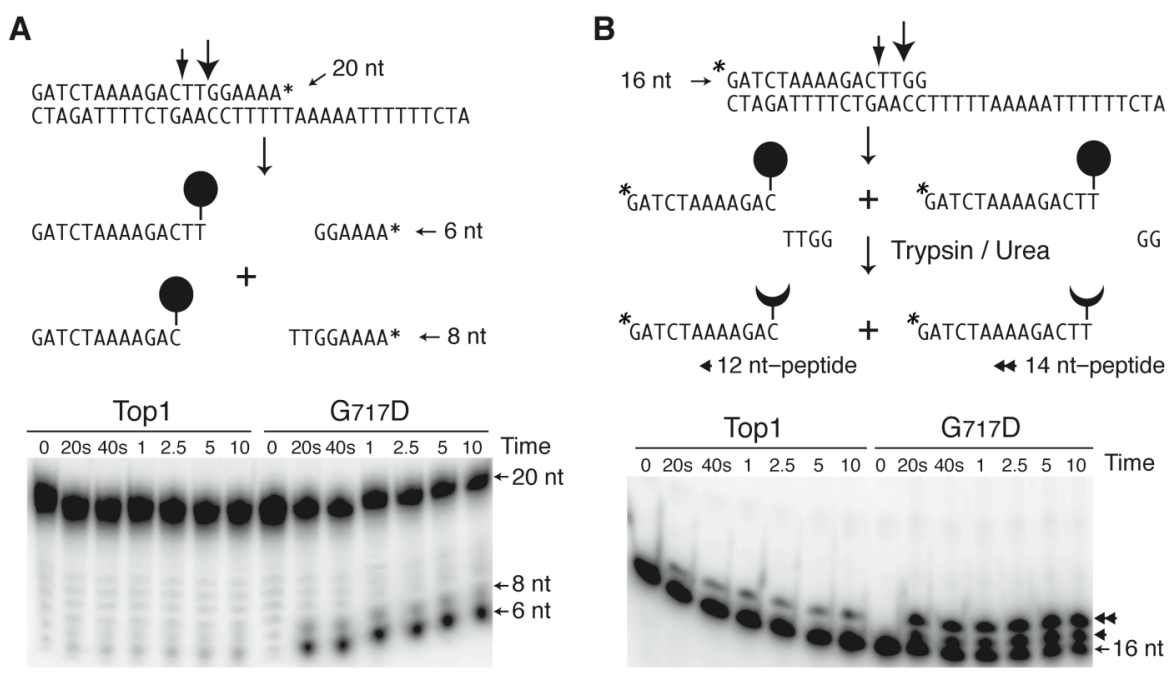

FIG. 4.3. $h T 0 p 1 G^{717} D$ exhibits increased rates of DNA cleavage. $A$. As diagrammed, Top1 cleavage of a 3'-labeled 20 nucleotide ( $20 \mathrm{nt}$ ) oligomer containing the high affinity cleavage site for Top1 (indicated by $\downarrow$ ), liberates a ${ }^{32} \mathrm{P}$-labeled 6 nucleotide oligomer ( 6 $\mathrm{nt})$. The smaller arrow indicates a second cleavage site. In this assay, equal concentrations of hTop 1 and $h T o p 1 \mathrm{G}^{721} \mathrm{D}$ were incubated with the labeled $20 \mathrm{nt}$ and, at the times indicated, aliquots were treated with SDS at $75^{\circ} \mathrm{C}$ and the cleavage products were resolved in a $20 \%$ polyacrylamide/7M Urea gel and visualized with a PhosphorImager. B. A 5'-radiolabeled oligonucleotide (16 nt) containing the high affinity cleavage site (indicated by $\downarrow$ ) was incubated with hTop1 or hTop $1 G^{721} \mathrm{D}$. A second cleavage site is indicated by the smaller arrow. Top1-catayzed cleavage traps the covalent $\left[\gamma^{32} \mathrm{P}\right] \mathrm{DNA}$-Top 1 complex (intact Top1 is indicated by $)$. Trypsin digestion of the covalent complex in the presence of $2 \mathrm{M}$ Urea generates a 7 amino acid peptide (O) covalently linked to a $14 \mathrm{nt}$ (or $12 \mathrm{nt}$ with complexes formed at the second cleavage site). At the times indicated, reaction aliquots were terminated with SDS at $75^{\circ} \mathrm{C}$, ethanol precipitated and trypsinized. Reaction products were resolved and visualized as above 
exhibited dramatically elevated rates of DNA cleavage independent of DNA strand length. Enhanced DNA cleavage was assessed by the accumulation of the free $6 \mathrm{nt}$ fragment in Fig. 4.3.A and the generation of a 14 nt-peptide complex following trypsin digestion of the reaction products in the presence of urea in Fig. 4.3.B. In both cases, the mutant enzyme also catalyzed the cleavage of DNA at a second site, 2 nucleotides upstream from the high affinity site (Fig. 4.3.A and B). Similar results were also obtained using full length duplex DNA that contains a 5' bridging phosphorothiolate at the site of DNA scission (see Fig. 4.4). Enzyme cleavage of this suicide substrate yields a 5' SH, which is an inefficient in the subsequent transesterification necessary to religate the DNA (186). In all of these assays, increased rates of DNA cleavage were observed with the human $\mathrm{G}^{717} \mathrm{D}$ mutant, relative to wild-type hTop1 (Fig. 4.3.A, 4.3.B and Fig. 4.4). DNA cleavage and the dissociation of Top1 from duplex DNA are rate-limiting steps in Top1 catalysis (168). Alterations in Top1 protein binding of DNA shift the optimal salt concentration for plasmid DNA relaxation (99). However, as the specific activity of the $\mathrm{G}^{717} \mathrm{D}$ mutant mirrored that of wild-type hTop 1 over a wide range of salt concentrations, a defect in $\mathrm{hTop} 1 \mathrm{G}^{717} \mathrm{D}$ binding of DNA is unlikely (data not shown). On the other hand, since the rate of mutant enzyme-catalyzed DNA scission was enhanced, then a comparable increase in DNA religation rate would be necessary to maintain similar steady state levels of covalent Top1-DNA complexes. To address this question, enzymecatalyzed DNA religation was assessed in a post-annealed religation assay, as diagrammed in Fig. 4.5.A. In this assay, the formation of covalent enzyme-DNA complexes (using the suicide substrate in Fig. 4.3.B) is followed by the addition of a complementary religation oligonucleotide, such that Top1-catalyzed DNA religation can 

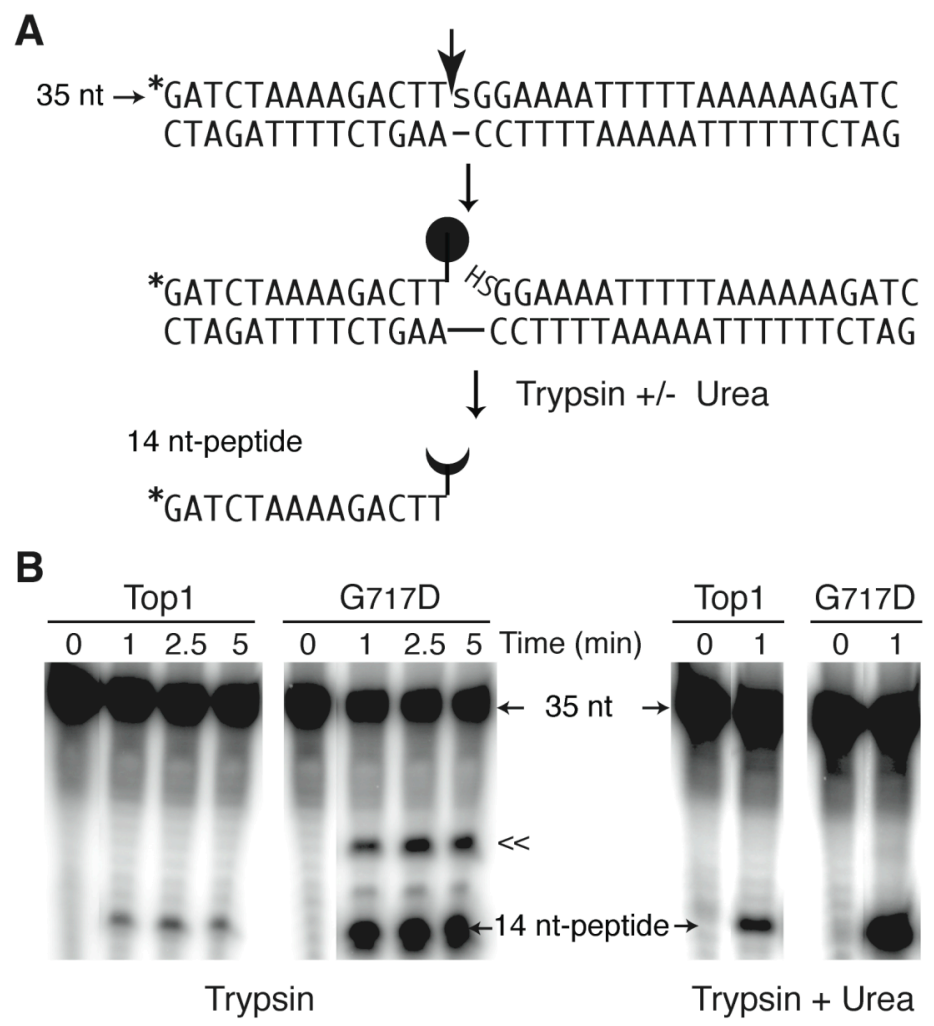

FIG. 4.4. Substitution of $\mathrm{Gly}^{717}$ with Asp increases the rate of Top1-catalyzed DNA cleavage. $A$. Schematic representation of a DNA cleavage assay using a 5' end labeled suicide substrate $(35 \mathrm{nt})$ that contains a 5 ' bridging phosphorothioate (designated s) at the high affinity Top 1 cleavage site (indicated by $\downarrow$ ). The 5'-SH generated by Top1 cleavage at this site prevents DNA religation, such that the accumulation of covalent Top1-DNA complexes approximates the rate of DNA scission. $B$. Equal concentrations of hTop1 or hTop $1 \mathrm{G}^{717} \mathrm{D}$ were incubated with $5^{\prime}$ end labeled suicide substrate diagrammed in $A$. At the times indicated aliquots were treated with SDS at $75^{\circ} \mathrm{C}$, ethanol precipitated and digested with trypsin in the absence or presence of urea. Cleavage products were resolved in a $16 \%$ polyacrylamide/ $7 \mathrm{M}$ urea gels and visualized with a PhospoImager. The relative mobility of the starting substrate (35 nt), the cleaved DNA strand linked to a 7 amino acid peptide following trypsin digestion (14 nt-peptide) and a longer peptide linked to the 14 nt $(<<)$ resulting from of a partial tryptic digest. 
A post-annealed religation substrate
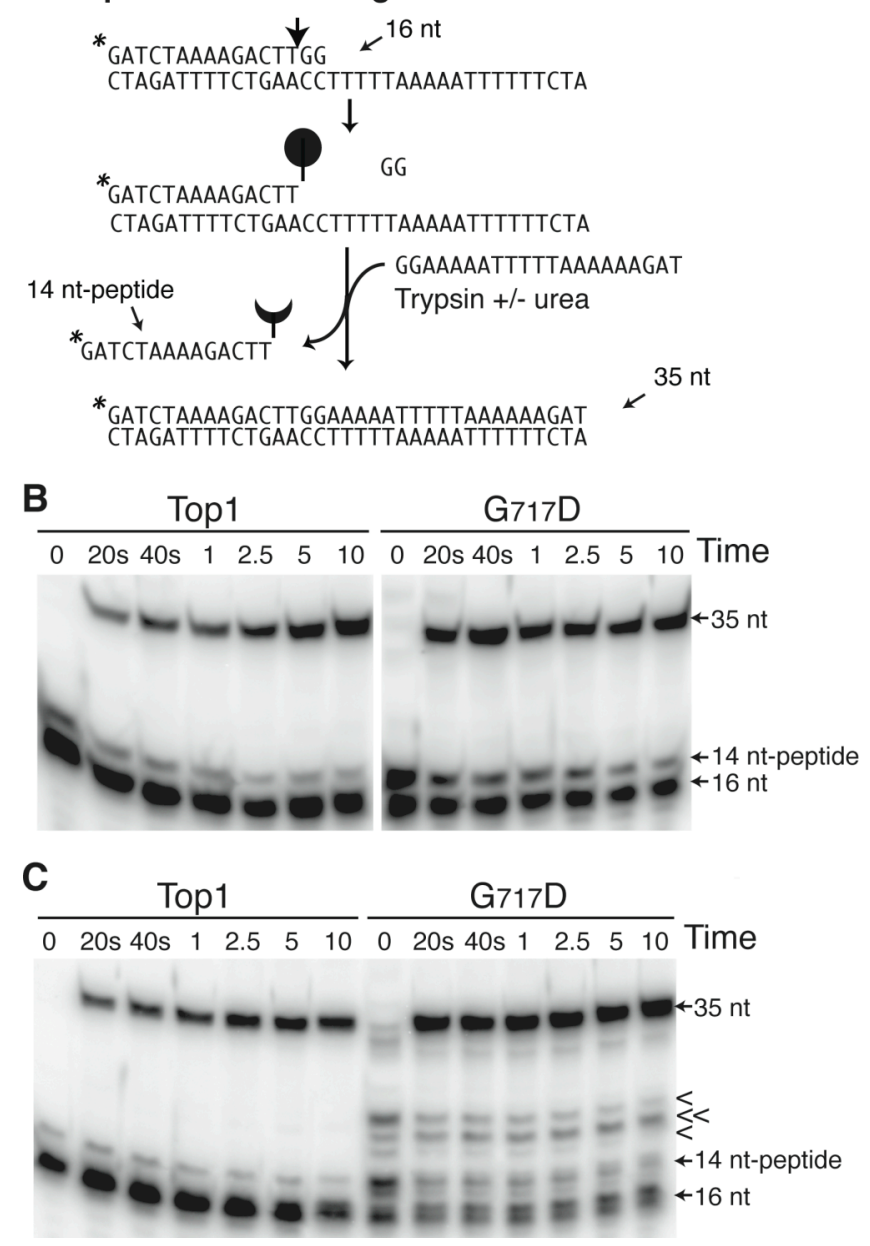

FIG. 4.5. Tryptic digests of $h \operatorname{Top} \mathrm{G}^{717} \mathrm{D}$-DNA complexes during DNA religation suggest mutant-induced alterations in active site structure. $A$. In a post-annealed religation assay, the same suicide substrate diagrammed in Fig. 4.2.B was used to generate covalent $\left[\gamma_{-}{ }^{32} \mathrm{P}\right] \mathrm{DNA}$-Top 1 complexes. At 0 time, a 21 nt oligonucleotide (complementary to the 5 'end of the nonscissile strand) is added to initiate religation. As shown in $B$ and $C$, the resolution of covalent hTop1-DNA complexes to generate a ${ }^{32} \mathrm{P}-$ labeled $35 \mathrm{nt}$ product can be followed by the conversion of the tryptic 14 nt-peptide to a 35 nt. $B$. Equal concentrations of purified hTop 1 and $h T o p 1 G^{717} \mathrm{D}$ were incubated with the suicide substrate for 15 minutes at room temperature. The $21 \mathrm{nt}$ was added at $\mathrm{t}=0$ and reaction aliquots were terminated at the indicated times with SDS at $75{ }^{\circ} \mathrm{C}$, treated with trypsin, resolved in 16\% polyacrylamide/7 M Urea gels and visualized using a PhosphoImager. In partial tryptic digests (indicated by $<$ and $<<$ ), increased peptide length retards band migration. $C$. The same reactions as in $B$, except trypsin digests were performed in presence of $2 \mathrm{M}$ Urea. 
be assessed by the accumulation of a radiolabelled $35 \mathrm{nt}$ product. In this assay, $40 \%$ less $\mathrm{G}^{717} \mathrm{D}$ mutant protein was used such that the amount of covalent complex formed at $\mathrm{t}=0$ was more comparable to reactions containing wild-type Top1. Aliquots, taken at the indicated time points were treated with trypsin in the presence of $2 \mathrm{M}$ urea (Fig. 4.5.B). In comparison with hTop1, the increased levels of covalent hTop $1 \mathrm{G}^{717} \mathrm{D}$-DNA complexes evident at $\mathrm{t}=0$, coincided with elevated levels of religated product (Fig. 4.5.B). Thus, consistent with steady state DNA cleavage assays, the $\mathrm{G}^{717} \mathrm{D}$ mutant failed to increase covalent enzyme-DNA intermediate stability. In the absence of CPT, elevated rates of DNA religation attended the increased rates of DNA cleavage. However, when the reaction products were subjected to trypsin digestion in the absence of urea (Fig. 4.5.C), additional bands of intermediate size were obtained with $\mathrm{hTop} 1 \mathrm{G}^{717} \mathrm{D}$ (arrow heads in Fig. 4.5.C). These bands were completely resolved as 14 nt-peptides in urea denatured tryptic digests (as in Fig. 4.5.B), and corresponded to 14 nt oligos covalently linked to peptides longer than 7 amino acid residues. These data suggest that the presence of Asp at position 717 alters the local architecture of the active site to limit trypsin digestion in the absence of urea. Indeed, similar results were obtained with the corresponding yeast Top $1 \mathrm{G}^{717} \mathrm{D}$ mutant enzyme, and in both cases, corresponded to the increased intrinsic CPT sensitivity of the mutant enzyme.

We next asked what effect duplex DNA downstream of the cleavage site, would have on active site structure. In the pre-annealed DNA religation assay (diagrammed in Fig. 4.6.A), the presence of the religation oligo prior to DNA cleavage by the $\mathrm{G}^{717} \mathrm{D}$ mutant altered the pattern of tryptic-DNA fragments obtained, such that the faster migrating band accumulated (Fig. 4.6.B). Moreover, these changes coincided with a 


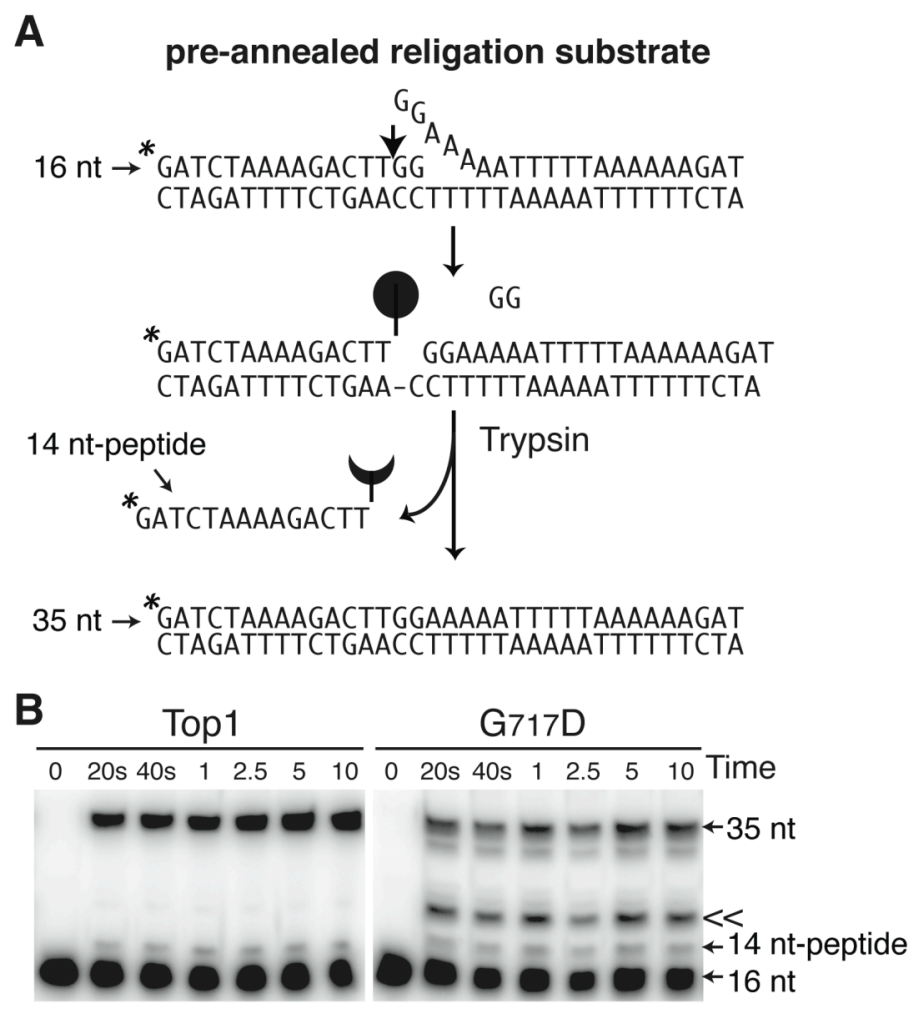

FIG. 4.6. The presence of duplex DNA downstream from the site of DNA scission reduces the rate of $h T 0 p 1 G^{717} D$-catalyzed DNA religation. $A$. In a pre-annealed religation assay, the $21 \mathrm{nt}$ religation oligonucleotide is annealed prior to the addition of enzyme. DNA cleavage by hTop1 liberates a GG dinucleotide, which allows religation to generate a 35 nt product. $B$. At $\mathrm{t}=0$, equal concentrations of hTop 1 and $h$ Top $1 G^{717} \mathrm{D}$ were added to pre-annealed religation assays and reaction aliquots were quenched with SDS at $75^{\circ} \mathrm{C}$ at the times indicated. Covalent DNA-Top1 complexes were hydrolyzed with trypsin and the reaction products resolved in $16 \%$ polyacrylamide/7M Urea gels and visualized with a PhosphorImager. Partial tryptic digests corresponding to those obtained in the post-annealed religation assay (Fig. 4.3.B) are indicated by $<<$. 
decreased rate of DNA religation (reduced amounts of the $35 \mathrm{nt}$ product over time, relative to that observed with wild-type hTop1). Thus, as with the yeast mutant enzyme (184), the presence of the religation substrate within the active site of $h T^{\circ o p} 1 \mathrm{G}^{717} \mathrm{D}$, but not wild-type hTop1, altered the rate of DNA religation and the architecture of the active site as probed by limited trypsin digests. A similar alteration in $\mathrm{G}^{717} \mathrm{D}$ enzyme active site architecture was revealed in tryptic digests of covalent complexes formed with the suicide substrate containing the 5' bridging phosphorothiolate (see Fig. 4.4). Thus, the structural changes revealed by the accumulation of the longer 14 nt-peptide in Fig. 4.6.B was also observed with a substrate comprising two intact DNA strands prior to DNA cleavage.

The relative migration of such peptide linked-DNA oligonucleotides in denaturing gels is proportional to the length of the peptide bound $(184,187)$. Based on comparisons of peptide-linked oligos obtained with $y \operatorname{Top} 1 \mathrm{G}^{721} \mathrm{D}$ and the relative positions of potential trypsin cleavage sites in close proximity to the active site Tyr residue, the identity of the peptide-linked oligos was readily determined. As shown in Fig. 4.7.B and C, when TPT is bound within the covalent Topo70-DNA complex, an $\alpha$-helix extends from the active site $\mathrm{Tyr}^{723}$ (magenta) to include $\mathrm{Gly}^{717}$ (yellow) and a rigid linker extends from the Top1 core at an oblique angle to the DNA. The Lys ${ }^{720}$ residue cleaved by trypsin to generate the canonical $14 \mathrm{nt}+7$ residue peptide is now positioned immediately adjacent to $\mathrm{Gly}^{717}$ on the same face of the $\alpha$-helix. In the absence of drug, $\mathrm{Gly}^{717}$ is found in a loop and the flexibility of the linker domain precludes structural determination. As Asp is predicted to have a higher propensity to form an $\alpha$-helical structure than Gly (178), we reasoned that the extension of this $\alpha$-helix in the absence of drug might preclude efficient trypsin 


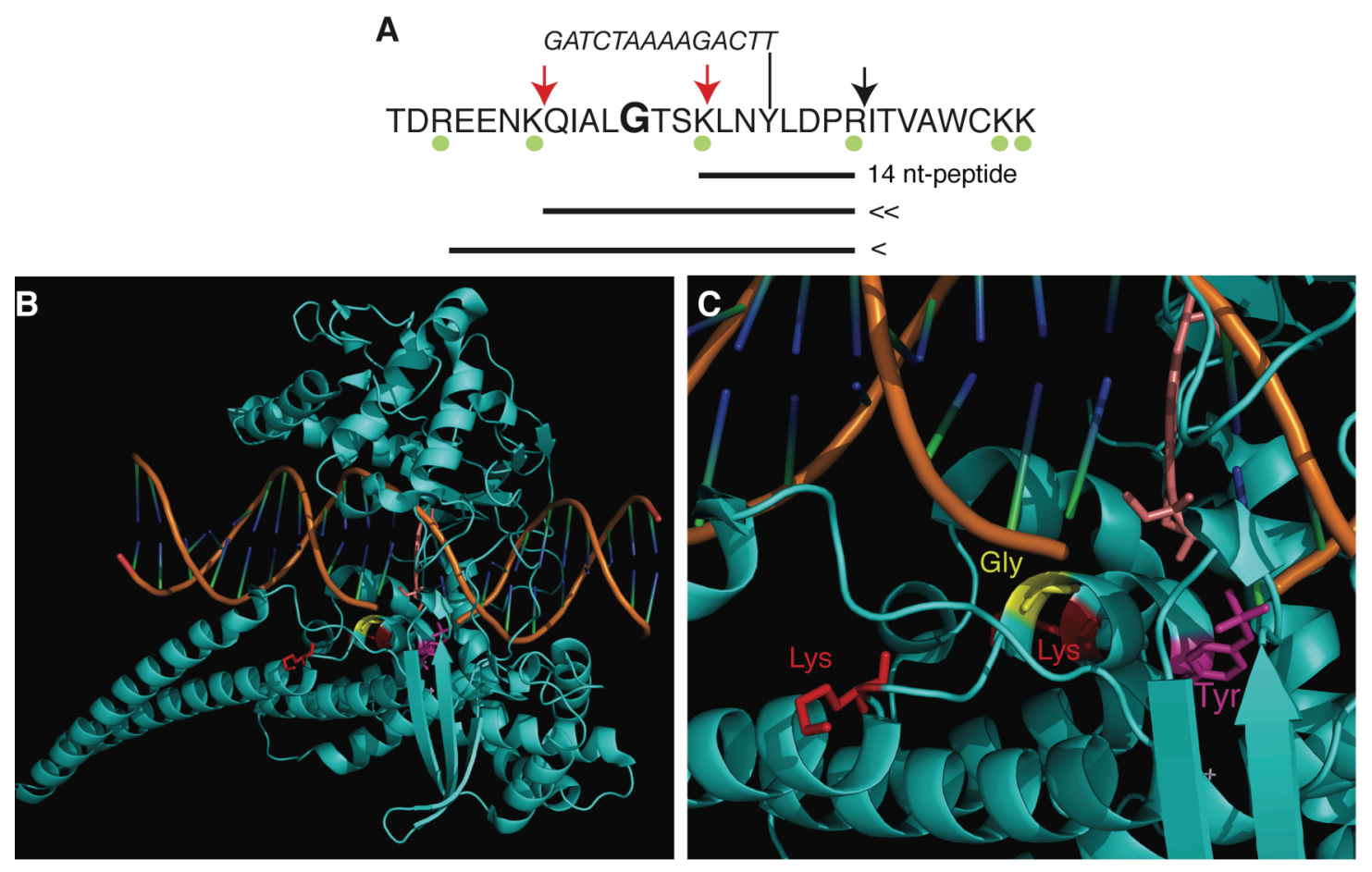

FIG. 4.7. Co-crystal structure of TPT bound to the covalent Topo70-DNA complex. $A$. Potential trypsin cleavage sites around the active site $\operatorname{Tyr}^{723}(\mathrm{Y})$ are marked with green dots. Trypsin cleavage after Arg (R marked with black arrow) and Lys ${ }^{720}$ (K marked with red arrow, 3 residues N-terminal to the active site Tyr) will generate a $14 \mathrm{nt}$ DNA linked to a 7 residue peptide via a phosphotyrosyl linkage (14 nt-peptide). Cleavage sites predicted to yield the $<$ and $<<$ DNA-peptide products observed in Fig. 4.3.B and 4.4.B are also indicated (Lys ${ }^{712}-\mathrm{K}$ red arrow for $<$; $\mathrm{Arg}^{708}-\mathrm{R}$ green dot for $<<$ ). The $\mathrm{Gly}^{717}$ residue mutated to Asp in hTop $1 \mathrm{G}^{717} \mathrm{D}$ is in large bold font. $B$ and $C$. Full view and close up ribbon diagrams of the co-crystal structure of Topo70 (light blue) covalently linked to DNA in the presence of TPT (pink), (PDB file 1K4T (59)). Orange traces the DNA phosphodiester backbone with green-blue sticks for bases. The active site Tyr is in magenta, Gly ${ }^{717}$ is in yellow, while Lys ${ }^{720}$ and Lys ${ }^{712}$ are in red. The figure was made with PyMol (DeLano Scientific, San Carlos CA). 
digestion at residue Lys ${ }^{720}$, thereby generating the longer 15 amino acid peptide bound to the $14 \mathrm{nt}$ DNA ( $<<$ in Figs. 4.5.C and 4.6.B). The presence of the religation oligo prior to DNA cleavage, also precluded efficient trypsin digestion of $\mathrm{hTop} 1 \mathrm{G}^{717} \mathrm{D}-\mathrm{DNA}$ complexes at the more $\mathrm{N}$-terminal $\mathrm{Lys}^{712}$. As this residue lies at the $\mathrm{C}$-terminal end of the linker domain, these data suggest that the alterations in DNA structure within the $\mathrm{G}^{717} \mathrm{D}$ mutant active site also impact protein structure. Together with the results obtained with yTop1, these data suggest conserved aspects of active site architecture impact the intrinsic CPT sensitivity of Top1.

\subsubsection{N-terminal and linker human/yeast Top1 chimeras}

Expression of $\mathrm{hTopG}^{717} \mathrm{D}$ failed to enhance CPT-induced toxicity in yeast, despite an apparent increase in mutant enzyme sensitivity to CPT in vitro. This surprising result prompted us to ask if the different phenotypes induced by conserved Gly to Asp mutations in yeast and human Top1 were due to the distinct structural features of these enzymes. For instance, while the N-termini of human and yeast Top1 are rich in acidic and basic residues, the N-terminal domain of yTop1 is $\sim 2 / 3$ the size of the corresponding domain of hTop1 (diagrammed in Fig. 4.8.A). This contrasts with the inverse relationship in linker length between the human and yeast enzymes (184). In fragment complementation experiments, polypeptides comprising the conserved core and Cterminal domains can reconstitute an active enzyme in vitro (39). However, the Nterminal domain has been shown to mediate Top1 interaction with other proteins, such TATA Binding Protein (TBP), nucleolin, SV40 large T antigen $(32,33,36)$, while linker 

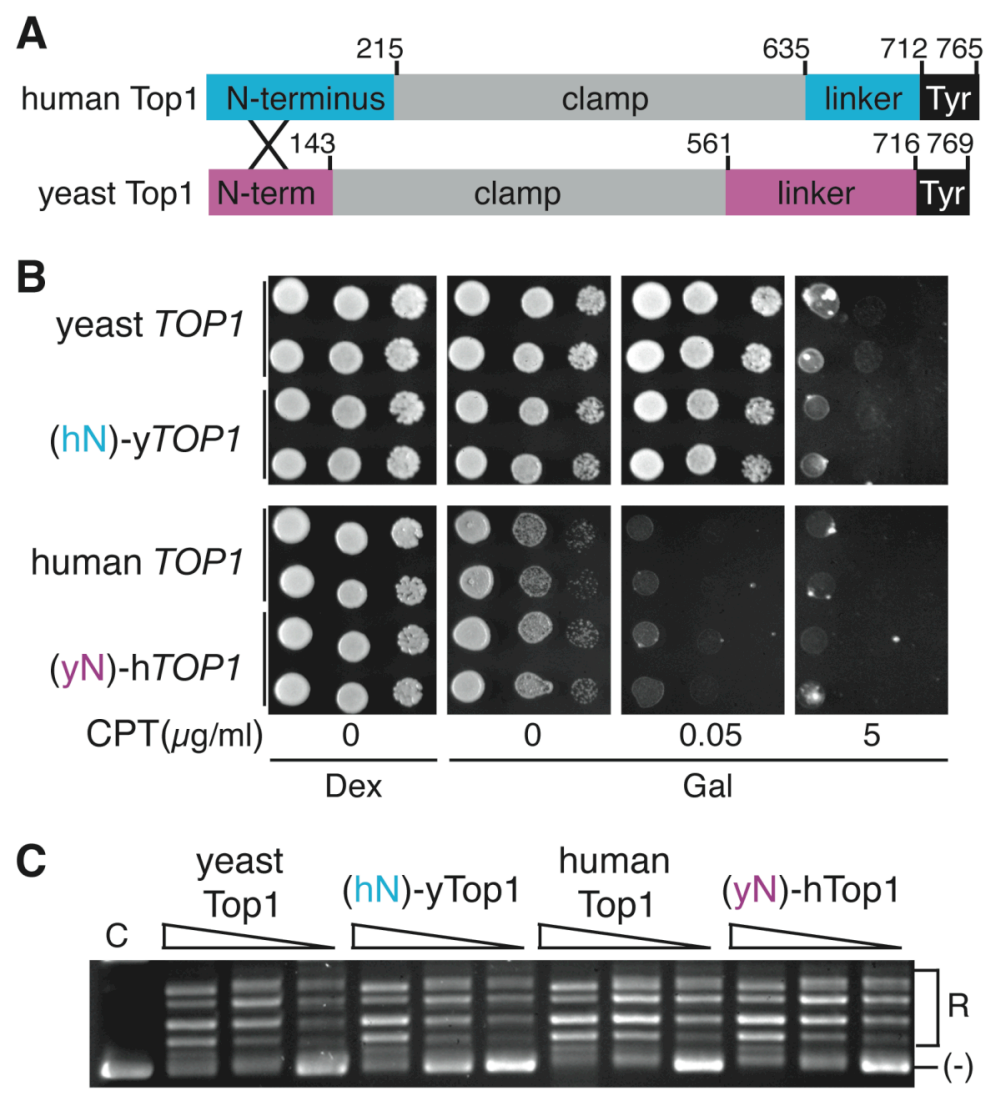

FIG. 4.8. Reciprocal swaps of the N-terminal domains of yeast and human Top1 do not alter enzyme activity or yeast cell sensitivity to CPT. $A$. Scheme of N-terminal residues exchanged between yeast and human Top1 via homologous recombination. The chimeric $\mathrm{N}$-terminal junctions were engineered at human residue 215 (for $\mathrm{hN}$ ) and yeast residue 143 (for $\mathrm{yN}$ ) to ensure that the conserved clamp domains were intact. (hN)yTOP1 encodes the yeast enzyme with a human N-terminal domain; (yN)-hTOP1 encodes human Top1 with the yeast N-terminus. B. Exponential cultures of top $1 \Delta$ cells, transformed with the indicated YCpGAL1-TOP1 constructs, were adjusted to an $A_{595}=$ 0.3 , serially 10 -fold diluted, and aliquots were spotted onto $\mathrm{SC}$-uracil agar containing 25 mM HEPES (pH 7.2), 0.1\% DMSO and either dextrose (Dex), galactose (Gal) or galactose plus the indicated concentration of CPT. The viability of two independent transformants was assessed following incubation at $30^{\circ} \mathrm{C}$. C. Equal concentrations of purified Top 1 proteins were serially 10 -fold diluted and incubated in a plasmid DNA relaxation assay. After $30 \mathrm{~min}$ at $30^{\circ} \mathrm{C}$, the reaction products were resolved by agarose gel electrophoresis and visualized with ethidium bromide. The relative positions of relaxed (R) and supercoiled (-) DNA topoisomers are indicated. C indicates DNA alone. 
flexibility appears to dictate enzyme sensitivity to CPT $(59,61,62,184)$. To address the function of these Top1 domains in regulating enzyme activity in vivo, homologous recombination [as previously described (185)] was used to generate reciprocal swaps of the N-terminal and linker domains of yTop1 and hTop1.

As diagrammed in Fig. 4.8.A and described in Table 4.1, a series of $\mathrm{N}$-terminal domain chimeras were engineered, including reciprocal swaps where (hN)-yTop1 contains human residues 1-191 fused to yeast residues 120-769 and (yN)-yTop1 contains yeast residues 1-119 fused to human residues 192-765. However, exchanging these domains had no effect on the pattern of in vivo CPT sensitivity in yeast. Cells expressing (hN)-yTop1 were sensitive to the same concentrations of CPT as cells expressing wildtype yTop1, while cells expressing either wild-type hTop1 or (yN)-hTop1 were sensitive to 10 -fold lower concentrations of drug (Fig. 4.8.B). The levels of chimeric proteins expressed and the specific activity of the purified proteins also mirrored that of the corresponding wild-type enzyme (Fig. 4.8.C and data not shown). Similar results were also obtained with $\mathrm{N}$-terminal chimeras containing different junctions upstream of the core domain (see Table 4.1 and data not shown). These findings eliminate the simple explanation that differences in yeast protein interactions with the $\mathrm{N}$-terminal domains of yeast or human Top1 alone could account for the differences in $h T o p 1 \mathrm{G}^{717} \mathrm{D} C \mathrm{CPT}$ sensitivity in vitro and in yeast cells.

We next engineered reciprocal exchanges of the linker domains. To accomplish this, the linker and C-terminus of each enzyme was replaced with the corresponding residues of the other. These chimeras were then used in reciprocal swaps to reintroduce the corresponding C-terminal domains, such that the (yL)-hTop1 chimera consisted of the 
human $\mathrm{N}$-terminal, core and $\mathrm{C}$-terminal domains, yet contained the yeast linker. The precise chimera junctions correspond to the conserved residues found at the $\mathrm{N}$ - and $\mathrm{C}$ terminal ends of the coiled coils of the linker, evident in the co-crystal structure of the drug bound covalent Topo70-DNA complex (59), i.e, hTop1 residues 635 and 712 and yTop1 residues 561 and 716 (for details see Table 4.1).

Replacing the shorter hTop1 linker with the longer yeast linker, in (yL)-hTop1, induced a surprising phenotype in yeast cells expressing this chimera, with no apparent alteration in enzyme activity in vitro. As shown in Fig. 4.9.B, galactose-induced expression of (yL)-hTop1 suppressed yeast cell growth, even in the absence of CPT. However, when the corresponding human N-terminal domain was introduced into this chimera [to generate (yN,yL)-hTop1], such that the conserved core and C-terminal domains were hTop1, while the divergent $\mathrm{N}$-terminal and linker domains were of yeast Top1 origin, this toxic phenotype was suppressed. Moreover, the cells expressing this (yN,yL)-hTop1 chimera were just as sensitive to CPT as cells expressing the wild-type human Top1. This was true whether the chimeras contained the $y \mathrm{~N}$ or $\mathrm{yN}^{120} \mathrm{~N}$-terminal fusion (Table 4.1, data not shown). In all cases, comparable levels of Top1 protein were expressed in these cells (data not shown). Also, as both $\mathrm{N}$ - terminal domains contain nuclear localization signals (127), it is unlikely that the observed phenotypic differences were due to changes in nuclear import. The specific enzyme activities of the purified chimeras were indistinguishable from that of wild-type hTop1 over a range of salt concentrations (Fig. 4.9.C). As shown in Fig. 4.10, at least 100-fold higher concentrations of CPT were required to induce similar levels of covalent yTop1- DNA intermediates as that observed for wild-type hTop1. These results are further consistent with the 

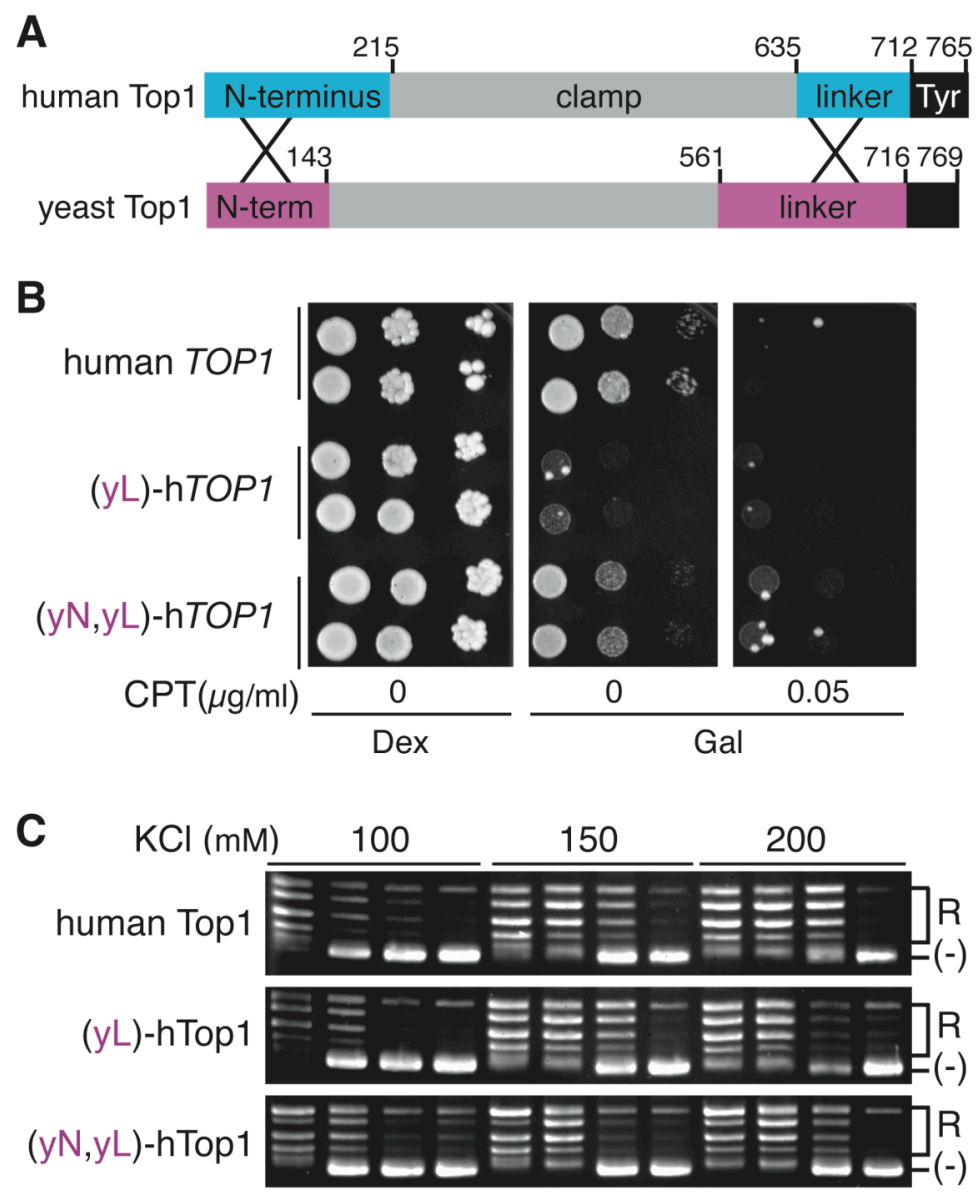

FIG. 4.9. The introduction of the yeast $\mathrm{N}$-terminal domain suppresses the lethal phenotype of a human Top1-yeast linker domain chimera. $A$. As in Fig. 6A, a scheme of the N-terminal and linker domains used to generate the indicated chimeras. Residues corresponding to the chimera junctions are as numbered. (hN-yL)-hTOP1 encode human Top1 with a yeast linker domain; (yN-yL)-hTOP1 encodes human Top1 with a yeast Nterminal and yeast linker domain. B. As in Fig. 4.6.B, serial dilutions of exponential cultures of top $1 \Delta$ cells, transformed with the indicated YCpGAL1-htop1constructs, were spotted onto SC -uracil agar containing $25 \mathrm{mM}$ HEPES (pH 7.2), 0.1\% DMSO and either dextrose (Dex), galactose (Gal) or galactose plus the indicated concentration of CPT and cell viability was assessed following incubation at $30^{\circ} \mathrm{C}$. C. Equal concentrations of purified hTop1 and chimeric proteins were serially 10-fold diluted and incubated in a plasmid DNA relaxation assay containing the indicated concentration of $\mathrm{KCl}$. After 30 min at $30{ }^{\circ} \mathrm{C}$, the reaction products were resolved by agarose gel electrophoresis and visualized with ethidium bromide. The relative positions of relaxed $(\mathrm{R})$ and supercoiled (-) DNA topoisomers are indicated. 


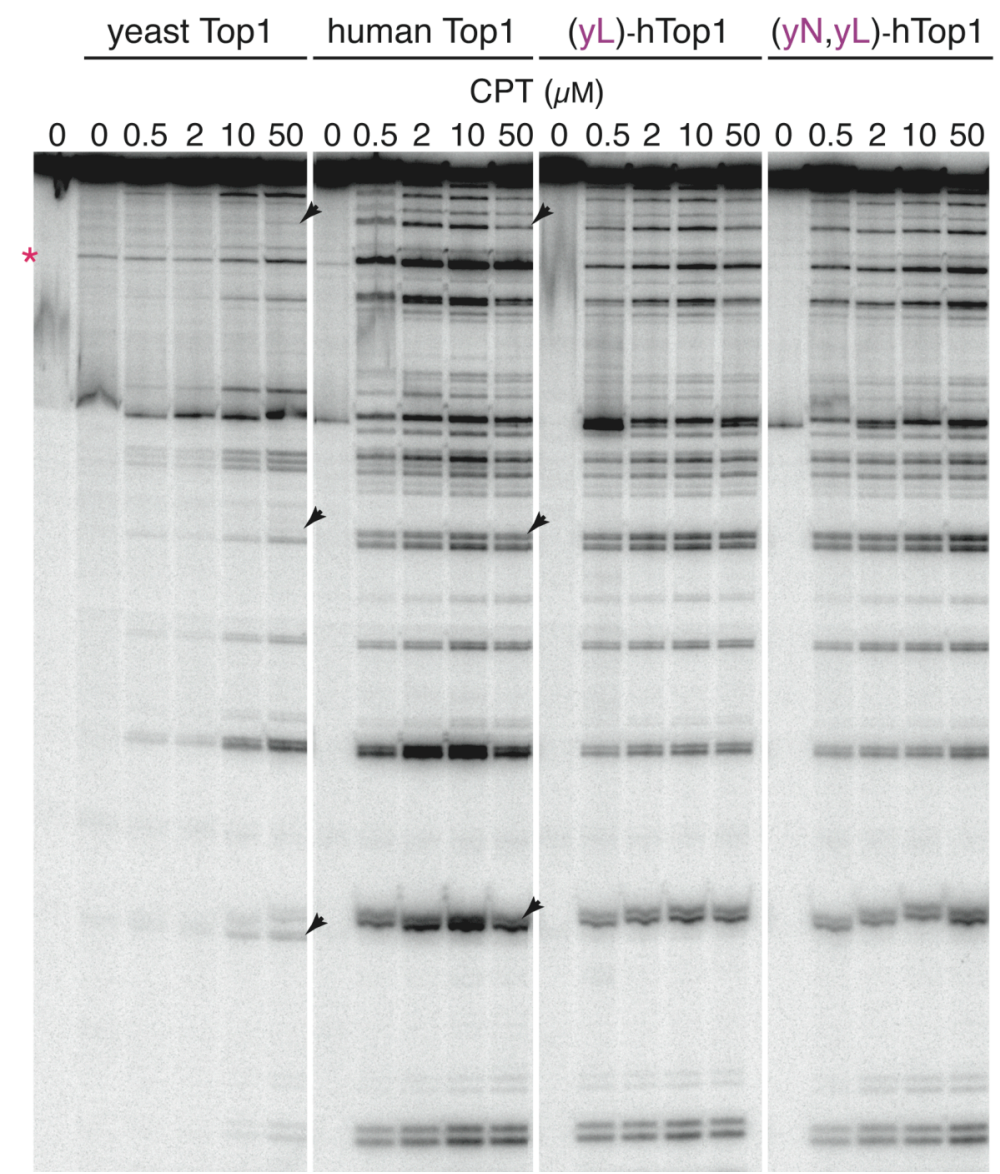

FIG. 4.10. CPT sensitivity and sequence specificity are dictated by the core and Cterminal domains of Top1. Equal concentrations of the purified Top1 proteins (described in Fig. 4.7.A) were incubated with a single $3{ }^{32} \mathrm{P}$-end labeled DNA substrate in a DNA cleavage reaction containing $0,0.5,2,10$ or $50 \mu \mathrm{M} \mathrm{CPT}$. After incubation for $10 \mathrm{~min}$ at $30{ }^{\circ} \mathrm{C}$, the covalent Top1-DNA complexes were trapped with SDS at $75{ }^{\circ} \mathrm{C}$ and treated with proteinase $\mathrm{K}$. The reaction products were resolved in $8 \%$ polyacrylamide/7M urea gels and visualized using a PhosphoImager. C is DNA alone and the asterisk (*) indicates a high affinity Top1 cleavage site. 
differences in in vivo CPT sensitivity seen in Fig. 4.8.B. Yet, independent of linker and/or N-terminal domain composition, the intrinsic CPT sensitivity of these hTop1 chimeras in vitro mirrored that of wild-type hTop1, not yTop1. Moreover, the subtle differences in sequence specificity between the yeast and human enzymes, as indicated by the arrow heads in Fig. 4.10, was also retained by the hTop1 chimeras.

Thus, the toxic phenotype of (yL)-hTop1 expressing cells was not due to any obvious alterations in enzyme binding to DNA, sequence specificity or shift in cleavagereligation in the absence of CPT. Indeed, as shown in Fig. 4.11, the growth defect induced by (yL)-hTop1 was independent of DNA cleavage, as galactose-induced expression of a catalytically inactive $(\mathrm{yL})-\mathrm{hTop} 1 \mathrm{Y}^{723} \mathrm{~F}$ mutant induced the same phenotype. Taken together, these findings support a model whereby the intrinsic CPT sensitivity of Top1 is dictated by the composition of the core and C-terminus, while the functional interaction of hTop1 with other proteins/ protein complexes in yeast is affected by linker composition. In the context of hTop1, the ability of the yeast N-terminal domain to suppress the growth defect induced by the yeast linker, further implies the coordinated function of these poorly conserved in regulating hTop1 interactions with other, as yet unidentified, proteins or protein complexes.

As the human linker is half the size of that of yeast Top1, with a proportionally larger N-terminal domain, we next assessed the consequence of mis-pairing the shorter human linker with the yeast enzyme (see Table 4.1). GAL1-promoted expression of this (hL)-yTop1 chimera induced a CPT resistant phenotype (Fig. 4.12.A), which corresponded to a $>10$-fold reduction in enzyme activity in a plasmid DNA relaxation assay in comparison with wild-type yTop1 (Fig. 4.12.B). Moreover, the optimal $\mathrm{KCl}$ 


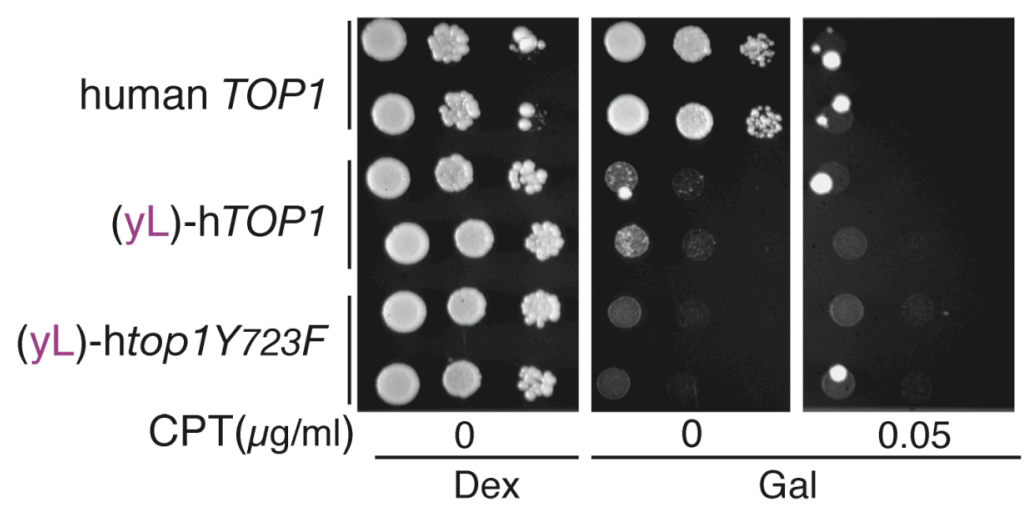

FIG. 4.11. Catalytically inactive hTop1 containing a yeast linker is toxic to yeast cells. Exponentially growing cultures of top $1 \Delta$ cells, transformed with the indicated YCpGAL1-top1 constructs, were adjusted to an $A_{595}=0.3$, serially 10 -fold diluted, and aliquots were spotted onto SC-uracil plates containing $25 \mathrm{mM}$ Hepes (pH 7.2), 0.1\% DMSO and either dextrose (Dex), galactose (Gal) or galactose plus $0.05 \mu \mathrm{g} / \mathrm{ml} \mathrm{CPT}$. Viability was assessed following incubation at $30{ }^{\circ} \mathrm{C}$. 

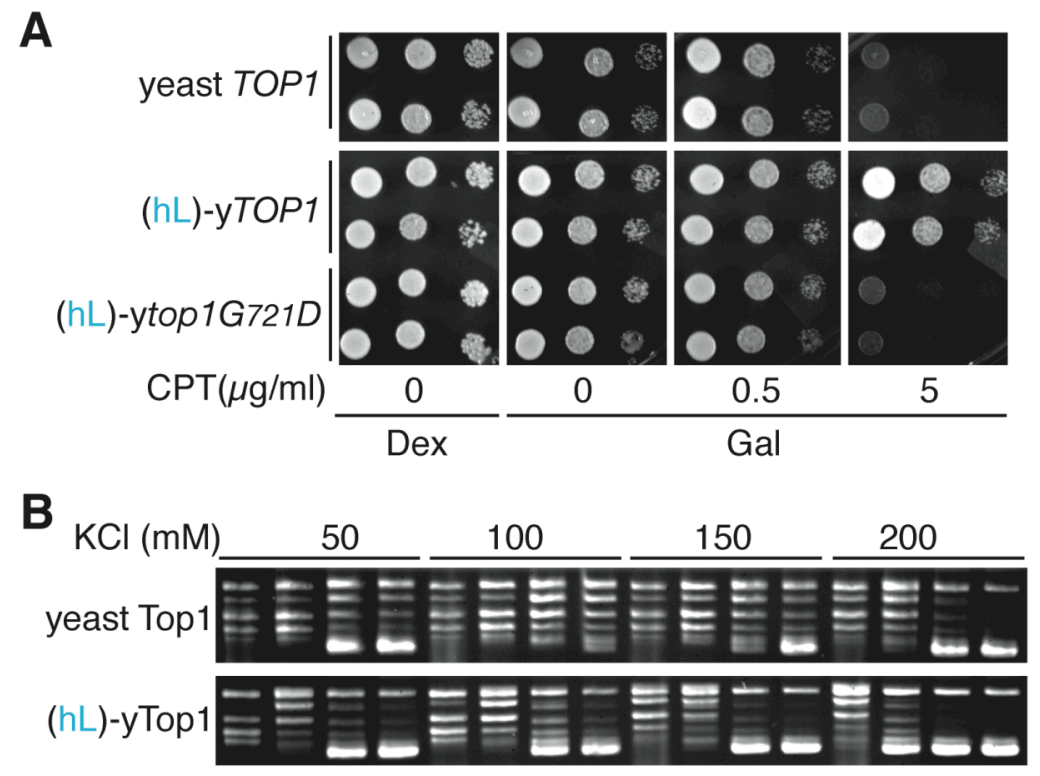

FIG. 4.12. Yeast Top $1 G^{721} D$ containing the human linker domain exhibits increased CPT sensitivity. A . Exponentially growing cultures of top $1 \Delta$ cells, transformed with the indicated YCpGAL1-TOP1 constructs, were adjusted to an $A_{595}=0.3$, serially 10 -fold diluted, and aliquots were spotted onto SC-uracil plates containing $25 \mathrm{mM}$ Hepes $(\mathrm{pH}$ 7.2), $0.1 \%$ DMSO and either dextrose (Dex), galactose (Gal) or galactose plus $0.05 \mu \mathrm{g} / \mathrm{ml}$ CPT. Viability was assessed following incubation at $30{ }^{\circ} \mathrm{C}$.

$B$. Extracts of galactose induced top $1 \Delta$ cells, expressing wild-type yTop 1 or (yN-hL) yTop1 from a YCpGAL-TOP vector, were corrected for protein concentration, serially 10 -fold diluted and incubated in a plasmid DNA relaxation assay containing the indicated concentration of $\mathrm{KCl}$. After $30 \mathrm{~min}$ at $30{ }^{\circ} \mathrm{C}$, the reaction products were resolved by agarose gel electrophoresis and visualized with ethidium bromide. The relative positions of relaxed and supercoiled DNA topoisomers are as in Fig. 4.9.C. 
concentration for plasmid DNA relaxation was lower for (hL)-yTop1, suggesting a reduction in DNA binding. When the yeast $\mathrm{Gly}^{721}$ residue (corresponds to human $\mathrm{Gly}^{717}$ ) was mutated to Asp in this chimeric enzyme $\left[(\mathrm{hL})-\mathrm{yTop} 1 \mathrm{G}^{721} \mathrm{D}\right]$, cell sensitivity to CPT sensitivity was enhanced, albeit not to the same level as with cells expressing the fully yTop $1 \mathrm{G}^{721} \mathrm{D}$ (Fig. 4.12.A, data not shown). These data demonstrate that mutation of the conserved Gly to Asp increases Top1 sensitivity to CPT, independent of the linker composition of the enzyme.

\subsection{DISCUSSION}

In eukaryotes, the unusual structural organization of the nuclear Top1 enzyme comprises a protein clamp, formed by a highly conserved core domain, that completely circumscribes duplex DNA $(12,27,59)$. The extended coiled-coil of the linker domain connects the core with another conserved C-terminal domain, which contains the active site tyrosine and is positioned against the clamp to complete the catalytic pocket of the enzyme. These nuclear enzymes also contain a highly charged $\mathrm{N}$-terminal domain, for which little structural information is available, but has been implicated in regulating the intracellular localization of Top1, Top1-protein interactions and the catalytic activity of Top1 in DNA relaxation assays $(34,37,127)$.

Questions concerning the relative movement of Top1 protein domains during enzyme catalysis and how these movements impact Top1 sensitivity to drugs that target this enzyme, such as CPT, have been of considerable interest. For instance, in co-crystal structures of human Topo70 with DNA, the tight packing of the DNA duplex within the 
pore of the protein clamp indicated considerable flexibility was necessary for Top1 to bind DNA $(12,27,59)$. Using molecular modeling, we designed a reversible disulfide bond that locked the Top1 clamp around duplex DNA, which prevented DNA strand rotation within the covalent enzyme-DNA intermediate (41). Thus, some breathing of the protein clamp is necessary for Top1 binding of DNA and for DNA strand rotation. The flexibility of the linker domain has also been implicated as a critical determinant of enzyme sensitivity to the CPT class of chemotherapeutics. The movement of this coiledcoil in covalent Topo70-DNA complexes precludes structural determination, while drug binding apparently restricts the orientation of the linker to an angle oblique to the DNA (59). Increasing the movement of this domain, either by physically uncoupling the Top1 linker and core domains in reconstitution experiments (61), or by mutation of $\mathrm{Ala}^{653}$ to Pro in hTop1 (62), decreases enzyme sensitivity to CPT. The concept of long range molecular interactions between the linker and the active site of the enzyme are further supported by two recent studies. First, the lethal phenotype induced by the human Top $1 \mathrm{~T}^{722} \mathrm{~A}$ mutant, which is defective in enzyme-catalyzed DNA religation, was suppressed by the increased linker flexibility of the $\mathrm{A}^{653} \mathrm{P}$ mutation (183). Second, we showed that substitution of $\mathrm{Gly}^{721}$ within the active site of yeast Top1 with residues estimated to have a higher propensity for $\alpha$ helix formation, induced alterations in enzyme active site architecture and increased yTop1 sensitivity to CPT (184). On the basis of structural considerations, where the extension of a short a helix within the active site of Topo70 to include the corresponding Gly ${ }^{717}$ residue of human Top1 was coincident with drug binding and a restriction of linker orientation (59), we posited that 
this conserved Gly provided a flexible hinge to facilitate the alterations in linker flexibility induced by CPT.

Here we show that mutation of $\mathrm{Gly}^{717}$ in $\mathrm{hTop} 1 \mathrm{G}^{717} \mathrm{D}$ also enhances enzyme sensitivity to CPT, which further supports a model whereby the flexible hinge provided by Gly allows for alterations in active site geometry and linker flexibility that attend CPT poisoning of hTop 1 . However, the inability of the $h T$ Top $1 \mathrm{G}^{717} \mathrm{D}$ to alter yeast cell sensitivity to CPT led us to consider the relative contribution of individual protein domains to Top1 sensitivity to CPT and enzyme function in vivo.

First, our studies demonstrate that, in the context of full length human Top1, the $\mathrm{G}^{717} \mathrm{D}$ mutation increased intrinsic enzyme sensitivity to CPT by selectively enhancing the rate of DNA cleavage. This effect on enzyme catalysis was only evident in assays using a variety of suicide substrates to uncouple Top1-catalyzed DNA cleavage from religation. Under steady state conditions with duplex DNA, an increase in covalent Top $1 \mathrm{G}^{717}$ D-DNA complexes was only evident in the presence of CPT. As DNA cleavage is, in part, the rate limiting step in enzyme catalysis (168), there was no apparent defect in the rate at which these covalent complexes were resolved. However, in the presence of CPT, the bound drug increases the stability of the covalent complex by reducing the rate of DNA religation $(59,180)$. Taken together, these data suggest the increased rate of DNA cleavage induced by the $\mathrm{G}^{717} \mathrm{D}$ mutant coupled with the decreased rate of DNA religation induced by drug binding, enhance CPT poisoning of $h T$ op $1 \mathrm{G}^{717} \mathrm{D}$, relative to that observed with wild-type hTop1.

This increase in CPT sensitivity also coincided with $\mathrm{G}^{717} \mathrm{D}$ mutant induced alterations in active site architecture as probed by trypsin digests of the covalent hTop1 
$\mathrm{G}^{717} \mathrm{D}$-DNA intermediates. These results contrasted with assays of wild-type hTop1, which yield the canonical 14 nt- 7 residue peptide diagrammed in Fig. 4.7.A. In this context, it is worth noting that when $\mathrm{hTop} 1 \mathrm{G}^{717} \mathrm{D}$ cleavage of DNA occurred in the absence of duplex DNA 3' to the site of scission (the post- annealed religation assay of Fig. 4.5), partial tryptic digests were obtained at two Lys residues that flank the $\mathrm{G}^{717} \mathrm{D}$ mutation, i.e., Lys $^{712}$ and Lys ${ }^{720}$. This stretch of amino acids constitutes the physical connection between the $\alpha$ helix that contains $\mathrm{Tyr}^{723}$ covalently attached to DNA and the C-terminal end of the coiled-coil of the linker domain at Lys ${ }^{712}$ (59). In the absence of CPT, the $\alpha$ helix does not extend to include $\mathrm{Gly}^{717}$. However, when a CPT analog is bound in the Topo70-DNA complex, this $\alpha$ helical structure is no extended to include $\mathrm{Gly}^{717}$ and Leu ${ }^{716}$ (59). Since Asp is predicted to have a higher propensity to form an $\alpha$ helix than Gly (178), the extension of this $\alpha$ helical structure in the $\mathrm{G}^{717} \mathrm{D}$ mutant, in the absence of CPT, would position Asp three residues N-terminal to $\mathrm{Lys}^{720}$ and on the same face of the $\alpha$ helix. Such a structural transition might preclude efficient trypsin digestion in the absence of urea denaturation and possibly enhance CPT poisoning of Top1 by stabilizing an active site geometry that facilitates drug binding. In the presence of a $2 \mathrm{nt}$ flap and downstream duplex DNA prior to mutant $\mathrm{G}^{717} \mathrm{D}$ cleavage of the suicide DNA oligo (in the pre-annealed religation assay of Fig. 4.6), only trypsin digestion of Lys ${ }^{720}$ was affected by urea denaturation; no defect in proteolytic cleavage of the C-terminal end of the linker $\left(\mathrm{Lys}^{712}\right)$ was detected. These data suggest that in the presence of duplex DNA 3' to the site of scission, the structure of the C-terminal end of the $\mathrm{G}^{717} \mathrm{D}$ mutant linker is distinct from that observed in the absence of DNA. Arguably, the presence of the $2 \mathrm{nt}$ flap in these assays may constitute a typical DNA substrate for hTop1. However, as 
similar results were obtained with the covalent $h T o p 1 \mathrm{G}^{717} \mathrm{D}-\mathrm{DNA}$ complexes formed with the duplex suicide substrate containing a 5 ' bridging phosphorothiolate at the site of scission (Fig. 4.4), the alterations in $\mathrm{G}^{717} \mathrm{D}$ mutant active site architecture revealed by trypsin digestion were not an artifact of flap-induced distortions in the DNA. Rather they reveal distinct differences in the catalytic pocket of covalent hTop1-DNA complexes induced by the $\mathrm{G}^{717} \mathrm{D}$ mutation.

Second, our analyses of yeast /human Top1 chimera activity in vitro and in yeast indicate that in the context of full length enzymes, comprising an N-terminal, core, linker and C-terminal domain, the intrinsic CPT sensitivity is dictated by the composition of the core and C-terminus. Independent of the origin of the $\mathrm{N}$-terminal and/or linker domains (yeast or human), enzymes consisting of the human core and C-terminal domains exhibited comparable specific activity in plasmid relaxation assays and CPT sensitivity in DNA cleavage assays. However, distinct patterns of enzyme activity were evident in top $1 \triangle$ yeast cells expressing these various chimeras from $G A L 1$-promoted constructs. Reciprocal swaps of the highly charged yeast and human N-termini (at a juncture corresponding to human residue 192 or yeast residue 120), did not impact cell viability or the relative levels of cell sensitivity to CPT. Thus, any Top1-protein interactions mediated solely by these domains do not affect the extent of CPT-induced toxicity in these cells. Human Top1 residues 201-206 have also been implicated as playing a critical role in enzyme catalysis (37). However, fusing human Top1 residues 1-191 or 1-209 with the corresponding C-terminal residues of yTop1 failed to alter the CPT sensitivity of cells expressing these chimeras, relative to cells expressing wild-type hTop1 (Fig. 4.8 and data not shown). These data suggest that the critical function of these human residues 
(KWKWWEEE) in enzyme catalysis is retained in the corresponding residues of yeast Top1 (EYKWWEKE).

In contrast, the introduction of the longer yeast linker domain in hTop1 profoundly altered enzyme function in vivo. Indeed, the toxicity induced by overexpression of (yL)-hTop1 chimeras, containing the yeast linker, was independent of DNA cleavage. Yet in vitro, the CPT sensitivity and specific activity of the (yL)-hTop1 chimera was similar to that of hTop1. These findings suggest that the presence of the yeast linker may promote protein-protein interactions that alter the association of hTop1 with other cellular complexes in yeast, which adversely impact cell growth. The context of these associations and whether such complexes are DNA bound have yet to be addressed. However, the ability of the complementary yeast $\mathrm{N}$-terminal domain to suppress the toxicity induced by the presence of the yeast linker in hTop1 suggests a negative role for the $\mathrm{N}$-terminus in suppressing these interactions. Moreover, these data support a functional interaction between these two domains. Whether this is due to the direct physical association of these domains or indirect interactions mediated by DNA /other proteins has yet to be determined. In this context, it is noteworthy that the Nterminal domain, which has defied structural determination is absent from the human Top1 enzyme that is targeted to the mitochondria (182). Thus, the function of the Nterminal domain appears to be restricted to nuclear-Top1 interactions. 


\section{CHAPTER 5: IDENTIFICATION OF PROTEINS INTERACTING WITH TOP1 LINKER AND C-TERMINAL DOMAIN}

\subsection{INTRODUCTION}

Eukaryotic DNA Topoisomerase I (Top1) is a highly conserved enzyme that plays a role in the alteration of DNA topology during processes such as replication, transcription, recombination and chromosomal condensation. This enzyme consists of four domains: 1) an N-terminal domain that is not required for in vitro activity, but has been shown to interact with other proteins; 2) a core domain that forms a clamp circumscribing duplex DNA and contains all the catalytic residues except the catalytic $\operatorname{Tyr}\left(\operatorname{Tyr}^{723}\right.$ in human Top1); 3) a linker that forms a coiled coil extending away from the body of the enzyme, and 4) a C-terminal domain that is connected to the core through the linker and contains the catalytic Tyr responsible for cleavage $(3,4,39)$.

Even though this enzyme is highly conserved from budding yeast to humans, there are regions that deviate both in size and amino acid sequence. The core and Cterminal domains are the most conserved regions of Top1. An active enzyme can be reconstituted by combining individually expressed core and C-terminal domain polypeptides, indicating that these two domains are the minimal requirement for Top1 relaxation activity (39).

The N-terminus and linker domains are the most divergent regions of the enzyme. The yeast Top1 N-terminus is much smaller than that of human Top1, although both contain a high percentage of charged residues. Unfortunately no crystallographic data are 
available to establish the position of the N-terminus relative to the rest of the protein (12). In contrast to the N-terminus, the human linker is much smaller than that of yeast Top1. Crystallographic data of the $70 \mathrm{kD}$ C-terminal region of human Top1 and molecular modeling of the yeast Top1 linker indicate that this domain is structurally conserved, forming an extended coiled coil ( $\sim 56 \AA$ for human Top1; $\sim 110 \AA$ for yeast Top1). There are few contacts between the linker and the body of the enzyme giving this domain a high degree of flexibility $(12,59)$. No protein binding sites have been mapped to the linker domain.

Top1 does interact with various proteins and protein complexes, including the transcription initiation and elongation machinery, TATA Binding Protein (TBP), p53, nucleolin, SV40 large T antigen, a RING finger/RS rich protein named topors, which functions as a SUMO/Ub dual E3 ligase, and the spliceosomal protein, SF2/ASF (34, $100,127,133,137)$. Top1 has also been functionally linked to (138), or co-localized and co-purified with RNA polymerase I $(128,139)$. All of the above mentioned interactions occur through the N-terminal Top1 domain and the binding motifs within Top1 have been defined for nucleolin and SF2/ASF (34, 100, 127, 133, 137).

Our studies of chimeric proteins, in which human Top1 was engineered with a yeast linker, showed that this protein is toxic to yeast cells when overexpressed from a GAL1 promoter (See chapter 4). No differences in specific enzyme activity were detected between wild-type human Top1 and this chimera. Moreover, in DNA cleavage assays, where the steady state levels of covalent Top1-DNA intermediates were assessed in the presence of the Top1 poison CPT, no alteration in the CPT sensitivity or sequence specificity of the stabilized covalent complexes formed were detected. The surprising 
finding was that when the human Top1 N-terminus of this chimera was replaced with the yeast N-terminus, this protein was no longer toxic when expressed from the GAL1 promoter and the cells were sensitive to same level of CPT as cells expressing wild type human Top1, indicating a functional interaction between the $\mathrm{N}$-terminus and the linker domain. This idea is supported by the experiments of Lisby et al. (37), which suggest that the N-terminus interacts with DNA downstream of the cleavage site, the same segment of DNA that the linker has been shown to interact with in the crystallographic data of Topo70 non-covalently linked to a 22 basepair DNA duplex (12). More surprising was the fact that when an inactive chimeric protein of human Top1 containing the yeast linker ((yL)-hTop1) was overexpressed in yeast cells, it was still toxic, demonstrating that the toxicity is not due to the catalytic activity of the enzyme, but rather a loss or gain of function dictated by the linker domain.

To test this hypothesis, a two-hybrid screen was performed to identify specific proteins interacting with the linker domain, and then determining whether any differences exist in the binding of these proteins to the yeast and human linker. An additional reason for investigating potential binding partners of the linker, was the fact that while both the $\mathrm{hTop} 1 \mathrm{G}^{717} \mathrm{D}$ and the corresponding yeast mutant, $\mathrm{yTop} 1 \mathrm{G}^{721} \mathrm{D}$ were hypersensitive to the anticancer drug CPT in vitro, only the yeast mutant enzyme enhanced yeast cell sensitivity to CPT (See chapter 3 and 4). These data suggest distinct functional interactions of yeast and human Top1 with protein complexes in yeast cells. In chapter 4, we demonstrated that exchanging only the $\mathrm{N}$-terminal domain of yeast and human Top1 did not alter cell sensitivity to CPT, suggesting that it is either the linker or the combination of the linker and $\mathrm{N}$-terminus that dictate these functional interactions. 


\subsection{EXPERIMENTAL PROCEDURES}

\subsubsection{Chemicals, yeast strains and plasmids}

S. cerevisiae strain L40 (MATa, his34200trp1-901 leu2-3112 ade2

LYS2::4lexAop-HIS3 URA3::(lexAop-lacZ) GAL4), the Hybrid hunter kit, Zeocin ${ }^{\mathrm{Tm}}$ and AntiLex A antibody were purchased from Invitrogen. Plasmid pACT and Escherichia coli (E.coli) strain BNN132 containing the cre gene (188) were bought from ATCC. 3Aminotriazol was purchased from Sigma and X-gal from Promega.

\subsubsection{Construction of bait}

Bait proteins were constitutively expressed from the $A D H$ promoter on a $2 \mu$ Zeocin $^{+}$plasmid, (pHybLex/Zeo) and contained the LexA carboxy-terminal oligomerization region that contributes to operator occupancy by LexA derivatives (189). Two yeast bait constructs were generated by PCR amplification of yeast TOPI DNA sequences encoding the linker and $\mathrm{C}$-terminal domains (Link/C). The following forward primers were used to amplify yTop $1^{\mathrm{Link} / \mathrm{C}-1}$ and yTop $1^{\mathrm{Link} / \mathrm{C}-2}$ : $\operatorname{primer}\left(y \operatorname{Top} 1^{\mathrm{Link} / \mathrm{C}-1}\right) 5^{\prime}-$ ATCCGGAATTCACTGTCACGAAGGGGCATGCAC and primer (yTop $1^{\text {Link/C-2 }}$ ) 5'ATCCGGAATTCAAAGGATCTGTCGCAGAGAA. The same reverse primer was used for both amplifications: primer 5'- GATCGAGCTCTTAAAACCTCCAATTTTCATC. The forward primers contained an EcoRI and the reverse primer a SacI cleavage site (underlined) near the 5 '-ends to allow subcloning into $\mathrm{pHybLex} / \mathrm{Zeo}$.

To generate the human Top1 bait corresponding to $\mathrm{yTop} 1^{\mathrm{Link} / \mathrm{C}-1}$, human TOP1 sequences encoding the linker and C-terminus $\left(\mathrm{hTop} 1^{\mathrm{Link} / \mathrm{C}}\right)$ were PCR amplified using 
primers containing either a Sac1 or Kpn1 site near the 5'-ends for subcloning into pHybLex/Zeo: Forward primer 5'- TGAGAGCTCGCACCACCAAAAACTTTTGAG and reverse primer 5'- TACGGTACCCTAAAACTCATAGTCTTCATCAG. The amplified DNAs were cleaved and ligated into $\mathrm{pHybLex/Zeo} \mathrm{to} \mathrm{generate} \mathrm{pHybLx} / \mathrm{Zeo}-$

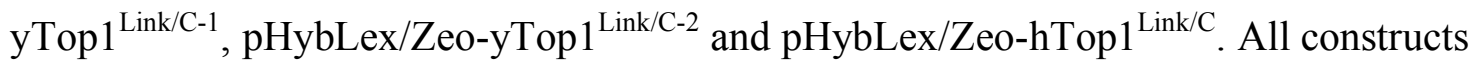
were confirmed by DNA sequencing and protein expression and integrity in yeast cells assessed by immunoblotting. Briefly, whole cell extracts were prepared by breaking open yeast cells using cracking buffer ( $8 \mathrm{M}$ Urea, 5\% SDS, $40 \mathrm{mM}$ Tris- $\mathrm{HCl}$ (pH 6.8), $0.1 \mathrm{mM}$ EDTA $1 \% \beta$-mercaptoethanol, $0.4 \% \mathrm{mg} / \mathrm{ml}$ bromophenol blue) and cold glass beads. Cell/glass bead suspension was incubated at $70{ }^{\circ} \mathrm{C}$ for 10 minutes and then vortexed for 1 minute. $30 \mu 1$ of the whole cell extract was used for immunoblotting. Proteins were resolved in a 4-12\% BisTris gel (Invitrogen), transferred onto activated polyvinylidene difluoride membrane (PerkinElmer Life Sciences), immunostained with the anti-LexA antibody (Invitrogen) and visualized by chemiluminescence.

\subsubsection{Automatic subcloning of plasmid library}

The $\lambda$-ACT library was converted into a plasmid library by Cre-LoxP recombination. $\lambda$-ACT, a Saccharomyces cerevisiae cDNA library in phage $\lambda$, was purchased from ATCC. The cDNAs were made using standard methods outlined by Durfee et al. 1993 and ligated into the Xho1 site on the $\lambda$-ACT arms (190). The cDNA insert and surrounding sequences are flanked by two LoxP sites. The conversion of $\lambda$ ACT into plasmid (pACT) was accomplished by incubating $10^{9}$ phage with a $1 \mathrm{ml}$ overnight culture of BNN132 cells in $10 \mathrm{mM} \mathrm{MgCl}_{2}$ for 30 min at $30^{\circ} \mathrm{C}$ without shaking. 
E.coli strain BNN132 has been engineered to constitutively express the Cre recombinase such that site-specific recombination by Cre at the LoxP sites would generate pACT plasmid harboring random yeast cDNAs that is N-terminally HA-tagged and located downstream of the sequence encoding the GAL4 activation domain (188). Two milliliters of LB was added and cells were incubated with shaking for $1 \mathrm{~h}$ at $30^{\circ} \mathrm{C}$. Cells were then plated on ten $150 \mathrm{~mm}$ LB-plates with $50 \mu \mathrm{g} / \mathrm{ml}$ ampicillin and incubated at $37^{\circ} \mathrm{C}$ overnight. $A p^{r}$ cells were scraped from these plates, added to 3 liters of terrific broth with ampicillin and grown overnight. Plasmids were isolated used a Quigen maxiprep kit.

\subsubsection{Screening for yeast Top $1^{\mathrm{Link} / \mathrm{C}-1}$ interactors}

Library screening was performed according to the Invitrogen Hybrid hunter protocol. Briefly, the bait strain, L40-yTop $1^{\text {Link/C-1 }}$ was generated by transforming strain L40 with $\mathrm{pHybLex} / \mathrm{Zeo}-\mathrm{yTop} 1^{\mathrm{Link} / \mathrm{C}-1}$, which contains the Zeocin resistance gene, by treatment with lithium acetate and heat shock in presence of DMSO. Transformants were selected and maintained on yeast extract-peptone-dextrose medium containing $0.1 \%$ adenine (YPAD) and $300 \mu \mathrm{g} / \mathrm{ml}$ Zeocin.

For the two-hybrid screen, $5 \times 10^{8}$ L40-yTop ${ }^{\text {Link/C-1 }}$ cells were transformed with 500 $\mu \mathrm{g}$ library plasmid DNA in a $1 \mathrm{~L}$ volume using lithium acetate, and heat shock $\left(42{ }^{\circ} \mathrm{C}\right)$ in the presence of DMSO. The cells were the incubated in YPAD at $30{ }^{\circ} \mathrm{C}$ for $1 \mathrm{~h}$ with constant shaking, pelleted and resuspended in YC-LU Z300 (synthetic medium lacking leucine and uracil and supplemented with $300 \mu \mathrm{g} / \mathrm{ml}$ Zeocin) and incubated, with shaking, at $30{ }^{\circ} \mathrm{C}$ for $16 \mathrm{~h}$. 
To confirm that sufficient numbers of transformants were screened, $1 \times 10^{-5}$ and $1 \times 10^{-6}$ dilutions, of the transformed cells, were plated on YC-LU Z300 to gauge transformation efficiency. To screen for interactions that restore histidine prototrophy, $5 \mu 1,10 \mu 1,25 \mu 1$ and $50 \mu 1$ of cells from the culture were plated on YC-LHUK Z300 (YC Z300 lacking leucine, histidine, uracil and lysine). All plates were incubated at $30{ }^{\circ} \mathrm{C}$ for 3-5 days.

A secondary screen was performed on the $\mathrm{His}^{+}$colonies to assess expression of the reporter gene, lacZ, using a X-gal filter assay as described in the Hybrid hunter protocol. Nitrocellulose filters sized to fit a $100 \mathrm{~mm}$ plate were laid on top of the $\mathrm{His}^{+}$ colonies grown, removed and frozen in liquid nitrogen. After the filter was thawed at room temperature, it was incubated with $1.5 \mathrm{ml} \mathrm{Z}$-buffer $\left(60 \mathrm{mM} \mathrm{Na}_{2} \mathrm{HPO}_{4}, 40 \mathrm{mM}\right.$ $\mathrm{NaH}_{2} \mathrm{PO}_{4}, 10 \mathrm{mM} \mathrm{KCl}, 1 \mathrm{mM} \mathrm{MgSO}$, $\mathrm{pH} 7.0$ ) and $30 \mu \mathrm{l}$ of $50 \mathrm{mg} / \mathrm{ml} \mathrm{X}$-gal in a Petri dish, covered with foil and incubated at $30{ }^{\circ} \mathrm{C}$ for 30 minutes. As expression of the lacZ gene product, $\beta$-galactosidase, is driven by binding of the LexA DNA binding domain (DBD) and recruitment of the Gal4 Activation domain (AD), a positive interaction is evident by the expression of $\beta$-galactosidase, which metabolizes X-gal to an insoluble blue product (5,5'-dibromo-4,4'-dichloro-indigo).

\subsubsection{Isolation of plasmid from yeast cells}

To identify yeast gene products which induces histidine prototrophy and $\beta$ galactosidase expression, plasmids were isolated from $\mathrm{His}^{+}$, blue colonies amplified in E.coli and the cDNA insert were sequenced. Briefly, a $5 \mathrm{ml}$ culture of each colony was grown overnight to an $\mathrm{OD}_{600}$ of 1 . The cells were pelleted and resuspended in $500 \mu 11$ of 
M sorbitol/20 mM EDTA. $30 \mu \mathrm{l} 20 \mathrm{~T}$ zymolase ( $5 \mathrm{mg} / \mathrm{ml}$ in $1 \mathrm{M}$ sorbitol) was added and suspension was incubated at $37{ }^{\circ} \mathrm{C}$ for 30 minutes. Cells were again pelleted, the plasmid DNA was extracted using using a Qiagen miniprep kit, transformed into E.coli and isolated from ampicillin-resistant transformants.

The cDNA inserts were sequenced using the following sequencing primer: 5 , GCTTACCCATAACGATGTTCCA. The DNA sequences obtained were then used to query the Saccharomyces Genome Database (http://www.yeastgenome.org/) to establish the identity of individual cDNAs.

\subsubsection{Confirmation of interaction, elimination of false positives and testing interaction with human Top1 linker and C-terminal domain}

To test for false positives and determine if the interactors found interacting with yTop $1^{\text {Link/C-1 }}$ also interact with hTop $1^{\text {Link/C }}$, L40 cells were transformed with pHybLex/Zeo-yTop $1^{\text {Link/C-1 }}$, pHybLex/Zeo-hTop $1^{\text {Link/C }}$ or pHybLex/Zeo. Each strain was co-transfomed with a library plasmid isolated from the screen and plated on YC-LU Z300. After 3 days of incubation at $30^{\circ} \mathrm{C}, 5$ colonies from each plate were diluted in TE, adjusted to $\mathrm{OD}_{600}=0.3$ and streaked onto YC-LU Z300, YC-LUHK Z300 and YCLUHK Z300 with $3 \mathrm{mM}$ 3-Aminotriazole (3-AT) plates. 3-AT is a competitive inhibitor of imidazole-glycerolphosphate dehydratase, the product of the HIS3 gene. The addition of 3-AT allows discrimination between weak and strong interactors, as 3-AT will inhibit low levels of dehydratase which will result in no growth on histidine-deficient medium. 


\subsubsection{Detection of physical interaction between $y$ Top $1^{\operatorname{Link} / \mathrm{C}-1}$ and proteins identified in two-hybrid screen using immunoprecipitations}

L40 cells were co-transformed with $\mathrm{pHybLex} /$ Zeo-yTop $1^{\text {Link/C-1 }}$ and a library plasmid isolated from the two-hybrid screen. These cells were grown in YC-LU Z300 media until they reached an $\mathrm{OD}_{600}=1$, washed in cold $\mathrm{dH}_{2} \mathrm{O}$ and resuspended in $\mathrm{TG}$ buffer (50 mM Tris- $\mathrm{HCl}, 150 \mathrm{mM} \mathrm{NaCl}, \mathrm{pH} 7.5,1 \%$ Triton $\mathrm{X}-100$ and 10\% glycerol), 2 $\mathrm{ml} / \mathrm{g}$ of wet cells. For $5 \mathrm{ml}$ of TG buffer also add $50 \mu \mathrm{l}$ Phosphatase inhibitor cocktail I (Sigma), $50 \mu 1$ Phosphatase inhibitor cocktail II (Sigma) and one protease inhibitor cocktail tablet (Complete Mini, Roche). Cells were frozen at $-80{ }^{\circ} \mathrm{C}$ overnight, transferred to eppindorf tubes containing cold glass beads and vortexed 10 times, one minute each and incubating the tubes on ice between each minute. Cell suspension was centrifuged at $4^{\circ} \mathrm{C}$ for 20 minutes and supernatant transferred to new tubes. Protein concentration was determined using a Biorad protein assay. $500 \mu 1$ cell extract containing equal protein concentration was added to $20 \mu \mathrm{l}$ protein A/G PLUS agarose beads (Santa Cruz Biotechnology) and rocked for $1 \mathrm{~h}$. Suspension was centrifuged and supernatant transferred to new tubes. $0.4 \mathrm{mg} / \mathrm{ml}$ anti-LexA antibody and $30 \mu 1$ protein A/G PLUS agarose beads were added to the supernatant and rocked overnight at $4{ }^{\circ} \mathrm{C}$. Sample was centrifuged and supernatant removed. The beads were washed 5 times with TG buffer containing phosphatase inhibitors, protease inhibitor and $0.05 \%$ sodium dodecyl sulphate (SDS). Beads were resuspended in $50 \mu \mathrm{l}$ SDS sample buffer (60 mM Tris- $\mathrm{HCl}$ (pH 6.8), 1.7\% SDS, 5\% glycerol, 0.1 M dithiothreitol, $0.002 \%$ Bromophenol blue), boiled for 5 minutes and centrifuged. Proteins in the supernatant were resolved in 4-12\% BisTris gels (Invitrogen), transferred onto activated polyvinylidene difluoride membranes 
(PerkinElmer Life Sciences), immunostained with the anti-HA anitibody (Roche) and visualized by chemiluminescence.

\subsection{RESULTS}

The two-hybrid system exploits the fact that transcription factors consist of two domains, a DNA binding domain (DBD) and an activation domain (AD), and both need to be present at the promoter region for transcriptional activation. For this screen two hybrid proteins were constructed. The first is a Lex DBD/Bait fusion and the second a Gal4 AD/Prey fusion. These two proteins are encoded by separate plasmids and both proteins are constitutively expressed from the $A D H$ promoter (Fig. 5.1).

To perform the screen, both plasmids are transformed into a yeast strain that contains two reporter genes, lacZ and an auxotrophic marker, HIS3. The regulatory region for both reporter genes contain the LexA DNA binding sites that acts as upstream activating sequences (UAS) in yeast. If the bait protein interacts with the prey, the activation domain will be recruited to the DNA binding domain to reconstitute a functional transcription factor, resulting in the expression of the reporter genes (Fig. 5.2).

The goal of this experiment was to determine what proteins, if any, specifically interact with the linker and C-terminal domain of yeast Top1 (yTop $1^{\mathrm{Link} / \mathrm{C}}$ ) and if any of

these interactions were altered in the context of the human Top $1^{\mathrm{Link} / \mathrm{C}}$. Therefore, we first used the yeast Top1 linker and C-terminal domains to avoid heterologous protein expression in yeast cells (Fig. 5.3). Previous reports that an active enzyme can be reconstituted by fragment complementation of the human Top1 core (expressed in SF9 

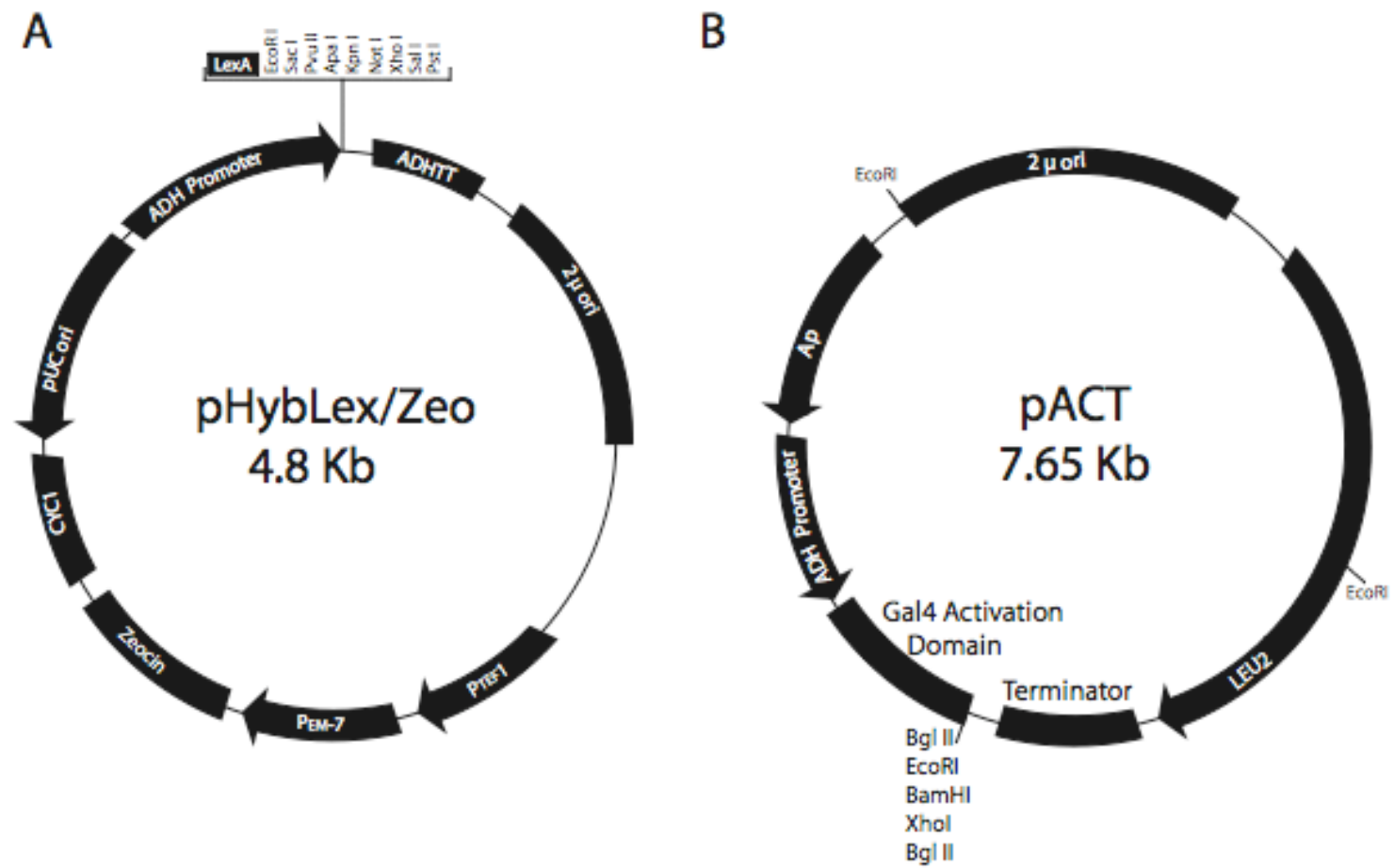

FIG. 5.1. Plasmids used for the construction of the bait and the prey. $A$. yTop $1^{\mathrm{Link} / \mathrm{C}-1}$ and yTop $1^{\text {Link/C-2 }}$ were amplified and ligated into the EcoRI and SacI sites of $\mathrm{pHybLex} /$ Zeo, while hTop $1^{\text {Link/C }}$ was ligated into the SacI and KpnI sites. B. S.cerevisiae cDNAs were ligated into the XhoI site located in the pACT sequences embedded in $\lambda$ DNA flanked by LoxP sites. Site specific recombination by the Cre recombinase generates a library of pACT plasmids that contain random yeast cDNA inserts, Nterminally HA tagged and located downstream of the Gal4 activation domain (AD). $\sim 1 / 3$ are expected to be in frame with the Gal4 AD and therefore encode a chimeric prey. 
A

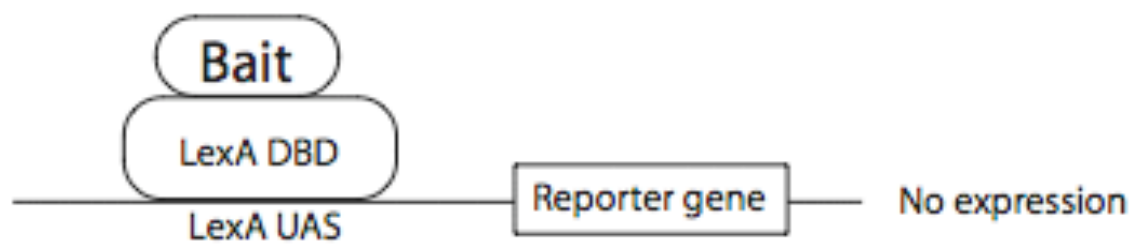

B
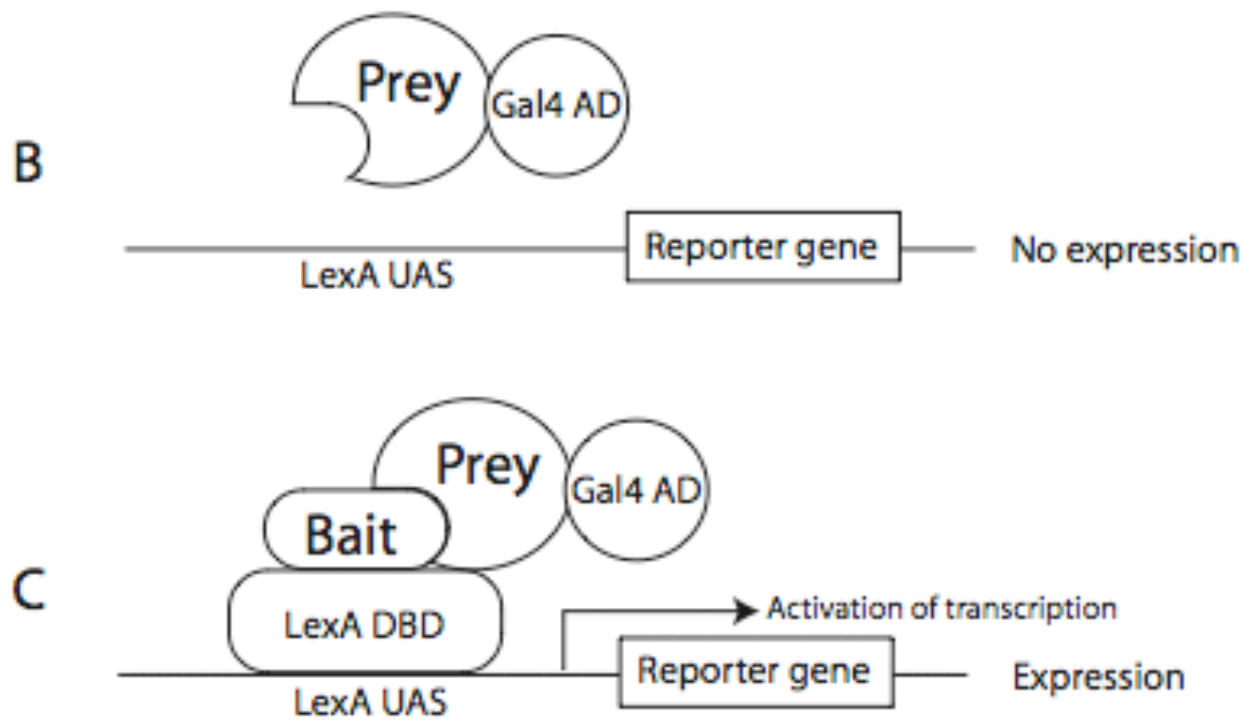

FIG. 5.2. Schematic representation of the two-hybrid system. $A$ and $B$. The LexA $\mathrm{DBD} /$ Bait or the Gal4 AD/Prey fusion proteins alone are unable to activate transcription. $C$. Interaction of the Bait and Prey will recruit the Gal4 AD to the LexA DBD and together they will form a functional unit that is able to activate transcription. 


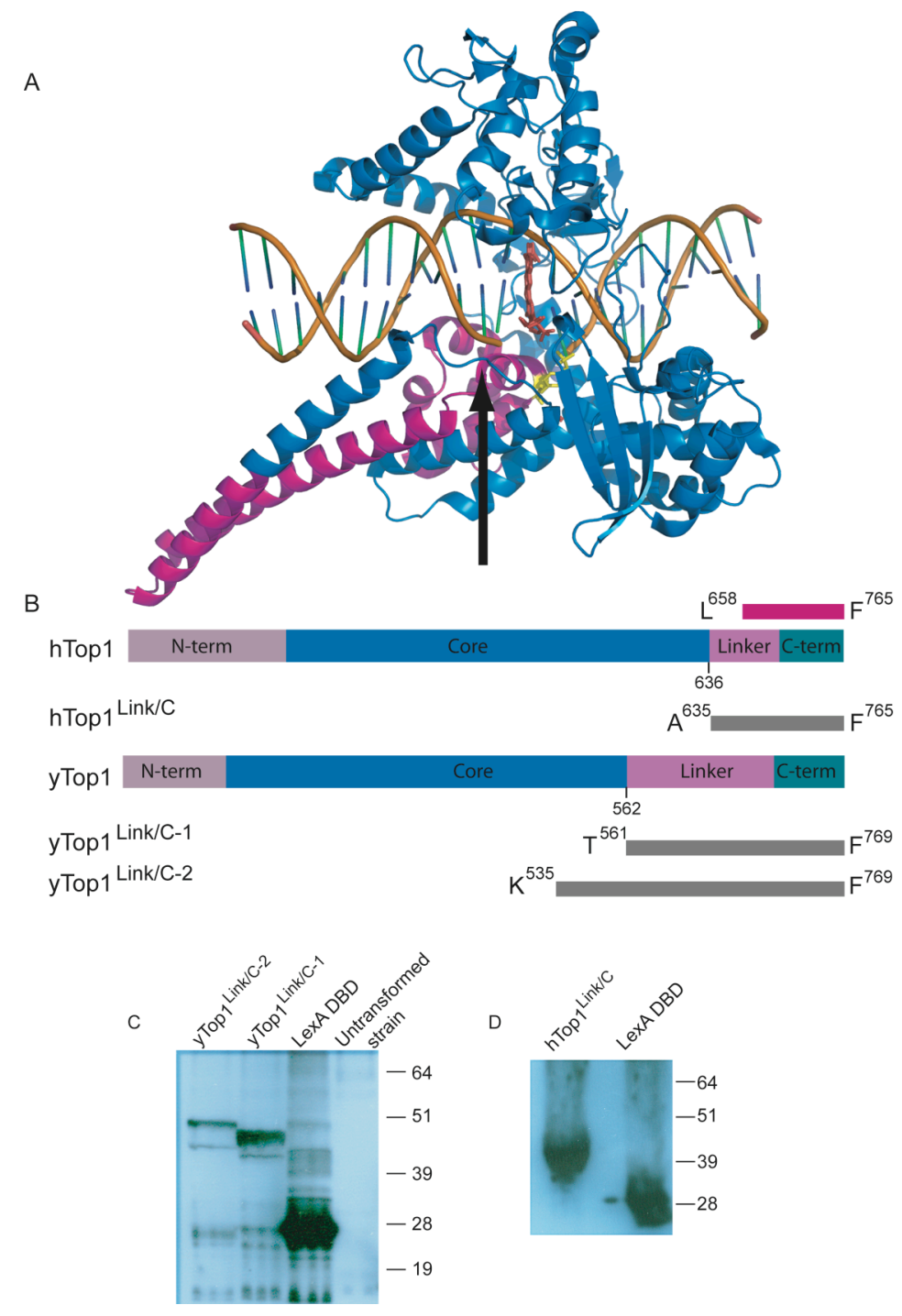

FIG. 5.3. Polypeptides used as bait. $A$. Structure of the C-terminal $70 \mathrm{kDa}$ human Top 1 (Topo70) covalently linked to duplex DNA in the presence of topotecan (TPT) [PDB file $1 \mathrm{~K} 4 \mathrm{~T}$ (59)]. TPT is indicated in red and the active site $\mathrm{Tyr}^{723}$ is in yellow. The region in magenta corresponds to the residues used by Stewart et al. (39) to reconstitute an active enzyme when combined with the hTop 1 core. The black arrow indicates the $\mathrm{Thr}^{561}$ which is the beginning of the bait that was used in the screen. $B$. Schematic representation of the domain organization of yeast and human Top1. The magenta bar at the top corresponds to the region (shown in magenta in $A$ ) that was used for reconstitution experiments and the gray bars represent the regions of human and yeast Top 1 that was used to generate the bait constructs. yTop $1^{\text {Link/C-1 }}$ was used for the two-hybrid screen. $C$ and $D$. Levels of the fusion proteins in crude cells extracts were determined in immunoblots with the LexA antibody. Expression of the LexA DBD by itself was used as a positive control. LexA DBD is $26 \mathrm{kD}$, yTop $1^{\mathrm{Link} / \mathrm{C}-1}$ is $49 \mathrm{kD}$, yTop $1^{\mathrm{Link} / \mathrm{C}-2}$ is $52 \mathrm{kD}$ and $\mathrm{hTop} 1^{\mathrm{Link} / \mathrm{C}}$ is $40 \mathrm{kD}$. 
insect cells) with the linker/C-terminal domain (expressed in E.coli and represented in magenta Fig. 5.3.A and B), suggest proper folding of both polypeptides. Based on these results and the sequence alignment of yeast and human Top1, two bait constructs were generated. yTop $1^{\mathrm{Link} / \mathrm{C}-1}\left(\mathrm{Thr}^{561}-\mathrm{Phe}^{769}\right)$ includes the complete linker plus C-terminal domain, while yTop $1^{\text {Link/C-2 }}\left(\mathrm{Lys}^{535}-\mathrm{Phe}^{769}\right)$ starts within the C-terminal portion of the core, corresponding to exon 17/18 junction of hTopl, and includes the linker and Cterminal domains ( polypeptides are indicated in gray in Fig. 5.3.B). Both constructs were examined for stable protein expression in immunoblots of yeast cell extracts (Fig. 5.3.C). As yTop $1^{\text {Link/C-1 }}$ fusion protein levels were consistently higher than that obtained with the yTop $1^{\mathrm{Link} / \mathrm{C}-2}$ bait, and this construct was restricted to only the linker and C-terminal domains, yTop $1^{\mathrm{Link} / \mathrm{C}-1}$ was used as bait in the screen (Fig 5.3.C). As the C-terminal domain is largely buried within the co-crystal structures of Topo70 and DNA, we further reasoned that any artifactual protein binding to C-terminal residues would be eliminated in subsequent studies with full length Top1 proteins. A construct containing the hTop $1^{\text {Link/C }}$, which corresponds to the yTop ${ }^{\text {Link/C-1 }}$, was also generated and tested for stable expression of the LexA DBD-hTop $1^{\text {Link/C }}$ fusion protein (Fig. 5.3.D).

To confirm that the LexA DBD-yTop $1^{\text {Link/C-1 }}$ fusion protein did not activate transcription of the reporter genes, $\mathrm{pHybLex} / \mathrm{Zeo}-\mathrm{yTop} 1^{\mathrm{Link} / \mathrm{C}-1}$ was co-transformed with the pACT (vector backbone of the cDNA library), which encode only the Gal4 AD polypeptide, into L40 yeast cells. The transforments were assayed for histidine prototrophy and $\beta$-galactosidase activity, the yTop $1^{\text {Link/C-1 }}$ fusion protein failed to induce HIS3 or lacZ gene transcription (Data not shown). 
For an unbiased screen of protein interactions with the linker and C-terminal domains of yeast Top1, the prey used comprised of a yeast cDNA library. It was cotransformed with $\mathrm{pHybLex} / \mathrm{Zeo}-\mathrm{yTop} 1^{\mathrm{Link} / \mathrm{C}-1}$ and transformants selected on histidinedeficient plates. Positive interactions would allow growth on $\mathrm{His}^{-}$plates. $\mathrm{His}^{+}$colonies were a rare event relative to the number of primary transformants (plating $100 \mu 1$ of a $1 \times 10^{-6}$ dilution of the primary transformants on $\mathrm{His}^{+}$plates gave a lawn of cells, while 5 $\mu l$ of the transformation reaction plated on His ${ }^{-}$plates generated about 8 $\mathrm{His}^{+}$colonies/plate) and only $50 \%-60 \%$ of the $\mathrm{His}^{+}$colonies expressed active $\beta$ galactosidase. Plasmids were isolated from $70 \mathrm{His}^{+}, \beta$-galactosidase ${ }^{+}$colonies and 16 potential interactors identified (listed in Table 5.1).

Many of the cDNAs retrieved from the screen were not full length. For example, all of the POL30 (PCNA) clones sequenced are missing the N-terminal 172 bases. Complete coding regions were identified for UBC9, NSE5 and MRM1.

Ubc9, Nis1 and Pol30, but not Mrm1 interact with both yeast and human linker and C-terminal domain. Based on the number of clones identified for each cDNA and interactions with Top1 reported in the literature, the following potential interactors were chosen for the subsequent experiments; Ubc9, Nis1, Pol30, Mrm1 and Nse5 (all fused to the Gal4 AD). We first confirmed the interaction between these proteins and yTop $1^{\text {Link/C-1 }}$ and then assessed potential interactions with hTop $1^{\text {Link/C }}$ (Table 5.2). Each fusion protein was coexpressed with yTop $1^{\text {Link/C-1 }}$, hTop $1^{\text {Link/C }}$ (both fused to a LexA DBD) or a polypeptide corresponding to the LexA DNA binding domain. The proteins were expressed in the L40 yeast strain that contains the HIS3 reporter gene and the subsequent transformants were grown on His ${ }^{-}$plates in the presence or absence of $3 \mathrm{mM} 3-$ 
TABLE 5.1. Proteins identified in the two-hybrid screen.

\begin{tabular}{|c|c|c|}
\hline Name & \# & Function (Saccharomyces genome database) \\
\hline${ }^{\mathrm{a}}$ Tif4631 & 1 & $\begin{array}{l}\text { Translation initiation factor eIF4G, subunit of the mRNA cap-binding } \\
\text { protein complex (eIF4F) that also contains eIF4E (Cdc33p); associates } \\
\text { with the poly(A)-binding protein Pab1p, also interacts with eIF4A } \\
\text { (Tif1); homologous to Tif4632 }\end{array}$ \\
\hline${ }^{\mathrm{a}} \mathrm{Rsc} 9$ & 1 & $\begin{array}{l}\text { Component of the RSC chromatin remodeling complex; DNA-binding } \\
\text { protein involved in the synthesis of rRNA and in transcriptional } \\
\text { repression and activation of genes regulated by the Target of } \\
\text { Rapamycin (TOR) pathway }\end{array}$ \\
\hline${ }^{\mathrm{a}} \mathrm{Asc} 1$ & 3 & $\begin{array}{l}\text { WD repeat protein (G-beta like protein) involved in translation } \\
\text { regulation; required for repression of Gcn } 4 \text { activity in the absence of } \\
\text { amino-acid starvation; core component of the ribosome; ortholog of } \\
\text { mammalian RACK1 }\end{array}$ \\
\hline${ }^{\mathrm{b}} \mathrm{Ubc} 9$ & 2 & $\begin{array}{l}\text { SUMO-conjugating enzyme involved in the Smt } 3 \text { conjugation } \\
\text { pathway; nuclear protein required for S- and M-phase cyclin } \\
\text { degradation and mitotic control; involved in proteolysis mediated by } \\
\text { the anaphase-promoting complex cyclosome }\end{array}$ \\
\hline${ }^{\mathrm{b}} \mathrm{Siz} 2$ & 1 & $\begin{array}{l}\text { SUMO ligase, catalyzes the covalent attachment of SUMO }(\mathrm{Smt}) \text { to } \\
\text { proteins; involved in maintenance of proper telomere length }\end{array}$ \\
\hline${ }^{\mathrm{b}} \mathrm{Nse} 5$ & 4 & $\begin{array}{l}\text { Essential subunit of the Mms21-Smc5-Smc6 complex; required for cell } \\
\text { viability and DNA repair }\end{array}$ \\
\hline${ }^{\mathrm{c}} \mathrm{His} 7$ & 1 & $\begin{array}{l}\text { Imidazole glycerol phosphate synthase (glutamine } \\
\text { amidotransferase:cyclase), catalyzes the fifth and sixth steps of } \\
\text { histidine biosynthesis and also produces } 5 \text {-aminoimidazole-4- } \\
\text { carboxamide ribotide (AICAR), a purine precursor }\end{array}$ \\
\hline${ }^{\mathrm{c}} \mathrm{Pdc} 1$ & 1 & $\begin{array}{l}\text { Major of three pyruvate decarboxylase isozymes, key enzyme in } \\
\text { alcoholic fermentation, involved in amino acid catabolism }\end{array}$ \\
\hline${ }^{\mathrm{c}}$ Prs2 & 1 & $\begin{array}{l}\text { 5-phospho-ribosyl-1(alpha)-pyrophosphate synthetase, involved in } \\
\text { nucleotide, histidine, and tryptophan biosynthesis; one of a five related } \\
\text { enzymes, which are active as heteromultimeric complexes }\end{array}$ \\
\hline${ }^{\mathrm{d}} \mathrm{Rps} 19 \mathrm{~A}$ & 8 & $\begin{array}{l}\text { Protein component of the small ( } 40 \mathrm{~S}) \text { ribosomal subunit, required for } \\
\text { assembly and maturation of pre- } 40 \mathrm{~S} \text { particles }\end{array}$ \\
\hline
\end{tabular}


TABLE 5.1. (continued)

\begin{tabular}{|c|c|c|}
\hline Name & \# & Function (Saccharomyces genome database) \\
\hline${ }^{\mathrm{d}} \mathrm{Rpl3}$ & 1 & Protein component of the large (60S) ribosomal subunit \\
\hline${ }^{\mathrm{e}} \mathrm{Nis} 1$ & 17 & $\begin{array}{l}\text { Protein localized in the bud neck at G2/M phase; physically interacts } \\
\text { with septins; possibly involved in a mitotic signaling }\end{array}$ \\
\hline${ }^{\mathrm{e}} \mathrm{Mrm} 1$ & 7 & $\begin{array}{l}\text { Ribose methyltransferase that modifies a functionally critical, } \\
\text { conserved nucleotide in mitochondrial } 21 \mathrm{~S} \text { rRNA }\end{array}$ \\
\hline${ }^{\mathrm{e}} \mathrm{Clf} 1$ & 1 & $\begin{array}{l}\text { Essential splicesome assembly factor; contains multiple } \\
\text { tetratricopeptide repeat (TPR) protein-binding, may serve as a scaffold } \\
\text { during splicesome assembly }\end{array}$ \\
\hline${ }^{\mathrm{e}} \mathrm{Swd} 3$ & 1 & $\begin{array}{l}\text { Essential subunit of the COMPASS (Set1C) complex, which } \\
\text { methylates histone } \mathrm{H} 3 \text { on lysine } 4 \text { and is required in transcriptional } \\
\text { silencing near telomeres }\end{array}$ \\
\hline $\begin{array}{l}{ }^{\mathrm{e}} \mathrm{Pol} 30 \\
\text { (PCNA) }\end{array}$ & 4 & $\begin{array}{l}\text { Proliferating cell nuclear antigen (PCNA), functions as the sliding } \\
\text { clamp/processivity factor for DNA polymerase delta; may function as a } \\
\text { docking site for other proteins required for mitotic and meiotic } \\
\text { chromosomal DNA replication and for DNA repair }\end{array}$ \\
\hline
\end{tabular}

${ }^{\text {a }}$ Proteins involved in TOR (Target of Rapamycin) signaling. Tif4631 has been shown to physically interact with Top1p through affinitycapture-ms (191).

${ }^{b}$ Proteins involved in sumoylation. Ubc9 genetically interacts with Top1 (136).

${ }^{\mathrm{c}}$ Proteins involved in amino acid metabolism, more specifically histidine biosynthes. As these proteins are involved in histidine metabolism, they might restore histidine prototrophy and potentially be false positives.

${ }^{\mathrm{d}}$ Proteins that form components of the ribosome. Rps19A is a ribosomal protein that is highly expressed and therefore might lead to non-functional interactions.

${ }^{\mathrm{e}}$ Miscellaneous proteins. Nis1 is located in the bud neck during $\mathrm{G}_{2} / \mathrm{M}$ phase, while Top1 is a nuclear protein and therefore this might not be a functional interaction. Clf1, which is involved in spliceosome assembly, is an interesting find as the Top $1 \mathrm{~N}$-terminal domain is known to bind to other spliceosomal proteins SF2/ASF (137). Mammalian PCNA has been shown to interact with human Top1 by affinity chromatography (192).

\# refer to the number of clones identified in screen. 
Aminotriazol (3-AT). A positive interaction is indicated by growth on plates lacking histidine. Strong and weak interactions can be discriminated based on growth on plates containing 3-Aminotriazole. Fig. 5.4. shows an example of the above mentioned experiment using Ubc9, while Table 5.2. provides a summary of results for all the interactors tested (Ubc9, Pol30, Mrm1, Nis1and Nse5).

Expression of the Nse5 fusion protein with the LexA DBD by itself cause growth on His- plates, indicating that this interaction is not dependent on Top1 and is therefore scored as a false positive. Ubc9, Pol30 and Nis1 interact with both the yTop1 ${ }^{\mathrm{Link} / \mathrm{C}-1}$ and hTop $1^{\text {Link/C }}$, albeit to a lesser extend with the human protein. Mrm1 interacts strongly with the yTop1 ${ }^{\text {Link/C-1 }}$, but no interaction was detected with hTop1 $1^{\text {Link/C }}$.

Next, immunoprecipitations were performed to confirm the physical interactions defined with the two-hybrid screen. As the cDNAs were ligated into the $\lambda$ vector that contains sequence encoding a HA-tag N-terminal to the inserted cDNA, the Gal4 ADfusion protein can be detected in immunoblots using the HA-antibody. Fusion proteins of the predicted size were detected for Nis1, Nse5, Pol30 (PCNA), Mrm1 and Rps19A (Data not shown). Cells expressing these fusion proteins also contain a protein product that is $12 \mathrm{kD}$ smaller than the fusion protein and corresponds to the protein without the Gal4 AD. Even though a correct size band was detected for the Ubc9 fusion protein (29kD), a second band $(\sim 43 \mathrm{kD})$ was also observed. It had been reported that Ubc9 are able to form dimers through a thioester bond (193), but as the protein was resolved in the presence of the reducing agent, $0.1 \mathrm{M}$ dithiothreitol (dtt), it is unlikely that the extra band is a dimer. Ubc9 has also been shown to be sumoylated on Lys ${ }^{153}$, which is a non-consensus SUMO- 


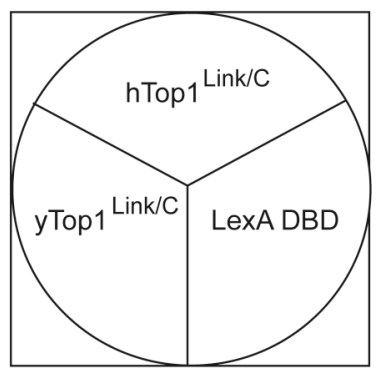

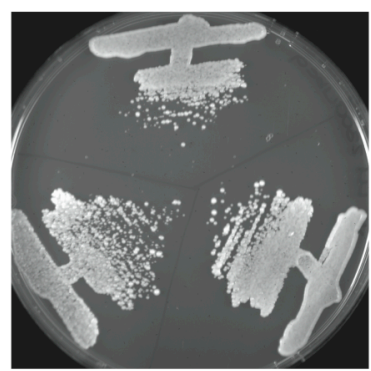

$\mathrm{His}^{+}$

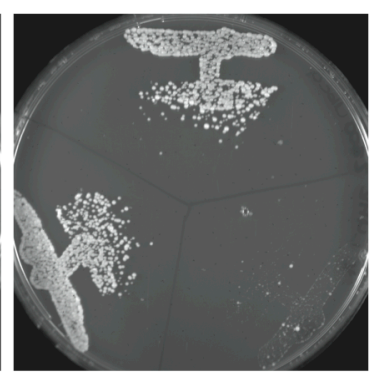

$\mathrm{His}^{-}$

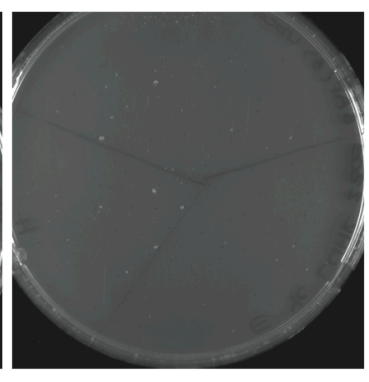

$\mathrm{His}^{-}, 3-\mathrm{AT}$

FIG. 5.4. Ubc9 interacts with yTop $1^{\mathrm{Link} / \mathrm{C}-1}$ and hTop $1^{\mathrm{Link} / \mathrm{C}}$ equally well. Gal4 ADUbc9 fusion protein coexpressed with LexA DBD-hTop $1^{\text {Link/C }}$ fusion protein, LexA DBD-yTop $1^{\text {Link/C-1 }}$ fusion protein or the LexA DBD by itself, were assayed for growth on plates deficient for histidine in the presence or absence of 3-AT. 
TABLE 5.2. Distinct interactions with yTop $1^{\mathrm{Link} / \mathrm{C}-1}$ and $h T o p 1^{\mathrm{Link} / \mathrm{C}}$.

\begin{tabular}{lccccc}
\hline & Ubc9 & Pol30 & Mrm1 & Nis1 & Nse5 \\
\hline Yeast Bait & + & ++ & ++ & ++ & + \\
Human Bait & + & + & - & + & + \\
LexA DBD & - & - & - & - & + \\
\hline
\end{tabular}

$(-)$ indicates no growth on a His ${ }^{-}$plate, $(+)$indicates growth on a His ${ }^{-}$plate and $(++)$ indicates growth on His plates containing $3 \mathrm{mM}$ 3-AT. 
site (194). The size of the higher molecular band corresponds to a polypeptide that is potentially a sumoylated Ubc9-fusion protein.

For immunoprecipitations, Ubc9, Mrm1, Pol30, Nse5, Nis1 or Rps19A

fusionproteins were coexpressed with yTop $1^{\text {Link/C-1 }}$-LexA fusion protein (Fig.5.5).

Immunoprecipitation was performed using the LexA antibody, followed by

immunoblotting with HA-antibody. A physical interaction with the yeast linker and Cterminal domain could only be detected for Mrm1 and Pol30 (PCNA).

\subsection{DISCUSSION}

Top1 interacts with various protein partners such as TATA binding protein and nucleolin $(34,100)$. All of these interactions are dictated by the N-terminal domain of Top1. From studies using a chimeric Top1 protein, where the human Top1 linker domain has been replaced by the much larger yeast linker, it was found that this protein was toxic to yeast cells when overexpressed from GAL1 promoter. In vitro experiments, using purified protein showed no difference between hTop1 and this chimeric protein in specific activity or covalent complex formation in the presence and absence of CPT. Moreover, there was also no difference in the optimum salt concentration for specific acitivity between these two proteins, indicating that it is not a binding defect that is inducing the toxicity. Further experiments showed that the catalytically inactive chimeric protein is also toxic, suggesting that the toxicity is not due to the catalytic activity (Chapter 4). This led us to hypothesize that the linker domain might be involved in protein-protein interactions that differ between the yeast and human Top1 proteins. 


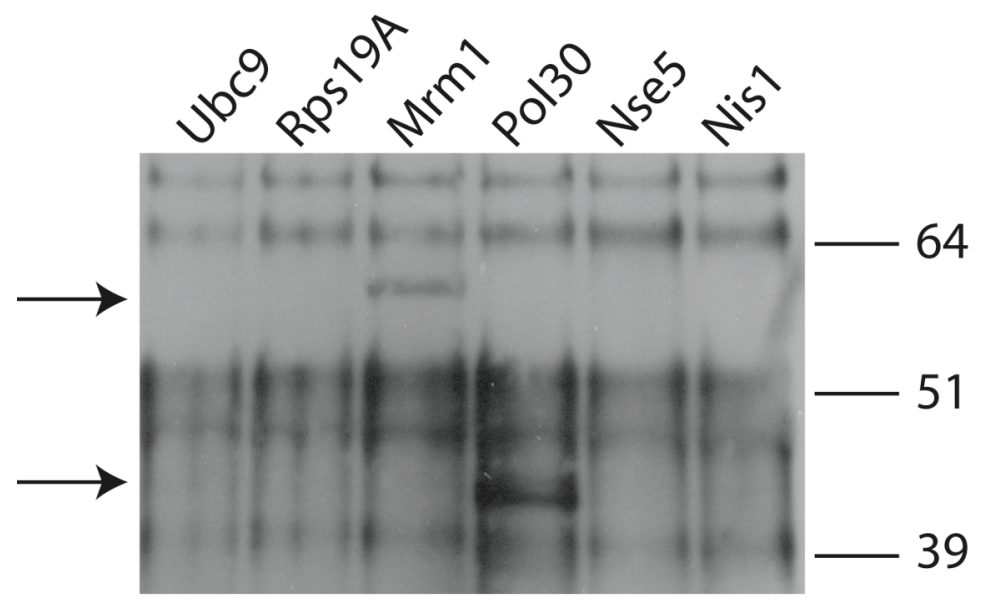

FIG 5.5. Mrm1 and Pol30 physically interact with yTop $1^{\mathrm{Link} / \mathrm{C}-1}$. YTop ${ }^{\mathrm{Link} / \mathrm{C}-1}$ fused to the LexA DBD was coexpressed with Gal4 fused HA-tagged Ubc9, Rps19A, Mrm1, Pol30, Nse5 and Nis1. Immunoprecipitation was performed using the LexA antibody and immunoblotting with an HA antibody. 
Using a two-hybrid system where the bait was the yeast Top1 linker and Cterminal domain (yTop $1^{\text {Link/C-1 }}$ ), we screened a S.cerevisiae cDNA library for proteins that interact with this region of Top1. From the screen we identified 16 proteins that allowed for growth on selective media and also activated transcription of the reporter gene, lacZ. Of the 16 proteins, three are involved in amino acid metabolism, Prs2, His7 and Pdc1 are potentially false positive.

Nis1, Mrm1, Pol30 and Ubc9 fusion proteins were confirmed as true interactors with $y \mathrm{Top}^{\mathrm{Link} / \mathrm{C}-1}$ domain and tested for interaction with human Top1 linker and Cterminal domain. Pol30 and Nis1 interact with both the human and yeast linker and Cterminal domain, with a slight decrease in binding to the human protein. A physical interaction between $y$ Top $1^{\text {Link/C-1 }}$ and Pol30 was further confirmed by immunoprecipitaion. An interaction between full length yTop1 and Pol30 was further demonstrated by a postdoctoral fellow in the lab, Clifford Toleman, also using immunoprecipitations. As a Top1 interactor, Pol30 (PCNA) is of specific interest as it acts as a sliding clamp moving along with the replication fork and functions as a scaffold with which various replication proteins interact (195). During replication Top1 acts as a swivel to relieve torsional strain created by the movement of the replication fork. It is therefore plausible that crosstalk exists between Top 1 and Pol30. The function of this interaction can be to either recruit Top1 to DNA regions that is under increased torsional strain (due to ongoing replication) or a mechanism for Top1 to sense the approaching replication fork.

Ubc9 interacts with the yeast and human protein equally well. Even though the interaction with yTop1 ${ }^{\text {Link/C-1 }}$ with Ubc9 could not be confirmed with the 
immunoprecipitation, it is still an intriguing result. Ubc9 is the second enzyme (E2) in the sumoylation cascade and can either by itself or with the help of an E3 ligase transfer SUMO (Smt3 in yeast) to a Lysine on the target protein. The regions of both yTop $1^{\text {Link/C-1 }}$ and hTop $1 p^{\text {Link/C }}$ used in the bait constructs contained consensus SUMO-sites, one in yTop $1 p^{\text {Link/C-1 }}\left(\operatorname{Lys}^{600}\right)$ and two in hTop $1^{\text {Link/C }}\left(\operatorname{Lys}^{654}\right.$, Lys $\left.^{669}\right)$, and it is therefore possible that Ubc9 is binding to these regions. There had been conflicting reports in the literature about the role of sumoylation of Top1. It was initially shown that sumoylation of hTop1 in response to high concentration of the anticancer drug, camptothcin (CPT), resulted in relocalization of the enzyme from the nucleus to the nucleolus (134). However, more recent data using life cell imaging concluded that this modification did not affect CPTinduced nucleolar transport (122). Moreover, mutations of the sumoylation sites on Top1 had no effect on CPT sensitivity, indicating the sumoylation of Top1 does not affect CPT sensitivity directly (136). In the immunoblot results of Ubc9, there were two protein populations, a band corresponding to the Gal4-Ubc9 fusion protein and then a second band that is potentially a sumoylated Ubc9 fusion protein. Ubc9 has been shown to be sumoylated on Lys ${ }^{153}$, which is a non-consensus SUMO-site (194). This leads to the possibility that Top1 only interacts with either sumoylated or unsumoylated Ubc9. This issue can be resolved by mutating Lys ${ }^{153}$ on Ubc9 and testing if the interaction between Ubc9p and yTop $1^{\mathrm{Link} / \mathrm{C}-1}$ still exists.

Another protein identified in this screen that is involved in the sumoylation pathway is Siz2. This protein is a SUMO E3 ligase that increases the efficiency of SUMO conjugation. It functions by binding to Ubc9, and accelerate the rate of SUMO modification in a substrate specific fashion. It can therefore be imagined that the 
recruitment of Siz2 is not directly through the interaction with yTop $1^{\mathrm{Link} / \mathrm{C}-1}$, but rather indirectly through the binding of Ubc9. As Ubc9 is an essential protein and the binding region for E3's overlap with the binding region of E1's, mutating Ubc9 to loose the interaction with Siz2 will be problematic. A more feasible approach would be to generate recombinant proteins and determine interaction using electromobility shift assays. Using this technique, it can be established if Siz2 binds to Ubc9p or yTop $1^{\mathrm{Link} / \mathrm{C}-1}$ individually, or if it requires both proteins for binding.

From the proteins that had been tested for differential binding between the yTop $1^{\text {Link/C-1 }}$ and hTop $1^{\text {Link/C }}$, Mrm1 was the only protein that interacted strongly with the yeast bait and not at all with the human bait. It also physically interacted with yTop $1^{\mathrm{Link} / \mathrm{C}-1}$ in immunoprecipitations. Mrm1 is a ribose methyltransferase that is essential for the formation functional mitochondrial ribosomes. No connections have been made between this protein and any Top1 function.

This study was the first step in identifying proteins that interact with the Top1 linker domain. We found various binding partners that had already been shown to have physical or genetic interaction with Top1, such as Pol30 (PCNA) and Ubc9. The next challenge would be to determine what the biological meaning of these interactions are in the context of regulating Top1 function and Top1 targeted drug sensitivity. 


\section{CHAPTER 6: DISCUSSION}

DNA topoisomerase IB (Top1) is a highly conserved enzyme found in all phyla of the evolutionary tree and is capable of relaxing both positive and negative supercoils in the absense of an energy donating cofactor $(4,7)$. Top1 became medically important with the discovery that it is the sole target of the anti-tumor drug camptothecin, CPT, a cytotoxic plant alkaloid $(6,57,67)$. CPT and its derivatives act by intercalating between the ends of the cleaved strand, interacting with both the DNA and the enzyme, preventing the religation step of the catalytic cycle and extending the lifetime of the covalently linked Top1-DNA intermediate $(59,196)$. This mechanism of action is unique as it not the inhibition of catalytic activity that is toxic to the cell, but the replication machinery moving along the DNA and encountering the covalently linked intermediate that results in irreversible lesions which leads to cell death (181). Dogma has held that it is the actual collision between the replication machinery and drug stabilized enzyme that creates the cytotoxic lesions. However, more recent in vitro and in vivo data demonstrate that in the presence of CPT there is a decrease in the relaxation of positive supercoils, suggesting that in the context of an advancing replication fork it might be the accumulation of positive supercoils between the covalent Top1 lesion and the advancing replication fork, rather that the actual collision, that is inducing the DNA lesions (104). Because of this mechanism of cytotoxicity, understanding the downstream pathways that are affected by the formation of this DNA lesion, is of critical importance. 


\subsection{MUTATIONS IN THE ACTIVE SITE REGION OF TOP1 INDUCE DISTINCT SELF POISONING ACTIVITIES}

yTop $1 \mathrm{~N}^{726} \mathrm{H}$ has an increased rate of cleavage, while yTop $1 \mathrm{~T}^{722} \mathrm{~A}$ has a decrease in the rate of religation, mimicking the action of CPT (103). Even though the result of both mutants is an increase in covalent complex formation, yeast cell sensitivity to constitutively low level expression of these mutants in different genetic backgrounds varied dramatically. A model whereby these mutants do not induce different levels of DNA damage, but actually create distinct lesions is supported by the variable patterns of cytotoxicity observed when these mutants were expressed in a panel of isogenic tah mutant strains. The tah strains were originally isolated in a genetic screen for enhanced sensitivity to low levels of yTop $1 \mathrm{~T}^{722} \mathrm{~A}$ at a non-perminssive temperature of $36{ }^{\circ} \mathrm{C}(120)$. As shown in chapter 2 , the $c d c 45-10$ strain can tolerate low levels of yTop $1 \mathrm{~T}^{722} \mathrm{~A}$ at 26 ${ }^{\circ} \mathrm{C}$ (permissive temperature), but had decreased transformation efficiency with the same vector expressing yTop $1 \mathrm{~N}^{726} \mathrm{H}$. These cells contain a temperature sensitive hypomorphic allele of $C D C 45$ and are defective in processive DNA replication at the non-permissive temperature. A second strain, defective in processive DNA replication at the nonpermissive temperature, $d p b 11-10$, could not tolerate the yTop $1 \mathrm{~N}^{726} \mathrm{H}$ at any temperature. This result indicates that Cdc45 and Dpb11 are involved in either the sensing or repair of the damage induced by these specific mutants. In contrast, doa4-10 cells, which contains a nonsense mutation in $D O A 4$, are resistant to expression of yTop $1 \mathrm{~N}^{726} \mathrm{H}$ and yTop $1 \mathrm{~N}^{726} \mathrm{~F}$ at the non-permissive temperature. Doa4 is an ubiquitin C-terminal hydrolase, which functions to maintain free ubiquitin pools. The generation such variable phenotypes in the different genetic backgrounds indicate that the mechanism of lesion 
formation affects the cells' response to the lesions induced. It can be imagined that in the intracellular multi-protein environment, different protein complexes will sense and repair distinct lesions and that understanding these downstream sensing and repair pathways will be important in the development of therapeutics targeting Top1.

With the knowledge that distinct Top1-induced lesions exist, it will be of great interest to perform additional genetic screens to define distinct and common downstream pathways that regulate cellular responses to DNA lesions induced by Top $1 \mathrm{~N}^{726} \mathrm{H}$ and

Top $1 \mathrm{~T}^{722} \mathrm{~A}$. As a conditional yTop $1 \mathrm{~T}^{722} \mathrm{~A}$ sensitive screen has already been performed, a second screen using yTop $1 \mathrm{~N}^{726} \mathrm{H}$ can be done to compare conditional mutant proteins between these two screens. Another great resource for the yeast research community is the existence of yeast gene deletion libraries, which contains a collection of strains where all the non-essential genes are deleted. With the development of robotic platforms, the use of this deletion set is now feasible and one should be able to use this technology to define genes that suppress cell sensitivity to, for example, the yTop $1 \mathrm{~N}^{726} \mathrm{H}$ mutant.

\subsection{THE GLY RESIDUE LOCATED IN THE TRANSITION REGION BETWEEN THE LINKER AND THE ACTIVE SITE TYR ACTS AS A HINGE TO ALTER ACTIVE SITE GEOMETRY AND LINKER FLEXIBILITY AND THEREBY INFLUENCE CPT SENSITIVITY}

Crystallographic data revealed Top1 as a protein clamp that wraps around duplex DNA, with the linker domain forming a coiled coil structure extending away from the body of the enzyme $(12,59)$. The linker also positions the $\mathrm{C}$-terminal active site domain 
within the catalytic pocket formed by the core. Several lines of evidence suggest that the linker domain is a critical determinant of CPT sensitivity. For instance, altering the flexibility of this domain either by physically disconnecting the linker from the core (as in the reconstituted Topo70 preparations) or through a mutation $\left(\mathrm{A}^{653} \mathrm{P}\right)$ in the linker domain of hTop1, CPT sensitivity was decreased $(61,62)$. Crystallographic data of the covalent Topo70-DNA complex demonstrated that in the presence of the CPT analog, topotecan, there is a decrease in linker domain flexibility enabling the structural determination of the coiled coil. This structure is not visible in the binary Top1-DNA covalent complex, indicating increased linker domain movement (59). A closer assessment of the region of the enzyme that connects the coiled coil to the active site Tyr domain revealed a subtle change in structure. A $\alpha$-helix extending from the active site Tyr towards the core is extended in the presence of the drug to include the conserved residues Leu ${ }^{716}$ and $\mathrm{Gly}^{717}\left(\mathrm{Leu}^{720}, \mathrm{Gly}^{721}\right.$ in yeast Top1). These data suggest the existence of a dynamic relationship between intercalation of the drug, alterations in active site $\alpha$-helical structure and the flexibility of the linker domain. Such long range molecular interactions between the linker and active site in mediating enzyme sensitivity to CPT are further supported by recent studies in which the cytotoxicity induced by a defect in human Top1-catalyzed DNA religation (due to the active site mutation $\mathrm{T}^{722} \mathrm{~A}$ ) was suppressed by the increased linker flexibility of the $\mathrm{A}^{653} \mathrm{P}$ mutation (197).

Based on these considerations, we hypothesized that the Gly located in the transition region between the linker and the active site Tyr acts as a flexible hinge to alter active site geometry and linker flexibility and thereby influence CPT sensitivy. In vitro and in vivo analyses of $\mathrm{Gly}^{721}$ mutations in yeast Top1p suggest that the charge and $\alpha$ - 
helical propensity of amino acid side chains at position 721 directly impact active site architecture within the covalent enzyme-DNA complex and the intrinsic sensitivity of Top1 to CPT.

Our mutational analyses of substitutions at position 721 demonstrate that the introduction of a bulky aromatic residue (Phe) or $\beta$ branched aliphatic side chain (Val) decreases the specific activity and concomitantly the CPT sensitivity of Top1. In contrast, the introduction of an acidic side chain, with carboxyl groups at the $\beta$ or $\gamma$ carbon in $\mathrm{G}^{721} \mathrm{D}$ and $\mathrm{G}^{721} \mathrm{E}$, respectively, dramatically enhanced Top1p sensitivity to CPT, without any obvious alterations in specific enzyme activity in plasmid DNA relaxation assays or increase in covalent complex formation in steady state DNA cleavage assays. The negative charge of Asp and Glu appears to enhance drug sensitivity as the increased CPT sensitivity of cells expressing Top1 mutants engineered with the amide forms of these residues $\left(\mathrm{G}^{721} \mathrm{~N}\right.$ and $\left.\mathrm{G}^{721} \mathrm{Q}\right)$ and the $\gamma$ branched aliphatic side chain $\left(\mathrm{G}^{721} \mathrm{~L}\right)$ was only evident in the absence of the DNA damage checkpoint. In addition to charge, a second determinant of CPT sensitivity appears to be the tendency of residues at this position to form $\alpha$-helices, as mutating Gly ${ }^{721}$ to Ala [estimated to have the highest propensity for helix formation(178)] also increased cell sensitivity to CPT. These data indicate the following rank order of mutant-induced increased in Toplp sensitivity to CPT: $\left(\mathrm{G}^{721} \mathrm{E}\right.$, $\left.\mathrm{G}^{721} \mathrm{D}\right)>\mathrm{G}^{721} \mathrm{~A}>\left(\mathrm{G}^{721} \mathrm{~N}, \mathrm{G}^{721} \mathrm{Q}, \mathrm{G}^{721} \mathrm{~L}\right)>$ wild-type $>\mathrm{G}^{721} \mathrm{~V}>\mathrm{G}^{721} \mathrm{~F}$.

Our studies using purified protein and suicide substrates demonstrate that an increase in CPT sensitivity coincided with mutation induced alterations in enzyme active site structure. The $\mathrm{G}^{721} \mathrm{D}, \mathrm{G}^{721} \mathrm{E}$ mutants exhibited the greatest CPT sensitivity in vivo and in vitro, as well as specific alterations in tryptic digests of covalent enzyme-DNA 
complexes formed in DNA religation assays. Comparing the pattern of DNA-bound fragments obtained with the yeast and human Top1 mutants and correlating them with the potential trypsin cleavage sites in yeast and human Top1 amino acid sequence allowed us to determine the alterations in tryptic cleavage sites. In the residues spanning the active site tyrosine of yeast Top1p, $\underline{\underline{K}}^{712}$ EENSQYSLG ${ }^{721} \mathrm{TS}^{\underline{K}^{724}} \mathrm{INY}^{727} \mathrm{IDP} \underline{\underline{R}}$, trypsin digestion of SDS-denatured wild-type Top1p-DNA covalent complexes results in a 7 residue peptide (INY ${ }^{727}$ IDPR) covalently attached to a 5 ' end labeled 14 mer oligonucleotide via a 3'phosphotyrosyl linkage. Mutation of $\mathrm{Gly}^{721}$ to Asp or $\mathrm{Glu}\left(\mathrm{G}^{721} \mathrm{D}\right.$ or $\mathrm{G}^{721} \mathrm{E}$, respectively) decrease efficiency of trypsin digestion at $\mathrm{K}^{724}$ to yield longer peptides linked to the DNA. These longer peptides were not present when the protein was denatured with urea, indicating that the restricted tryptic digests are due to structural alterations in the mutant protein. One possibility is that the structure is altered such that trypsin cannot gain access to $\mathrm{K}^{724}$ and digestion is therefore restricted at this site. A second possibility is that the negative charge of $\mathrm{D}^{721}$ or $\mathrm{E}^{721}$ is precluding digestion. Position 721 is 3 amino acids away from the trypsin cleavage site at $\mathrm{K}^{724}$, therefore a structural alteration would still be required to position the negative charge in close enough proximity to $\mathrm{K}^{724}$ to impact trypsin digestion. As there are 3.6 amino acids per $\alpha$ helical turn, amino acids 3 or 4 residues apart are spatially quite close to each other. Our results therefore suggest that in the absence of CPT the mutant proteins have an extended $\alpha$-helical structure between Lys ${ }^{724}$ and position 721 , such that $\mathrm{D}^{721}$ or $\mathrm{E}^{721}$ are now in close proximity to $\mathrm{K}^{724}$. This proposed structural change would mimic what is seen in the co-crystal structure of the covalent Top1-DNA complex in the presence of topotecan, raising the possibility that these substitutions induce a structural change to create a pocket 
that facilitate CPT binding. To determine if it is the negative charge of $\mathrm{D}^{721}$ or $\mathrm{E}^{721}$ that precludes trypsin digestion at $\mathrm{K}^{724}$, trypsin digests of the CPT hypersensitivity $\mathrm{G}^{721} \mathrm{~A}$ mutant could be assessed and should reveal if it is charge by itself or the structural alteration that it leading to the diminished digestion at $\mathrm{K}^{724}$.

Using suicide substrates to uncouple cleavage from religation, we further demonstrated that the presence of duplex DNA 3' to the site of DNA cleavage site also altered the rates of Top $1 \mathrm{G}^{721} \mathrm{D}$ catalyzed DNA cleavage, religation, and the pattern of tryptic digests. This suggests that downstream protein-DNA contacts impact dynamic interactions between the active site Tyr/C-terminal domain and the linker. The human Top1 linker has been shown to be 10-fold more resistant to limited proteolysis when the enzyme is noncovalently bound to duplex DNA, consistent with linker-DNA contacts 3' to the site of DNA scission (28). An interesting observation from these experiments is that only when duplex DNA is present downstream of the cleavage site do we see the decrease in the rate of religation. As this assay is performed with an oligonucleotide that creates a flap at the site of cleavage, this extra dinucleotide might alter the active site pocket of $y \mathrm{y}_{\mathrm{T}} 1 \mathrm{pG}^{721} \mathrm{D}$, leading to a decrease in the rate of religation. This result is specific for the $\mathrm{G}^{721} \mathrm{D}$ mutant, but as there is no increase in the covalent complex formation in the absence of CPT in the steady state cleavage assays, this result might be an artifact of this assay or may reflect the distortion in the DNA structure induced by CPT binding.

The differences seen in tryptic digests in the presence and absence of downstream DNA, implies that there is an alteration in structure that allows cleavage at previously limited sites. This indicates that the presence or absence of duplex DNA downstream of 
the cleavage site, which would be the DNA interacting with the linker, affects the architecture of the active site/C-terminal domain. Even though the X-ray structure of the mutation-induced alterations in Top $1 \mathrm{p}$ is not currently available, these studies suggest that the conserved $\mathrm{Gly}^{721}$ residue acts as a flexible hinge within the active site of Top1 that enable linker domain flexibility and the structural alterations that accompany drug binding of the covalent enzyme-DNA complex.

As the Gly residue is conserved in human Top1 (Gly ${ }^{717}$ in hTop1), our next step was to determine if this residue has the same characteristics as in yTop1. When Gly ${ }^{717}$ in hTop1 was mutated to the negatively charged Asp there was no increase in CPT sensitivity when this mutant was expressed in yeast cells. This contrasts with the CPT hypersensitivity of yeast cells expressing yTop $1 \mathrm{G}^{721} \mathrm{D}$. However, important to note is that expressing wild-type hTop1 in yeast cells increases cell sensitivity to CPT one hundred fold compared to cells expressing yTop1. We considered that an upper threshold of CPTinduced DNA damage may have already been attained by CPT treatment of hTop1expressing yeast cells such that expression of $\mathrm{hTop} 1 \mathrm{G}^{717} \mathrm{D}$ had no effect. However, purified $h T o p 1 \mathrm{G}^{717} \mathrm{D}$ recapitulated the increase in enzyme sensitivity to CPT and also the alteration in tryptic digests seen with the yeast mutant enzyme, suggesting that mutation of this conserved residue induces a similar mechanism of increased CPT sensitivity in both yeast and human Top1, namely an structural alteration that drives CPT binding.

Using aforementioned suicide substrates, we observed that $h T o p 1 \mathrm{G}^{717} \mathrm{D}$ exhibited a dramatic increase in the rate of cleavage, regardless of the length of the DNA 3' to the site of scission. This mutant is distinct from the self-poisoning mutant $y$ Top $1 \mathrm{~N}^{726} \mathrm{H}$, which also displays an increased rate of DNA cleavage, in that $h T o p 1 \mathrm{G}^{717} \mathrm{D}$ did not 
produce elevated levels of covalent complex formation in the absence of CPT and in vivo toxicity in the absence of the drug. In cleavage reactions with the suicide substrates, increased cleavage at a second site was also observed, while in the steady state DNA cleavage assays no alteration in sequence specificity was detected. Based on these results, it might be that the increase in DNA cleavage is specific for the suicide substrates. As CPT does increase stabililization of the covalent, a second possibility might be that the increase rate of DNA cleavage by $\mathrm{G}^{717} \mathrm{D}$ coupled with the decreased rate of DNA religation induced by CPT binding enhance CPT poisining of $h T o p 1 \mathrm{G}^{717} \mathrm{D}$.

\subsection{THE DISTINCT LINKER DOMAINS PLAY A ROLE IN REGULATING CPT SENSITIVITY AND ENZYME CATALYSIS IN VIVO}

The differences between yeast and human Top1 Gly mutants in enhancing cell sensitivity to CPT and the altered DNA cleavage rates of $h T 0 p 1 \mathrm{G}^{717} \mathrm{D}$ prompted us to probe the role of the distinct linker domains in regulating CPT sensitivity and enzyme catalysis in vivo. This was accomplished by generating chimeric proteins where the various domains were exchanged using homologous recombination in yeast. The core and C-terminal domains of Top 1 are the most conserved regions of the enzyme, while the Nterminus and the linker are the most divergent in both amino acid sequence and size. Human Top1 has an N-terminus consisting of approximately 200 amino acids, while the smaller linker domain comprises only 45 amino acids. In yeast Top1, the relative length of the shorter N-terminal domain is only 140 amino acids long, while the yeast linker is 
twice the lenght of the human linker domain. However, overall the combined sizes of the N-terminal and linker domains are conserved.

Most of the protein-protein interactions involving Top1 have been mapped to the $\mathrm{N}$-terminal domain. For our experiments we first wanted to investigate what role these protein interactions might play in regulating cell sensitivity to CPT and therefore exchanged the N-termini between the two enzymes. No difference in CPT was observed when these N-terminal chimeric enzymes were expressed in yeast cells, refuting the simple notion that protein-protein interactions dictated by the $\mathrm{N}$-terminus impacts CPT sensitivity.

Next, we exchanged the linker domains between the human and yeast Top1 proteins. The chimeric yeast enzyme, which contained the much smaller human linker, exhibited decreased specific enzyme activity and a concomitant decrease in CPT sensitivity in yeast cells. Interestingly, the chimeric human enzyme containing the much larger yeast linker was cytotoxic in the absence of any drug. No alteration in specific activity, DNA affinity or covalent complex formation in the presence of absence to CPT could be detected in vitro, indicating that the toxicity might not be due to alterations in enzyme catalysis. To determine if toxicity was independent of Top1 catalyzed DNA cleavage, the active site Tyr was substituted with Phe, rendering the enzyme inactive. This catalytically inactive chimera was still toxic when overexpressed in yeast cells, indicating that cytotoxicity was not a consequence of covalent Top1-DNA adduct formation. These results lead us to posit that the lethal phenotype of cells expressing this inactive (hN, yL)-hTop $1 \mathrm{Y}^{723} \mathrm{~F}$ mutant protein resulted from alterations in protein interactions directed by the linker domain. 
Indeed, the expression of $\mathrm{hTop} 1 \mathrm{G}^{717} \mathrm{D}$ did not increase cell sensitivity to CPT, while expression of the corresponding yeast mutant $\left(y T o p 1 G^{721} \mathrm{D}\right)$ did. Yet, both mutant proteins demonstrated increased CPT sensitivity in steady state DNA cleavage assays. Taken together, these findings suggest that differences in the protein interactions between the yeast and human enzymes in yeast may be directed by the linker domain.

As the N-terminus is not present in any of the Top1 crystal structures solved to date, the position of this domain relative to the rest of the protein in unknown. However there has been suggestion that this domain interacts with the DNA downstream of the cleavage site (37). This region of the DNA has also been shown to interact with the linker domain (12). To determine if the $\mathrm{N}$-terminus has any affect on the cytotoxicity induced by the lethal chimera, the human $\mathrm{N}$-terminus was replaced with that of the yeast $\mathrm{N}$ terminus to yield a protein that contained the human core and C-terminus and the yeast linker and N-terminus. Surprisingly, cells expressing this "double" chimera were viable and sensitive to the same level of CPT as cells expressing wild-type hTop1. Moreover, the in vitro CPT sensitivity and sequence specificity of the (yN,yL)-hTop1 chimeric protein was comparable to that exhibited by wild-type hTop1, demonstrating that the CPT sensitivity of hTop1 is dictated by the core and C-terminal domains. The combination of the linker and the N-terminal domain, on the other hand, appear to regulate the in vivo activity of the enzyme, possibly in directing the formation of protein complexes that do nor occur are inappropriately regulated in the absence of the correct Nterminus/Linker combination.

To determine what role the linker might play in the increased sensitivity of the Gly mutant, the residue was mutated to Asp in the yeast Top1 chimera containing the 
human linker ((yN,hL)-yTop1). In comparison with wild-type yTop1, expression of (yN, hL)-yTop1 rendered cells resistant to CPT. However, mutating the Gly residue to Asp in (yN, hL)-yTop $1 \mathrm{pG}^{721} \mathrm{D}$ restored CPT sensitivity albeit not to the level of yTop $1 \mathrm{G}^{721} \mathrm{Dp}$. These experiments demonstrate that the $\mathrm{G}^{721} \mathrm{D}$ mutation increases CPT sensitivity independent of which linker domain is present, human or yeast. The human linker in the context of the yeast Top1 clamp did decrease the specific activity of the chimeric enzyme, although it does not appear to be due to a DNA binding defect, as the salt optimum for specific activity was not altered as compared to wild-type yTop1. As the linker domain places the active site Tyr/C-terminal domain in the correct position within the catalytic pocket, the shorter human linker might not position the yeast active Tyr/Cterminal domain in its optimal location for catalytic activity. The decrease in enzyme activity does not appear to be due to protein misfolding as it is stably expressed. One way to address the question would be to use suicide substrates to determine if this chimeric protein exhibits a defect in DNA cleavage and therefore a decrease in specific activity. As the Gly to Asp substitution increases Top1 sensitivity to CPT independent linker composition, the question then becomes if a linker domain is necessary at all to cause this increase. To answer this question, a linkerless yTop1 protein can be generated and then used to substitute the Gly with Asp. If this mutant enzyme still exhibits increased CPT sensitivity then the structural change induced by the mutation increases the binding of $\mathrm{CPT}$, while alterations in linker flexibility may play an indirect role in mediating interactions between the drug stabilized Top1-DNA intermediates and other protein complexes. 
To further test the hypothesis that there are differences in protein interactions dictated by the linker domain, we performed a two-hybrid screen to identify linker domain binding partners. The hTop1 linker and C-terminal domain fragment has previously been shown to fold correctly such that an active enzyme could be reconstituted with a separate polypeptide encoding the hTop1 core domain (39). For this screen, the corresponding yeast linker and C-terminal domain was used to avoid heterologous protein interactions. Using this polypeptide as bait, we identified 16 proteins that induced the expression of reporter genes. Three of these proteins have been previously shown to either physically or genetically interact with Top1 giving validity to the results. The three previously identified were Ubc9, Pol30 (PCNA) and Tif4631 (136, 191, 192), although no biological function have been demonstrated for these interactions. At this time, four of these proteins have been tested and confirmed as true interactors. Pol30 (PCNA), Nis1 and Ubc9 have been shown to interact with both the yeast and human Top1 linker domains, while Mrm1 only interacts with yTop1 linker. Mrm1 is a ribose methyltransferase and is essential for the formation of functional mitochondrial ribosomes. Mrm1 is located in the mitochondria, while Top1 is a nuclear protein. Although possibility therefore exist that this is not a true interaction due to their separate location, it is of interest to note that a human mitochondrial Top1 encoded by a different gene than its nuclear counterpart exist. This enzyme also contains a linker, but lacks the N-terminal domain. A longstanding question had been whether a Type 1B enzyme is required for mitochondrial DNA replication and transcription in yeast. This result might help elucidate this problem. 
Nis1 is located in the bud neck at the G2/M phase of the cell cycle, but has also been found in the nucleus. It is believed to be involved in mitotic sigaling, but not much more is know about this protein. As Top1 is known to play a role in maintaining chromosome condensation, this interaction might be of significance during mitosis.

The interaction of yTopl linker and C-terminal fragment with Pol30 (PCNA) is an intriguing result as this protein functions at multiple levels in directing DNA metabolic pathways. It has been shown to promote localization of replication factors with a consensus PCNA-binding domain, although the Top1 fragment used for this study did not contain this binding motif. When bound to DNA, Pol30 organizes various proteins involved in DNA replication and repair. It forms a homotrimer that clamps around duplex DNA and slides spontaneously along the bases. This sliding clamp moves along with the replication fork and functions as a scaffold for the recruitment of various proteins such as Pole and Pold, both involved in processive DNA replication (198). Many of the protein interactions with Pol30 are directed by post translation modification of this sliding clamp. For example sumoylated PCNA has been shown to recruit Srs1 and thereby prevent recombination during S-phase (199). Human Top1 has already been shown to bind to PCNA by affinity chromatography (192), but the binding site has not been mapped to a specific region or domain of Top1. As the C-terminal domain of Top1 is buried within the active site pocket created by the core, it is therefore very likely that Pol30 interacts with the linker region that extends away from the body of the enzyme. To map the exact Pol30 binding site on Top1, smaller fragments of Top1 can be tested for interaction. Top1 acts as a swivel to relieve torsional strain in the DNA created by the movement of the replication fork and it is possible that Pol30 will recruit Top1 to regions of high 
torsional strain. A second possible role for this interaction might be that the linker acts as a sensor for approaching replication forks to trigger the dissociation of Top1 from the DNA, thereby preventing DNA damage caused by the collision of the replication machinery and Top1 covalently bound to the DNA. The linker acting as a sensor might also function in sensing the increase in torsional strain caused by the oncoming replication forks which might increase catalytic activity or dissociation of the enzyme from the DNA. As Pol30 (PCNA) undergoes extensive post-translational modification to regulate replication and repair, it will be of interest to determine if Top1 binding is driven by a specific modification of Pol30.

The region of Top1 (both yeast and human) that was used as bait, namely the linker and C-terminal domain contains consensus SUMO sites (one in the yeast and two in the human fragment) and it is therefore not surprising to find that the second enzyme, Ubc9, in the sumoylation cascade can interact with this region of Top1. This also raises the question of whether sumoylation of the linker affects the binding with the other proteins, for example the interaction with Pol30. Ubc9 can by itself or with the help of an E3 ligase transfer SUMO (Smt3 in yeast) to Lys residues of target proteins, in this case Top1. Top1 has been shown to be sumoylated and initially the modification was believed to affect CPT sensitivity. Jacquiau et al. (136) however, showed that mutating the SUMO sites on Top1 did not affect CPT sensitivity. Interestingly, Siz2, an E3 ligase in the sumoylation cascade was also identified in this screen. The Top1-Siz2 interaction might be directed by Ubc9, such that Siz2 interacts with Ubc9 and not directly with Top1. Another interesting finding was that there seemed to be two Ubc9 populations in the cells expressing the Ubc9 fusion protein. The molecular weight of the smaller polupation 
corresponded to the Gal4 activation domain (AD)-Ubc9 fusion protein, while the other may be sumoylated Gal4 AD-Ubc9. Ubc9 is known to be sumoylated on Lys ${ }^{153}$ (194), thus it is possible that Top1 only interacts with sumoylated Ubc9. As all of these interactions were identified using fusion proteins, they will have to be confirmed by using full length wild-type Top1 (human and yeast) and Ubc9. To determine if the second Ubc9 population is truly sumoylated Ubc9, a mutant $\mathrm{Ubc} 9$, where Lys ${ }^{153}$ is mutated to an Ala or Arg, can be generated and tested to see if this population is still present. This mutant can also be used to determine if the interaction between Top1 and Ubc9 requires a sumoylated Ubc9. To determine if Top1 linker sumoylation is required for its interaction with other protein, the linker SUMO site can be mutated and this mutant tested for interactions with its binding partners.

Even though the results of the two-hybrid screen were not conclusive, the results are intriguing and it suggests specific paths that will be explored to expand our understanding of Top1 interactions and what role they mighty play in the function and regulation of this enzyme. It will be of great interest to know if the Top1 interaction with Pol30 in dependant/independent on the presence of DNA, if the two protein colocalize and if this colocalization changes during the cell cycle or with the treatment of CPT. Using ChIP-Chip analysis we might be able determine if the if the colocalization is a genome wide phenomena which might help us understand if the interaction is part of a repair pathway or if Top1 is simply recruited as a component of the replication machinery to replication despots.

In conclusion, the work presented here revealed that a single mutation in Top1 can increase the intrinsic CPT sensitivity of the enzyme. The mechanistic basis of this 
increased sensitivity appears to be a structural alteration of the active site Tyr/ C-terminal domain that enhances CPT binding to the covalent Top1-DNA intermediate. This result is of importance in this era of rational drug design to generate new compounds that might mimic/induce this structural change, thereby increasing the efficiency of CPT.

We further demonstrate that not only the N-terminus, but also the linker domain function as a docking site for interacting proteins, such as Pol30 (PCNA) and Ubc9, and knowledge of the biological function of these interactions will lead to a better understanding of the regulation of Top1 function and also pathways affecting the lesions induced by Top1 targeting drugs. 


\section{LIST OF REFERENCES}

1. Watson, J. D., and Crick, F. H. (1953) Nature 171(4361), 964-967.

2. Wang, J. C. (1996) Ann. Rev. Biochem. 65, 635-692.

3. Champoux, J. J. (2001) Ann. Rev. Biochem. 70, 369-413.

4. Wang, J. C. (2002) Nat. Rev. Mol. Cell. Biol. 3(6), 430-440.

5. Villa, H., Otero Marcos, A. R., Reguera, R. M., Balana-Fouce, R., Garcia-Estrada, C., Perez-Pertejo, Y., Tekwani, B. L., Myler, P. J., Stuart, K. D., Bjornsti, M. A., and Ordonez, D. (2003) J. Biol. Chem. 278(6), 3521-3526.

6. Hsiang, Y.-H., Hertzberg, R., Hecht, S., and Liu, L. F. (1985) J. Biol. Chem. 260, 14873-14878.

7. Corbett, K. D., and Berger, J. M. (2004) Annu. Rev. Biophys. Biomol. Struct. 33, 95-118.

8. Berger, J. M., Gamblin, S. J., Harrison, S. C., and Wang, J. C. (1996) Nature 379, 225-232.

9. Lima, C. D., Wang, J. C., and Mondragon, A. (1994) Nature 367, 138-146.

10. Champoux, J. J. (2002) Proc. Natl. Acad. Sci. U S A 99(19), 11998-12000.

11. Dekker, N. H., Rybenkov, V. V., Duguet, M., Crisona, N. J., Cozzarelli, N. R., Bensimon, D., and Croquette, V. (2002) Proc. Natl. Acad. Sci. U S A 99(19), 12126-12131.

12. Stewart, L., Redinbo, M. R., Qiu, X., Hol, W. G. J., and Champoux, J. J. (1998) Science 279, 1534-1541.

13. Koster, D. A., Croquette, V., Dekker, C., Shuman, S., and Dekker, N. H. (2005) Nature 434(7033), 671-674.

14. Eng, W.-K., Pandit, S. D., and Sternglanz, R. (1989) J. Biol. Chem. 264(23), 13373-13376.

15. Lynn, R. M., Bjornsti, M.-A., Caron, P. R., and Wang, J. C. (1989) Proc. Natl. Acad. Sci. U S A 86(10), 3559-3563.

16. Krogh, B. O., and Shuman, S. (2000) Molecular cell 5(6), 1035-1041.

17. Krogh, B. O., and Shuman, S. (2002) J. Biol. Chem. 277(8), 5711-5714.

18. Patel, A., Shuman, S., and Mondragon, A. (2006) J. Biol. Chem. 281(9), 60306037.

19. Shuman, S. (1998) BBA 1400(1-3), 321-337.

20. Leppard, J. B., and Champoux, J. J. (2005) Chromosoma 114(2), 75-85.

21. Brino, L., Urzhumtsev, A., Mousli, M., Bronner, C., Mitschler, A., Oudet, P., and Moras, D. (2000) J. Biol. Chem. 275(13), 9468-9475.

22. Morham, S. G., Kluckman, K. D., Voulomanos, N., and Smithies, O. (1996) Mol. Cell. Biol. 16, 6804-6809.

23. Zhang, C. X., Chen, A. D., Gettel, N. J., and Hsieh, T. S. (2000) Dev. Biol. 222(1), 27-40.

24. Lee, M. P., Brown, S. D., Chen, A., and Hsieh, T.-S. (1993) Proc. Natl. Acad. Sci. US A 90, 6656-6660. 
25. Christman, M. F., Dietrich, F. S., Levin, N. A., Sadoff, B. U., and G.R. Fink, G. R. (1993) Proc. Natl. Acad. Sci. U S A 90, 7637-7641.

26. Reid, R. J. D., Benedetti, P., and Bjornsti, M.-A. (1998) $B B A$ 1400, 289-300

27. Redinbo, M. R., Stewart, L., Kuhn, P., Champoux, J. J., and Hol, W. G. J. (1998) Science 279, 1504-1513.

28. Stewart, L., Ireton, G. C., and Champoux, J. J. (1996) J. Biol. Chem. 271, 76027608.

29. D'Arpa, P., Machlin, P. S., Ratrie, H., Rothfield, N. F., Cleveland, D. W., and Earnshaw, W. C. (1988) Proc. Natl. Acad. Sci. U S A 85, 2543-2547.

30. Stewart, L., Ireton, G. C., Parker, L. H., Madden, K. R., and Champoux, J. J. (1996) J. Biol. Chem. 271, 7593-7601.

31. Bjornsti, M.-A., and Wang, J. C. (1987) Proc. Natl. Acad. Sci. U S A 84, 89718975.

32. Albor, A., Kaku, S., and Kulesz-Martin, M. (1998) Cancer Res. 58, 2091-2094.

33. Bharti, A. K., Olson, M. O., Kufe, D. W., and Rubin, E. H. (1996) J. Biol. Chem. 271, 1993-1997.

34. Haluska, P. J., and Rubin, E. H. (1998) Adv. Enzyme Regul. 38, 253-262.

35. Pommier, Y., Pourquier, P., Fan, Y., and Strumberg, D. (1998) BBA 1400, 83106.

36. Shykind, B. M., Kim, J., Stewart, L., Champoux, J. J., and Sharp, P. A. (1997) Genes \& development 11, 397-407.

37. Lisby, M., Olesen, J. R., Skouboe, C., Krogh, B. O., Straub, T., Boege, F., Velmurugan, S., Martensen, P. M., Andersen, A. H., Jayaram, M., Westergaard, O., and Knudsen, B. R. (2001) J. Biol. Chem. 29, 29.

38. Redinbo, M. R., Champoux, J. J., and Hol, W. G. (2000) Biochemistry 39(23), 6832-6840.

39. Stewart, L., Ireton, G., and Champoux, J. (1997) J. Mol. Biol. 269, 355-372.

40. Christiansen, K., Bonven, B. J., and Westergaard, O. (1987) J. Mol. Biol. 193, 517-525.

41. Woo, M. H., Losasso, C., Guo, H., Pattarello, L., Benedetti, P., and Bjornsti, M. A. (2003) Proc. Natl. Acad. Sci. U S A 100(24), 13767-13772.

42. Redinbo, M. R., Champoux, J. J., and Hol, W. G. (1999) Curr. Opin. Sturct. Biol. 9, 29-36.

43. Christiansen, K., Svejstrup, A. B. D., Andersen, A. H., and Westergaard, O. (1993) J. Biol. Chem. 268(13), 9690-9701.

44. Svestrup, J. Q., Christiansen, K., Anderson, A. H., Lund, K., and Westergaard, O. (1990) J. Biol. Chem. 265, 12529-12535.

45. Stevnsner, T., Mortensen, U. H., Westergaard, O., and Bonven, B. J. (1989) J. Biol. Chem. 264(17), 10110-10113.

46. Been, M. D., and Champoux, J. J. (1984) J. Mol. Biol. 180(3), 515-531.

47. Bonven, B. J., Gocke, E., and Westergaard, O. (1985) Cell 41(2), 541-551.

48. Tanizawa, A., Bertrand, R., Kohlhagen, G., Tabuchi, A., Jenkins, J., and Pommier, Y. (1993) J. Biol. Chem. 268, 25463-25468.

49. Shen, C. C., and Shen, C.-K. (1990) J. Mol. Biol. 212, 67-78.

50. Krogh, S., Mortensen, U. H., Westergaard, O., and Bonven, B. J. (1991) Nucleic acids research 19(6), 1235-1241. 
51. Camilloni, G., Di Martino, E., Di Mauro, E., and Caserta, M. (1989) Proc. Natl. Acad. Sci. U S A 86(9), 3080-3084.

52. Madden, K. R., Stewart, L., and Champoux, J. J. (1995) EMBO Journal 14, 53995409.

53. Caserta, M., Camilloni, G., Venditti, S., Venditti, P., and Di Mauro, E. (1994) J. Cell. Biochem. 55, 93-97.

54. Zechiedrich, E. L., and Osheroff, N. (1990) EMBO Journal 9, 4555-4562.

55. Nitiss, J., and Wang, J. C. (1988) Proc. Natl. Acad. Sci. U S A 85, 7501-7505.

56. Raymond, M., Gros, P., Whiteway, M., and Thomas, D. Y. (1992) Science 256(5054), 232-234.

57. Bjornsti, M.-A., Benedetti, P., Viglianti, G. A., and Wang, J. C. (1989) Cancer Res. 49(22), 6318-6323.

58. Hertzberg, R. P., Caranfa, M. J., and Hecht, S. M. (1989) Biochemistry 28, 46294638.

59. Staker, B. L., Hjerrild, K., Feese, M. D., Behnke, C. A., Burgin, A. B., Jr., and Stewart, L. (2002) Proc. Natl. Acad. Sci. U S A 99(24), 15387-15392.

60. Redinbo, M. R., Stewart, L., Champoux, J. J., and Hol, W. G. (1999) J. Mol. Biol. 292(3), 685-696.

61. Stewart, L., Ireton, G. C., and Champoux, J. J. (1999) J. Biol. Chem. 274(46), 32950-32960.

62. Fiorani, P., Bruselles, A., Falconi, M., Chillemi, G., Desideri, A., and Benedetti, P. (2003) J. Biol. Chem. 278(44), 43268-43275.

63. Gellert, M. (1981) Annu. Rev. Biochem. 50, 879-910.

64. Yang, L., Rowe, T. C., and Liu, L. F. (1985) Cancer Res. 45(11 Pt 2), 5872-5876.

65. Burden, D. A., and Osheroff, N. (1998) BBA 1400(1-3), 139-154.

66. Eng, W.-K., Faucette, L., Johnson, R. K., and Sternglanz, R. (1988) Mol. Pharmacol. 34, 755-760.

67. Hsiang, Y.-H., and Liu, L. F. (1988) Cancer Res. 48, 1722-1726.

68. Wall, M. E., and Wani, M. C. (1995) Cancer Res. 55, 753-760.

69. Andoh, T., Ishii, K., Suzuki, Y., Ikegami, Y., Kusunoki, Y., Takemoto, Y., and Okada, K. (1987) Proc. Natl. Acad. Sci. U S A 84(16), 5565-5569.

70. Kjeldsen, E., Mollerup, S., Thomsen, B., Bonven, B. J., Bolund, L., and Westergaard, O. (1988) J. Mol. Biol. 202(2), 333-342.

71. Pommier, Y., Pourquier, P., Urasaki, Y., Wu, J., and Laco, G. S. (1999) Drug Resist. Updat. 2(5), 307-318.

72. Garcia-Carbonero, R., and Supko, J. G. (2002) Clin. Cancer Res. 8(3), 641-661.

73. Rodriguez-Galindo, C., Radomski, K., Stewart, C. F., Furman, W., Santana, V. M., and Houghton, P. J. (2000) Med. Pediatr. Oncol. 35(4), 385-402.

74. Rasheed, Z. A., and Rubin, E. H. (2003) Oncogene 22(47), 7296-7304.

75. Porter, S. E., and Champoux, J. J. (1989) Nucleic Acids Res. 17(21), 8521-8532.

76. Pommier, Y., Kohlhagen, G., Laco, G. S., Kroth, H., Sayer, J. M., and Jerina, D. M. (2002) J. Biol. Chem. 277(16), 13666-13672.

77. Pourquier, P., Waltman, J. L., Urasaki, Y., Loktionova, N. A., Pegg, A. E., Nitiss, J. L., and Pommier, Y. (2001) Cancer Res. 61(1), 53-58.

78. Sokhansanj, B. A., and Wilson, D. M., 3rd. (2004) Free Radic. Biol. Med. 37(3), 422-427. 
79. D'Arpa, P., Beardmore, C., and Liu, L. F. (1990) Cancer Res. 50(21), 6919-6924.

80. Nitiss, J., and Wang, J. (1996) Mol. Pharm. 50, 1095-1102.

81. Nitiss, J. L., and Wang, J.C. (1991) Yeast as a genetic system in the dissection of the mechanism of cell killing by topoisomerase-targeting anticancer drugs. In: Potmesil, M., and Kohn, K,W. (ed). DNA topoisomerases in cancer, Oxford University Press.

82. Pommier, Y., Tanizawa, A., and Kohn, K. W. (1994) Adv. Pharmacol. 29B, 73-92

83. Avemann, K., Knippers, R., Koller, T., and Sogo, J. M. (1988) Mol. Cell. Biol. 8, 3026-3034.

84. Li, T. K., and Liu, L. F. (2001) Annu. Rev. Pharmacol. Toxicol. 41, 53-77.

85. Chrencik, J. E., Staker, B. L., Burgin, A. B., Pourquier, P., Pommier, Y., Stewart, L., and Redinbo, M. R. (2004) J. Mol. Biol. 339(4), 773-784.

86. Goldwasser, F., Bae, I., Valenti, M., Torres, K., and Pommier, Y. (1995) Cancer Res. 55(10), 2116-2121.

87. Rothenberg, M. L., Eckardt, J. R., Kuhn, J. G., Burris, H. A., 3rd, Nelson, J., Hilsenbeck, S. G., Rodriguez, G. I., Thurman, A. M., Smith, L. S., Eckhardt, S. G., Weiss, G. R., Elfring, G. L., Rinaldi, D. A., Schaaf, L. J., and Von Hoff, D. D. (1996) J. Clin. Oncol. 14(4), 1128-1135.

88. Santana, V. M., Zamboni, W. C., Kirstein, M. N., Tan, M., Liu, T., Gajjar, A., Houghton, P. J., and Stewart, C. F. (2003) Clin. Cancer. Res. 9(2), 633-640.

89. Gupta, E., Luo, F., Lallo, A., Ramanathan, S., Vyas, V., Rubin, E., and Sinko, P. (2000) Anticancer Res. 20(2A), 1013-1016.

90. Schellens, J. H., Maliepaard, M., Scheper, R. J., Scheffer, G. L., Jonker, J. W., Smit, J. W., Beijnen, J. H., and Schinkel, A. H. (2000) Annals of the New York Academy of Sciences 922, 188-194.

91. Sparreboom, A., Loos, W. J., Burger, H., Sissung, T. M., Verweij, J., Figg, W. D., Nooter, K., and Gelderblom, H. (2005) Cancer biology \& therapy 4(6), 650-658

92. Reid, R. J., Kauh, E. A., and Bjornsti, M. A. (1997) J. Biol. Chem. 272(18), 12091-12099.

93. Danks, M. K., Garrett, K. E., Marion, R. C., and Whipple, D. O. (1996) Cancer Res. 56, 1664-1673.

94. Tsurutani, J., Nitta, T., Hirashima, T., Komiya, T., Uejima, H., Tada, H., Syunichi, N., Tohda, A., Fukuoka, M., and Nakagawa, K. (2002) Lung Cancer 35(3), 299-304.

95. Knab, A. M., Fertala, J., and Bjornsti, M.-A. (1993) J. Biol. Chem. 268(30), 22322-22330.

96. Hann, C. L., Carlberg, A. L., and Bjornsti, M.-A. (1998) J. Biol. Chem. 273, 31519-31527.

97. Eng, W. K., McCabe, F. L., Tan, K. B., Mattern, M. R., Hofmann, G. A., Woessner, R. D., Hertzberg, R. P., and Johnson, R. K. (1990) Mol. Pharmacol. 38(4), 471-480.

98. Gupta, R. S., Gupta, R., Eng, B., Lock, R. B., Ross, W. E., Hertzberg, R. P., Caranfa, M. J., and Johnson, R. K. (1988) Cancer Res. 48(22), 6404-6410.

99. Fertala, J., Vance, J. R., Pourquier, P., Pommier, Y., and Bjornsti, M. A. (2000) J. Biol. Chem. 275(20), 15246-15253. 
100. Edwards, T. K., Saleem, A., Shaman, J. A., Dennis, T., Gerigk, C., Oliveros, E., Gartenberg, M. R., and Rubin, E. H. (2000) J. Biol. Chem. 275(46), 36181-36188.

101. Pourquier, P., Ueng, L.-M., Fertala, J., Wang, D., Park, H.-J., Essigman, J. M., Bjornsti, M.-A., and Pommier, Y. (1999) J. Biol. Chem. 274, 8516-8523.

102. Pourquier, P., Ueng, L.-M., Kolhagen, G., Mazumder, A., Gupta, M., Kohn, K. W., and Pommier, Y. (1997) J. Biol. Chem. 272, 7792-7796.

103. Colley, W. C., van der Merwe, M., Vance, J. R., Burgin, A. B., Jr., and Bjornsti, M. A. (2004) J. Biol. Chem. 279(52), 54069-54078.

104. Koster, D. A., Palle, K., Bot, E. S., Bjornsti, M. A., and Dekker, N. H. (2007) Nature 448(7150), 213-217.

105. Pommier, Y., Redon, C., Rao, V. A., Seiler, J. A., Sordet, O., Takemura, H., Antony, S., Meng, L., Liao, Z., Kohlhagen, G., Zhang, H., and Kohn, K. W. (2003) Mutat. Res. 532(1-2), 173-203.

106. Furuta, T., Takemura, H., Liao, Z. Y., Aune, G. J., Redon, C., Sedelnikova, O. A., Pilch, D. R., Rogakou, E. P., Celeste, A., Chen, H. T., Nussenzweig, A., Aladjem, M. I., Bonner, W. M., and Pommier, Y. (2003) J. Biol.Chem. 278(22), 2030320312.

107. Adachi, N., Ikeda, H., and Kikuchi, A. (1994) Nucleic acids research 22(20), 4229-4233.

108. Arnaudeau, C., Tenorio Miranda, E., Jenssen, D., and Helleday, T. (2000) Mutat. Res. 461(3), 221-228.

109. Haber, J. E. (2000) Mutat. Res. 451(1-2), 53-69.

110. Cliby, W. A., Lewis, K. A., Lilly, K. K., and Kaufmann, S. H. (2002) J. Biol. Chem. 277(2), 1599-1606.

111. Wan, S., Capasso, H., and Walworth, N. C. (1999) Yeast 15(10A), 821-828.

112. Fiorani, P., Reid, R. J., Schepis, A., Jacquiau, H. R., Guo, H., Thimmaiah, P., Benedetti, P., and Bjornsti, M. A. (2004) J. Biol. Chem. 279(20), 21271-21281.

113. Megonigal, M. D., Fertala, J., and Bjornsti, M. A. (1997) J. Biol. Chem. 272(19), 12801-12808.

114. Pouliot, J. J., Yao, K. C., Robertson, C. A., and Nash, H. A. (1999) Science 286(5439), 552-555.

115. Pommier, Y., Barcelo, J. M., Rao, V. A., Sordet, O., Jobson, A. G., Thibaut, L., Miao, Z. H., Seiler, J. A., Zhang, H., Marchand, C., Agama, K., Nitiss, J. L., and Redon, C. (2006) Progress in nucleic acid research and molecular biology 81, 179-229.

116. Liu, C., Pouliot, J. J., and Nash, H. A. (2002) Proc. Natl. Acad. Sci. U S A 99(23), 14970-14975.

117. Vance, J. R., and Wilson, T. E. (2002) Proc. Natl. Acad. Sci. U S A 99(21), 13669-13674.

118. Bastin-Shanower, S. A., Fricke, W. M., Mullen, J. R., and Brill, S. J. (2003) Mol. Cell. Biol. 23(10), 3487-3496.

119. Doe, C. L., Ahn, J. S., Dixon, J., and Whitby, M. C. (2002) J. Biol. Chem. 277(36), 32753-32759.

120. Reid, R. J., Fiorani, P., Sugawara, M., and Bjornsti, M. A. (1999) Proc. Natl. Acad. Sci. US A 96(20), 11440-11445. 
121. Baker, S. D., Wadkins, R. M., Stewart, C. F., Beck, W. T., and Danks, M. K. (1995) Cytometry 19, 134-145.

122. Christensen, M. O., Krokowski, R. M., Barthelmes, H. U., Hock, R., Boege, F., and Mielke, C. (2004) J. Biol. Chem. 279(21), 21873-21882.

123. Nakielny, S., and Dreyfuss, G. (1999) Cell 99(7), 677-690.

124. Muller, M. T., Pfund, W. P., Mehta, V. B., and Trask, D. K. (1985) EMBO Journal 4(5), 1237-1243.

125. Seither, P., Iben, S., and Grummt, I. (1998) J. Mol. Biol. 275(1), 43-53.

126. Cheutin, T., O'Donohue, M. F., Beorchia, A., Vandelaer, M., Kaplan, H., Defever, B., Ploton, D., and Thiry, M. (2002) J. Cell Sci. 115(Pt 16), 3297-3307.

127. Christensen, M. O., Barthelmes, H. U., Boege, F., and Mielke, C. (2002) J. Biol. Chem. 277(39), 35932-35938.

128. Rose, K. M., Szopa, J., Han, F. S., Cheng, Y. C., Richter, A., and Scheer, U. (1988) Chromosoma 96(6), 411-416.

129. Christman, M. F., Dietrich, F. S., and Fink, G. R. (1988) Cell 55, 413-425.

130. Shaiu, W. L., and Hsieh, T. S. (1998) Mol. Cell. Biol. 18(7), 4358-4367.

131. Buckwalter, C. A., Lin, A. H., Tanizawa, A., Pommier, Y. G., Cheng, Y. C., and Kaufman, S. H. (1996) Cancer Res. 56(7), 1674-1681.

132. Christensen, M. O., Barthelmes, H. U., Feineis, S., Knudsen, B. R., Andersen, A. H., Boege, F., and Mielke, C. (2002) J. Biol. Chem. 277(18), 15661-15665.

133. Mao, Y., Mehl, I. R., and Muller, M. T. (2002) Proc. Natl. Acad. Sci. U S A 99(3), 1235-1240.

134. Mo, Y. Y., Yu, Y., Shen, Z., and Beck, W. T. (2002) J. Biol. Chem. 277(4), 29582964.

135. Rallabhandi, P., Hashimoto, K., Mo, Y. Y., Beck, W. T., Moitra, P. K., and D'Arpa, P. (2002) J. Biol. Chem. 277(42), 40020-40026.

136. Jacquiau, H. R., van Waardenburg, R. C., Reid, R. J., Woo, M. H., Guo, H., Johnson, E. S., and Bjornsti, M. A. (2005) J. Biol. Chem. 280(25), 23566-23575.

137. Kowalska-Loth, B., Girstun, A., Trzcinska, A. M., Piekielko-Witkowska, A., and Staron, K. (2005) Biochem. Biophys. Res. Commun. 331(2), 398-403.

138. Gilmour, D. S., Pflugfelder, G., Wang, J. C., and Lis, J. T. (1986) Cell 44(3), 401407.

139. Fleischmann, G., Pflugfelder, G., Steiner, E. K., Javaherian, K., Howard, G. C., Wang, J. C., and Elgin, S. C. (1984) Proc. Natl. Acad. Sci. U S A 81(22), 69586962.

140. Brill, S., DiNardo, S., Voelkel-Meiman, K., and Sternglanz, R. (1987) Nature 326, 414-416.

141. Durban, E., Goodenough, M., Mills, J., and Busch, H. (1985) EMBO Journal 4(11), 2921-2926.

142. Durban, E., Mills, J. S., Roll, D., and Busch, H. (1983) Biochem. Biophys. Res. Commun. 111(3), 897-905.

143. Hathaway, G. M., and Traugh, J. A. (1982) Curr. Top. Cell. Regul. 21, 101-127.

144. Kaiserman, H. B., Ingebritsen, T. S., and Benbow, R. M. (1988) Biochemistry 27(9), 3216-3222.

145. Pommier, Y., Kerrigan, D., Hartman, K. D., and Glazer, R. I. (1990) J. Biol. Chem. 265(16), 9418-9422. 
146. Tse-Dinh, Y. C., Wong, T. W., and Goldberg, A. R. (1984) Nature 312(5996), 785-786.

147. Darby, M. K., Schmitt, B., Jongstra-Bilen, J., and Vosberg, H. P. (1985) EMBO Journal 4(8), 2129-2134.

148. Ferro, A. M., and Olivera, B. M. (1984) J. Biol. Chem. 259(1), 547-554.

149. Kasid, U. N., Halligan, B., Liu, L. F., Dritschilo, A., and Smulson, M. (1989) J. Biol. Chem. 264(31), 18687-18692.

150. Smith, H. M., and Grosovsky, A. J. (1999) Carcinogenesis 20(8), 1439-1443.

151. Mao, Y., Desai, S. D., and Liu, L. F. (2000) J. Biol. Chem. 275(34), 26066-26073.

152. Bjornsti, M. A. (2002) Cancer Cell 2(4), 267-273.

153. Goffeau, A., Barrell, B. G., Bussey, H., Davis, R. W., Dujon, B., Feldmann, H., Galibert, F., Hoheisel, J. D., Jacq, C., Johnston, M., Louis, E. J., Mewes, H. W., Murakami, Y., Philippsen, P., Tettelin, H., and Oliver, S. G. (1996) Science 274(5287), 546, 563-547.

154. Bennett, C. B., Lewis, L. K., Karthikeyan, G., Lobachev, K. S., Jin, Y. H., Sterling, J. F., Snipe, J. R., and Resnick, M. A. (2001) Nature genetics 29(4), 426434.

155. Bovee, T. F., Helsdingen, R. J., Rietjens, I. M., Keijer, J., and Hoogenboom, R. L. (2004) The Journal of steroid biochemistry and molecular biology 91(3), 99-109.

156. Woo, M. H., Vance, J. R., Marcos, A. R., Bailly, C., and Bjornsti, M. A. (2002) J. Biol. Chem. 277(6), 3813-3822.

157. Fiorani, P., and Bjornsti, M. A. (2000) Annals of the New York Academy of Sciences 922(1), 65-75.

158. Champoux, J. J. (2000) Annals of the New York Academy of Sciences 922(1), 5664.

159. Redon, C., Pilch, D. R., Rogakou, E. P., Orr, A. H., Lowndes, N. F., and Bonner, W. M. (2003) EMBO Rep. 4(7), 678-684.

160. Simon, J. A., Szankasi, P., Nguyen, D. K., Ludlow, C., Dunstan, H. M., Roberts, C. J., Jensen, E. L., Hartwell, L. H., and Friend, S. H. (2000) Cancer Res. 60(2), 328-333.

161. Zhang, H., and Siede, W. (2003) Mutat. Res. 527(1-2), 37-48.

162. Pourquier, P., and Pommier, Y. (2001) Adv. Cancer Res. 80(45), 189-216.

163. Chrencik, J. E., Burgin, A. B., Pommier, Y., Stewart, L., and Redinbo, M. R. (2003) J. Biol. Chem. 278(14), 12461-12466.

164. Fiorani, P., Amatruda, J. F., Silvestri, A., Butler, R. H., Bjornsti, M. A., and Benedetti, P. (1999) Mol. Pharmacol. 56(6), 1105-1115.

165. Kauh, E. A., and Bjornsti, M. A. (1995) Proc. Natl. Acad. Sci. U S A 92(14), 6299-6303.

166. Kaiser, C., Michaelis, S., and Mitchell, A. (1994) Methods in Yeast Genetics, 1994 Edition Ed., Cold Spring Harbor Laboratory Press.

167. Lesher, D. T., Pommier, Y., Stewart, L., and Redinbo, M. R. (2002) Proc. Natl. Acad. Sci. U S A 99(19), 12102-12107.

168. Stivers, J. T., Shuman, S., and Mildvan, A. S. (1994) Biochemistry 33(1), 327339.

169. Levin, N. A., Bjornsti, M.-A., and Fink, G. R. (1993) Genetics 133(4), 799-814.

170. Pommier, Y. (2006) Nat. Rev. Cancer 6(10), 789-802. 
171. Pourquier, P., Pilon, A. A., Kolhhagen, G., Mzumder, A., Sharma, A., and Pommier, Y. (1997) J. Biol. Chem. 272, 26441-26447.

172. Koster, D. A., Palle, K., Bot, E. S., Bjornsti, M.-A., and Dekker, N. H. (2007) Nature (in press).

173. Chillemi, G., Fiorani, P., Benedetti, P., and Desideri, A. (2003) Nucleic Acids Res. 31(5), 1525-1535.

174. Losasso, C., Cretaio, E., Palle, K., Pattarello, L., Bjornsti, M. A., and Benedetti, P. (2007) J. Biol. Chem. 282(13). 9855-9864.

175. Staker, B. L., Feese, M. D., Cushman, M., Pommier, Y., Zembower, D., Stewart, L., and Burgin, A. B. (2005) J. Med. Chem. 48(7), 2336-2345.

176. Montaudon, D., Palle, K., Rivory, L. P., Robert, J., Douat-Casassus, C., Quideau, S., Bjornsti, M. A., and Pourquier, P. (2007) J. Biol. Chem. 282(19), 1440314412.

177. Fiorani, P., Chillemi, G., Losasso, C., Castelli, S., and Desideri, A. (2006) Nucleic Acids Res. 34(18), 5093-5100.

178. O'Neil, K. T., and DeGrado, W. F. (1990) Science 250(4981), 646-651.

179. Wang, J. C. (2002) Nat. Rev. Mol. Cell. Biol. 3(6), 430-440.

180. Koster, D. A., Palle, K., Bot, E. S., Bjornsti, M. A., and Dekker, N. H. (2007) Nature 448(7150), 213-217.

181. Hsiang, Y., Lihou, M. G., and Liu, L. F. (1989) Cancer Res. 49, 5077-5082.

182. Zhang, H., Barcelo, J. M., Lee, B., Kohlhagen, G., Zimonjic, D. B., Popescu, N. C., and Pommier, Y. (2001) Proc. Natl. Acad. Sci. U S A 98(19), 10608-10613.

183. Losasso, C., Cretaio, E., Palle, K., Pattarello, L., Bjornsti, M. A., and Benedetti, P. (2007) J. Biol. Chem. 282, 9855-9864.

184. van der Merwe, M., and Bjornsti, M. A. ((submitted)) J. Biol. Chem.

185. van Waardenburg, R. C., Duda, D. M., Lancaster, C. S., Schulman, B. A., and Bjornsti, M. A. (2006) Mol. Cell. Biol. 26(13), 4958-4969.

186. Burgin, A. B., Huizenga, B. N., and Nash, H. A. (1995) Nucleic Acids Res. 23, 2973-2979.

187. Horowitz, D. S., and Wang, J. C. (1987) J. Biol. Chem. 262, 5339-5344.

188. Elledge, S. J., Mulligan, J. T., Ramer, S. W., Spottswood, M., and Davis, R. W. (1991) Proc. Natl. Acad. Sci. U S A 88(5), 1731-1735.

189. Gyuris, J., Golemis, E., Chertkov, H., and Brent, R. (1993) Cell 75(4), 791-803.

190. Durfee, T., Becherer, K., Chen, P. L., Yeh, S. H., Yang, Y., Kilburn, A. E., Lee, W. H., and Elledge, S. J. (1993) Genes \& development 7(4), 555-569.

191. Krogan, N. J., Peng, W. T., Cagney, G., Robinson, M. D., Haw, R., Zhong, G., Guo, X., Zhang, X., Canadien, V., Richards, D. P., Beattie, B. K., Lalev, A., Zhang, W., Davierwala, A. P., Mnaimneh, S., Starostine, A., Tikuisis, A. P., Grigull, J., Datta, N., Bray, J. E., Hughes, T. R., Emili, A., and Greenblatt, J. F. (2004) Molecular cell 13(2), 225-239.

192. Loor, G., Zhang, S. J., Zhang, P., Toomey, N. L., and Lee, M. Y. (1997) Nucleic Acids Res. 25(24), 5041-5046.

193. Bossis, G., and Melchior, F. (2006) Molecular cell 21(3), 349-357.

194. Bencsath, K. P., Podgorski, M. S., Pagala, V. R., Slaughter, C. A., and Schulman, B. A. (2002) J. Biol. Chem. 277(49), 47938-47945.

195. Moldovan, G. L., Pfander, B., and Jentsch, S. (2007) Cell 129(4), 665-679. 
196. Liu, L. F., Desai, S. D., Li, T. K., Mao, Y., Sun, M., and Sim, S. P. (2000) Annals of the New York Academy of Sciences 922(1), 1-10.

197. Losasso, C., Cretaio, E., Palle, K., Pattarello, L., Bjornsti, M. A., and Benedetti, P. (2007) J. Biol. Chem. 282(13), 9855-9864.

198. Majka, J., and Burgers, P. M. (2004) Progress in nucleic acid research and molecular biology 78, 227-260.

199. Pfander, B., Moldovan, G. L., Sacher, M., Hoege, C., and Jentsch, S. (2005) Nature 436(7049), 428-433. 


\section{VITA}

Mariè van der Merwe was born in Welkom, South Africa on October 15, 1974. She graduated from Potchefstroom Gymnasium in December, 1992. In 1996 she received a Bachelor of Science and in 1998 a Bachelor of Science Honours in Biochemistry from the University of Potchefstroom. She enrolled in Interdisciplinary program at the University of Tennessee in 2002. 